\title{
How to move forward with evidence-based practice in nursing
}

Citation for published version (APA):

Friesen - Storms, JHHM. (2019). How to move forward with evidence-based practice in nursing: towards a personalised approach in cancer aftercare. [Doctoral Thesis, Maastricht University]. Maastricht University. https://doi.org/10.26481/dis.20190614jfs

Document status and date:

Published: 01/01/2019

DOI:

10.26481/dis.20190614jfs

Document Version:

Publisher's PDF, also known as Version of record

\section{Please check the document version of this publication:}

- A submitted manuscript is the version of the article upon submission and before peer-review. There can be important differences between the submitted version and the official published version of record.

People interested in the research are advised to contact the author for the final version of the publication, or visit the DOI to the publisher's website.

- The final author version and the galley proof are versions of the publication after peer review.

- The final published version features the final layout of the paper including the volume, issue and page numbers.

Link to publication

\footnotetext{
General rights rights.

- You may freely distribute the URL identifying the publication in the public portal. please follow below link for the End User Agreement:

www.umlib.nl/taverne-license

Take down policy

If you believe that this document breaches copyright please contact us at:

repository@maastrichtuniversity.nl

providing details and we will investigate your claim.
}

Copyright and moral rights for the publications made accessible in the public portal are retained by the authors and/or other copyright owners and it is a condition of accessing publications that users recognise and abide by the legal requirements associated with these

- Users may download and print one copy of any publication from the public portal for the purpose of private study or research.

- You may not further distribute the material or use it for any profit-making activity or commercial gain

If the publication is distributed under the terms of Article $25 \mathrm{fa}$ of the Dutch Copyright Act, indicated by the "Taverne" license above, 

How to move forward with evidence-based practice in nursing: towards a personalised approach in cancer aftercare 
(C) Jolanda Friesen-Storms, Maastricht 2019, All rights reserved

The research presented in this dissertation was conducted at:

Care and Public Health Research Institute (CAPHRI), Department of Family Medicine, Maastricht University, Maastricht, the Netherlands. CAPRHI participates in the Netherlands School of Primary Care Research (CaRe).

and

Research Centre for Autonomy and Participation for Persons with a Chronic Illness, Faculty of Health, Zuyd University of Applied Sciences, Nursing department, Heerlen, the Netherlands.

The research presented in this dissertation was supported by the Regional Attention and Action for Knowledge Circulation (SIA-RAAK) under programme number: 2009-8-10P, the Netherlands Organisation for Scientific Research (NWO) under project number: 023.002.031, Alpe d'HuZes (Dutch Cancer Society) under project number: KWF MAC 2014-7024, the Laurentius Hospital Roermond and the Zuyderland Medical Center.

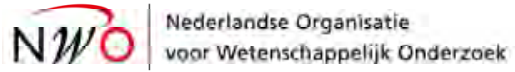

All rights are reserved. No part of this book may be reproduced or transmitted in any form or by any means, without the written permission of the author or, where appropriate, the publisher of the article.

Cover: Evelien Jagtman

Layout: Tiny Wouters-Lenssen

Printed by: Gildeprint Enschede

ISBN: 978-94-6323-537-2 
How to move forward with evidence-based practice in nursing:

towards a personalised approach in cancer aftercare

\section{PROEFSCHRIFT}

ter verkrijging van de graad van doctor aan de Universiteit Maastricht, op gezag van de Rector Magnificus, Prof. dr. Rianne M. Letschert, volgens het besluit van het College van Decanen, in het openbaar te verdedigen op vrijdag 14 juni 2019 om 14.00 uur

door

Jolanda Hubertina Hermina Maria Friesen-Storms 


\section{Promotores}

Prof. dr. A.J.H.M. Beurskens, Maastricht University en Zuyd Hogeschool Heerlen

Prof. dr. T. van der Weijden, Maastricht University

\section{Copromotores}

Dr. G. J.J.W. Bours, Zuyd Hogeschool, Heerlen en Maastricht University

Dr. K.S.G. Jie, Zuyd Hogeschool, Heerlen en Zuyderland Medisch Centrum SittardGeleen, Heerlen

\section{Beoordelingscommissie}

Prof. dr. S.M.G. Zwakhalen (voorzitter), Maastricht University

Prof. dr. M. van den Akker, Johann Wolfgang Goethe University, Frankfurt, Germany

Prof. dr. G.M.J. Bos, Maastricht University

Dr. P.J.M. Kil, Elisabeth-TweeSteden Ziekenhuis, Tilburg

Prof. dr. H. Vermeulen, Radboud University, Nijmegen 


\section{Contents}

Chapter 1 General introduction 7

Part I Implementation of evidence-based practice in nursing 25

$\begin{array}{lll}\text { Chapter } 2 & \text { Systematic implementation of evidence-based practice (EBP) in } \quad 27\end{array}$

a clinical nursing setting: a participatory action research project Journal of Clinical Nursing 2014;24:57-68

Chapter 3 Teaching and implementing evidence-based practice in a hospital unit with mainly secondary vocational trained nurses: Lessons Learned

The Journal of Continuing Education in Nursing 2017; 48(9):407-412

Part II Integration of shared decision making with evidence-based practice in chronic nursing care

Chapter 4 Shared decision making in chronic care in the context of evidence-based practice in nursing: a discussion International Journal of Nursing Studies 2015;52:393-402

Part III

A personalised approach in cancer aftercare

Chapter 5 A conversation approach based on shared goal-setting and shared decision making for nurses in cancer aftercare: a developmental study European Journal of Oncology Nursing 2018; 35: 107-116

Chapter 6 Perceived facilitating and restricting factors for healthcare professionals to adopting a patient decision aid for breast cancer aftercare: a cross-sectional study Under review: minor revisions. Patient Education and Counseling

Chapter 7 There is more to learn from stories of lymphoma survivors to further improve aftercare: a narrative inquiry

Submitted

Chapter $8 \quad$ General discussion

Summary

Samenvatting

Valorisation

Dankwoord

Curriculum vitae

List of publications 



\section{Chapter 1}

General introduction

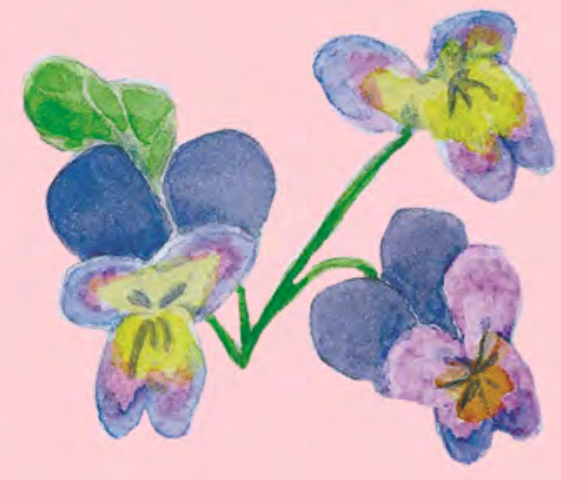


Chapter 1 


\section{General introduction}

Evidence-based practice integrates the best research evidence with clinical expertise and the patient's preferences and values in the decision-making process for individual patient care. ${ }^{1}$ Evidence-based practice is widely recognised as being relevant to the improvement of nursing care. ${ }^{2-4}$ The rationale for evidence-based practice is based on the claim that an intervention needs to be justified based on research evidence of the effectiveness of the intervention ${ }^{5}$ because this leads to a better standard of care. ${ }^{6}$

Shared decision making is a process that is meant to result in the health care professional and the patient jointly arriving at a health care choice that is based on the best available research evidence, clinical expertise, and the values of the informed patient. ${ }^{7,8}$ The rationale for shared decision making is based on research evidence that patients want to participate more in their own health care decisions ${ }^{9,10}$ and on the ethical principle of patient autonomy. ${ }^{5}$

Although shared decision making and evidence-based practice are two different concepts and processes, shared decision making acknowledges the importance of evidence-based practice, and evidence-based practice acknowledges the importance of integrating patient values and clinical expertise with research evidence. Furthermore, there is growing insight that evidence-based practice and shared-decision making should be integrated in the decision-making process. ${ }^{11}$

Suffering from a chronic condition affects a person's life. Chronically ill people encounter consequences like fatigue, insecurity, depression and restrictions in daily functioning. They are challenged to deal with these consequences and often have to adapt their lives to cope with these consequences. ${ }^{12,13}$ To support these patients in managing their condition in their daily lives, it is important to involve them in decision making and provide them with a personalised approach that addresses their individual experiences, preferences and values. ${ }^{14-17}$ In nursing, therefore, shared decision making seems particularly useful in chronic care. For many people cancer survival, regardless of their disease status, increasingly means living with a chronic condition ${ }^{18}$ for which a lot of decisions have to be made about evidence based aftercare. ${ }^{19,20}$ We defined evidence based aftercare as follows: aftercare includes interventions such as education, physical training and psychosocial support that helps patients to deal with potential issues of cancer and its treatment. ${ }^{21,22}$ The Dutch Health Council recommends improvements in aftercare. $^{21}$

Acknowledging the need for an integrated approach of evidence-based practice and shared decision making, the research presented in this thesis focuses on strategies to implement an integrated approach of evidence-based practice and shared decision 
making in chronic care in nursing, especially in cancer aftercare. The thesis is divided in three parts. In part one the focus is on implementation of evidence-based practice in nursing; in part two the focus is on the integration of shared decision making with evidence-based practice in chronic nursing care; and in part three the focus is on strategies to implement an integrated approach of evidence-based practice and shared decision making in cancer aftercare.

In this introduction the state of the art in evidence-based practice and shared decision making in nursing at the start of this research project, is reviewed. Additionally, the nursing context is described, and the need for and integrated approach of evidencebased practice and shared decision making in cancer aftercare in nursing is explained. The general introduction ends with the aim, the research questions and an outline of the thesis.

\section{Evidence-based practice in nursing}

Evidence based practice (EBP) has its origin in medical science (evidence-based medicine). Although there are several definitions of evidence-based medicine (EBM), the most common definition is: the integration of best research evidence with clinical expertise and the patient's preferences and values in making decisions about individual patient care. ${ }^{23}$ (See Figure 1.1).

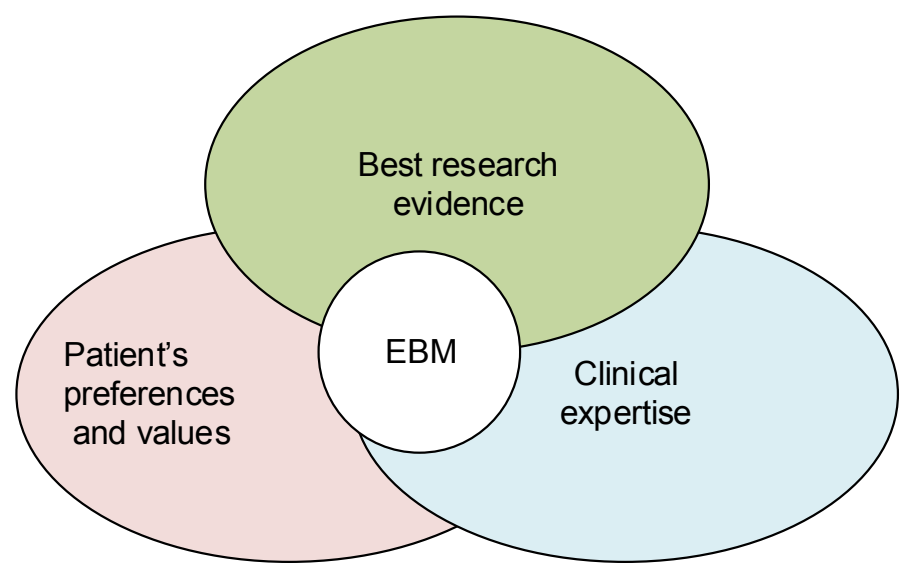

Figure 1.1 Evidence-based medicine ${ }^{23}$ 
Given the popularity of EBM, the phrase has been radically expanded, adopted and adapted by other disciplines within the health care arena. The nursing discipline adopted the term evidence-based nursing (EBN). ${ }^{24}$ However, EBP has become a generic term used across various health care professions, including nursing. EBP is key to delivering the highest quality of healthcare and ensuring the best patient outcomes. ${ }^{1,-2,25}$

Several EBP models have been reported, most of which share the following five process steps: (1) formulate questions, (2) efficiently search for the highest quality and most relevant research evidence, (3) appraise the research evidence critically, (4) integrate the research evidence, clinical expertise and the patient's values into the individual decision-making process, and (5) evaluate the process and result. ${ }^{26,27}$

The main focus in EBP is on the level of the quality of the underlying research evidence ${ }^{28-30}$, and less focus is on the individual patient's values and clinical expertise in the decision-making process about patient care. ${ }^{9,11,28}$ Different levels of quality are often represented in the form of a hierarchical pyramid, in which systematic reviews of randomised controlled trials and high-quality single randomised controlled trials are regarded and presented as forming the top, while editorials and expert opinions are making up the bottom of that hierarchy. ${ }^{31}$ Nevertheless, the level of research evidence on efficacy often cannot be directly applied in clinical practice. To facilitate the interpretation of research evidence into clinical practice, recently more and more evidence-based clinical practice guidelines have been developed by working groups of guideline developers. Furthermore, nurses and other health care professionals started with developing critically appraised topics, that are short summaries of evidence on topics of interest, usually focussed around a clinical question. ${ }^{32,33}$ These critically appraised topics may be helpful for nurses to implement research evidence into daily practice.

\section{Shared decision making in nursing}

Next to EBP the communication model Shared Decision Making (SDM) has gained interest in health care and nursing as the gold standard for integrating patient's values in the decision making about patient care. ${ }^{7,8,34} \mathrm{SDM}$ is said to be the crux of patientcentred care. ${ }^{7,8}$ SDM was first used in preference-sensitive decisions regarding medical screening, preventive, diagnostic, treatment and palliative options. In preferencesensitive decisions there is insufficient evidence about outcomes or there is a need to trade off known benefits or harms between competing options. ${ }^{35,36}$ Most research about SDM is related to physicians. ${ }^{8,37}$ However, nurses can have a substantial role in applying SDM in clinical practice. ${ }^{38}$ 
Various models have been developed to demonstrate how SDM can be applied in clinical practice, although a complete consensus on what SDM entails has not yet been reached. ${ }^{39}$ The essential elements of $\mathrm{SDM}^{39}$ are: define and explain the problem to the patient, present options to the patient, discuss the pros and cons with the patient, support patients to clarify their values and preferences, discuss the patient's ability and self-efficacy, discuss the health care professional's knowledge and recommendations, check and clarify the patient's understanding, make or explicitly defer the decision, and arrange follow up. Ideally, SDM would also include: provision of unbiased information, definition of the patient's desired role of involvement, presentation of evidence including probabilities of treatment outcomes, and mutual agreement. ${ }^{36,39}$

A well-known model of SDM specifically created by nurses is 'The Ottawa Decision Support Framework' ${ }^{40,41}$ The framework consist of three steps: assessing the patient's and practitioner's determinants of decisions to identify decision support needs, providing decision support tailored to the patient's needs, and evaluating the decisionmaking process and outcomes. 'The Ottawa Decision Support Framework' has been extensively validated through decisional needs assessment studies with patients, the public, and healthcare professionals. ${ }^{41}$ Elwyn et al. ${ }^{42}$ developed a relatively simple SDM model in light of applicability for clinical practice (See Figure 1.2).

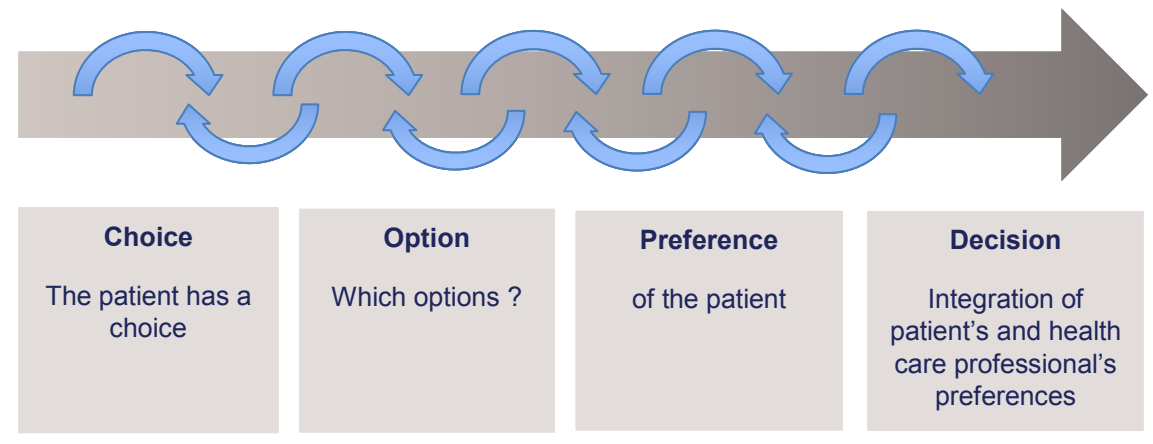

Figure 1.2 A model for shared decision making ${ }^{42}$

The essential elements of the model include choice talk, option talk, preference talk and decision talk. More specifically, the term 'choice talk' is used to refer to the act of clarifying that reasonable options are available to patients, option talk refers to providing detailed information about the pros and cons of each option, preferences talk refers to eliciting the patient's preferences, and decision talk refers to supporting the 
process of considering the patient's preferences (including the patient's right to opt out of making a decision) and deciding on the best option. ${ }^{42}$

To facilitate SDM in clinical practice, decision aids have been developed, evaluated, and implemented. Patient decision support tools or decision aids are decision support interventions that help people make choices by describing why and where choices exist and by providing information about the possible consequences of choices. ${ }^{43}$ Their format can be diverse, ranging from printed fact sheets and booklets to CD-ROMs, videos and interactive websites. ${ }^{44}$

When such decision aids are used, patients have been shown to have more knowledge about their health condition and experience less decisional conflict, while the amount of patients who are passive in the decision-making process as well as the amount of patients who remain unable to make decisions decreases, as compared to a situation in which the decision aids are not used. ${ }^{36}$

\section{Implementation of evidence-based practice and shared decision making in nursing}

In nursing, decisions are usually made on the basis of informal and experiential resources, instead of on scientific research and protocols. ${ }^{45-47}$

Implementation of EBP in nursing is challenging, and many barriers related to the implementation of EBP have been reported. ${ }^{4,48-50}$ And although implementation strategies have been developed to address these barriers, EBP is still insufficiently implemented in routine nursing practice. ${ }^{29,50-52}$

In nursing, factors that influence the successfulness of the implementation of EBP, include:

1. Internal factors: nurses' attitudes, perceptions, and their competencies in EBP. ${ }^{4}$

2. Social factors: local opinion leaders in nursing teams and the nursing culture, in which certain rituals, habits and routines usually prevail. ${ }^{53}$

3. Organisational factors: time for research activities, hospital size, staffing support, organisational innovativeness, integration of recommendations into organisational structures and processes, access to resources and research findings, the format and usability of clinical practice guidelines, organisational climate, provision of education, availability of knowledge and skills within organisations, interorganisational collaboration, finances, and workload; and

4. Societal factors such as crises in nursing or workforce shortages. ${ }^{48-50}$

To develop nurses' EBP competencies, various learning and teaching strategies exist. Several systematic reviews ${ }^{54,55}$ highlight teaching strategies concerning the different 
process steps of EBP, for example strategies that attempt to improve the formulation of answerable questions $^{54}$ (step 1 in EBP), and other strategies, like Journal Clubs, that attempt to improve critical appraisal skills (step 3 in EBP) $)^{55-57}$.

Khan $^{58}$ suggests that interactive and clinically integrated teaching and learning activities produce better results than standalone didactic teaching. One reason for this might be that, in interactive and clinically integrated teaching and learning activities, a real clinical problem is identified, evidence is sought actively, there is an emphasis on the resolution of clinical problems, knowledge and skills are used in practice, and knowledge and skills are learned while solving real clinical problems.

Implementation of EBP has so far concentrated on the process steps 1 (formulate questions), 2 (efficiently search research evidence), and 3 (appraise the research evidence critically). Step 4 (integrate the research evidence, clinical expertise, and the patient's values into the individual decision-making process) has received little attention. ${ }^{9}$

SDM is, like EBP, not well implemented in nursing practice. ${ }^{8,37}$ However, as stated in the previous section, most studies of SDM are related to SDM by physicians and there are only a few studies of implementation of SDM by nurses. These studies put forward that the following barriers seem to prevent the successful implementation of SDM in nursing: a lack of nurses' awareness, knowledge, and skills; inadequate training for nurses on how to practice SDM; a lack of time in clinical practice interfering with developing new skills; and unclear administrative direction about health professionals' roles in SDM. ${ }^{38,59}$

Although SDM and EBP are described and implemented as two different concepts and processes, they have similarities and it is acknowledged that SDM has the potential to strengthen the decision-making process (step 4) in EBP., ${ }^{9,11,60}$ However, how this can be accomplished is still unclear. ${ }^{9}$

\section{The context of nursing}

Nurses are often in the frontline of health care delivery. As a result, they play a critical role in identifying and responding to changes in patients' health status, preventing adverse events and supporting patients throughout their care journey. ${ }^{61}$

Nurses work across all sectors and settings of the health care system and are integral to the delivery of a range of health services, from community-based primary health care to inpatient care in the tertiary sector. Nursing is a diverse discipline which encompasses a range of overlapping roles, including: delivering clinical care, coordinating patient care, 
coordinating safety and quality improvements, and research and education. As a profession, nursing takes a holistic, patient-centred approach to care. Nurses assess and monitor changes in patients' health status, develop care plans, deliver clinical nursing interventions, support patients' self-care and evaluate patient outcomes. Nurses often work in multidisciplinary teams in which they may have a role as patient advocates and alliance builders: nurses coordinate and motivate care teams, families and patients to support patient wellbeing. ${ }^{61}$

Nurses are educated on various educational levels and with various specialisations. In the Netherlands we have three nursing degrees: 1. a master's degree, the nurse practitioner (in Dutch: verpleegkundig specialist); 2. a bachelor's degree; 3.; a registered nurse with secondary vocational training of four years, which is lower than a bachelor's degree. In the Netherlands, registered nurses with a secondary vocational training are trained to work in less complex care situations according to protocols, whereas nurses who have completed a bachelor are trained for more complex care situations and are trained in critical clinical reasoning for situations where existing protocols and EBP guidelines are not sufficient or have to be integrated. The nurse practitioner is trained in critical clinical reasoning to deliver complex evidence-based specialised nursing care and medical care according to protocol within a specific field of expertise. ${ }^{62}$

Although educational levels and training of nurses in the Netherlands differ, in daily clinical nursing practice and in existing law, the bachelor and secondary vocational degree, are not clearly differentiated regarding direct patient care. In the Netherlands, the nursing professions and their future competencies are more clearly defined at this moment for implementation in the next years. ${ }^{62}$

\section{The need for an integrated approach of evidence-based practice and shared decision making for nurses in cancer aftercare}

Evidence-based aftercare supports patients to deal with potential issues of cancer and its treatment. ${ }^{21,22}$ Due to improved cancer survival rates and rising global cancer incidence rates, an increasing number of cancer survivors will live with the consequences of cancer and its treatment. ${ }^{63}$

Cancer and its treatment are often associated with long-term physical and psychosocial issues such as fatigue, pain, reduced muscle mass and strength, problems in daily functioning, fear, anxiety, sleep disturbances, reduced self-esteem and problems returning to work. ${ }^{20,64}$ As such, cancer survivors often face the imposing task of 
rebuilding their physical and psychological health, which requires evidence-based aftercare. $^{21,64}$

However, few cancer survivors actually receive any comprehensive aftercare. Acknowledging this discrepancy between what cancer survivors need and what they are currently being offered, patient advocacy groups, expert consensus panels, and governmental reports have recommended making improvements in the quality of cancer aftercare. ${ }^{21,64}$ To improve the quality of cancer aftercare evidence-based guidelines have been developed to deal with a number of potential issues and needs that cancer survivors might experience. ${ }^{20,65-67}$ These guidelines recommend a systematic approach for aftercare based on shared decision-making, in which problems, goals, and wishes are systematically screened, shared, and integrated in a cancer survivorship plan. ${ }^{20,66,67}$ These guidelines have not been systematically implemented yet.

Registered oncology nurses can significantly contribute to the quality of aftercare. ${ }^{68}$ Generally speaking, oncology nurses focus more on teaching and control of potential issues, whereas physicians concentrate more on medical diagnostics and therapy. Nurses also focus on personal goals and needs and are often more available than physicians to patients and families who call with problems associated with symptom management. 68

Although the relevance of an integrated approach of EBP and SDM directed at patient goals in cancer aftercare in nursing is acknowledged, there is no systematic approach developed for daily nursing practice. ${ }^{20}$

\section{Aim and research questions}

As already stated in the first part of this general introduction, the research presented in this thesis focuses on strategies to implement an integrated approach of EBP and SDM in chronic care in nursing, especially in cancer aftercare. To maximise learning about how to merge these two concepts, we first studied the implementation of EBP in nursing in order to gain optimum insight and understanding of this topic. In the second part, we discuss the relevance of involving patients in applying EBP to individual decision making and subsequently introduce the integration of EBP and SDM in chronic care in nursing. In the third part of the thesis we focus on strategies to implement an integrated approach of EBP and SDM in cancer aftercare in nursing.

The aim of the research reported in this thesis is to gain knowledge about implementation of EBP in nursing, to find a way to integrate SDM with EBP in a chronic care environment in nursing, and to develop a strategy for an integrated approach of EBP and SDM in daily nursing practice in the individual aftercare for cancer survivors. 
The underlying research paradigm of our research project is social constructivism.

Social constructivism refers to the idea that multiple interpretations of reality (can) coexist, and posits that there is value in gaining a deeper understanding of how individuals construct reality within their natural context. ${ }^{69-70}$

For each part of the thesis a research question is formulated:

1. What is the feasibility of implementing evidence-based practice in daily nursing care by interactive and clinically integrated methods?

2. How can shared decision making be integrated with evidence-based practice in chronic nursing care?

3. Which strategy, tailored to the needs of patients and proxies, nurses and other health care professionals, seems worthwhile and feasible to implement an integrated approach of evidence-based practice and shared decision making in cancer aftercare?

This research project consisted of six different studies, as outlined in Figure 1.3. In the first and second study EBP was implemented in nursing, and the results of which were used to answer the first research question. In the third study a literature study was conducted and presented in a discussion on the integration of EBP and SDM in chronic care to answer the second research question. The findings of the implementation studies and the discussion paper were used in the fourth study in an iterative user centred method for developing complex interventions to develop and pre-test a conversation approach integrating EBP and SDM in cancer aftercare in nursing. Based on the results of the pre-test of the conversation approach some tools of the approach were digitized into an, improved, second version of a patient decision aid (PtDA) for breast cancer aftercare. The first version of the PtDA was developed by Klaassen et al. ${ }^{71}$. In the fifth study, an adoption study was conducted to study the main factors influencing the adoption of the PtDA. In the sixth study, a narrative inquiry was conducted to gain indepth insight into the personal stories of lymphoma survivors in early aftercare who have been exposed to our conversation approach that integrates EBP and SDM. The results of the fourth, fifth and sixth study were used to answer the third research question. 


\section{Research question}

1. What is the feasibility of implementing EBP in daily nursing practice by interactive and clinically integrated methods?

2. How can shared decision making be nursing care? integrated with evidence-based practice in

Method

Result
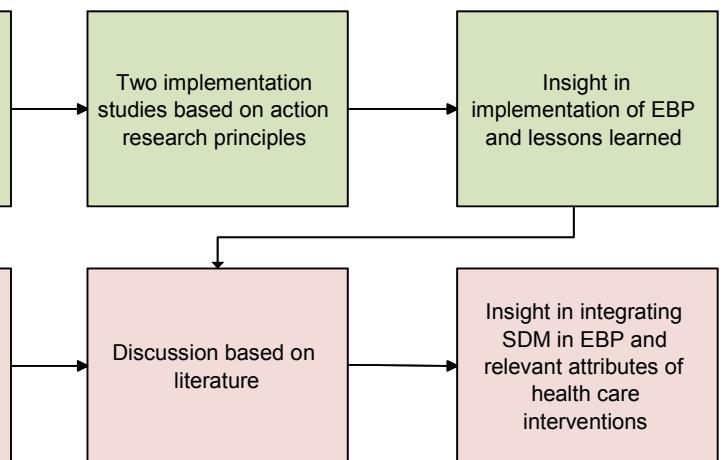

3. Which strategy, tailored to the needs of patients and proxies, nurses and other health care professionals seems worthwhile and feasible to implement EBP in clinical and shared decision making in cancer aftercare?

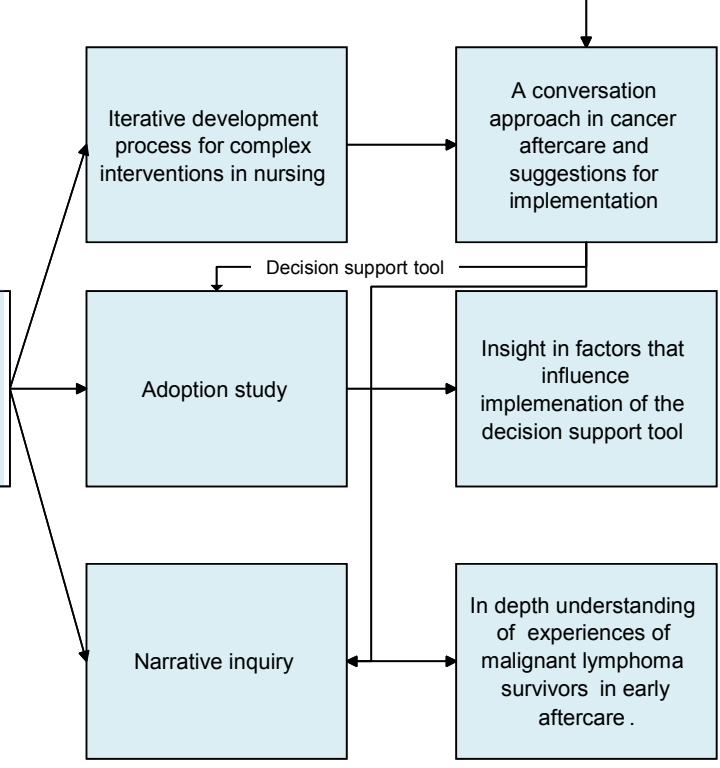

Figure 1.3 The systematic approach of the entire research project 


\section{Thesis outline}

Chapter 2 presents a participatory action research study to implement evidence-based practice in nursing on a pulmonary unit of a rural hospital in the South of the Netherlands with secondary vocational trained nurses. The working group used the development and implementation of a unit-specific EBP protocol for nursing discharge planning as a vehicle to implement EBP.

Chapter 3 presents a study in which EBP was systematically implemented in nursing using a systematic implementation model ${ }^{72}$ with elements of action research. The setting in which this study was conducted was a large teaching hospital in the South of the Netherlands with some nurses with a bachelor degree and other nurses with secondary vocational training. The working group used a patient case discussion meetings based on EBP principles ${ }^{73}$ as a vehicle to implement EBP in daily practice.

Chapter 4 presents a discussion on the relevance of SDM in chronic care and on the way(s) in which it can be integrated with EBP in nursing.

Chapter 5 describes the development and pre-test of a conversation approach integrating EBP and SDM directed at patient goals for nurses in cancer aftercare. In the conversation approach several tools were embedded.

Chapter 6 presents an adoption study of the perceived facilitators and barriers for health care professionals to adopt a PtDA for breast cancer aftercare that integrates some tools that were embedded in our developed conversation approach.

Chapter 7 presents a narrative inquiry to study in-depth experiences of lymphoma survivors in early aftercare that have been exposed to our conversation approach.

Chapter 8 discusses the main findings of the research project, gives an insight into both methodological as well as theoretical considerations, and the implications of the results for nursing practice, education, and research. 


\section{References}

1. Melnyk BM, Fineout-Overholt E. Evidence-Based Practice in Nursing \& Healthcare. Wolters Kluwer Lippincott Williams \& Wilkins: Philadelphia, Baltimore, New York, London, Buenos Aires, Hong Kong, Sydney, Tokyo: 2014.

2. Grimshaw J, Eccles M, Thomas R, MacLennan G, Ramsay C, Fraser C, et al. Toward Evidence-Based Quality Improvement: Evidence (and its Limitations) of the Effectiviness of Guideline Dissemination and Implementation Strategies 1966-1998. J Gen Intern Med. 2006;21:S14-20.

3. Heater BS, Becker AM, Olson RK. Nursing Interventions and Patient Outcomes: A Meta-Analysis of Studies. Nursing Research. 1988;37(5):303-7.

4. Melnyk BM, Fineout-Overholt E. The Evidence-Based Practice Beliefs and Implementation Scales: Psychometric Properties of Two New Instrument. Worldviews Evid Based Nurs. 2008:208-16.

5. Parker M. The ethics of evidence-based patient choice. Health Expect. 2001;4(2):87-91.

6. Armstrong D. Clinical autonomy, individual and collective: the problem of changing doctors' behaviour. Soc Sci Med. 2002;55(10):1771-7.

7. Charles C, Gafni A, Whelan T. Shared decision-making in the medical encounter: What does it mean? (or it takes at least two to tango). Soc Sci Med. 1997;44(5):681-92.

8. Légaré F, Ratté S, Stacey D, Kryworuchko J, Gravel K, Graham I, et al. Interventions for improving the adoption of shared decision making by healthcare professionals. Cochrane Database Syst Rev. 2010(5): 1-45.

9. Barratt A. Evidence based medicine and shared decision making: the challenge of getting both evidence and preferences into health care. Patient Educ Couns. 2008;73:407-12.

10. Coulter A. Paternalism or partnership? Patients have grown up-and there's no going back. BMJ. 1999;319(7212):719-20.

11. Satterfield JM, Spring B, Brownson RC, Mullen EJ, Newhouse RP, Walker BB, et al. Toward a transdisciplinary model of evidence-based practice. Milbank Q. 2009;87(2):368-90.

12. Lawn S, Schoo A. Supporting self-management of chronic health conditions: common approaches. Patient Educ Couns. 2010;80(2):205-11.

13. World Health Organization 2008-20013: action plan for the global strategy for the prevention and control of non-communicable diseases. World Health Organization, 2009. Retrieved from: http://apps.who.int/iris/bitstream/handle/10665/44009/9789241597418_eng.pdf?sequence=1

14. Clark NM, Nelson BW, Valerio MA, Gong ZM, Taylor-Fishwick JC, Fletcher M. Consideration of Shared Decision Making in Nursing: A Review of Clinicians' Perceptions and Interventions. The Open Nursing Journal. 2009;3:65-75.

15. Zoffmann V, Harder I, Kirkevold M. A Person-Centered Communication and Reflection Model: Sharing Decision-Making in Chronic Care. Qual Health Res. 2008;18(5):670-85.

16. Coulter A, Entwistle VA, Eccles A, Ryan S, Shepperd S, Perera R. Personalised care planning for adults with chronic or long-term health conditions. Cochrane Database Syst Rev. 2015(3):Cd010523.

17. Bodenheimer T, Lorig K, Holman H, Grumbach K. Patient self-management of chronic disease in primary care. JAMA. 2002;288(19):2469-75.

18. Phillips JL, Currow DC. Cancer as a chronic disease. 2010;17(2):47-50.

19. Cancer research Uk. 2012. Retrieved from: http://www.cancerresearchuk.org/health-professional/ cancer-statistics/survival\#heading-Zero

20. Integraal Kankercentrum Nederland [Comprehensive Cancer Centre the Netherlands ] (IKNL). 2011. Richtlijn Herstel na Kanker [Guideline Cancer survivorship care]. Retrieved from: http://www.oncoline.nl/herstel-na-kanker.

21. The Health Council of the Netherlands. Follow-up in oncology. Identify objectives, substantiate actions. The Hague: Health Council of the Netherlands; 2007. Retrieved from: https://www.gezondheidsraad.nl/en/task-and-procedure/areas-of-activity/optimum-healthcare/followup-in-oncology-identify-objectives. 
22. Hewitt M, Greenfield S, Stovall EL. 2005. From Cancer Patient to Cancer Survivors: Lost in Transition. Institute of Medicine, Washington DC.

23. Sackett D, Richardson W, Rosenberg WH. Evidence-based medicine: how to practice an teach EBM. 2nd ed. Edinburgh: Churchill Livingstone; 2000. 1-12 p.

24. French P. The development of EBN. J Adv Nurs. 1999;29:72-8.

25. Arnold FW, LaJoie A, Brock GN, et al. Improving outcomes in elderly patients with community-acquired pneumonia by adhering to national guidelines: Community-acquired pneumonia organization international cohort study results. Arch Intern Med. 2009;169(16):1515-24.

26. Sackett DL, Rosenberg WMC. The need for evidence-based medicine. J R Soc Med. 1995;88(11):620-4.

27. Gawlinski A, Rutledge D. Selecting a Model for Evidence-Based Practice Changes: A Practical Approach. AACN Adv Crit Care. 2008;19(3):291-300.

28. Melnyk BM, Fineout-Overholt E. Consumer preferences and values as an integral key to evidence-based practice. Nurs Adm Q. 2006;30(2):123-7.

29. Squires JE, Hutchinson AM, Bostrom A, O'Rourke HM, Cobban SJ, Estabrooks CA. To what extent do nurses use research in clinical practice ? A systematic review. Implement Sci. 2011;6(21):1-43.

30. Squires J, Estabrooks C, Gustavsson P, Wallin L. Individual determinants of research utilization by nurses: a systematic review update. Implement Sci. 2011;6(1):1.

31. Mantzoukas S. A review of evidence-based practice, nursing research and reflection: levelling the hierarchy. J Clin Nurs. 2008;17(2):214-23.

32. de Brouwer CPM, Kannt IJ, Smits LJM, Voogd AC. Training Critical Appraisel of a Topic Maastricht: Mediview BV; 2009.

33. Maaskant J, Storm-Versloot M, Vermeulen H. CATs boekje deel 1. Amsterdam: UMC; 2013.

34. Weston WW. Informed and shared decision-making: the crux of patient-centered care. Cmaj. 2001;165(4):438-9.

35. Elwyn G, Edwards A, Kinnersley P, Grol R. Shared decision making and the concept of equipoise: the competences of involving patients in healthcare choices. Br J Gen Pract. 2000;50(460):892-9.

36. Stacey D, Legare F, Col NF, Bennett CL, Barry MJ, Eden KB, et al. Decision aids for people facing health treatment or screening decisions. Cochrane Database Syst Rev. 2014(1):Cd001431.

37. Legare F, Ratte S, Gravel K, Graham ID. Barriers and facilitators to implementing shared decision-making in clinical practice: update of a systematic review of health professionals' perceptions. Patient Educ Couns. 2008;73(3):526-35.

38. Stacey D, Murray MA, Légaré F, Dunn S, Menard P. Decision Coaching to Support Shared Decision Making: A Framework, Evidence, and Implications for Nursing Practice, Education, and Policy. Worldviews Evid Based Nurs. 2008;5(1):25-35.

39. Makoul G, Clayman ML. An integrative model of shared decision making in medical encounters. Patient Educ Couns. 2006;60(3):301-12.

40. O'Connor A, Stacey D, Jacobsen MJ. Ottowa Decision Support Tutorial 2007 Retrieved from: https://decisionaid.ohri.ca/ODST/pdfs/ODST.pdf.

41. Ottowa Decision Support Framework: Update, Gaps and Research Priorities 2010 Retrieved from: http://decisionaid.ohri.ca/docs/ODSF-workshop/ODSF-Workshop-Summary.pdf.

42. Elwyn G, Frosch D, Thomson R, Joseph-Williams N, Lloyd A, Kinnersley P, et al. Shared Decision Making: A Model for Clinical Practice. J Gen Intern Med. 2012;27(10):1361-7.

43. van der Weijden T, Boivin A, Burgers J, Schünemann HJ, Elwyn G. Clinical practice guidelines and patient decision aids. An inevitable relationship. J Clin Epidemiol. 2012;65(6):584-9.

44. Elwyn G, Frosch D, Volandes AE, Edwards A, Montori VM. Investing in deliberation: a definition and classification of decision support interventions for people facing difficult health decisions. Med Decis Making. 2010;30(6):701-11.

45. Spenceley SM, O'Leary KA, Chizawsky LL, Ross AJ, Estabrooks CA. Sources of information used by nurses to inform practice: An integrative review. Int J Nurs Stud. 2008;45(6):954-70.

46. Rycroft-Malone J, Fontenla M, Seers K, Bick D. Protocol-based care: the standardisation of decisionmaking? J Clin Nurs. 2009;18(10):1490-500.

47. Traynor M, Boland M, Buus N. Autonomy, evidence and intuition: nurses and decision-making. J Adv Nurs. 2010;66(7):1584-91. 
48. Achterberg v, T., Schoonhoven L, Grol R. Nursing implementation Science: how evidence-based nursing requires evidence based implementation. J Nurs Scholarsh. 2008;40(4):302-10.

49. Larrabee JH, Janney MA, Ostrow CL, Withrow ML, Hobbs GRJ, Burant C. Predicting registered nurse job satisfaction and intent to leave. Journal of Nursing Administration. 2003;33:271-83.

50. Thompson D, Estabrooks C, Scott-Findlay S, Moore K, Wallin L. Interventions aimed at increasing research use in nursing: a systematic review. Implement Sci. 2007;2:15.

51. Schuster M, McGlynn E, Brook R. How good is the quality of health care in the United States ? Milbank Q. 1998;76:517-63.

52. Grol R. Successes and failures in the implementation of evidence-based guidelines for clinical practice. Med care. 2001;39(8 (Suppl 2)):II 46-54.

53. Zeitz K, McCutcheon $\mathrm{H}$. Tradition, rituals and standards, in a realm of evidenced based nursing care. Contemp Nurse. 2005;18(3):300-8.

54. Horsley T, O'Neill J, McGowan J, Perrier L, Kane G, Campbell C. Interventions to improve question formulation in professional practice and self-directed learning. Cochrane Database Syst Rev. 2010(5): $1-31$.

55. Horsley T, Hyde C, Santesso N, Parkes J, Milne R, Stewart R. Teaching critical appraisal skills in healthcare settings. Cochrane Database Syst Rev. 2011;9(11):1-35.

56. Currier RL, Schneider MR, Heubi JE. Taking Journal Clubs off Autopilot: A case study of teaching literature evaluation skills to preclinical MD/PhD Students. Med Sci Educ. 2013; 23(4): 572-77.

57. Lachance C. Nursing journal clubs: A literature review on the effective teaching strategy for continuing education and evidence-based practice. J Contin Educ Nurs. 2014;45(12):559-65.

58. Khan KS, Coomarasamy A. A hierarchy of effective teaching and learning to acquire competence in evidenced-based medicine. BMC Med Educ. 2006;6:59.

59. Stacey D, Graham ID, O'Connor AM, Pomey MP. Barriers and facilitators influencing call center nurses' decision support for callers facing values-sensitive decisions: a mixed methods study. Worldviews Evid Based Nurs. 2005;2(4):184-95.

60. Vandvik PO, Brandt L, Alonso-Coello P, Treweek S, Akl EA, Kristiansen A, et al. Creating clinical practice guidelines we can trust, use, and share: a new era is imminent. Chest. 2013;144(2):381-9.

61. James KM. Incorporating complexity science theory into nursing curricula. Creative nursing. 2010;16(3): 137-42.

62. Verpleegkundigen en verzorgenden 2020[Nurses and carers]. Beroepsprofiel verpleegkundige [Professional profile nurses]. Utrecht: V\&V20202; 2012.

63. Internation Agency for research on cancer. GLOBOCAN. 2012 Availeble at: http://gco.iarc.fr/

64. Hewitt M, Greenfield S, Stovall EL. From Cancer Patient to Cancer Survivors: Lost in Transition. Washington DC: Institute of Medicine; 2005.

65. Kent EE, Arora NK, Rowland JH, Bellizzi KM, Forsythe LP, Hamilton AS, et al. Health information needs and health-related quality of life in a diverse population of long-term cancer survivors. Patient Educ Couns. 2012;89(2):345-52.

66. Integraal Kankercentrum Nederland [Comprehensive Cancer Centre the Netherlands] (IKNL). 2011. Richtlijn Oncologische revalidatie [Guideline Oncological Rehabilitation]. Retrieved from: http://www.oncoline.nl/oncologische-revalidatie.

67. Integraal Kankercentrum Nederland [Comprehensive Cancer Centre the Netherlands] (IKNL), 2010. Richtlijn: detecteren behoefte psychosociale zorg [Guideline: Detecting the need for psychosocial care]. Retrieved from: http://www.oncoline.nl/detecteren-behoefte-psychosociale-zorg.

68. Schulman LN. Efficient and Effective Models for Integrating Advanced Practice Professionals into Oncology Practice. ASCO EDUCATIONAL BOOK: PROFESSIONAL DEVELOPMENT Optimizing the Role of Advanced Practice Professionals in Oncology Practice: American Association of Clinical Oncology; 2013 : e377-e9.

69. Flick U. An introduction to qualitative research Los Angelos, London, New Delhi, Singapore, Washington DC Sage 2014.

70. Polit DF, Beck CT. Nursing research: generating and assessing evidence for nursing practice. Philadelphia (PA): Williams \& Wilkins; 2014. 
71. Klaassen L, Dirksen C, Boersma L, Hoving C. Developing an aftercare decision aid; assessing health professionals' and patients' preferences. Eur J Cancer Care (Engl). 2017.

72. Grol R, Wensing M, Eccles M, Davis D. Improving Patient Care: The Implementation of Change in Health Care. West Sussex: John Wiley \& Sons, Ltd; 2013.

73. Vermeulen $\mathrm{H}$, Ingrid van Tellingen I, Jolanda Maaskant J, Simons R. Verpleegkundige dossierbespreking: een goed idee voor implementatie van EBP? [The patient case discussion for nurses: A good idea for implementation of EBP?] Nederlands Tijdschrift voor Evidence Based Practice. 2009;7(4):4-7. 




\section{Chapter}

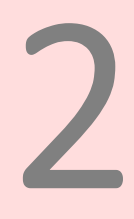

Systematic implementation of evidence-based practice (EBP) in a clinical nursing setting: a participatory action research project

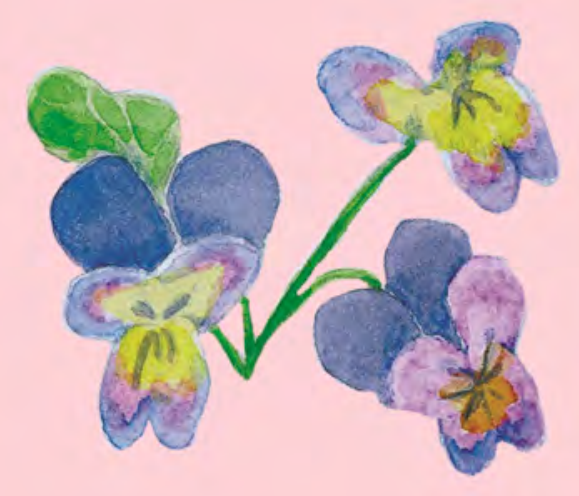

Jolanda H.H.M. Friesen-Storms Albine Moser Sandra van der Loo Anna J.H.M. Beurskens Gerrie J.J.W. Bours Journal of Clinical Nursing 2014;24:57-68 


\section{Abstract}

Aims and objectives

To describe the process of implementing evidence-based practice (EBP) in a clinical nursing setting.

\section{Background}

EBP has become a major issue in nursing, it is insufficiently integrated in daily practice and its implementation is complex.

\section{Design}

Participatory action research.

\section{Method}

The main participants were nurses working in a lung unit of a rural hospital. A multi-method process of data collection was used during the observing, reflecting, planning and acting phases. Data were continuously gathered during a 24-month period from 2010 to 2012, and analysed using an interpretive constant comparative approach. Patients were consulted to incorporate their perspective.

\section{Results}

A best-practice mode of working was prevalent on the ward. The main barriers to the implementation of EBP were that nurses had little knowledge of EBP and a rather negative attitude towards it, and that their English reading proficiency was poor. The main facilitators were that nurses wanted to deliver high-quality care and were enthusiastic and open to innovation. Implementation strategies included a tailored interactive outreach training and the development and implementation of an evidence-based discharge protocol. The academic model of EBP was adapted. Nurses worked according to the EBP discharge protocol but barely recorded their activities. Nurses favourably evaluated the participatory action research process.

\section{Conclusions}

Action research provides an opportunity to empower nurses and to tailor EBP to the practice context. Applying and implementing EBP is difficult for front-line nurses with limited EBP competencies.

\section{Relevance to clinical practice}

Adaptation of the academic model of EBP to a more pragmatic approach seems necessary to introduce EBP into clinical practice. The use of scientific evidence can be facilitated by using preappraised evidence. For clinical practice, it seems relevant to integrate scientific evidence with clinical expertise and patient values in nurses' clinical decision making at the individual patient level. 


\section{Introduction}

The relevance of evidence-based practice (EBP) is widely recognized in health care today. ${ }^{1}$ International and national social and political developments ${ }^{2-4}$ force nurses to base their practice on evidence. EBP has been defined as the conscientious, explicit and judicious use of current best evidence in making decisions about the care of individual patients, and the integration of best research evidence with clinical expertise and patient values. ${ }^{5}$ EBP is a process that consists of sequential steps: first, translating a clinical problem into answerable questions; second, tracking down, with maximum efficiency, the best research evidence; third, critically appraising the evidence for its methodological quality and usefulness; fourth, integrating the research evidence with clinical expertise and patient values and implementing it in practice; and fifth, evaluating the process and the result. ${ }^{5,6}$

\section{Background}

EBP is insufficiently implemented in routine practice. ${ }^{7-10}$ The time lag between the publication of research findings and their adoption in practice is estimated to be eight to thirty years. ${ }^{11,12}$ In clinical decision-making, nurses primarily rely on informal and experiential resources, and less on scientific research and protocols. ${ }^{13-15}$. Moreover, integrating the patient's perspective in EBP is challenging ${ }^{16}$ and research on the effects of patient involvement in EBP is scarce. ${ }^{17}$ Various factors at different levels stimulate or impede implementation ${ }^{18,19}$ including individual factors like nurses' basic research training, attitudes and beliefs. ${ }^{9}$ Munten et al. $^{20}$ published a review on the implementation of EBP, and concluded that implementation using action research was a promising approach, but that studies failed to explicitly report implementation strategies. Grimshaw et al. ${ }^{21}$ reviewed 41 systematic reviews on implementation strategies. They concluded that educational outreach and reminders might be promising, and that multifaceted interventions targeting different barriers to change are likely to be effective. Thompson et al. ${ }^{9}$ conducted a systematic review and concluded that education seemed to be effective, but only when combined with the education of local opinion leaders.

The literature thus shows that EBP is insufficiently integrated in routine nursing practice, and that its implementation is complex. While EBP is often implemented in an iterative and participatory manner, a systematic approach to its implementation is rare. As Rycroft-Malone et al. ${ }^{22}$ suggest, knowledge generation in EBP stems from four different types of evidence: research, clinical expertise, patients and local context of care. It is 
important to study the implementation of EBP in a natural context, as this yields information on all four types of evidence. The research question that arises is: How can EBP be systematically implemented jointly with front-line nurses in a clinical setting? The goal of this article is to describe the process of implementing EBP using participatory action research.

\section{Methods}

The method used in this study was a participatory action research design with cyclical activities involving observing, reflecting, planning and acting. The observing and reflecting phases provided information for subsequent planning. ${ }^{23}$ See Table 2.1 for an overview of the specific methods used in the different phases of action research. To critically review the existing situation, we started the observing phase with a working group consisting of the researcher (AM), four nurses of the lung unit, the unit's manager and a staff member of the hospital's educational department. We reflected on the current practice situation and the barriers and facilitators for implementing EBP, in order to select strategies for implementing EBP addressing barriers and facilitators, and the particular contextual situation regarding EBP. The nurses intended to use the development and implementation of a unit-specific EBP protocol for nursing discharge as a vehicle to implement EBP. We continuously evaluated the process in the working group by means of observing and reflecting, and made a summative evaluation at the end of the project.

\section{Rationale}

We chose an action research approach because it was compatible with the participatory and developmental nature of the study. Action research acknowledges the nature of a research study as a complex social process, and yields knowledge based on studies conducted within practical contexts. ${ }^{23}$ All those who participated in the study shared views, perceptions and ideas and contributed to change according to their expertise and knowledge.

\section{Setting}

The study was carried out in a lung unit of a rural hospital in the Netherlands. The lung unit has 18 beds and provides medium-complex care to patients with lung problems. Care is delivered to patients who need minor surgical procedures (e.g. thorax drainage), 
acute care (e.g. for acute exacerbations), chronic care (e.g. for chronic obstructive lung diseases) or palliative care.

Table 2.1 Methods and data collection in the different phases of action research.

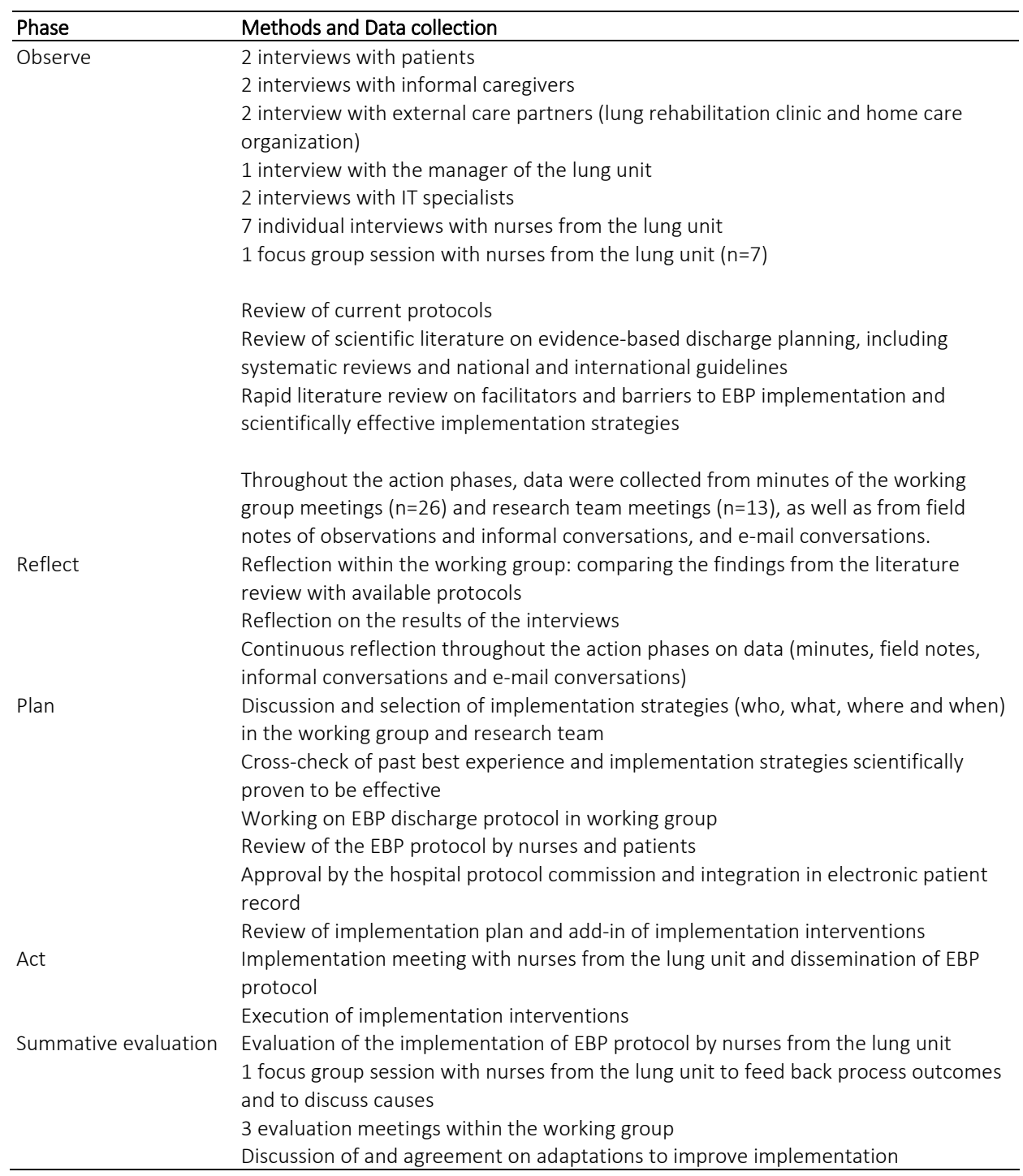

\section{Participants}

We used two sampling strategies: total population sampling and purposive sampling. ${ }^{24}$ Total population sampling was used for the implementation of EBP at the lung unit. All 
nurses of the lung unit $(n=14)$, including the manager $(n=1)$, participated in this study. Their ages ranged from 26 to 59 years. The mean age was 42 years. The number of years of work experience at this unit ranged from 5 to 43. All but four nurses worked parttime. All nurses were registered nurses with a medium-level vocational nursing training, which is lower than a bachelor's degree. None of the nurses had experience with or had been trained in applying EBP. A staff member of the educational department of the hospital also participated $(n=1)$. Purposive sampling was used to gain information about the current state of affairs in the practice situation, and about the barriers and facilitators for implementing EBP at the beginning of the study. In addition to all nurses of the ward $(n=14)$, information technology (IT) specialists $(n=2)$ and nurses of external care partners $(n=2)$, such as home care services and rehabilitation clinics, took part. Patient participants were drawn from the hospital's client council $(n=8)$ and patients who had recently been discharged from the lung unit $(n=2)$. The main informal caregivers $(n=2)$ of the discharged patients were also interviewed.

All participants received information about the study and provided consent for their participation. The institutional board of Zuyd University and Laurentius Hospital Roermond gave permission to carry out the study.

\section{Roles of the researcher, the working group and the research team}

Together with the working group, the researcher (AM) worked to facilitate change and the implementation process. We reflected on the findings throughout the action phases, first within the working group and afterwards at the unit. These reflections and the subsequent planning and observations of actions were closely related to the problems encountered in the routine practice of implementing EBP at the lung unit.

The researcher's role varied between the stages of the research. She always functioned as a partner in the process. In addition, she carried out certain tasks, suggested solutions, analysed data, and sometimes had to function as the main driver for the project. The researcher also provided the link with the research team. The research team functioned as an advisory board and had four members, all of them experts in implementing EBP. They followed the study and were responsible for overseeing the process; they gave advice and reviewed several documents and tools produced by the project.

\section{Data collection}

The data were continuously collected during a 24-month period in 2010-2012. A multimethod process of data collection was used, which emphasized iteration between planning, acting, observing and reflecting, as recommended in action research. We 
reviewed the literature and collected qualitative data by means of in-depth interviews, focus group discussions, field notes of informal conversations and observations, e-mail conversations, and minutes of the working group meetings and the advisory meetings of the research group. The purpose of literature reviews in action research is to ascertain what is already known about a topic, as well as to generate ideas for changing practice. $^{23,25}$ Interviews and focus group discussions were tape-recorded and transcribed. Where this was not possible, extensive field notes were made. See Table 2.1 for an overview of the data collection methods in the various action research phases.

\section{Data analysis}

We analysed qualitative data using an interpretive constant comparative approach. ${ }^{26} \mathrm{We}$ identified and explored emergent themes arising from the data and from participants' discussions during each iterative action phase in the EBP implementation process. The researcher developed the initial coding by identifying words, sentences or segments of data. This was followed by focused coding using the most significant or frequent initial codes to sort, synthesize, integrate and organize large amounts of data. ${ }^{26} \mathrm{Next}$, the data were discussed in the working group and research team until stable themes developed. The findings were used to guide the implementation of EBP and inform subsequent activities. Multiple strategies were applied to ensure trustworthiness, see Table 2.2.

Table 2.2 Strategies to ensure trustworthiness reliability of results.

\begin{tabular}{|c|c|}
\hline Method triangulation: & $\begin{array}{l}\text { We used multiple methods of data collection: in-depth interviews, focus } \\
\text { group discussions, observations, written minutes and other documents. }\end{array}$ \\
\hline Researcher triangulation: & $\begin{array}{l}\text { Multiple researchers reflected on the methods and results of the phases of } \\
\text { the action study. We reviewed and discussed scientific and organizational } \\
\text { aspects of the study within the research team. }\end{array}$ \\
\hline Data triangulation: & $\begin{array}{l}\text { We used multiple data sources during the study to verify the results, such as } \\
\text { nurses, patients, informal caregivers, and various written documents, } \\
\text { reflective and analytical notes. }\end{array}$ \\
\hline Member check: & $\begin{array}{l}\text { We fed back and discussed data in meetings with the working group, for } \\
\text { example verifying the findings from the interviews and summative } \\
\text { evaluation. }\end{array}$ \\
\hline Thick description: & $\begin{array}{l}\text { We gave a rich description of the study context to enable readers to judge } \\
\text { whether the findings are transferable to other care contexts. }\end{array}$ \\
\hline
\end{tabular}

\section{Findings}

The main findings of the different phases of action research are described below as follows. For the observing phase: the initial situation, i.e. a description of the practice situation at the beginning of the project and literature on evidence-based discharge care 
and barriers and facilitators for the implementation of EBP. For the reflecting phase: project targets. For the planning phase: implementation strategies. For the acting phase: tailored interactive outreach training and an adapted EBP discharge protocol. Finally, we describe the main results of the summative evaluation.

\title{
Observing phase: the initial situation
}

\section{Description of the practice situation: EBP and evidence-based discharge planning}

The nurses explained in the interviews that they had little knowledge of EBP and little experience with its systematic implementation. Nurses wanted to implement EBP by developing and implementing a unit-specific EBP protocol for nursing discharge planning. The nurses themselves proposed to use the discharge protocol as a vehicle to implement EBP. Existing unit-specific protocols were rarely used on the ward, and discharge care was provided based on nurses' individual expertise, with much variation among nurses.

\begin{abstract}
"We have a lot of protocols. I do not remember how many. They are in a file in our office but we rarely use them. For discharge planning every nurse has her own way of working. It differs a lot." (interview with nurse)
\end{abstract}

All current unit-specific protocols were based on best practice, mostly describing the current mode of working. There were 17 disease-specific protocols, and five of them included some information about discharge. The clinical problem which arose was that patients did not receive discharge care that had been standardized and found to be effective (step 1 of EBP).

The interviews with the patients and informal caregivers made it clear that they wanted to be informed about discharge at least 24 hours in advance, and that they had not received sufficient written and oral information. They often felt left alone with care issues in the home setting, like the prevention of secondary lung infections.

\footnotetext{
"Since I have been home from hospital I am not sure if I am allowed to take a walk. I was admitted for being short of breath. I am afraid I will catch a flu or pneumonia if I spend time outdoors. They [nurses] did not provide me with information on this. I feel quite left alone" (interview with discharged patient)
} 
External care partners said that information in the hand-over documents was partly incomplete and seemed to have been hastily written. Patients were insufficiently informed about after-care. Nurses at the lung unit and the manager said that the discharge process was unstructured, that the patients' discharge needs were insufficiently assessed, that the discharge procedure was not regularly evaluated and that there was no exit evaluation.

"Our patients just go home. There is no structured procedure the nurses follow. They do whatever they think is right. There is no exit interview, even worse, no evaluation of the discharge process at all." (interview with manager)

The IT specialists did not identify specific shortcomings but recommended using the electronic patient record as a vehicle for implementation.

\section{Literature on evidence-based discharge care}

We were unable to find literature focusing on discharge interventions for patients with lung problems. We found no guidelines, only one meta-analysis, ${ }^{27}$ several systematic reviews ${ }^{28-31}$ and a review. ${ }^{32}$ These articles either related to specific health problems (e.g. congestive heart failure), or to specific populations (e.g. older people). Generic components which, according to the literature, seemed to be effective in evidencebased discharge care were: structured and comprehensive discharge intervention, ${ }^{27-31}$ post-discharge care, ${ }^{27,28,30}$ educational interventions ${ }^{28,30}$ and participation of informal caregivers in the discharge process. ${ }^{32}$

\section{Barriers and facilitators for implementation}

The barriers and facilitators for the implementation of EBP among nurses are summarized in Table 2.3. The main barriers were that nurses had little knowledge of $E B P$, and that their English reading proficiency was poor. Many nurses had a more or less negative attitude towards EBP. The main facilitators were that the nurses wanted to deliver high quality care and that they were critical, enthusiastic and open to innovation. The nurses were also enthusiastic about developing and implementing a discharge protocol. This made EBP more practice-oriented, whereas before they had perceived it as an abstract concept. 
Table 2.3 Facilitators and barriers, and implementation strategies.

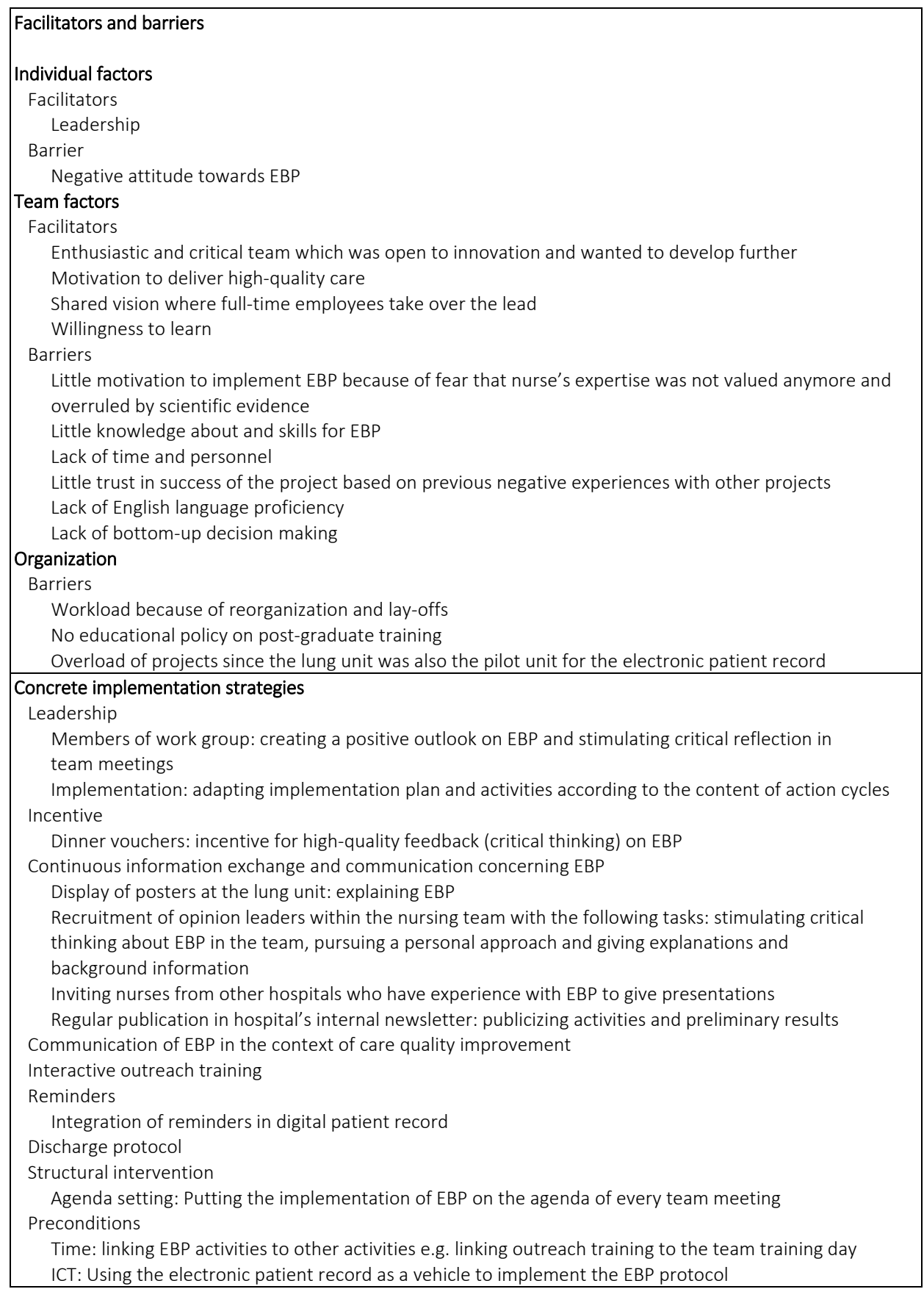


During the development of the discharge protocol, an additional barrier emerged: some nurses felt overruled by the scientific evidence and feared that their expertise was not valued.

"[name] reported in the meeting [of the working group] that she had had a talk with some nurses in the coffee corner last week. They felt that their expertise as nurses was being undermined by the discharge protocol. [name another nurse] nodded. She mentioned another occasion where two nurses expressed the same view." (field note from working group)

\section{Reflecting phase: formulation of project targets}

The reflecting phase resulted in three project targets, which were jointly formulated by the nurses and the researcher:

1. To develop a discharge protocol by integrating evidence from scientific literature, nurses' expertise, and patient experiences, tailored to the situation at the lung unit and applicable to all patient situations. This protocol should be developed in a format compatible with existing formats used at the hospital.

2. To disseminate and implement the discharge protocol at the lung unit and to integrate it in the electronic patient record.

3. To ensure that nurses at the lung unit are knowledgeable about the principles of EBP and know how to perform it.

Reflections on the additional barrier that emerged during the development of the discharge protocol, i.e. that some nurses felt overruled by the scientific evidence, resulted in adjustments to the first project target, specifically stating that a discharge protocol should be developed starting from the nurses' expertise with discharge, while subsequently incorporating aspects from the interviews and evidence from the literature.

\section{Planning phase: Implementation strategies}

Given the barriers and facilitators identified, the major implementation strategies planned were to develop and carry out a tailored interactive outreach training programme and to develop and implement an evidence-based discharge protocol.

Other implementation strategies that were planned included: members of the working group fostering a positive outlook on EBP and stimulating critical reflection in team meetings (Leadership), dinner vouchers as an incentive for high-quality feedback on EBP, continuous information exchange and communication concerning EBP, reminders, putting the implementation of EBP on the agenda of all team meetings (Structural 
intervention) and creating the necessary preconditions, for example using the electronic patient record as a vehicle to implement the EBP protocol. For an overview of all planned implementation strategies see Table 2.3.

\section{Acting phase: Executing major implementation strategies}

\section{Tailored interactive outreach training}

The tailored interactive outreach training programme adopted the $\mathrm{PI}$ (Patient/Population and Intervention) method instead of the PICO (Patient/Population, Intervention, Comparison, Outcome) method in step 1 of the EBP process. Step 2 involved using national Dutch websites which published reliable nursing and multidisciplinary clinical practice guidelines, while less use was made of international publications like systematic reviews and the Cochrane data. In step 3, four critical appraisal questions derived from the Appraisal of Guidelines for Research \& Evaluation (AGREE) instrument ${ }^{33}$ were used to assess the quality of the evidence. Box 2.1 provides a description of the interactive outreach training.

Box 2.1 Goal and description of the tailored outreach training programme.

The aims were (a) providing interactive outreach training so that EBP was immediately applicable for nurses, (b) translating and adapting EBP activities so that beginners with little preexisting knowledge could practice it and (c) using Dutch guidelines.

The first part dealt with the definition of EBP, the goal, barriers and EBP competencies. The second part explained the steps of EBP. In the first EBP step we explained the PI method, which is a variant of the P (patient/population) I (intervention) C (comparison) O (outcome) method. P was defined as the problem or patient population and I as intervention. The PI terms were also the search terms (e.g. COPD and high energy intake). In the second EBP step we recommended reliable Dutch databases to track guidelines. We used databases that published nursing guidelines but also multidisciplinary guidelines. We showed the nurses how to find these sources on the web. In the third EBP step we explained how to critically appraise the guidelines. We used an adaptation of the Appraisal of Guidelines for Research and Evaluation II (AGREE) instrument ${ }^{33}$ and included four critical appraisal questions: Do you understand the content of the guideline? Does the guideline provide an answer to the PI question? Is the guideline up to date (not older than 5 years)? Can the recommendations be put into practice? We created a yes/no answer option and made it a cut-off * criterion* that all questions needed to be answered in the affirmative if the guideline was to be included. Next we explained the levels of evidence. Systematic reviews were labeled as level A, RCTs as level B, observational studies as level C and expert committees as level D. However, we did not explain the research design, but the strengths of evidence. We simplified the explanations: "level A, strong" was explained as: it is evident that..., "level B, medium": it is assumed that ..., "level C, weak": it is likely that....., and "level D, no evidence": the guideline working group is of the opinion that.... These formulations were adopted from the Dutch Institute for Healthcare Improvement. ${ }^{34}$ In the fourth EBP step we highlighted that nurses needed to provide a concise conclusion about the results, determine if the results could be put into practice at their own unit, provide a recommendation and finally communicate with the unit to update existing protocols. In the final EBP step we explained that an evaluation should take place from the patients' point of view and from the nurses' perspective. In the last part we presented a case about a patient with a lung problem. Nurses formed small groups and jointly worked through the first four EBP steps. The training course ended with a plenary discussion. 


\section{Adjusted EBP discharge protocol}

As a result of the adjusted first project target (See Reflecting phase), the adjusted discharge protocol was based on the nurses' best-practice mode of working, as well as on evidence from the literature and aspects that emerged in the interviews with representatives, patients, informal caregivers and external care partners.

See Table 2.4 for the major components of the protocol. The protocol was linked to the electronic patient record.

Table 2.4 Major components of the discharge protocol.

\begin{tabular}{ll}
\hline Exit evaluation & $\begin{array}{l}\text { Discharge visit before discharge (if possible with patient and } \\
\text { informal caregiver) }\end{array}$ \\
& Discharge letter \\
& Handing over documents for external partners \\
Patient education & Medication (oral and written information) \\
Follow-up & Lifestyle interventions (oral and written information) \\
Timely communication of discharge date & $\begin{array}{l}\text { Follow-up appointments } \\
\text { hours before discharge }\end{array}$ \\
Electronic patient record & $\begin{array}{l}\text { Registration of discharge components in electronic patient } \\
\text { record }\end{array}$ \\
\hline
\end{tabular}

\section{Summative evaluation}

\section{Implementation of EBP}

Several nurses remained reluctant about EBP. The main reasons were that they considered EBP to be "theoretical", that they were concerned that their professional expertise was being overridden, and that they perceived a lack of time. Others mentioned time constraints and not seeing any benefit to their professional performance. Few nurses made use of the websites recommended in the outreach training programme.

At first, the nurses experienced EBP as very complicated and complex. After the outreach training programme, however, they felt confident about reviewing Dutch sources of guidelines. They explained that the project stimulated their critical thinking about EBP and care rituals. They were positive about using the learning-by-doing 
approach, which is inherent in action research, to design the discharge protocol. This helped them to apply EBP and implement it directly. It was concluded that the targets of this project had been partly met, although true implementation of EBP and fostering an EBP culture at the lung unit would require some further action.

"There is a long way to go to implement evidence-based practice. This project was only the beginning. There is a need to enact an EBP culture where nurses integrate science in their daily routines. We need a culture change away from solely relying on individual expertise." (minutes of working group meeting)

\section{Implementation of the discharge protocol}

The nurses carried out care activities in accordance with the discharge protocol, but did not register their activities in the electronic patient record immediately and according to the protocol. They registered care activities at the end of the day, while the electronic patient records of discharged patients were closed in the morning. The registration of discharge care activities in the electronic patient record was perceived as complex.

\footnotetext{
"Discharge registration has become quite difficult. We must open a lot of additional windows and enter a lot of ticks. In some instances it is not clear to me where I should find the right window and what I need to tick. In view of the time I need to find the right windows, I do not register at all." (focus group session with nurses)
}

The patient exit evaluation on the ward was performed shortly before the patient left, and nurses had to organize some discharge care activities at the last minute, which were not registered either.

Finally, the summative evaluation also resulted in plans to continue EBP. This involved, firstly, one nurse taking over the lead and continuing the working group sessions; secondly, using a tool to review existing protocols based on the principles of EBP in order to adapt existing protocols step-by-step towards the EBP approach; and finally starting the development of another protocol based on EBP. 


\section{Discussion}

We used a participatory action research design to explore the implementation of EBP. Munten et al. ${ }^{20}$ state that action research might be a promising method for implementing EBP, though they were unable to conclude that action research is more successful than designs which are less iterative and not based on partnerships. The advantage we experienced is that we were able to adapt the implementation of EBP to the setting and to tailor the implementation strategies. In this way we empowered nurses to participate in the process. ${ }^{23}$ It was the nurses themselves who proposed using the discharge protocol as a vehicle to implement EBP.

Facilitators and barriers we identified in this study were in agreement with systematic reviews concerning the implementation of guidelines and protocols in the nursing setting. ${ }^{35-37}$. Furthermore, Baker et al. $^{38}$ concluded in a systematic review that interventions tailored to prospectively identified barriers are more likely to improve professional practice than no intervention or the dissemination of guidelines. In our study we tailored the interventions to the barriers we identified. However, there is as yet insufficient evidence on the most effective approaches to identifying barriers and on tailoring interventions. ${ }^{38}$ In this study we used the knowledge and clinical expertise of members of the working group ${ }^{22}$ to tailor interventions that had been reported in the literature to be effective.

It proved possible to implement a discharge protocol in a clinical setting. We used nurses' practical expertise as a starting point for the discharge protocol. The situation in nursing is such that patients rarely participate in the implementation process. ${ }^{39} \mathrm{We}$ incorporated the patient perspective by consulting patients for the description of the current practice of the discharge procedure, and we asked them to provide feedback on the discharge protocol, which we then used to develop the unit-specific protocol. As regards scientific evidence, we had to make considerable adjustments, which raised the question whether we still met the conditions of EBP concerning scientific evidence? We are positive in this regard, as we complied with the methodological requirements of EBP, even though we tailored activities within the steps to the practical context. Yet, we did in a way start with naïve ideas, as we thought that we could translate the steps of EBP as described by. 5,40 These steps do not seem to be suitable for front-line nurses with medium-level vocational training. In our setting, the average age of nurses was over 40 years, none of them had a bachelor's or master's degree or training in EBP, and their English reading proficiency was poor. Our setting reflects current trends in the nursing work force in general. ${ }^{41,42}$

The implementation of EBP in our study was in agreement with the literature, in which EBP is often about the implementation of scientific evidence or clinical practice 
guidelines in an organization or at unit level ${ }^{10,43}$ and not so much about the use of scientific evidence and clinical practice recommendations in the decision-making process for individual patients, ${ }^{44}$ as it was originally defined by Sackett et al. ${ }^{5}$

After an implementation project is completed, there is always a high risk of reverting to old routines. Some conditions described in the literature ${ }^{19,45}$ to ensure sustainability were met, like one nurse taking over the lead and continuing the working group sessions. However, a culture shift toward EBP, which was perceived necessary to really implement EBP, was not yet achieved. Most authors in healthcare emphasize that organizations have to develop an "EBP culture", in which continuous learning, teamwork and patient focus are central, in order to really achieve a change in patient care. ${ }^{19,46}$

\section{Strengths and limitations}

The strengths of action research are the cyclical process and the partnership between the researcher and those involved in the process. The cyclical approach to EBP implementation produced situation-specific knowledge.

Although we do not claim empirical transferability, we propose that we have achieved transferability of knowledge, which means that the reader decides which knowledge he/she perceives to fit his/her own setting and to be worth transferring. We have developed some initial insights about adapting EBP so as to make it more practiceoriented. Our study did not specifically tailor the discharge protocol to the needs of individual patients as regards decision making about individual care.

We used purposive sampling to gain information about the initial situation. We interviewed persons from different groups of stakeholders (e.g. nurses, patients and external care partners) in order to incorporate their views. We may not have reached data saturation for all groups of stakeholders, however. For example, only two nurses from external care partners were interviewed. Furthermore, although we had planned to involve recently discharged patients in the study, only two patients agreed to participate. Other patients said it would be too much of a burden to them, and we respected this. The members of the client council, however, were very willing to participate, so we included all of them in the study to make sure that patient values as a component of EBP were truly part of the EBP implementation.

The approach we chose was time-consuming. From the perspective of implementation efficiency, this might perhaps not be the preferred method. Another problematic aspect is the focus of the discharge protocol. It seems to have added to the complexity of implementing EBP compared to situations where nurses might opt for a simpler subject, such as improving hand hygiene. 


\section{Conclusion}

Our study has generated insights into the development of practice-informed implementation, and yielded knowledge from front-line nurses who implemented EBP. Action research provides an opportunity to empower nurses and to tailor EBP to the practice context to do justice to the nature of this design. We learned that the steps of EBP were difficult for nurses to apply, especially for nurses with a medium-level vocational training. It seems that there is a need for a simplified and pragmatic method of EBP next to the full-blown academic version. Our study might provide some preliminary insights into what a pragmatic method of EBP could perhaps look like. Although we were able to implement a discharge protocol, the nurses felt that true implementation of EBP would require introducing an EBP culture.

\section{Relevance to clinical practice}

Strategies to implement EBP should as much as possible be fine-tuned and tailored to the practice situation. Adjustment of the academic model of EBP to develop a more pragmatic method seems necessary to introduce EBP into clinical practice. The use of scientific evidence in clinical practice should be facilitated by using pre-appraised evidence, like clinical practice guidelines.

The situation in nursing is such that patients rarely participate in the implementation of EBP. We involved patients by asking them to provide information and consulting them at unit level. However, to provide high-quality patient-centred care, patients must also have a voice in clinical decision-making. In the context of EBP, scientific evidence should not claim priority over the patient's wishes and over professional knowledge. Rather, we consider it relevant for clinical practice to implement the integration of scientific evidence with clinical expertise and patient values in nurses' clinical decision making at the individual patient level.

\section{What does this paper contribute to the wider global clinical community}

- A participatory action research design has generated insights into ways of implementing EBP in a tailored and systematic manner.

- The content of EBP must be adapted to the specific competencies of nurses working in a clinical setting.

- Knowledge of and implementation experience with EBP in a natural setting contribute to practice-informed implementation science. 


\section{References}

1. Thomas L, Cullum N, McColl E, Rousseau N, Soutter J, Steen N. Guidelines in professions allied to medicine. Cochrane Database Syst Rev. 1999; 1, CD000349. DOI: 10.1002/14651858. CD000349.

2. Australian Nursing and Midwifery Council (ANMC). Code of Professional Conduct for Nurses in Australia. ANMC, Canberra, Australia. 2003.

3. Nursing and Midwifery Council (NMC). The NMC code of Professional Conduct: Standards for Conduct, Performance and Ethics. NMC, London. 2004.

4. Dutch Ministry of Health, Welfare and Sport (VWS). Social challenges in Health and Welfare. VWS, The Hague. 2009.

5. Sackett DL, Straus SE, Richardson WS, Rosenberg WM, Haynes RB. Evidence-based Medicine: How to Practice and Teach EBM. Churchill Livingstone, Edinburgh. 2000.

6. Sackett DL, Rosenberg WMC. The need for evidence-based medicine. J R Soc Med. 1995;88:620-4.

7. Schuster M, McGlynn E, Brook R. How good is the quality of health care in the United States ? Milbank Q. 1998;76:517-63.

8. Grol R, Baker R, Moss F. Quality improvement research: understanding the science of change in health care. British Medical Journal Books, London. 2004.

9. Thompson D, Estabrooks CA, Scott-Finlay S, Moore K, Wallin L. Interventions aimed at increasing research use in nursing. A systematic review. Implement Sci. 2007;2:1-16.

10. Squires JE, Hutchinson AM, Bostrom A, O'Rourke HM, Cobban SJ, Estabrooks CA. To what extent do nurses use research in clinical practice ? A systematic review. Implement Sci. 2011;6:1-43.

11. Bostrom J, Wise L. Closing the gap between research use and practice. J Nurs Adm. 1994;24:22-7.

12. Landrum BJ. Marketing innovations to nurses: part 2: marketing's role in the adoption of innovations. J Wound Ostomy Continence Nurs. 1998;25:227-32.

13. Spenceley SM, O'Leary K, Chizawsky LLK, Ross AJ, Estabrooks CA. Sources of information used by nurses to inform practice: An integrative review. Int J Nurs Stud 2008;45:954-70.

14. Rycroft-Malone J, Fontenla M, Seers K, Bick D. Protocol-based care: the standardisation of decisionmaking? J Clin Nurs 2009;19:1490-500.

15. Traynor M, Boland M, Buus N. Autonomy, evidence and intuition: nurses and decision-making. J Adv Nurs 2010;66:1548-91.

16. Strauss SE, Jones G. What has evidence based medicine done for us? BMJ 2004;329:987-8.

17. Nilsen ES, Myrhoug HT, Johanson M, Oliver S, Oxman AD. (2006) Methods of consumer involvement in developing healthcare policy and research, clinical practice guidelines and patient information material. Cochrane Database Syst Rev. 2006;3:1-34.

18. Wensing $M$, Bosch M, Grol R. The knowledge to action cycle: developing and selecting knowledge in action interventions. Can Med Assoc J. 2010;82:E85-8.

19. Grol R, Wensing M, Eccles M, Davis D. Improving Patient Care: The Implementation of Change in Health Care. John Wiley \& Sons, Ltd, West Sussex. 2013.

20. Munten G, Bogaard VD, Cox K, Garretsen H, Bongers I. Implementation of evidence-based practice in nursing using action research: a review. Worldviews Evid Based Nurs. 2010;7:135-57.

21. Grimshaw JM, Shirran L, Thomas R, Mowatt G, Fraser C, Bero L, Grilli R, Harvey E, Oxman A, O'Brian MA. Changing provider behaviour. An overview of systematic reviews of interventions. Medical Care 2001;38:(Suppl. 2), II-2-II-45.

22. Rycroft-Malone J, Seers K, Titchen A, Harvey G, Kitson A, McCormack B. What counts as evidence in evidence-based practice? J Adv Nurs 2004;47:81-90.

23. Koshy E, Koshy V, Waterman H. Action research in healthcare. Sage, London. 2011.

24. Polit DF, Beck CT. Nursing Research. Generating and Assessing Evidence for Nursing Practice., 9th edn. Wolters Kluwer, Lippincott, Williams \& Wilkins, Philadelphia, PA. 2012.

25. Mitchell EA, Conlon AM, Armstrong M, Ryan AA. Towards rehabilitative handling in caring for patients following stroke: a participatory action research project. J Clin Nurs 2005;14:3-12.

26. Charmaz K. Constructing grounded theory. A practical guide through qualitative analysis. Sage, London. 2006. 
27. Phillips CO, Wright SM, Kern DE, Singa RM, Schepperd S, Rubin HR. Comprehensive discharge planning with postdischarge support for older patients with congestive heart failure. JAMA 2004;291;1358-67.

28. Parker SG, Peet SM, McPherson A, Cannaby AM, Abrams K, Wilson A, Lindsay J, Parker G, Jones DR. (2002) A systematic review of discharge arrangements for older people. Health Technol Assess. 2002;6:4.

29. Richards S, Coast J. Interventions to improve access to health and social care after discharge from hospital: a systematic review. J Health Serv Res Policy. 2003;8:171-9.

30. Mistiaen P, Franke AL, Poot E. Interventions aimed at reducing problems in adult patients discharged from hospital to home: a systematic review. BMC Health Serv Res. 2007;7 (47): 1-19.

31. Shepperd S, McClaran J, Phillips CO, Lannin NA, Clemson LM, McCluskey A, Cameron ID, Barras SL. Discharge planning from hospital to home. Cochrane Database Syst Rev. 2010; 1: CD000313. DOI: 10.1002/14651858.CD000313.pub3.

32. Bauer M, Fitzgerald L, Haesler E, Manfrin M. Hospital discharge planning for frail older people and their family. Are we delivering best practice? A review of evidence. J Clin Nurs. 2009;18:2539-46.

33. Brouwers MC. Appraisal of Guidelines for Research \& Evaluation instrument II. The Agree Next Steps Consortium, Hamilton, Canada. 2009.

34. Dutch Institute for Healthcare Improvement (CBO) (2007) Development of Evidence-based Guidelines: Instructions for developers. CBO, Utrecht.

35. Estabrooks CA, Floyd JA, Scott-Findlay S, O'Leary KA, Gushta M. Individual determinants of research utilization: a systematic review. J Adv Nurs. 2003;43:506-20.

36. Ploeg J, Davies B, Edwards N, Gifford W, Miller PE. Factors influencing best-practice guideline implementation: lessons learned from administrators, nursing staff, and project leaders. Worldviews Evid Based Nurs. 2007;4:210-9.

37. Eizenberg MM. Implementation of evidence-based nursing practice: nurses' personal and professional factors? J Adv Nurs 2010;67:33-42.

38. Baker R, Camosso-Stefinovic J, Gillies C, Shaw EJ, Cheater F, Flottorp S, Robertson N. Tailored interven tions to overcome identified barriers to change: effects on professional practice and health care outcomes. Cochrane Database of Systematic Reviews, Issue 3. Article ID: Art. No.: CD005470. 2010. DOI: 10.1002/14651858. CD005470.pub2.

39. Van Achterberg T, Schoonhoven L, Grol R. Nursing implementation science: How evidence-based nursing requires evidence-based implementation. J Nurs Scholarsh 2008;40(4):302-10.

40. Sackett DL, Rosenberg WM, Gray JA, Haynes RB, Richardon WS. Evidence-based medicine: what is it and what it isn't. BMJ. 1996;312:71-2.

41. Buerhaus P, DesRoches C, Donelan K. Nursing Trends: 2007. Key facts about a changing workforce. Johnson \& Johnson, New Brunswick, NJ. 2007.

42. Melnyk BM, Fineout-Overholt E. The evidence-based practice beliefs and implementation scales psychometric properties of two new instruments. Worldviews Evid Based Nurs. 2008;5:208-16.

43. Gawlinski A, Rutledge D. Selecting a Model for Evidence-Based Practice Changes: A Practical Approach. AACN Adv Crit Care. 2008;19:291-300.

44. Barratt A. Evidence based medicine and shared decision making: the challenge of getting both evidence and preferences into health care. Patient Educ Couns 2008;73:407-12.

45. Gruen RL, Elliott JH, Nolan ML. Sustainability science: an integrated approach for health-programme planning. Lancet 2008;372:1579-89.

46. Ferlie EB, Shortell SM. Improving the quality of health care in the United Kingdom and the United States: a framework for change. Milbank Q. 2001;79:281-315. 



\section{Chapter}

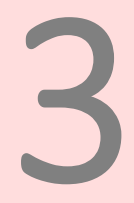

Teaching and implementing evidence-based practice in a hospital unit with mainly secondary vocational trained nurses: Lessons learned

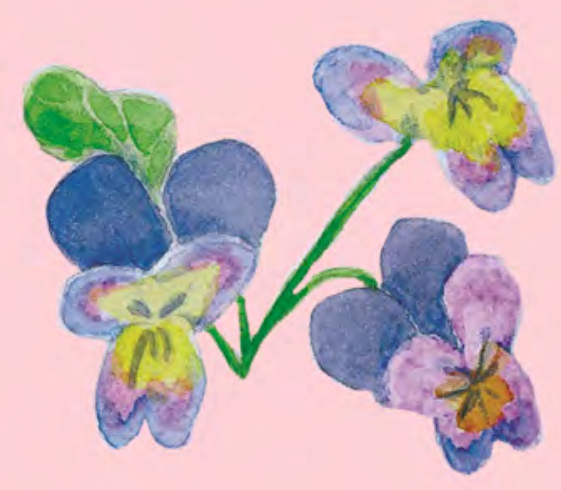

Jolanda H.H.M. Friesen-Storms Anna J.H.M. Beurskens

Gerrie J.J.W. Bours

The Journal of Continuing Education in Nursing 2017;48(9):407-412 


\section{Abstract}

Evidence-based practice was systematically implemented using the implementation model by Grol et al. Barriers and facilitators for change were diagnostically analyzed. Implementation strategies were directed at the barriers. The two main implementation interventions were: (1) a tailored interactive outreach training course about EBP and (2) a patient case discussion based on evidence-based practice principles. We learned that it is important to simplify the five EBP steps to suit the level of education of the nurses: formulating PICO questions around major patient care topics such as pain, and having a search strategy focusing on (Dutch) clinical practice guidelines and other summarized evidence, such as systematic reviews and critically appraised topics. We advised the hospital management to appoint nurse practitioners or other nurses with a Master's degree to assist the nurses on the ward with the EBP process steps, especially regarding efficiently searching the research literature, and critically appraising the evidence. 


\section{Introduction}

Nurses on the Advisory Board (VAR) of a rural hospital in in a rural Hospital in the South of the Netherlands and researchers of the Research Centre on Autonomy and Participation of Persons with a Chronic Illness at Zuyd University, wanted to improve the routine use of evidence-based practice (EBP) by nurses at the hospital. The pulmonary unit, a preceptor ward, was chosen as the first ward to start implementing EBP. The unit has 44 beds, and accommodates patients with different pulmonary diseases. Sixty nurses (40 full-time equivalents) worked on the ward. Four nurses (7\%) on the ward had a Bachelor's degree. The other nurses were RNs with 4 years of secondary vocational training. The RN degree based on 4 years of secondary vocational training is equivalent to the American Associate Degree Nurses. ${ }^{1}$ Although educational levels and training of nurses in the Netherlands differ, in daily clinical nursing practice and in existing law, the nursing degrees are not clearly differentiated as regards direct patient care. ${ }^{2}$

This paper describes the systematic approach used to teach and implement EBP on this pulmonary unit and the lessons learned.

\section{Description of strategy}

EBP was systematically implemented using the implementation model by Grol, et al. ${ }^{3}$ Barriers and facilitators for change were diagnostically analyzed by semi-structured interviews and by means of a questionnaire consisting of the EBP Barriers and Implementation Scales. ${ }^{4}$ These barriers and facilitators were used as a basis for selecting different implementation strategies and interventions at individual, social (team) and organizational levels (Table 3.1). Educational, motivational and organizational strategies were operationalized by developing two main implementation interventions: (1) a tailored interactive outreach training course about EBP and (2) a systems approach, described by Vermeulen et al., ${ }^{5}$ as, "the patient case discussion meeting based on EBP principles," used as a vehicle to implement and embed EBP in daily practice. The development and content of the two interventions are described in more detail below. 
Table 3.1 Implementation strategies and interventions targeting the barriers and facilitators at different levels.

\begin{tabular}{|c|c|c|}
\hline Barriers and Facilitators & $\begin{array}{l}\text { Implementation } \\
\text { Strategies }\end{array}$ & Interventions \\
\hline \multicolumn{3}{|l|}{ Individual } \\
\hline Knowledge deficit & Educational strategies & $\begin{array}{l}\text { Tailored interactive outreach } \\
\text { training for preceptor nurses } \\
\text { and nurses of the pulmonary } \\
\text { unit } \\
\text { Coaching of other nurses by } \\
\text { nurses who received training }\end{array}$ \\
\hline $\begin{array}{l}\text { Not using the EBP process in daily } \\
\text { practice }\end{array}$ & $\begin{array}{l}\text { Motivational and organizational } \\
\text { strategies }\end{array}$ & $\begin{array}{l}\text { A systems approach involved } \\
\text { the "patient case discussion } \\
\text { meeting" as a vehicle to } \\
\text { implement EBP }\end{array}$ \\
\hline Not having a critical attitude & $\begin{array}{l}\text { Motivational and organizational } \\
\text { strategies }\end{array}$ & $\begin{array}{l}\text { Stimulating nurses to think } \\
\text { about problems in daily care as } \\
\text { part of the patient case } \\
\text { discussion meetings }\end{array}$ \\
\hline $\begin{array}{l}\text { Not convinced of the importance } \\
\text { of EBP }\end{array}$ & Motivational strategies & $\begin{array}{l}\text { Informing nurses about EBP and } \\
\text { the patient case discussion } \\
\text { during a meeting } \\
\text { Formal and informal leaders } \\
\text { creating commitment }\end{array}$ \\
\hline $\begin{array}{l}\text { Feeling insecure about attending } \\
\text { patient case discussion meetings }\end{array}$ & Motivational strategies & $\begin{array}{l}\text { Planning the first patient case } \\
\text { discussion meeting as an } \\
\text { informal lunch meeting }\end{array}$ \\
\hline \multicolumn{3}{|l|}{ Social: team } \\
\hline $\begin{array}{l}\text { Used to working with hospital- } \\
\text { specific protocols }\end{array}$ & $\begin{array}{l}\text { Organizational strategies and } \\
\text { educational strategies }\end{array}$ & Linking EBP to protocols \\
\hline $\begin{array}{l}\text { Presence of innovators and early } \\
\text { adopters }\end{array}$ & Motivational strategies & $\begin{array}{l}\text { Addressing innovators and early } \\
\text { adopters }\end{array}$ \\
\hline $\begin{array}{l}\text { Preceptor nurses linked to hospital } \\
\text { units }\end{array}$ & Educational and motivational strategies & $\begin{array}{l}\text { Training preceptor nurses first } \\
\text { to help them train and coach } \\
\text { nurses in hospital units }\end{array}$ \\
\hline \multicolumn{3}{|l|}{ Organization } \\
\hline $\begin{array}{l}\text { No access to research literature } \\
\text { and research databases }\end{array}$ & Organizational strategies & $\begin{array}{l}\text { Providing access to research } \\
\text { databases and literature }\end{array}$ \\
\hline Time is a constraint & Organizational strategies & $\begin{array}{l}\text { Integrating the patient case } \\
\text { discussion meetings with other } \\
\text { patient-focused meetings } \\
\text { Consulting the ward manager }\end{array}$ \\
\hline
\end{tabular}




\section{Tailored interactive outreach training}

The interactive outreach training course was developed using the EBP process steps as a common guideline (See Table 3.2). We used the three modes of performing EBP proposed by Straus and Green ${ }^{6}$ to shape the training course. In the "doing" mode, nurses have the skills to perform the first four steps of EBP; in the "using" mode the critical appraisal step is eliminated and the literature search is restricted to rigorously pre-appraised resources; in the "replicating" mode, the recommendations of respected EBP leaders are followed (abandoning at least the search for evidence and its detailed appraisal).

Table 3.2 Process and steps of evidence-based practice. ${ }^{7,8}$

\begin{tabular}{ll}
\hline Step & Process \\
\hline Step 1 & Formulate answerable questions \\
Step 2 & Search efficiently for the best research evidence \\
Step 3 & Critically appraise the research evidence \\
Step 4 & Integrate the research evidence, clinical expertise, and patient's values into actual practice, and \\
Step 5 & Evaluate the process and result \\
\hline
\end{tabular}

Since preceptor nurses, who are educators within a hospital, with a Bachelor's degree, are important role models and facilitators in coaching and implementing EBP, ${ }^{9}$ we first aimed to train the preceptor nurses of the hospital $(N=10)$ to become EBP "doers", and then to train eight nurses of the pulmonary unit to become at least "users" of EBP, while the other nurses were intended to become "followers". The training course for the preceptor nurses consisted of five two-hour meetings.

The first meeting of the training course for the preceptor nurses dealt with an introduction on EBP, with special attention for formulating answerable questions. We taught them to distinguish between background and foreground questions. Background questions concern general information about a clinical issue (for example regarding the physiology of pain), whereas foreground questions focus on specific knowledge (for example a question regarding a valid pain instrument). Background questions need to be answered as a foundation for formulating answerable foreground questions. ${ }^{10}$ The students were taught to formulate all questions in the PICO format (Patient/problem, Intervention, Control, Outcome), a format which supports formulating answerable questions.

In the second meeting, the preceptor nurses were asked to formulate a PICO question based on a patient case. The Librarians gave basic PubMed training and the nurses searched the literature on the basis of their PICO questions. The Librarians helped them define English search terms by looking up the English translation of Dutch terms on an 
online dictionary and by teaching them how to find Medical Subject Headings (MeSH) in PubMed. Many evidence-based practice guidelines are available in English.

The third meeting provided them with tools for critically appraising the literature, by discussing the hierarchy of evidence of different research designs and by teaching the basic principles of different research designs. We taught them to use evidence-based practice guidelines and critically appraised topics, which are published in journals in the Netherlands (for example in the journal "Nursing"). We also taught them the basic questions to consider when appraising any study: (1) Are the results of the study valid? (Validity) (2) How reliable are the results? (Reliability) and (3) Will the results help me in caring for my patients? (Acceptability). We provided them with Dutch tool boxes with rapid critical appraisal questions for different research designs. ${ }^{11-17}$ For example, one of the tool boxes provides a checklist to appraise the validity of randomized clinical trials by asking questions like: Was the assignment of the patients to the intervention randomized?

The fourth meeting discussed evidence-based clinical practice guidelines and systematic reviews. The appraisal of the literature reporting on original research designs was considered too difficult for the preceptor nurses, so in the fifth meeting we focused on practicing the critical appraisal of a guideline linked to a patient case, using the Appraisal of Guidelines for the Research and Evaluation II (AGREE) instrument. ${ }^{18}$ The fifth meeting also discussed barriers and facilitators to EBP implementation on the ward.

The results of an evaluation of the training course for preceptor nurses led to adjustments being made to the course for the nurses of the pulmonary unit. (1) The teaching about the basic principles of different research designs was considered too difficult and omitted. (2) Reading English research literature was considered difficult, so we focused more on national (i.e. Dutch) evidence-based clinical practice guidelines and databases.

Eight nurses of the pulmonary unit attended the adjusted training course.

A problem that arose during the training of the nurses of the pulmonary unit was that in critically reflecting on care and formulating PICO questions, the nurses formulated many problems and PICO questions, which were very specific on details of care, and were unsuitable for the literature search. For example, do you place the reservoir of the drain on the same side or the opposite side of the drain port? To help them handle this problem we discussed how to prioritize questions according to a sequence of criteria from the literature ${ }^{19}$ : (1) Urgency regarding the patient's well-being; (2) Relevance for learning needs; (3) Feasibility of answering the question within the available time; (4) Prevalence of the question; (5) Personal interest. 


\section{Patient case discussion to implement EBP}

After the outreach training course, patient case discussion meetings, ${ }^{5}$ were organized as a vehicle to implement EBP into the pulmonary unit daily routines. The patient case discussion meeting is a systematic approach to implementing EBP. The meetings are systematically organized and facilitated on the ward. ${ }^{5}$ In these discussions, nurses themselves selected a patient case from daily practice in which they experienced problems of care. ${ }^{5}$ The nurses discussed and explored this patient case following the steps of EBP.

During the first meeting, the nurses formulated a PICO question about a problem they had experienced (Box 3.1). Next, two nurses who had attended the outreach training course searched PubMed. A nurse practitioner advised them on how to search for a national (Dutch) evidence-based clinical practice guideline related to their question. In a second meeting, nurses discussed the literature. In a third meeting, they again discussed the literature and formulated an implementation proposal.

Box $3.1 \quad$ Example of a patient case discussion.

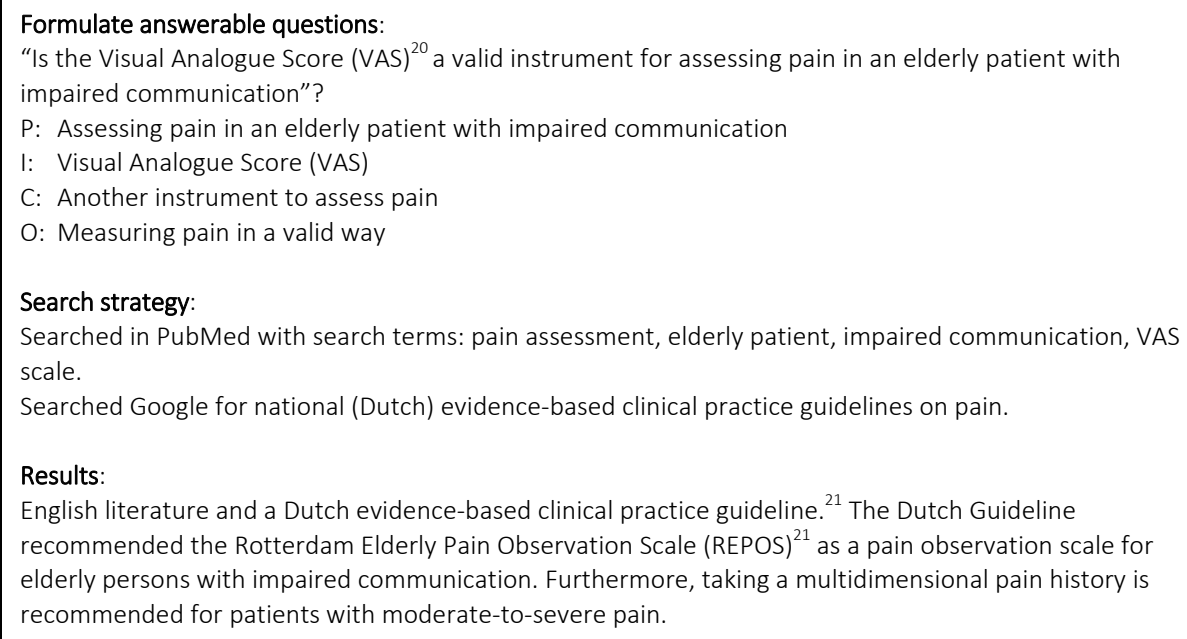

\section{Evaluation of the project}

The project was evaluated using a process evaluation. ${ }^{22}$ Eight nurses of the pulmonary unit were interviewed, all of whom had attended the outreach training course and/or patient case discussion meetings. 


\section{Results}

During the interviews the nurses mentioned several positive elements of the training and patient case discussions. Specifically, they reported being able to: (1) reflect critically on care, which the literature regards as a prerequisite for $\operatorname{EBP}^{23}$ (2) formulate a PICO question based on a patient-care problem they had experienced, (3) find English literature and Dutch guidelines related to the problem, and (4) translate the guideline recommendations into routine practice. As mentioned in the description of the tailored interactive outreach training course, a problem was that nurses formulated large numbers of very specific questions on details of care. By teaching them to prioritize questions, we got them to formulate more questions on major patient care topics.

Although nurses were able to search for English literature and guidelines, an efficient literature search remained difficult. Critically appraising the literature was considered too difficult for all nurses, even for those with a Bachelor's degree.

\section{Lessons learned}

Although we used a systematic approach to implement EBP, and the interventions were based on barriers and facilitators, implementation of EBP proved difficult. At first we were somewhat naïve in aiming for preceptor nurses to become "EBP doers" and eight nurses of the pulmonary unit to become at least "EBP users." The authors learned that it is important to simplify the five EBP steps to suit the level of education of nurses by formulating PICO questions around major patient care topics, such as pain, and having a search strategy focusing on Dutch evidence-based clinical practice guidelines and other summarized evidence, such as systematic reviews and critically appraised topics. The authors advised the hospital management to appoint nurse practitioners or other nurses with a Master's degree to assist the nurses on the ward with the EBP process steps, especially regarding efficiently searching the research literature, and critically appraising evidence.

Our findings are confirmed in a recent review ${ }^{24}$ regarding the state of readiness for evidence-based practice among nurses. It concludes that it is crucially important that nurse leaders and educators ensure that nurses working in daily practice have access to critically appraised and translated best evidence in a form that is relevant, practical, and adapted to the local clinical setting, such as evidence-based clinical practice guidelines. ${ }^{24}$ 


\section{Dissemination}

The lessons learned led us to adjust the training of Bachelor students in the nursing department of Zuyd University. Training in the first years will focus on finding and applying clinical practical guidelines for authentic patient cases. In the final years of training, students will learn to use PubMed and other English databases like Cinahl. Schools training secondary vocational nurses have been advised to focus on searching and applying (Dutch) clinical practice guidelines.

To promote the implementation of EBP within the hospital, the educational department plans to offer an outreach training course based on an e-learning module in which the lessons learned are applied.

To disseminate the results outside the hospital, two symposia about EBP were organized, presenting the lessons learned in oral presentations and interactive workshops. 


\section{References}

1. EP-Nuffic. Het Amerikaanse onderwijssysteem beschreven en vergeleken met het Nederlandse [Comparison between the American and Dutch education systems]. The Hague. Retrieved from: https://www.epnuffic.nl/publicaties/vind-een-publicatie/onderwijssysteem-verenigde-staten.pdf. 2016

2. Brancheorganisaties zorg, NU'91, Landelijk Overleg Opleidingen Verpleegkunde, MBO raad \& V\&VN. Toekomstbestendige beroepen in de verpleging en verzorging [Future-proof professions in nursing]. Retrieved from: http://www.venvn.nl/Portals/1/Nieuws/2016\%20Documenten/ 20160113\%20Rapport\%20Toekomstbestendige\%20beroepen.pdf. 2015

3. Grol R, Wensing M, Eccles M, Davis D. Improving Patient Care: The Implementation of Change in Health Care. West Sussex: John Wiley \& Sons, Ltd. 2013.

4. Melnyk BM, Fineout-Overholt E, Mays MZ. The evidence-based practice beliefs and implementation scales: Psychometric properties of two new instruments. Worldviews Evid Based Nurs. 2008;5(4):208-216.

5. Vermeulen H, Tellingen van I, Maaskant J, Simons R. (2009). Verpleegkundige dossierbespreking: Een goed idee voor implementatie van EBP? [The patient case discussion for nurses: A good idea for implementation of EBP?] Nederlands Tijdschrift voor Evidence Based Practice. 2009;7(4):4-7.

6. Straus SE, Green ML. Evaluating the teaching of evidence based medicine: Conceptual framework. BMJ. 2004;329:1029-1032.

7. Sackett DL, Rosenberg WM, Gray JA, Haynes RB, Richardson WS. Evidence based medicine: What it is and what it isn't. BMJ. 1996;312(7023):71-72.

8. Sackett DL, Straus SE, Richardson WS, Rosenberg WM, Haynes RB. Evidence-based medicine: How to practice and teach EBM (2nd ed.). Edinburgh: Churchill Livingstone. 2000.

9. Holly C, Percy M, Caldwell B, Echevarria M, Bugel MJ, Salmond S. Moving evidence to practice: Reflections on a multisite academic-practice partnership. Int J Evid Based Healthc. 2014;12(1):31-38.

10. Melnyk BM, Fineout-Overholt E. Evidence-Based Practice in Nursing \& Healthcare. Philadelphia, PA: Wolters Kluwer Lippincott Williams \& Wilkins. 2014.

11. Goossens A, Vermeulen H, Ubbink D. Evidence-based richtlijnen [Evidence-Based Guidelines] (deel 3). Nederlands Tijdschrift Voor Evidence Based Practice. 2008;6(2):18-23.

12. Leeflang M, Hooft L, Vermeulen H. Diagnostisch onderzoek (deel 4). [Diagnostic Research] Nederlands Tijdschrift Voor Evidence Based Practice. 2008;6(3):18-23.

13. Ubbink D, Vermeulen H. Gerandomiseerd klinisch onderzoek (deel 1). [Randomized Clinical Trials] Nederlands Tijdschrift Voor Evidence Based Practice. 2007;5(6).

14. Vermeulen H, Korevaar J, Ubbink D. Patiënt-controle-onderzoek (deel 6). [Patient-control research design] Nederlands Tijdschrift Voor Evidence Based Practice. 2008;6(5):18-23.

15. Vermeulen H, Ubbink D. Cohort-onderzoek (deel 7). [Cohort research design] Nederlands Tijdschrift Voor Evidence Based Practice. 2009;7(1):7-11.

16. Vermeulen $H$, Ubbink D, Hooft L. Systematisch literatuuronderzoek van gerandomiseerde onderzoeken (deel 2) [Systematic literature searches of randomised studies (part 2)]. Nederlands Tijdschrift Voor Evidence Based Practice. 2008;6(1):4-8.

17. Vermeulen $H$, Ubbink D, Hooft L. Systematisch review van diagnostisch onderzoek (deel 5) [Systematic reviews of diagnostic studies (part 5)]. Nederlands Tijdschrift Voor Evidence Based Practice. 2008;6(4): 18-22.

18. Brouwers MC. Appraisal of Guidelines for Research \& Evaluation Instrument II. Canada: The Agree Next Steps Consortium. 2009.

19. Straus SE, Richardson WS, Glasziou P, Haynes RB. Evidence-based medicine: how to practice and teach EBM (2nd ed.). Edinburgh: Churchill Livingstone. 2010.

20. McCormack HM, Horne DJ, Sheather S. Clinical applications of visual analogue scales: A critical review. Psychol Med. 1988;18(4):1007-1019.

21. Dutch Ministry of Health. VMS Care: Early detection and treatment of pain. The Hague: VWS. Retrieved from: http://www.vmszorg.nl/_library/5544/web_2009.0109_praktijkgids_pijn.pdf. 2009.

22. Saunders RP, Evans MH, Joshi P. Developing a Process-Evaluation Plan for Assessing Health Promotion Program Implementation: A How-To Guide. Health Promot Pract. 2005;6(2):134-147. 
23. Melnyk BM, Fineout-Overholt E, Stillwell SB, Williamson KM. Evidence-based practice: Step by step: Igniting a spirit of inquiry: An essential foundation for evidence-based practice. Am J Nurs. 2009;109(11): 49-52.

24. Saunders $\mathrm{H}$, Vehvilainen-Julkunen $\mathrm{K}$. The state of readiness for evidence-based practice among nurses: An integrative review. Int J Nurs Stud. 2016;56:128-140. 




\section{Chapter 4}

Shared decision making in chronic care in the context of evidence-based practice in nursing:

a discussion

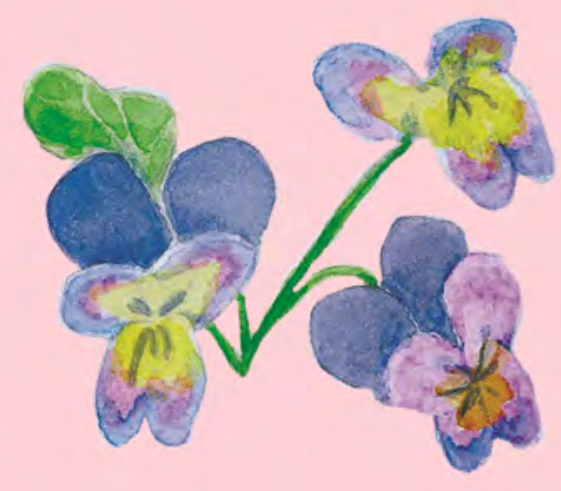

Jolanda H.H.M. Friesen-Storms

Gerrie J.J.W. Bours

Trudy van der Weijden

Anna J.H.M. Beurskens

International Journal of Nursing Studies 2015;52:393-402 


\section{Abstract}

In the decision-making environment of evidence-based practice, the following three sources of information must be integrated: research evidence of the intervention, clinical expertise, and the patient's values. In reality, evidence-based practice usually focuses on research evidence (which may be translated into clinical practice guidelines) and clinical expertise without considering the individual patient's values. The shared decision-making model seems to be helpful in the integration of the individual patient's values in evidence-based practice. We aim to discuss the relevance of shared decision making in chronic care and to suggest how it can be integrated with evidence-based practice in nursing.

We start by describing the following three possible approaches to guide the decision-making process: the paternalistic approach, the informed approach, and the shared decision-making approach. Implementation of shared decision making has gained considerable interest in cases lacking a strong best-treatment recommendation, and when the available treatment options are equivalent to some extent. We discuss that in chronic care it is important to always invite the patient to participate in the decision-making process.

We delineate the following six attributes of health care interventions in chronic care that influence the degree of shared decision making: the level of research evidence, the number of available intervention options, the burden of side effects, the impact on lifestyle, the patient group values, and the impact on resources.

Furthermore, the patient's willingness to participate in shared decision making, the clinical expertise of the nurse, and the context in which the decision making takes place affect the shared decision-making process. A knowledgeable and skilled nurse with a positive attitude towards shared decision making - integrated with evidence-based practice - can facilitate the shared decision-making process. We conclude that nurses as well as other health care professionals in chronic care should integrate shared decision making with evidence-based practice to deliver patient-centred care.

\section{What is already known about the topic?}

- Evidence-based practice advocates the integration of research evidence with clinical expertise and patient values in the decision-making process for an individual patient.

- $\quad$ The current focus in evidence-based practice - whether in education or research - is often limited to the use of research evidence.

- Shared decision making integrates research evidence with clinical expertise and patient values.

\section{What this paper adds}

- A discussion of the relevance of the integration of shared decision making to evidence-based practice in nursing for individual patients with a chronic condition.

- The identification of attributes of health care interventions, such as side effects or lifestyle impact, that are relevant to the integration of shared decision making with evidence-based practice.

- An illustration of how to integrate shared decision making with evidence-based practice in chronic care. 


\section{Introduction}

Evidence-based practice is widely recognised as being relevant to the improvement of nursing care. ${ }^{1-3}$ Its origin is in medical science (evidence-based medicine). Although there are several definitions of evidence-based practice, the most common definition is "the integration of the best research evidence with clinical expertise and the patient's preferences and values". ${ }^{4}$ Given the popularity of evidence-based medicine, the phrase has been radically expanded, adopted, and adapted by other disciplines within the health care arena. The nursing discipline adopted the term evidence-based nursing. ${ }^{5}$ Evidence-based practice has become a generic term used across various health care professions, including nursing.

Several variations of evidence-based practice models have been reported. Most of them share the following five process steps: (1) formulate answerable questions, (2) efficiently search for the best research evidence, (3) appraise the research evidence critically, (4) integrate the research evidence, clinical expertise, and the patient's values into the individual decision-making process, and (5) evaluate the process and result. ${ }^{6,7}$

Health care interventions using evidence-based practice are often determined by the level of the underlying research evidence, which is usually depicted in a hierarchal pyramid. Systematic reviews of randomised controlled trials and high-quality single randomised controlled trials are at the top of the hierarchy of research evidence, while editorials and expert opinions are at the bottom of that hierarchy. ${ }^{8}$ Nevertheless, the level of research evidence on efficacy often cannot be directly applied in clinical practice. Clinical guidelines have been developed to facilitate the interpretation of research evidence into clinical practice. GRADE (Grades of Recommendation, Assessment, Development, and Evaluation) ${ }^{9}$ offers a system for rating the strength of recommendations in clinical practice guidelines. The system is designed for guidelines that examine alternative management strategies or interventions, which may include no intervention or the current best management practices. ${ }^{10}$ GRADE provides a framework that, in addition to the quality of research evidence, takes into consideration the balance between the desirable and undesirable outcomes of alternative intervention strategies, patient group values, and resource use. These recommendations may vary in strength due to fluctuations in upgrading or downgrading the quality of evidence, the balance between desirable and undesirable outcomes (of alternative intervention strategies), patient values, or costs on the process. ${ }^{10}$

Education and research related to evidence-based practice emphasise the use of research evidence in clinical practice, ${ }^{11,12}$ which is sometimes translated into clinical practice guidelines, ${ }^{13}$ and focus less on the individual patient's values in the decisionmaking process. ${ }^{11,14,15}$ Counselling and educating patients about health care decisions fall 
within nurses' scope of practice, putting them in a position to influence the patient's choices. ${ }^{16}$ To help patients make a choice, nurses have to not only use research evidence but also interpret the evidence or recommendation to meet the needs of their individual patients in the decision-making process. ${ }^{11,15}$ See Box 4.1 for a case description. This skill requires integrating the research evidence or the clinical practice recommendation with clinical expertise while also integrating the individual patient's values into the process. $^{11,15}$

Box 4.1 Case description in which an evidence-based decision has to be made.

\section{Case 1}

A patient who is seriously ill and hospitalized is at risk for pressure ulcers. According to the latest research evidence, this patient has to lie down on a pressure ulcer prevention mattress and has to be repositioned on different sides every few hours. The nurse acts in accordance with this research evidence to prevent pressure ulcers. After a few weeks, however, this patient becomes pre-terminally ill and does not want to be repositioned anymore. The nurse now has to decide with the patient (and proxy) to comply with the research evidence on pressure ulcers or to comply with the patient's desire for more comfort. ${ }^{17,18}$

We agree with Satterfield et al., ${ }^{15}$ who acknowledge the importance of integrating shared decision making with evidence-based practice because shared decision making includes the individual patient's values into the process. We focus on integrating shared decision making with evidence-based practice for patients with a chronic condition in nursing.

Shared decision making is a process that aims to have the health care professional and the patient jointly arrive at a health care choice that is based on the best available research evidence, clinical expertise, and the values of the informed patient. This is the crux of patient-centred care. ${ }^{19-21}$ The rationale for shared decision making is based on research evidence that patients want to participate more in their own health care decisions $^{14,22,23}$ and on the ethical principle of patient autonomy. ${ }^{24}$ The rationale for evidence-based practice is based on the claim that an intervention needs to be justified based on research evidence of the effectiveness of the intervention ${ }^{24}$ because this leads to a better standard of care. ${ }^{25}$ Evidence-based practice, which currently emphasises research evidence, is sometimes described in the literature as being inimical to patientcentred care. ${ }^{25}$ In contrast, shared decision making has been acknowledged by some authors $^{14,26}$ as having the potential to strengthen the decision-making process in evidence-based practice by integrating the research evidence with clinical expertise and the individual patient's values. However, how this can be accomplished is still unclear. ${ }^{14}$ In nursing, shared decision making seems particularly relevant in chronic care because it may enhance self-management, self-efficacy, and patient empowerment. ${ }^{27,28}$ In chronic $^{2}$ 
care, self-management is inescapable because patients are faced with decisions that influence the course of their disease on a daily basis. ${ }^{29}$ Self-efficacy, defined as the confidence to carry out certain behaviours in order to reach a specific goal, is recognised as a prerequisite for effective self-management in chronic diseases. ${ }^{29,30}$ We define chronic care, in accordance with 'The Expanded Chronic Care Model', ${ }^{31}$ as the prevention and management of a chronic disease that is provided by interdisciplinary and inclusive teams in the health care system and the community. Nurses have long been recognised as having a key role in interdisciplinary teams in helping patients manage their chronic disease(s). ${ }^{32-34}$

\section{Purpose}

This paper aims to discuss the relevance of shared decision making in chronic care and to suggest how it can be integrated with evidence-based practice in nursing. The discussion is primarily relevant to countries in which advance practice nursing and teambased practice is within nursing's scope of practice. However, the discussion is also applicable to other countries.

The discussion primarily elaborates on Step 4 of evidence-based practice (integrating research evidence, clinical expertise, and patient's values into decision-making). The paper first explains shared decision making as one of several possible approaches to guide the decision-making process. It then discusses the integration of shared decision making with evidence-based chronic care, including a description of the different attributes of interventions in health care that determine the degree of shared decision making. The paper continues with a discussion on the impact of the patient, the nurse, and the context on shared decision making. Finally, the implications for nursing are addressed.

\section{Methodology}

It is not the intention of this paper to provide a systematic and complete review of the literature, but the CINAHL and PubMed digital databases were searched, and a network of experts on evidence-based practice and shared decision making was consulted to include the most relevant papers that underpin our discussion based on previous literature and to ensure that our key points had not already been published elsewhere. The literature search strategy used key words such as '(shared) decision making', 
'evidence-based', and 'nurs*' in various combinations. Search parameters were limited to English, Dutch, and German language texts with no date restrictions.

Articles dated through October 2013 in all of the databases were included if they reflected on shared decision making or interventions to implement shared decision making.

\section{Shared decision making as an approach to guide the decision- making process}

Although health care professionals are legally obligated to inform clients about their treatment and are required to ask for informed consent, ${ }^{35}$ there are no legal restrictions regulating the manner in which this is carried out.

Table 4.1 describes three possible approaches to guide the decision-making process. ${ }^{36}$ The paternalistic approach involves the health care professional deciding - either independently or with other health care professionals - what the best treatment or intervention for the patient would be and subsequently informing the patient of that decision and convincing the patient to follow through with that choice. On the other end of the spectrum is the informed approach, in which the patient is provided with information and is entitled to make the decision. Shared decision making is an approach somewhere between these two extremes. Expert information is not only shared from health care professionals to the patient (and proxy) but the patient also shares personal information (patient values) with the health care professional. When the health care professional and the patient deliberate on screening, diagnostic, therapeutic, or palliative interventions, ${ }^{37}$ the decision-making process is a shared experience. ${ }^{36}$

Table 4.1 Analytical stages of treatment decision making in different approaches. ${ }^{36}$

\begin{tabular}{|c|c|c|c|}
\hline & Paternalistic approach & Shared approach & Informed patient approach \\
\hline $\begin{array}{l}\text { Information } \\
\text { exchanges }\end{array}$ & $\begin{array}{l}\text { Medical, legally required } \\
\text { one-way transfer of } \\
\text { minimum information from } \\
\text { health care professional to } \\
\text { patient }\end{array}$ & $\begin{array}{l}\text { Medical and personal } \\
\text { information relevant for } \\
\text { decision making is exchanged } \\
\text { between patient and health } \\
\text { care professionals }\end{array}$ & $\begin{array}{l}\text { All relevant information } \\
\text { shared largely one way } \\
\text { from health care } \\
\text { professional to patient }\end{array}$ \\
\hline Deliberation & $\begin{array}{l}\text { Health care professional } \\
\text { (plus potential other } \\
\text { professionals) }\end{array}$ & $\begin{array}{l}\text { Health care professional and } \\
\text { patient (plus potential others) }\end{array}$ & $\begin{array}{l}\text { Patient (plus potential } \\
\text { others) }\end{array}$ \\
\hline $\begin{array}{l}\text { Deciding on } \\
\text { treatment to } \\
\text { implement }\end{array}$ & Health care professional & $\begin{array}{l}\text { Shared between health care } \\
\text { professionals and patient }\end{array}$ & Patient \\
\hline
\end{tabular}


Since the early 1990s, shared decision making has gained considerable interest in optionsensitive or preference-sensitive decisions regarding screening and treatment options, which might also include a watchful waiting strategy. These types of decisions are considered option-sensitive or preference-sensitive because there is insufficient evidence about outcomes or there is a need to trade-off known benefits or harms between competing options. ${ }^{38,39}$ An example of this would be the choice between surgery and radiation in treating cancer when both options have competing benefits or harm. $^{39}$

Various models have been developed to demonstrate how shared decision making can be applied in the clinical setting. ${ }^{40}$ The essential elements of shared decision making ${ }^{40}$ are as follows: define and explain the problem, present options, discuss the pros and cons, clarify the patient's values and preferences, discuss the patient's ability and selfefficacy, discuss the health care professional's knowledge and recommendations, check and clarify the patient's understanding, make or explicitly defer the decision, and arrange follow up. The following additional elements are considered ideal: provision of unbiased information, definition of the patient's desired role of involvement, presentation of evidence including probabilities of treatment outcomes, and mutual agreement. $^{40,41}$ However, a complete consensus on the concept has not yet been reached. $^{40}$

A well-known model of shared decision making created by nurses is "The Ottawa Decision Support Framework". ${ }^{41,42}$ The framework uses a three-step process that assesses the patient and practitioner determinants of decisions to identify decision support needs, provides decision support tailored to the patient's needs, and evaluates the decision-making process and outcomes. "The Ottawa Decision Support Framework" has been extensively validated through decisional needs assessment studies with patients, the public, and healthcare professionals. ${ }^{43}$

Recently, Elwyn et al. ${ }^{44}$ developed a simplified shared decision-making model in light of applicability for clinical practice. The essential elements of the model include choice talk, option talk, and decision talk (see Table 4.2). Choice talk in this model is about clarifying that reasonable options are available to patients. Option talk refers to providing detailed information about the pros and cons of each option, and decision talk refers to supporting the process of considering the patient's preferences (including the patient's right to opt out of making a decision) and deciding on the best option; it is the deliberation. This model emphasises the importance of good communication skills. ${ }^{44}$

To support shared decision making and integrate it in clinical practice, decision aids have been developed, evaluated, and implemented. Patient decision support tools or decision aids are decision support interventions that help people make choices by describing why 
and where choices exist and by providing information about the possible consequences of choices. ${ }^{45}$ Their format can be very diverse, ranging from printed fact sheets and booklets to CD-ROMs, videos and interactive websites. ${ }^{46}$

Table 4.2 Key elements of shared decision making. ${ }^{44}$

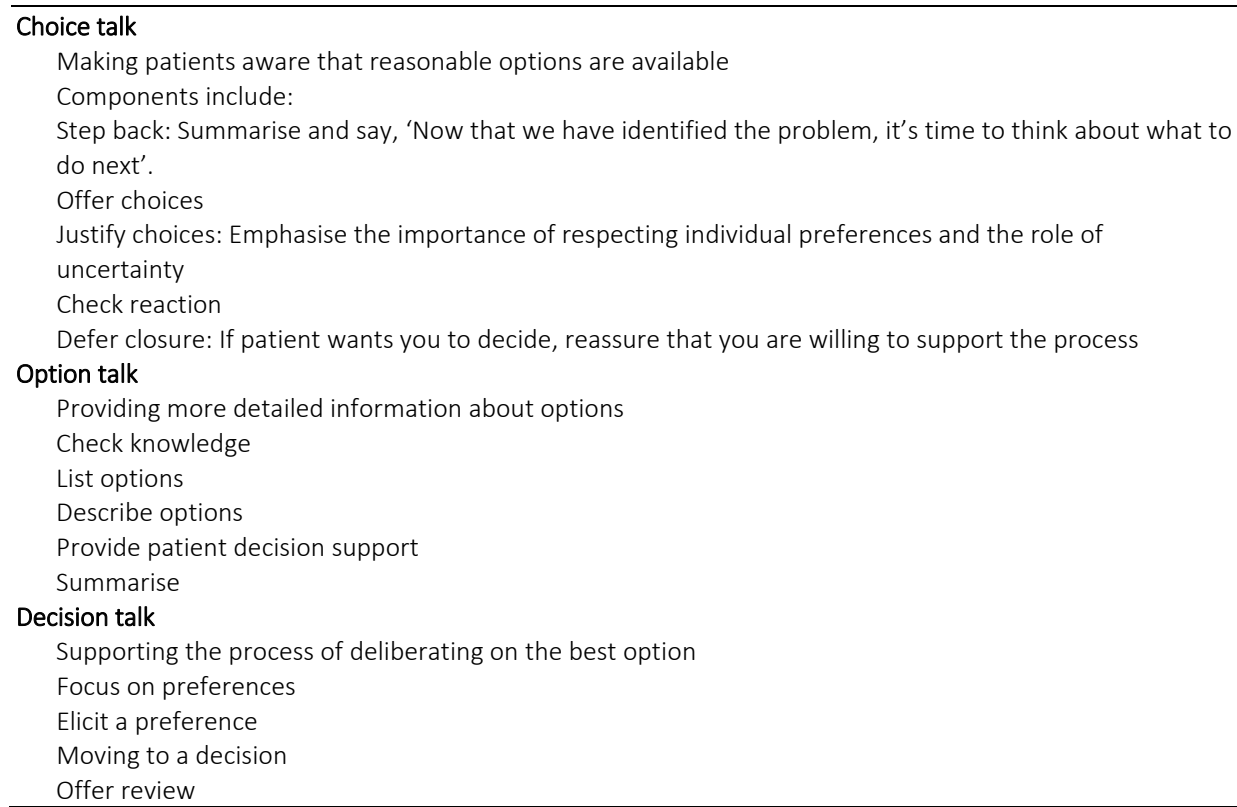

Exposure to patient decision support tools compared to usual care results in increased knowledge of patients, lower decisional conflict, reduced proportions of people who are passive in decision making and reduced proportions of people who remain undecided post-intervention. ${ }^{39}$

\section{Shared decision making in evidence-based chronic care in nursing}

The nurse (or other health care professional) may opt for an approach more like the paternalistic approach when the level of research evidence is high or a recommendation in a clinical guideline is strong. An example of this would be the high level of research evidence and strong recommendation for the beneficial effect of a daily low-dose of aspirin for people who have a high risk of heart attack. ${ }^{47}$ 
Shared decision making is often seen, as described in Section 4, as a model that should be used when there is no clear research evidence on the best treatment decision or screening decision and when the options available are, to some extent, equivalent. In chronic care, however, even when the level of research evidence for an option is high and no other competing options exist, there are attributes of the intervention that may require additional considerations. An example of this might be when an intervention dramatically interferes with the patient's lifestyle such that a paternalistic approach is inappropriate. The informed patient approach provides the patient with information and leaves the decision to the patient; however, many patients find it difficult to make the decision themselves. This approach may create uncertainty, and patients may feel abandoned. ${ }^{44,48}$ However, they do feel supported when deliberating and sharing in the decision-making process with an empathic professional. Therefore, we advocate inviting the chronic patient to be involved in the decision-making processes for their own health care, which in turn may stimulate self-efficacy and self-management. ${ }^{27,28}$

Shared decision making can be integrated with the decision-making step of evidencebased practice in chronic care as depicted in the graph in Figure 4.1. Although we believe that in chronic care it is important to always invite the patient to participate in the decision-making process, in our opinion, the degree of shared decision making depends on different attributes of the health care intervention. We delineate five attributes based on the four determinants of the GRADE system ${ }^{10}$ that influence the degree of shared decision making in chronic care. Those attributes are the level of research evidence (quality of research evidence), the presence of intervention options (reasonable alternative management strategies or interventions), the burden of side effects (undesirable consequences), patient group values and the impact on resources (resource use) (see Figure 4.1). We added the impact on lifestyle as a sixth attribute. The undesirable consequences $^{10,49}$ implicitly include impact on lifestyle, for example, the inconvenience of a treatment option. We added this as an additional attribute because lifestyle behaviours are important factors in preventing chronic diseases, and adapting one's lifestyle to a chronic disease is not easy. ${ }^{50}$

The degree of shared decision making may be lower when the level of research evidence is high, only one option is available, there is no burden of side effects, there is little impact on lifestyle, there is evidence that patients generally consent to the intervention, and there is little impact on resources (see left side of Figure 4.1). In contrast, the degree of shared decision making is high when the level of research evidence is low, more options are available, the burden of side effects is high, the impact on life style is high, there is evidence that patients vary considerably in their values towards the intervention, and the impact on resources is high (see right side of Figure 4.1). In 
between these conditions, various combinations of attributes are possible, leading to different degrees of shared decision making.
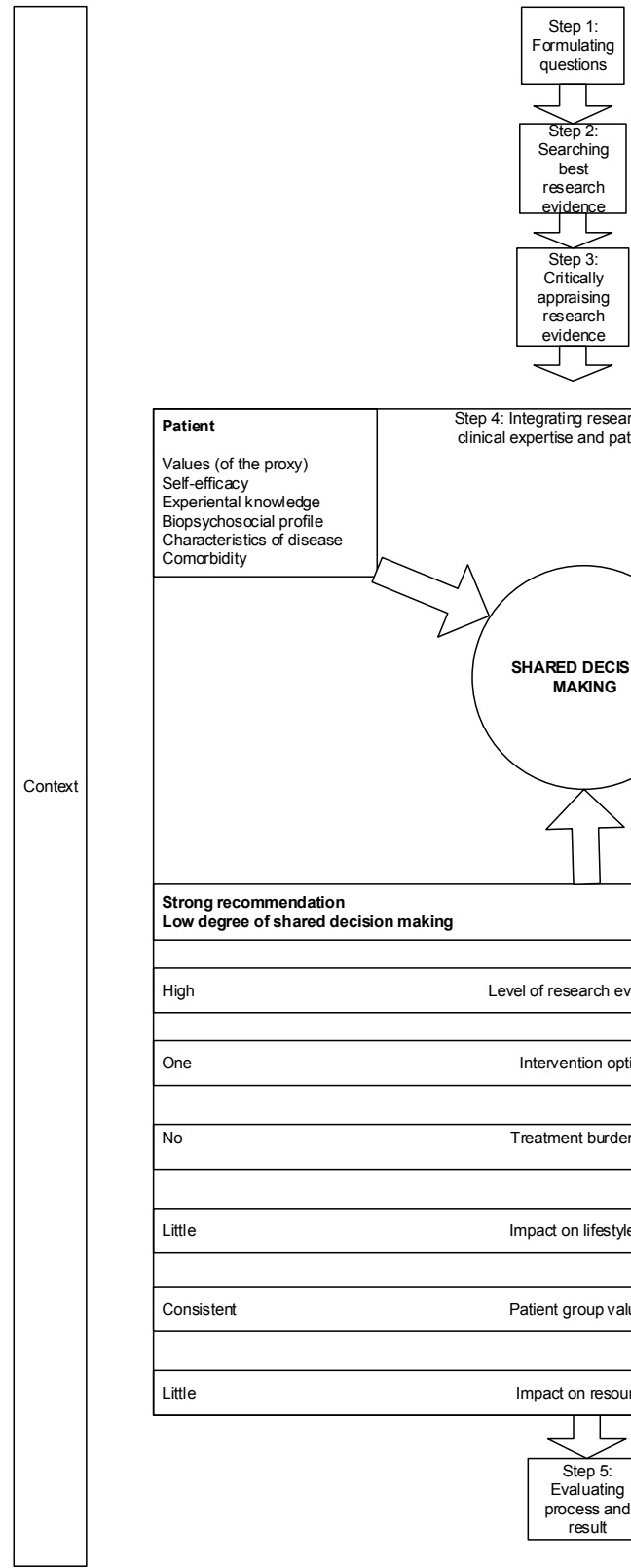

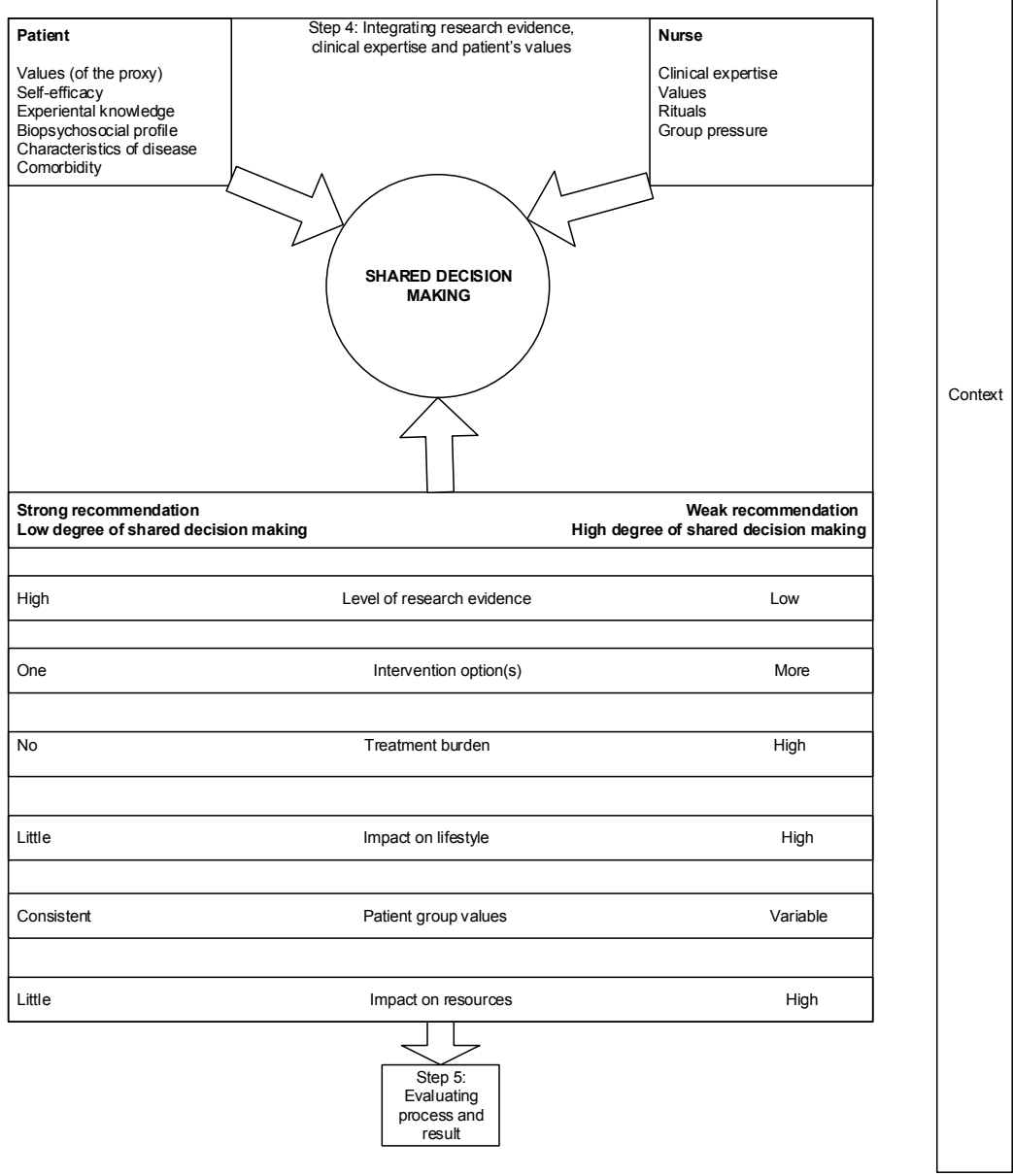

Figure 4.1 Integrating shared decision making with evidence-based practice. 
Of course the patient always has a choice, even when there is one strong option with no competing options, to not follow a recommendation. The two patient cases described in Box 4.2 illustrate different individual decisions based on these attributes.

Box 4.2 Two case descriptions of shared decision making.

Example 1: One option, a high level of research evidence, a high burden of side effects, a high impact on lifestyle, little impact on resources, a low self-efficacy patient

A female patient with complicated type 2 diabetes who uses insulin twice a day has highly fluctuating blood glucose levels. She has been struggling with obesity since childhood. She works as an accountant and is not physically active. She does not like to exercise. Research evidence indicates that physical activity and weight loss are important lifestyle interventions for this patient due to their beneficial effects on carbohydrate metabolism, insulin sensitivity, and prevention of long-term complications. ${ }^{51-53}$

Choice talk: The patient and nurse can define the problem of diabetes mellitus that is not well managed, obesity, physical inactivity, and their effects on diabetes management.

Option talk: Information can be exchanged; the nurse can inform the patient about the research evidence of weight loss and exercise in diabetes. Side effects and the patient's values, resources, lifestyle and selfefficacy can be explored and discussed. Support in exploring different methods of losing weight and ways to exercise more can be provided. Deliberation about option(s) is strong.

Decision talk: Sharing ideas about and supporting the patient in deciding on how to reach possible weight loss and more physical activity, taking management of side effects, patient values, lifestyle, and self-efficacy into consideration.

Example 2: More intervention options, conflicting research evidence, different side effects, different impacts on lifestyle, different impacts on resources, a low self-efficacy patient

A female patient with type 2 diabetes is using tablets to control her diabetes. Her blood glucose levels and $\mathrm{HbA}_{1 \mathrm{c}}{ }^{1}$ are not well controlled. She has been struggling with obesity since childhood. She works as an accountant and is not physically active. She does not like to exercise. The diabetic nurse talked with her several times about switching from tablets to insulin, but this is a big step for the patient. She is afraid of needles.

Choice talk: The patient and nurse can define the problem of blood glucose levels and HbA1c that are not well controlled and that may lead to more short- and long-term complications. A different treatment for diabetes is needed. For this patient, there are two options: weight loss with more physical activity or a switch from tablets to insulin (possibly combined with weight loss and more physical activity).

Option talk: Information can be exchanged. The nurse can inform the patient about the two options and the side effects in more detail. Patient values, lifestyle, and self-efficacy of both options can be explored and discussed. Deliberation about options is strong.

Decision talk: The patient is supported in her exploration of what the best option for her is and ideas are exchanged on how to reach this option while taking the management of side effects, patient values, lifestyle, and self-efficacy into consideration.

${ }^{1}$ HbA1c: designates a stable minor glycated sub-fraction of haemoglobin. It is a reflection of the mean blood glucose levels during the most recent 6-8 weeks and is expressed in percentage of total haemoglobin.

To facilitate shared decision making in clinical practice (as stated in Section 4), Elwyn et al. ${ }^{44}$ designed a feasible three-step model to interpret conceptual descriptions of shared 
decision making into routine practice. Although this model is built around having alternative preference-sensitive options, we think it could also be used in chronic care to discuss the influence of the attributes when there is only one strong option available. The choice talk in this case might define the problem and offer the patient a choice in doing something about the problem. The option talk step provides information on research evidence, side effects, impact on lifestyle, and impact on resources. It also serves to begin an exploratory discussion of the patient's values and the patient's selfefficacy with regard to an option, and it provides support to explore different ways of adapting this option to the patient's lifestyle. The decision talk might involve sharing ideas and supporting the patient in deciding how this option can be adapted to the patient's lifestyle.

\section{Patient, nurse and context influence the decision-making process}

\section{Patient}

Shared decision making requires the patient's willingness to participate in the decisionmaking process. Nevertheless, even after they have been informed of the range of available options, patients may still prefer to leave the decision to the health care professionals. ${ }^{27}$ These people wilfully forfeit some of their decision-making power to the health care professionals, which Moser et al. ${ }^{54}$ call 'welcomed paternalism'.

The willingness of the patient to participate in shared decision making is affected by a few factors. Patients are more likely to prefer shared decision making after the acute stage of a disease ${ }^{27,55}$ rather than during it. In acute care, as with first aid situations, for example, there is often not enough time or need to use shared decision making. However, the care of chronic diseases is different because no immediate life-threatening situation exists. Age also seems to influence the willingness to participate in shared decision making. Younger patients regard shared decision making as the preferred style of interaction with health care professionals more frequently than older patients. ${ }^{27,56,57}$ Furthermore, patients with higher levels of education seem to prefer shared decision making more often than patients with a lower educational level. ${ }^{27,56,57}$

Although the expressed preferences of patients to be involved in decision making differ, research indicates that patients want more involvement in decision making compared to what actually occurs. ${ }^{58}$ In a study of preferred and actual roles in decision making for cancer patients, Singh et al. ${ }^{57}$ reported that 40 per cent of the patients experienced discordance. Therefore, it is important for nurses to explore the patient's desire to be 
involved in the decision-making process. However, nurses have to bear in mind that when offered a role in a decision-making process, some patients initially decline decisional responsibility because they are surprised, unsettled, or uncertain. ${ }^{44}$ They want health care professionals to tell them what to do. If this occurs in a patient consultation, Elwyn et al. ${ }^{44}$ suggest 'deferring closure' by reassuring the patient that the health care professionals are willing to support the process and are not abandoning the patient. If a patient who has been informed on the decisional dilemma ultimately does not want to engage in the shared process, this decision also has to be respected.

\section{Nurse}

The nurse's clinical expertise, mode of professional preparation, depth of professional knowledge, skills, attitude, and values influence the decision-making process. ${ }^{59-61}$ Clinical expertise is expressed and embedded in daily practice, and it is often tacit and intuitive. ${ }^{60}$ Furthermore, nurses not only act on their own clinical expertise but also on the clinical expertise of other nurses and health care professionals. ${ }^{61}$ Moral norm, defined as the person's feeling of moral obligation towards performing a given behaviour, was identified by Cote et al. ${ }^{62}$ as the most important predictor of nurses' intention to use research evidence in decision making. A nurse who has knowledge, skills, and a positive attitude towards shared decision making and evidence-based practice is in the position to facilitate a shared decision-making process. ${ }^{3,63}$

\section{Context}

There are many rituals or routines in the nursing profession that are often the result of social influences within a group (peer pressure). These can potentially hinder the integration of shared decision making with evidence-based practice. ${ }^{64}$ Other contextual factors that influence decision making include the following:

- Social factors such as local opinion leaders in nursing teams and the nursing culture.

- Organisational factors such as time for research activities, hospital size, staffing support, organisational innovativeness, integration of recommendations into organisational structures and processes, access to resources and research findings, organisational climate, provision of education, availability of knowledge and skills within organisations, inter-organisational collaboration, finances, and workload.

- Societal factors such as crises in nursing or workforce shortages. ${ }^{65}$ 
Edwards and Elwyn ${ }^{63}$ mentioned the following requirements for an organisation that supports shared decision making:

- A culture of participation and patient-centeredness in which patient involvement in treatment options is a key outcome.

- Agreement about situations in which shared decision making is needed, initiated, accessed, and sustained;

- Inter-professional collaboration with formal and informal communication routines;

- Data systems such as digital patient records;

- Continuing professional development;

- Decision support systems and technologies.

\section{Implications for nursing}

After identifying three ways of decision making, it is our opinion that chronic care patients should be offered shared decision making. However, as discussed above, the degree of shared decision making may differ based on different attributes of health care interventions. The patient, the nurse, and the context of the situation also influence this process; all have several implications for nursing.

For the nurse, a positive attitude towards shared decision making and evidence-based practice is required to integrate the two., ${ }^{3,66,67}$ Nurses have to acknowledge the importance of exploring the wishes of the patient to be involved in a decision-making process while also keeping in mind that patients may initially decline decisional responsibility and that they may change their minds once they are informed of the range of options available to them.

The essential skills for establishing effective shared decision making are good clinical communication skills that include developing a rapport with the patient, providing focused communication and risk communication, and having the ability to explore the patient's values and preferences. ${ }^{28,38,44}$ Evidence-based practice skills are also relevant to the process. ${ }^{63}$

As reported in the literature, nurses do not always possess sufficient communication or evidence-based practice skills, and their attitude towards shared decision making and evidence-based practice is not always positive. ${ }^{27,68,69}$ Providing nurses with training in shared decision making and helping them acquire a patient-centred attitude, along with the implementation of decision support tools, could significantly improve nurses' use of shared decision making. ${ }^{20}$ The implementation of decision support tools is important because they are effective in helping patients make choices. ${ }^{39}$ 
The importance of considering and addressing contextual factors in establishing shared decision making and evidence-based practice is recognised. ${ }^{59,63}$ For instance, it is important that nurses become aware of existing rituals and routines and develop a critical attitude towards adhering to these rituals. Furthermore, establishing a closer link between clinical practice guidelines and decision support tools that provide information about the six attributes of health care interventions might help nurses establish a more effective use of shared decision making and evidence-based practice. ${ }^{45}$

\section{Implications for future research}

Several research questions on the implementation of shared decision making in chronic care in nursing emerge from this discussion. Patients as well as nurses need to be empowered in the shared decision-making process. Furthermore, for nurses as well as other health care professionals, the ability to recognise a patient's willingness to participate soundly in shared decision making is invaluable. The question of how to accomplish this -through education, training, instruction- then arises. In this context, patient decision-making tools are significant. The diversity of their format raises the question as to what their preferred format should be. Research on valid decision-making instruments is recommended.

Although the discussion addresses the relevance of shared decision making in evidencebased practice in chronic care, the preferred timing and integration of shared decision making in the patient-care chain and the optimal usage of shared decision making are questions to be addressed in future research. Finally, the importance of establishing a closer link between clinical practice guidelines and decision support tools is recognised. However, the question of how clinical practice guidelines can be adapted to facilitate shared decision making remains.

\section{Conclusion}

The literature seems to regard shared decision making and evidence-based practice as two different concepts and processes. However, shared decision making acknowledges the importance of evidence-based practice, and evidence-based practice acknowledges the importance of integrating patient values and clinical expertise with research evidence. This article addresses the apparent suitability of shared decision making as a model for many chronic care decisions that comprise different attributes of health care 
interventions, the patient's values, the nurse, and the context that affect shared decision making when integrated with evidence-based practice.

Establishing a policy of shared decision making within an organisation is not easy. It requires a positive attitude and nurses with specific communication skills, such as risk communication and value elicitation. Providing nurses with proper training and the development of clinical guidelines and decision support tools for patients with established links to these guidelines are essential for the provision of the support that is crucial for proper implementation of shared decision making by nurses.

We believe that nurses, as well as other health care professionals in chronic care, should integrate shared decision making with evidence-based practice to deliver patientcentred care and to involve patients in their decision-making process, which in turn may encourage self-efficacy, self-management, and patient empowerment. 


\section{References}

1. Grimshaw J, Eccles M, Thomas R, MacLennan G, Ramsay C, Fraser C, Vale L. Toward evidence-based quality improvement: evidence (and its limitations) of the effectiveness of guideline dissemination and implementation Strategies 1966-1998. J Gen Intern Med. 2006;21:S14-20.

2. Heater BS, Becker AM, Olson RK. Nursing Interventions and Patient Outcomes: a meta-analysis of studies. Nursing Research. 1988;37(5):303-307.

3. Melnyk BM, Fineout-Overholt E. The evidence-based practice beliefs and implementation scales: psychometric properties of two new instruments. Worldviews Evid Based Nurs. 2008;5(4):208-216.

4. Sackett DL, Straus SE, Richardson WS, Rosenberg WM, Haynes RB. Evidence-Based Medicine: How to Practice and Teach EBM. Churchill Livingstone, Edinburgh. 2000.

5. French P. The development of EBN. J Adv Nurs. 1999;29:72-78.

6. Sackett DL, Rosenberg WMC. The need for evidence-based medicine. J R Soc Med 1995;88(11):620-624.

7. Gawlinski A, Rutledge D. 2008. Selecting a model for evidence-based practice changes: a practical approach. AACN Adv Crit Care. 2008; 19(3):291-300.

8. Mantzoukas S. A review of evidence-based practice, nursing research and reflection: levelling the hierarchy. J Clin Nurs. 2008;17(2):214-223.

9. Guyatt GH, Oxman AD, Kunz R, Falck-Ytter Y, Vist GE, Liberati A, Schunemann HJ. Going from evidence to recommendations. BMJ. 2008;336(7652):1049-1051.

10. Guyatt G, Oxman AD, Akl EA, Kunz R, Vist G, Brozek J, Norris S, Falck-Ytter Y, Glasziou P, DeBeer H, Jaeschke R, Rind D, Meerpohl J, Dahm P, Schunemann HJ. 2011. GRADE guidelines: 1. Introduction GRADE evidence profiles and summary of findings tables. J Clin Epidemiol. 2011;64(4):383-394.

11. Melnyk BM, Fineout-Overholt E. 2006. Consumer preferences and values as an integral key to evidencebased practice. Nurs Adm Q. 2006;30(2):123-127.

12. Squires J, Estabrooks C, Gustavsson P, Wallin L. Individual determinants of research utilization by nurses: a systematic review update. Implement Sci. 2011;6(1):1.

13. May C, Sibley A, Hunt K. 2014. The nursing work of hospital-based clinical practice guideline implementation: an explanatory systematic review using normalisation process theory. Int J Nurs Stud. 2014;51(2):289-299.

14. Barratt A. Evidence based medicine and shared decision making: the challenge of getting both evidence and preferences into health care. Patient Educ Couns. 2008;73:407-412.

15. Satterfield JM, Spring B, Brownson RC, Mullen EJ, Newhouse RP, Walker BB, Whitlock EP. Toward a transdisciplinary model of evidence-based practice. Milbank Q. 2009;87(2):368-390.

16. George TP. How nurses can encourage shared decision making. Nursing (Lond.). 2013;43(8):65-66.

17. European Pressure Ulcer Advisory Panel and National Pressure Ulcer Advisory Panel. Pressure Ulcer Treatment: Quick Reference Guide. National Pressure Ulcer Advisory Panel, Washington, DC. 2009.

18. European Pressure Ulcer Advisory Panel and National Pressure Ulcer Advisory Panel. Prevention of Pressure Ulcers: Quick Reference Guide. National Pressure Ulcer Advisory Panel, Washington, DC. 2009.

19. Charles C, Gafni A, Whelan T. 1997. Shared decision-making in the medical encounter: what does it mean? (or it takes at least two to tango). Soc Sci Med. 1997;44(5):681-692.

20. Légaré F, Ratté S, Stacey D, Kryworuchko J, Gravel K, Graham I, Turcotte S. Interventions for improving the adoption of shared decision making by healthcare professionals. Cochrane Database Syst Rev. 2010;(5):1-45.

21. Weston WW. Informed and shared decision-making: the crux of patient-centered care. CMAJ. 2001;165(4):438-439.

22. Coulter A. Partnerships with patients: the pros and cons of shared clinical decision-making. J Health Serv Res Policy 1997;2(2):112-121.

23. Coulter A. Paternalism or partnership? Patients have grown up-and there's no going back. BMJ. 1999;319(7212):719-720.

24. Parker M. The ethics of evidence-based patient choice. Health Expect. 2001;4(2):87-91.

25. Armstrong D. Clinical autonomy, individual and collective: the problem of changing doctors' behaviour. Soc Sci Med. 2002;55(10): 1771-1777. 
26. Vandvik PO, Brandt L, Alonso-Coello P, Treweek S, Akl EA, Kristiansen A, Fog-Heen A, Agoritsas T, Montori VM, Guyatt G. Creating clinical practice guidelines we can trust, use, and share: A new era is imminent. Chest. 2013;144(2):381-389.

27. Clark NM, Nelson BW, Valerio MA, Gong ZM, Taylor-Fishwick JC, Fletcher M. 2009. Consideration of shared decision making in nursing: a review of clinicians' perceptions and interventions. Open Nurs J. 2009;3:65-75.

28. Zoffmann V, Harder I, Kirkevold M. A person-centered communication and reflection model: sharing decision-making in chronic care. Qual Health Res. 2008;18(5):670-685.

29. Bodenheimer T, Lorig K, Holman H, Grumbach K. Patient self-management of chronic disease in primary care. JAMA. 2002;288(19):2469-2475.

30. Freund T, Gensichen J, Goetz K, Szecsenyi J, Mahler C. Evaluating self-efficacy for managing chronic disease: psychometric properties of the six-item self-efficacy scale in Germany. J Eval Clin Pract. 2013;19(1):39-43.

31. Barr VJ, Robinson S, Marin-Link B, Underhill L, Dotts A, Ravensdale D, Salivaras S. The expanded chronic care model: an integration of concepts and strategies from population health promotion and the chronic care model. Hosp Q. 2003;7(1):73-82.

32. Audit Commission. First Assessment: A Review of District Nursing Services in England and Wales. Audit Commission Publications, Abingdon. 1999.

33. Kendall S, Wilson P, Procter S, Brooks F, Bunn F, Gage H, McNeilly E. The Nursing Contribution to Chronic Disease Management: A Whole Systems Approach: Report for the National Institute for Health Research Service Delivery and Organisation programme. Queen's Printer and Controller of HMSO 2010, Herts. 2010.

34. Kratz CR. Care of the Long-Term Sick in the Community : Particularly Patients with Stroke. Churchill Livingstone, Edinburgh. 1978.

35. Presidential Commission on Medical Ethics. Making Health Care Decisions: The Ethical and Legal Implications of Informed Consent in the Patient-Practitioner Relationship. President's Commission, Washington, DC. 1982.

36. Charles C, Gafni A, Whelan T. Decision-making in the physician-patient encounter: revisiting the shared treatment decision-making model. Soc Sci Med. 1999;49(5):651-661.

37. Charles C, Whelan T, Gafni A, Farrell S. Shared Treatment Decision Making: what does it Mean to Physicians? J Clin Oncol. 2003;21(5):932-936.

38. Elwyn G, Edwards A, Kinnersley P, Grol R. 2000. Shared decision making and the concept of equipoise: the competences of involving patients in healthcare choices. Br J Gen Pract. 2000;50(460):892-899.

39. Stacey D, Legare F, Col NF, Bennett CL, Barry MJ, Eden KB, Holmes-Rovner M, Llewellyn-Thomas $H$, Lyddiatt A, Thomson R, Trevena L, Wu JHC. Decision aids for people facing health treatment or screening decisions. Cochrane Database Syst Rev. 2014;(1):1-331.

40. Makoul G, Clayman ML. An integrative model of shared decision making in medical encounters. Patient Educ Couns. 2006;60(3): 301-312.

41. O'Connor A, Stacey D, Jacobsen MJ. Ottowa Decision Support Tutorial: Improving Practitioners' Decision Support Skills. Ottowa Hospital Research Institute: Patient Decision Aids. Retrieved from: https://decisionaid.ohri.ca/ODST/pdfs/ODST.pdf. 2011. (Accessed 5 February 2014)

42. O'Connor AM, Tugwell P, Wells GA, Elmslie T, Jolly E, Hollingworth G, McPherson R, Bunn H, Graham I, Drake E. A decision aid for women considering hormone therapy after menopause: decision support framework and evaluation. Patient Educ Couns.1998;3 (3):267-279.

43. Ottowa Hospital Research Institute. Ottowa Decision Support Framework: Update, Gaps and Research Priorities. Ottowa Hospital Research Institute, Ottowa. Retrieved from: https://decisionaid.ohri.ca/docs/ODSF-workshop/ODSF-Workshop-Summary.pdf. 2010. (Accessed 5 February 2014)

44. Elwyn G, Frosch D, Thomson R, Joseph-Williams N, Lloyd A, Kinnersley P, Cording E, Tomson D, Dodd C, Rollnick S, Edwards A, Barry M. Shared decision making: a model for clinical practice. J Gen Intern Med. 2012;27(10):1361-1367.

45. van der Weijden T, Boivin A, Burgers J, Schünemann HJ, Elwyn G. Clinical practice guidelines and patient decision aids: an inevitable relationship. J Clin Epidemiol. 2012;65(6):584-589. 
46. Elwyn G, Frosch D, Volandes AE, Edwards A, Montori VM. Investing in deliberation: a definition and classification of decision support interventions for people facing difficult health decisions. Med Decis Making. 2010;30(6):701-711.

47. Fihn SD, Gardin JM, Abrams J, Berra K, Blankenship JC, Dallas AP, Douglas PS, Foody JM, Gerber TC, Hinderliter AL, King SB 3rd, Kligfield PD, Krumholz HM, Kwong RY, Lim MJ, Linderbaum JA, Mack MJ, Munger MA, Prager RL, Sabik JF, Shaw LJ, Sikkema JD, Smith CR Jr, Smith SC Jr, Spertus JA, Williams SV. 2012 ACCF/AHA/ACP/AATS/PCNA/SCAI/STS guideline for the diagnosis and management of patients with stable ischemic heart disease: Executive summary: A report of the American College of Cardiology Foundation/American Heart Association task force on practice guidelines, and the American College of Physicians, American Association for Thoracic Surgery, Preventive Cardiovascular Nurses Association, Society for Cardiovascular Angiography and Interventions, and Society of Thoracic Surgeons. Circulation. 2012;126(25);3097-3137.

48. Quill TE, Cassel CK. Nonabandonment: a central obligation for physicians. Ann Intern Med. 1995;122(5):368-374.

49. Andrews JC, Schunemann HJ, Oxman AD, Pottie K, Meerpohl JJ, Coello PA, Rind D, Montori VM, Brito JP, Norris S, Elbarbary M, Post P, Nasser M, Shukla V, Jaeschke R, Brozek J, Djulbegovic B, Guyatt G. GRADE guidelines: 15. Going from evidence to recommendation - determinants of a recommendation's direction and strength. J Clin Epidemiol.2013;66(7):726-735.

50. Wing RR, Goldstein MG, Acton KJ, Birch LL, Jakicic JM, Sallis JF Jr, Smith-West D, Jeffery RW, Surwit RS. Behavioral science research in diabetes: lifestyle changes related to obesity, eating behavior, and physical activity. Diabetes Care. 2001;24(1):117-123.

51. American College of Sports Medicine and the American Diabetes Association. Exercise and Type 2 Diabetes: American College of Sports Medicine and the American Diabetes Association: Joint Position Statement. Med Sci Sports Exerc. 2010;42(12):2282-2303.

52. American Diabetes Association. Diabetes mellitus and exercise. Diab Care 2002;25:s64-68.

53. Canadian Diabetes Association. Clinical practice guideline for the prevention and management of diabetes in Canada. Can J Diabetes. 2008;32(1):1-S201.

54. Moser A, van der Bruggen H, Widdershoven G. 2006. Competency in shaping one's life: autonomy of people with type 2 diabetes mellitus in a nurse-led, shared-care setting: A qualitative study. Intern J Nurs Stud. 2006;43(4):417-427.

55. Briel M, Young J, Tschudi P, Hugenschmidt C, Bucher HC, Langewitz W. Shared-decision making in general practice: do patients with respiratory tract infections actually want it? Swiss Med Wkly. 2007;137(33-34):483-485.

56. Degner LF, Kristjanson LJ, Bowman D, Sloan JA, Carriere KC, O'Neil J, Bilodeau B, Watson P, Mueller B. Information needs and decisional preferences in women with breast cancer. JAMA. 1997;277(18): 1485-1492.

57. Singh JA, Sloan JA, Atherton PJ, Smith T, Hack TF, Huschka MM, Rummans TA, Clark MM, Diekmann B, Degner LF. Preferred roles in treatment decision making among patients with cancer: a pooled analysis of studies using the Control Preferences Scale. AJMC. 2010;16(9):688-696.

58. Tariman JD, Berry DL, Cochrane B, Doorenbos A, Schepp K. 2010. Prefered and actual participation roles during health care decision making in persons with cancer: a systematic review. Ann Oncol. 2010;21:1145-1151.

59. Melnyk BM, Fineout-Overholt E. Evidence-Based Practice in Nursing \& Healthcare. Wolters Kluwer Lippincott Williams \& Wilkins, Philadephia. 2004.

60. Rycroft-Malone J, Seers K, Titchen A, Harvey G, Kitson A, McCormack B. What counts as evidence in evidence-based practice? J Adv Nurs. 2004;47(1);81-90.

61. Thompson C, McCaughan D, Cullum N, Sheldon TA, Mulhall A, Thompson DR. 2001. Research information in nurses' clinical decision-making: what is useful? J Adv Nurs. 2001;36(3):376-388.

62. Cote F, Gagnon J, Houme PK, Abdeljelil AB, Gagnon MP. 2012. Using the theory of planned behaviour to predict nurses' intention to integrate research evidence into clinical decision-making. J Adv Nurs. 2012;68(10):2289-2298.

63. Edwards A, Elwyn G. Shared Decision-Making in Health Care: Achieving Evidence-Based Patient Choice. University Press, Oxford. 2009. 
64. Zeitz K, McCutcheon H. Tradition, rituals and standards, in a realm of evidenced based nursing care. Contemp Nurse. 2005;18(3):300-308.

65. Larrabee JH, Janney MA, Ostrow CL, Withrow ML, Hobbs GRJ, Burant C. Predicting registered nurse job satisfaction and intent to leave. J Nurs Adm. 2003;33:271-283.

66. Melnyk BM, Fineout-Overholt E, Stillwell SB, Williamson KM. The seven steps of evidence-based practice: following this progressive, sequential approach will lead to improved health care and patient outcomes. Am J Nurs. 2010;110(1):51-53.

67. Thiel L, Ghosh Y. 2008. Determining registered nurses' readiness for evidence-based practice. Worldviews Evid Based Nurs. 2008:5(4):182-192.

68. Fineout-Overholt E, Melnyk BM, Schultz A. Transforming health care from the inside out: advancing evidence-based practice in the 21st century. J Prof Nurs. 2005;21(6):335-344.

69. Kajermo KN, Nordstrom G, Krusebrant A, Bjorvell H. 2000. Perceptions of research utilization: Comparisons between health care professionals, nursing students and a reference group of nurse clinicians. J Adv Nurs. 2000;31(1):99-109. 

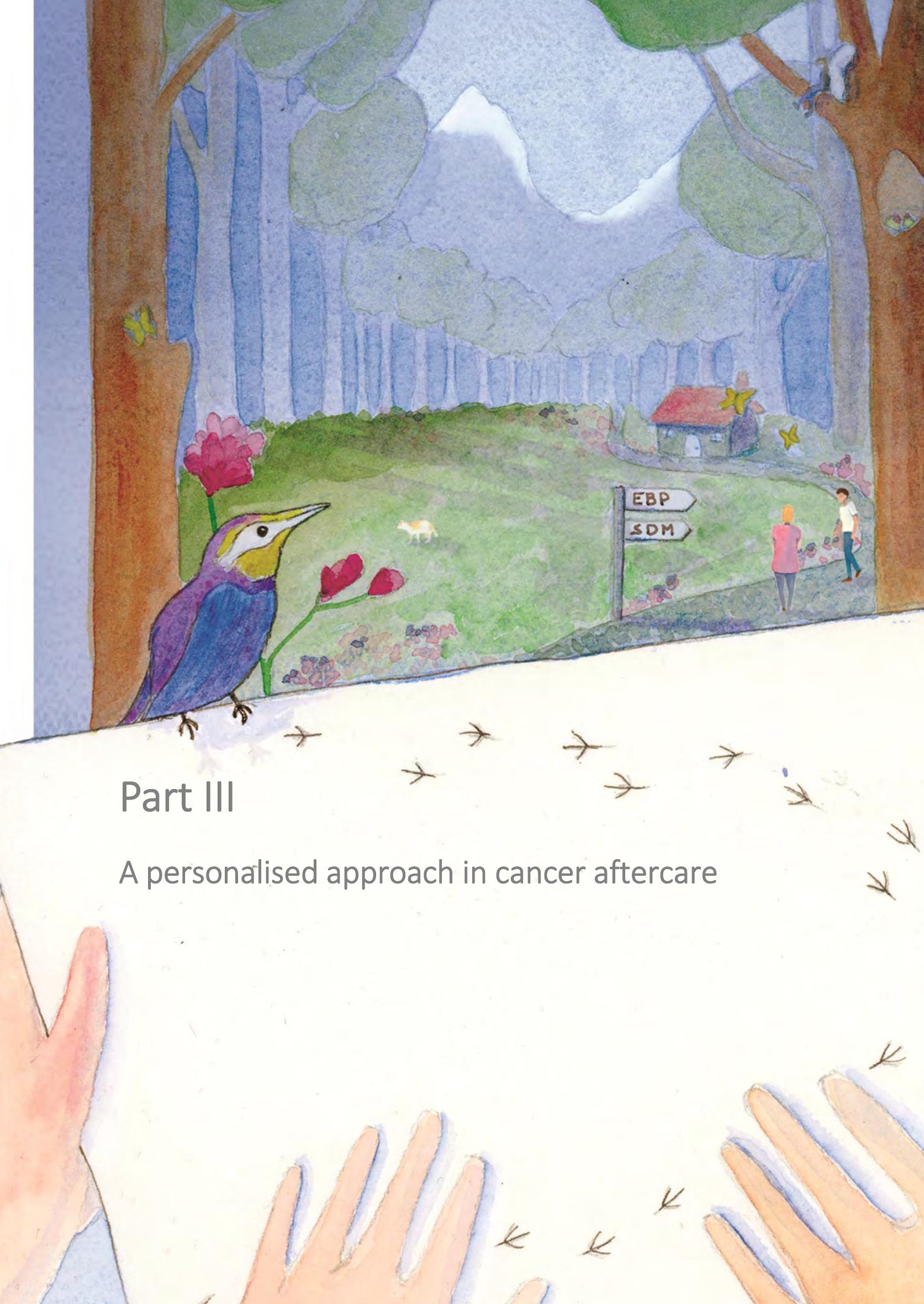
En dan ineens is er die klap,

die knal,

die ontreddering.

Je wilt ontwaken

uit die boze droom.

Voor slapen is geen tijd.

Knokken; voor je leven!

De controle leg je

in andere handen.

Mensen in witte pakken

kijken naar je lichaam.

Je voelt je naakt.

De verpakking lijkt

zoveel belangrijker,

dan jij dacht.

Gelaten tolereer je,

alles wat zij zeggen.

Het houvast voelt broos,

maar je hebt geen keus.

Je lijf holt achteruit.

Alsof je eerst zieker moet zijn,

voor herstel mogelijk is.

In de ogen van de mensen om je heen

probeer je te lezen

hoe het met je gaat.

Soms lukt dit.

Soms niet.

Slechts een enkeling

kijkt verder.

Niet alles wordt in getallen weergegeven.

Door alle waanzin heen

ben je ook nog mens.

$\mathrm{Al}$ is er weinig plek

voor gevoel.

Je moet dóór

als je wilt overleven.

Carine Pustjens 


\section{Chapter}

A conversation approach based on shared goal setting and shared decision making for nurses in cancer aftercare: a developmental study

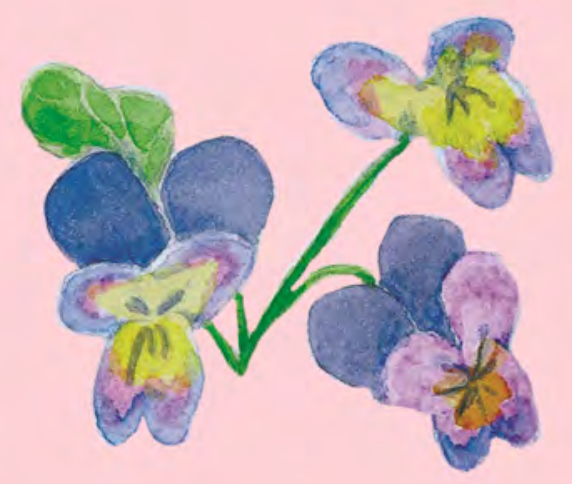

Jolanda H.H.M. Friesen-Storms Gerrie J.J.W. Bours Ingrid C.G. Snijders Trudy van der Weijden Kon-Siong G. (Asiong) Jie Anna J.H.M. Beurskens 


\section{Abstract}

\section{Purpose}

This study aimed to develop and pre-test a systematic conversation approach for nurses to tailor aftercare to oncology patient's goals, unmet needs and wishes.

\section{Methods}

We used an iterative developmental process for complex interventions: 1. Identifying problems 2 . Identifying overall objectives 3 . Designing the intervention 4. Pre-testing and adapting the intervention.

\section{Results}

The main results of the problem identification were: non-systematic and incomplete screening of potential issues, caveats in providing information, and shared decision-making. The overall objective formulated was: To develop a model for aftercare conversations based on shared goalsetting and decision-making.

The conversation approach consists of four phases: 1 . Preparation of the consultation including a questionnaire, 2. Shared goal-setting by means of a tool visualizing domains of life, and 3. Shared care planning by means of an overview of possible choices in aftercare, a database with health care professionals and a cancer survivorship care plan. 4. Evaluation.

The results of the pre-test revealed that the conversation approach needs to be flexible and tailored to the patient and practice setting, and embedded in the care processes. The conversation approach was perceived as enhancing patient-centeredness and leading to more in-depth consultations.

\section{Conclusion}

The conversation approach was developed in co-creation with stakeholders. The results of the pretest revealed important implications and suggestions for implementation in routine care.

Implications for cancer care: The aftercare conversation approach can be used by nurses to provide tailored patient-centered evidence-based aftercare. Tailored aftercare should support oncology patient's goals, unmet needs and wishes. Further tailoring is needed. 


\section{Introduction}

Cancer survival rates have improved due to advances in early detection and treatment. ${ }^{1,2}$ In conjunction with rising global cancer incidence rates, ${ }^{3}$ this has led to a rapid increase in the number of individuals living with and beyond cancer. In 2012, the estimated global 5-year prevalence for all cancers combined was 15,296 per 100,000 persons, equivalent to 32.6 million people living with a cancer history (within 5 years of diagnosis). ${ }^{4,5}$

Cancer and its treatment are often associated with long-term physical and psychosocial issues such as fatigue, pain, reduced muscle mass and strength, problems in daily functioning, fear, anxiety, sleep disturbances, reduced self-esteem and problems with returning to work. ${ }^{2,6-8}$, As such, cancer survivors often face the imposing task of rebuilding their physical and psychological health, which requires evidence-based aftercare. ${ }^{9,10}$ Oncology nurses and advanced practice nurses may play a crucial role in the development and delivery of coordinated aftercare. ${ }^{11}$

Aftercare is often intertwined with clinical follow-up, as these terms are not always clearly distinguished. In this study, aftercare and follow-up are defined in accordance with the Dutch Health Council ${ }^{9}$ as follows: aftercare includes interventions such as education, physical training and psychosocial support that helps patients to deal with potential issues of cancer and its treatment. Follow-up is the programmatic approach to prevent cancer recurrence.

According to Dutch's evidence-based clinical practice guidelines, aftercare should address the personal goals of the patient and stimulate his or her self-management. ${ }^{7,12}$

Guidelines recommend a systematic approach for aftercare based on shared decisionmaking, in which problems, goals, and wishes are systematically screened, shared, and integrated in a cancer survivorship plan ${ }^{7,13}$ Shared decision-making (SDM) actively involves patients in making health care choices based on the best available evidence, clinical expertise, and the values or goals of the informed patient. ${ }^{14-17}$ In chronic care, it is always important to include patients in making shared decisions about their health care management, which may, in turn, have positive effects on health and selfmanagement. $^{15,18}$

Very few cancer survivors receive any comprehensive aftercare, although a number of potential issues and needs have been identified for which evidence-based guidelines exist. These evidence-based guidelines do not seem to be consistently implemented ${ }^{11}$ and a majority of survivors report having unmet information and support needs. ${ }^{19-22}$ Patient advocacy groups, expert consensus panels, and governmental reports have recommended improvements in the quality of aftercare. ${ }^{2,9,10}$

Registered oncology nurses and advanced practice nurses can significantly contribute to the quality of aftercare. ${ }^{23}$ Nurses focus on teaching and control of potential issues, 
whereas physicians concentrate on medical diagnostics and therapy. Nurses also focus on personal goals and needs and are often more available than physicians to patients and families who call with problems associated with symptom management or home care support. $^{24}$

Although the relevance of goal-setting and shared decision-making in aftercare is acknowledged, nurses do not always know how to implement them in daily practice. ${ }^{15,25-27}$ In the literature, various models of how shared decision-making can be applied in daily practice have been developed. ${ }^{28-30}$ In addition, several authors describe models or guides for goal-setting. . $5,31,32$ These models, however, have not been specifically adjusted for cancer aftercare. It is important to develop a specific model or systematic conversation approach to facilitate shared goal-setting and shared decisionmaking in daily practice for cancer aftercare. ${ }^{7,9}$

The aim of this study was to develop and pre-test a systematic conversation approach for nurses to tailor aftercare to the oncology patients' goals, unmet needs, and wishes.

\section{Methods}

We applied an iterative development process for complex interventions based on a model proposed in the literature to develop a systematic conversation approach. ${ }^{33}$ The rationale for using this model was, firstly, a conversation approach for aftercare is a complex intervention that contains several interacting components. ${ }^{34}$ Interventions are complex if they request changes in individual health care providers' treatment approaches, in operational systems, and in the organization of care. ${ }^{35}$ Secondly, this model is based on a systematic review identifying the most comprehensive approach to the development of complex interventions for nursing practice. The development process consisted of the following steps: 1 . Identifying problems. 2. Identifying overall objectives of the intervention. 3. Designing the intervention based on theory and expert review. 4. Pretesting and adapting the intervention. Each step delivered input for the next step.

The development team consisted of four researchers and one oncologist. Two researchers are nurses (one $\mathrm{PhD}$ and one $\mathrm{PhD}$ student) and lecturers with expertise in EBP and SDM. One researcher is a medical doctor (PhD) and a professor in implementation of guidelines and an expert in SDM. One researcher is a physical therapist (PhD) and a professor in autonomy and participation for person's with a chronic illness and goal oriented measurement in patient care. The oncologist is also an associate professor in integrative patient centered health care. 
The setting and the specific methods of the development process are described in Figure 5.1 .

\section{Setting}

The setting of the study is a large urban 600 beds, teaching hospital in the South of the Netherlands. In the hospital there are several oncology care pathways, in which oncology nurses and nurse practitioners, provide a major contribution to the continuity of care during diagnosis, treatment, and aftercare. These care pathways include breast cancer, colorectal cancer, lung cancer, and malignant lymphoma.

\section{Step 1: Identifying problems}

In addition to the identification of aftercare problems in the literature, we identified problems in daily practice to tailor the development process to the setting.

First, 16 health care professionals that are experts in oncology care were invited to a brainstorming meeting in February 2013. In subgroups, they brainstormed about current and desired aftercare situations. The outcomes were discussed in a plenary session and summarized by the researcher (JF) after the meeting.

Second, individual in-depth interviews were conducted with patients $(n=8)$ and health care professionals $(n=9)$ about aftercare. A topic list was used based on the literature and on the results of the brainstorm meeting. Health care professionals and patients from different pathways were selected by purposive sampling in October 2013 and May 2014, respectively. Patients with different types of cancer (breast, malignant lymphoma and colorectal), variations in educational level, age, treatment, and duration after medical cancer treatment (from 6 weeks to 1 year) were selected by their health care professionals. The interviews were audiotaped and transcribed. The transcripts were analyzed in QSR NVIVO 10 software by directed content analysis. ${ }^{36}$ Two interviews were analyzed independently by two researchers. In cases of disagreement, the topics were discussed until consensus was reached. The other interviews were analyzed based on the topics agreed upon by the researchers. 


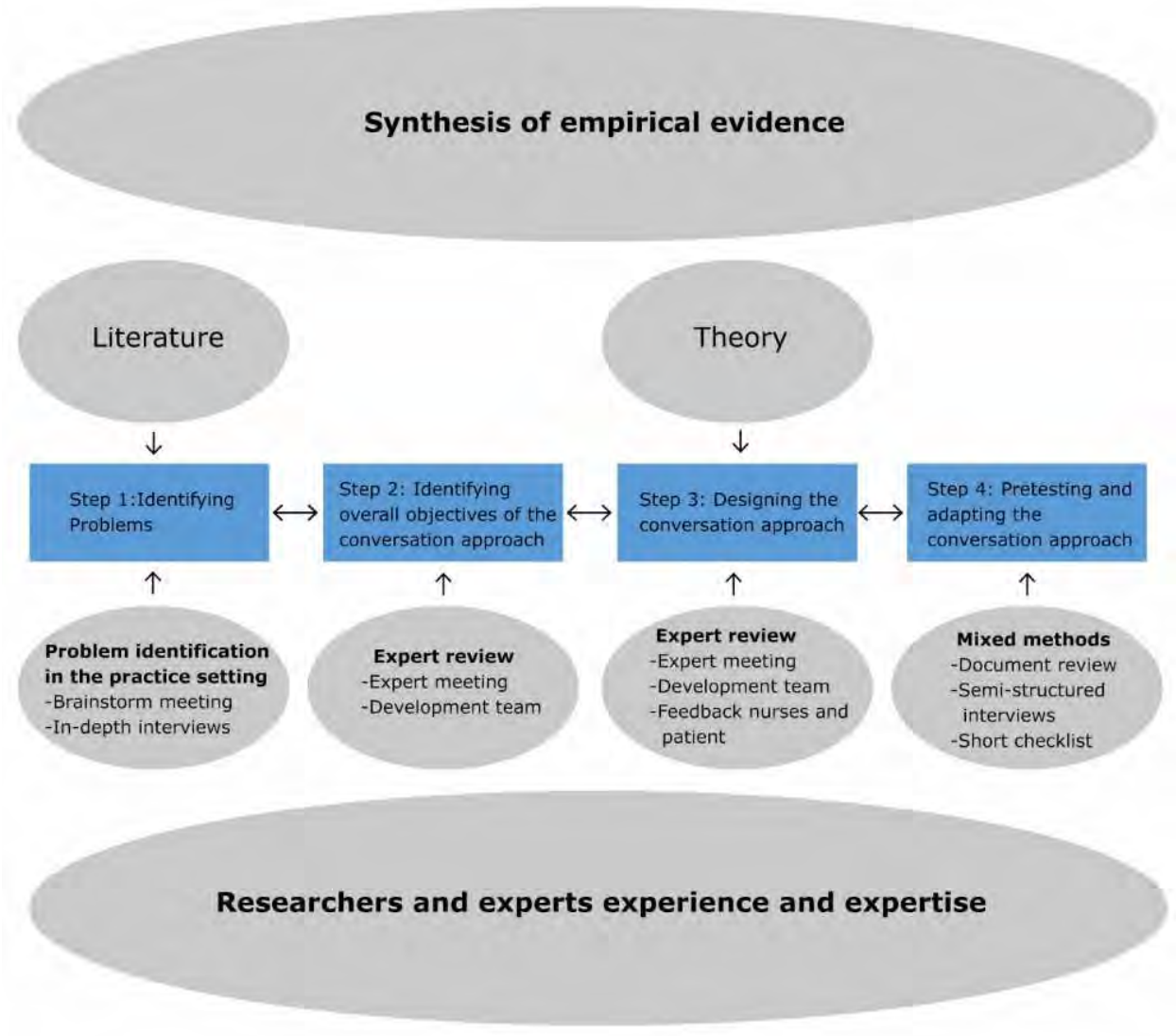

Figure 5.1 Development process for a conversation approach in cancer aftercare in nursing. ${ }^{33}$

\section{Step 2: Identifying overall objectives of the conversation approach}

The development team discussed, summarized, and prioritized the results from the problem identification step. These results were presented in a two-hour expert meeting in September 2014. Twenty-one health care professionals, mainly including the same individuals as the brainstorming meeting in the problem identification phase, were invited. In addition, two patients interviewed during the problem identification phase who were found to have a critical helicopter view, were invited. The findings of the problem identification phase were discussed within subgroups and finally, the development team summarized the results from the subgroups and formulated the objectives for improvement of the conversation approach. 


\section{Step 3: Designing the conversation approach based on expert review and theory}

After the expert meeting, the development team designed a first-draft of the conversation approach for an aftercare consultation based on evidence-based practice guidelines and the literature. The prototype conversation approach was discussed in winter 2014 with five health care professionals and with one patient from the expert meeting. The conversation approach was adapted to meet their wishes and needs.

\section{Step 4: Pre-testing and adapting the conversation approach}

The feasibility of the conversation approach was evaluated with patients $(n=11)$, three oncology nurses, and one nurse practitioner within two care pathways: breast cancer and malignant lymphoma. Feasibility was evaluated based on two areas of focus: acceptability and implementation of the intervention. ${ }^{37}$ Acceptability was determined by the satisfaction with the intervention. Implementation was measured by the levels of adherence to the principles of the approach and the reported barriers and facilitators. ${ }^{37}$ The nurses and the nurse practitioner first received instruction in the use of the conversation approach and practiced the approach in pairs. We did not expect nurses to use the phases and tools as a "cookbook", but rather as a flexible approach to support their consultations.

From April to June 2015, the nurses and nurse practitioner tested the conversation approach for aftercare consultation based on the principles of SDM during regular scheduled aftercare consultations. We used mixed methods.

To measure the levels of adherence we collected data by:

- Document review. We reviewed whether the patient and the nurse filled out the forms that were part of the conversation approach. In addition, the frequency of completed forms was counted.

- A short checklist. After each consultation, the nurse filled out a short checklist based on the conversation approach. The nurses could use three answer categories: yes, no, or partially.

To measure satisfaction and barriers and facilitators, we collected data by:

- A short checklist. On the same short checklist as for the level of adherence, the nurse assigned a score between 0-10 (0 being very unsatisfied and 10 being excellent satisfaction) for the aftercare consultation and registered how much time was needed to complete the consultation. 
Semi-structured interviews were conducted with all patients that gave informed consent $(n=9)$ and the oncology nurses and nurse practitioner. The interviews were conducted with a topic list based on the conservation approach and the literature. ${ }^{37-40}$ Additionally, the review of the forms from each individual patient was used for the interviews with the patients and a review of all patient forms for the interviews with the nurses. The interviews were audiotaped and transcribed by directed content analysis. ${ }^{36}$

\section{Ethical approval}

All procedures performed in studies involving human participants were in accordance with the ethical standards of the institutional and national research committee and with the 1964 Helsinki declaration and its later amendments or comparable ethical standards. Ethical approval for this study was obtained from the Medical Ethics Committee Zuyderland-Zuyd (no. 13-N-150 and 15-N-70).

Informed consent was obtained from all individual participants included in this study.

\section{Results}

\section{Step 1: Identifying problems}

Eleven cancer care professionals and three researchers attended the brainstorming meeting. Individually, we interviewed nine health care professionals and eight patients after cancer treatment. We interviewed four patients with malignant lymphoma, three with breast cancer and one with colorectal cancer. Patients were aged between 18 and 79 years with an average of 56 years.

The main results arising from both the brainstorm and interviews (Figure 5.2) were:

\section{Systematic screening of potential issues}

Patients experienced many issues after cancer treatment. The main issue was fatigue. Patients were screened with the Screening Inventory Psychosocial Problems questionnaire. ${ }^{41}$ However, screening was not conducted structurally in aftercare in all cancer pathways.

\section{Systematic provision of information}

Patients reported not always being informed about aftercare by health care professionals. 
Most health care professionals stated that it would be good to have aftercare conversations with patients in all care pathways. According to some health care professionals, the timing is important. These conversations should not be planned too early, as patients receiving information about aftercare during the diagnostic and intake phase, may suffer from information overload. However, issues related to cancer and its treatment should receive attention throughout the cancer pathway.

Some health care professionals mentioned that they knew which health care professionals were available within the hospital, but they did not have an overview of the available cancer care professionals in the region.

Patient (male, age 54, diagnosed with malignant lymphoma): "I got the idea for rehabilitation from people around me, not so much from Oncology. They asked if I was up for rehabilitation. What rehabilitation? I discussed it with Doctor (name) during the September check up. "Oh, you want to join that, that's fine". It was not offered actively, it was not mentioned, so I said I would want to do that."

At the intakes in October they found out that, in my case, physical condition was not really an issue. Which in itself is a major part of the rehabilitation program. My issue would be dealing with anxiety and depression."

Nurse 1: "Yes that is what we do. But, no more, and why? Knowingly we don't. Patients are already overburdened. When I look at the two patients this morning. One at $9 o^{\prime}$ clock, the other at 9:15. Who do not even manage to report at the proper ward. It's obvious not to overload them with information. It is no use going on about aftercare."

\section{Shared goal-setting and decision-making}

Some health care professionals and patients mentioned the importance of tailoring aftercare to the patient's needs and wishes. The decision-making process was diverse and dependent upon the health care professional.

The brainstorming meeting revealed that the values and wishes of the patient should be integrated in a survivorship care plan that is developed together with each patient. Health care professionals mentioned the following as important for the care plan: medical history, diagnosis, treatment, screening outcome, and a holistic summary of the aspects of life, patient goals, aftercare interventions, and referral and evaluation dates.

Patient (female, age 58, diagnosed with breast cancer): Yes, right, and everyone around me keeps telling me I should go for it (concerning rehabilitation), but I really hate fitness and swimming also. We also have a camper and regularly we take it out, we are thinking 
about going soon, we are going this weekend for a few days. If you sign up for rehabilitation, which you then have to do for three months, you are always dependent on the rehabilitation schedule"

Nurse 2: "I mean as health care professionals we sometimes want to shout it from the rooftops. Rehabilitation this that and the other and that is fine, it surely is, but a very large group are really not up for it".

\section{Barriers and facilitators of aftercare}

According to health care professionals and patients, the main barriers were: financial, if a treatment is not covered by health insurance it can be a problem for patients to undergo treatment, and the high workload and shortage of case managers. The management of aftercare could be improved: it would probably help to have oncology nurses as case managers on all cancer care pathways and to engage these nurses in aftercare. Health care professionals also mentioned that it would help to set up a network of primary and in hospital health care professionals within the region. Furthermore, there is a development that the responsibility for organizing aftercare is going to be transferred to the general practitioners. This, however, has not yet been organized and some major barriers, such as the possible lack of relevant oncological expertise among general practitioners, have not yet been resolved.

\section{Step 2: Identifying overall objectives of the conversation approach}

The expert meeting was attended by 11 cancer care professionals, two patients and three members of the development team. The two patients were treated for malignant lymphoma and had high levels of education.

Based on the data from step 1 (literature and practice analyses) the development team suggested developing an aftercare conversation approach based on the principles of SDM and presented the initial ideas. In three subgroups, the participants thought about the design of the conversation approach and the implementation in cancer care pathways. Based on the results of the expert meeting the development team formulated the following main objective (Figure 5.2): To develop a model for aftercare conversations based on shared goal-setting and shared decision-making during the first year of treatment (suggestion: 3, 6 months and 1 year after treatment). The main objective was further divided in six sub-objectives: 1 . To have patients prepare the consultation; 2 . To screen and prioritize potential issues related to cancer and its treatment; 3. To use visual tools to help the patient understand relevant information; 4. To give systematic information about aftercare; 5. To make a personalized survivorship care plan, created 
on evidence-based clinical practice guidelines; 6 . To provide health care professionals in the hospital with a social map of oncology care professionals in the region.

\section{Step 3: Designing the conversation approach based on expert review and theory}

The conversation approach consists of four phases as depicted in Figure 5.2.

During the development we cooperated with researchers who developed a conversation approach for shared goal-setting and decision-making for chronic patients in primary care. $^{42,43}$ We incorporated visual practical tools within the different phases of the approach that were mainly derived from existing tools (Figures 5.2 and 5.3).

The first three phases and tools are further explained in the text below. The fourth phase, Evaluation, is a basic phase in conversations.

\section{Preparation of the aftercare consultation}

The questionnaire to prepare the consultation was based on two currently used screening tools. The Dutch version of the Problem List (PL) (Cronbach's alpha total: 0.9) and Distress Thermometer (DT) (Correlation between DT and total score on PL: 0.68$)^{44,45}$ and the Screening Inventory Psychosocial Problems (Cronbach's alpha total: 0,91). ${ }^{41}$ In Dutch clinical practice guidelines, the Distress Thermometer is recommended as an overall screening instrument in aftercare. ${ }^{7,13}$ The Screening Inventory for Psychosocial Problems was developed and validated in the Dutch cancer setting ${ }^{41,46}$ and is the standard screening tool in our hospital. We added open questions to enable the patient to give his or her own opinion. We subdivided the questions into four main domains of life, derived from the International Classification of Functioning Disability and Health (World Health Organization) (functions, activities, participation, personal and external factors) to focus on all aspects of a person's life and to acknowledge the interrelation between health and health-related domains of life. The domains are 1. 'My Health', representing the patient's experiences with his health and health care problems, subdivided into physical, emotional and cognitive problems. 2 'My activities' representing the patient's experiences with daily activities and related problems. 3 'My environment' representing the patient's experiences with social relations, the physical environment and possible problems. 4 'My own way' representing the patient's current coping strategies and associated problems in coping. The domains of life also incorporate the three dimensions of 'work' involved in living with a chronic condition ${ }^{47}$ : My Health incorporates "illness work", My own Way incorporates "biographical work" and My Activities and My environment incorporate "everyday life work". 

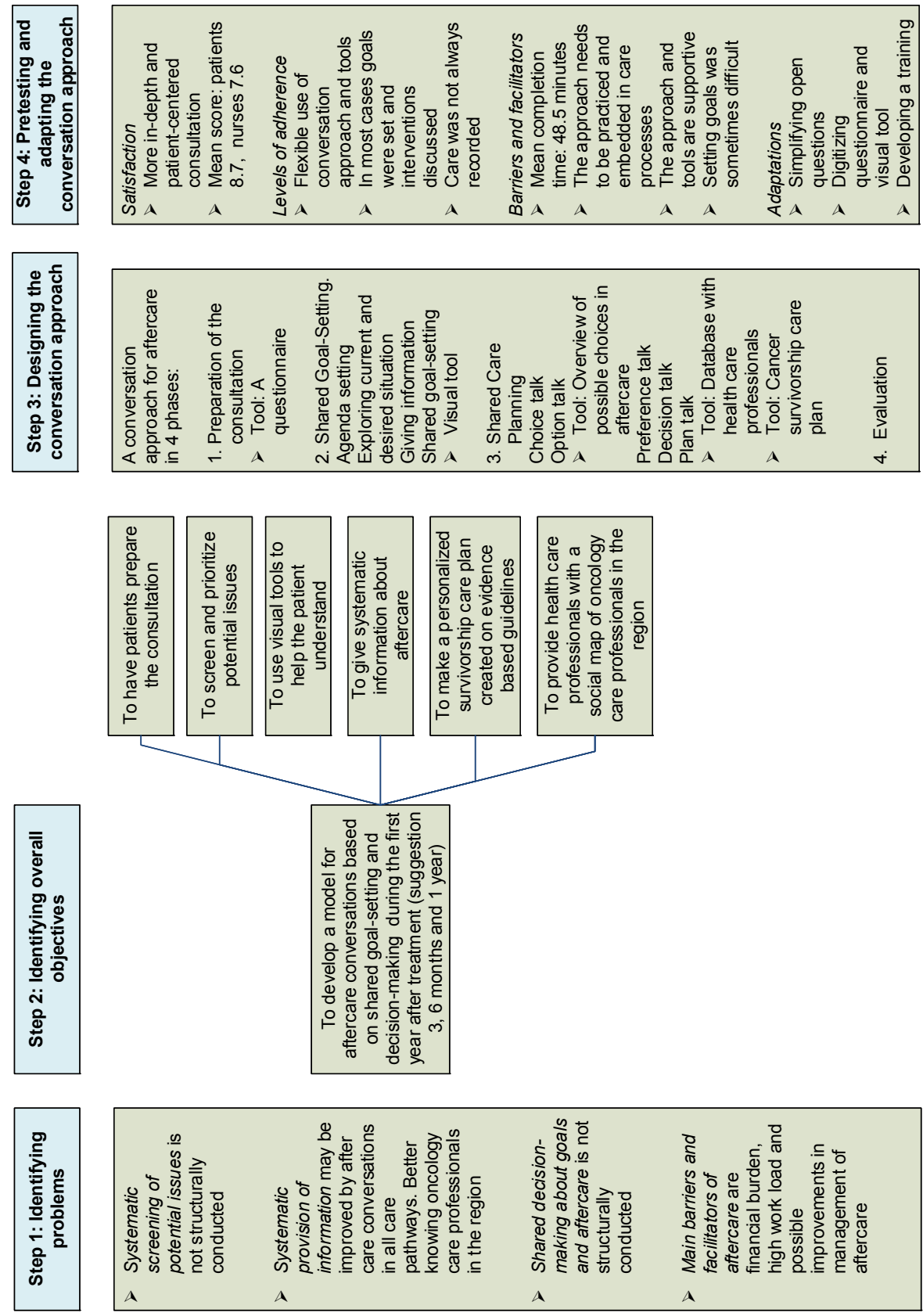

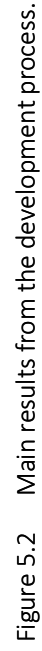




\section{Shared Goal-Setting}

After setting the agenda for the consultation, the results of the questionnaire are used in the goal-setting phase (Figure 5.2) to explore the current and desired situation together with the patient. If the current and desired situation deviate, goals are set with the patient. If not, no goals are set. To facilitate the dialogue between patient and health care professionals regarding problems and goals, a visual tool ${ }^{42}$ based on the same four domains of life, was incorporated (Figure 5.3).

\section{Shared Care Planning}

The Shared Care Planning phase was divided into a choice talk, an option talk, a preference talk, a decision talk and a plan talk ${ }^{28,43,48}$ (Figure 5.2). We used a shared decision-making model from Elwyn et al. ${ }^{28,48}$ as the basis for this phase. Although various SDM models have been developed, ${ }^{29}$ we chose this model because of its relative simplicity, which may make it applicable in clinical practice. The essential elements of the model include choice talk, option talk, preference talk and decision talk. In this model, choice talk involved clarifying that reasonable options are available to patients. Option talk refers to providing detailed information about the pros and cons of each option, preferences talk refers to eliciting patient's preferences and decision talk refers to supporting the process of considering the patient's preferences (including the patient's right to opt out of making a decision) and deciding on the best option; it is the deliberation.

In the choice talk of our model, the patient is informed about the choices that can be made to reach goals and the nurse explores the patient's knowledge regarding choices. In the option talk, the nurse discusses the different options with the patient including attributes, like impact on life style and resources, of the intervention options ${ }^{15}$ (for example exercising or physiotherapy to cope with fatigue). To support the option talk, an overview of possible aftercare choices was made based on an existing Stepped Care Model from the Netherlands Comprehensive Cancer Organization ${ }^{49}$ (Figure 5.3).

In the preference talk, the nurse supports the patient in phrasing wishes and preferences. In the decision talk the nurse and patient decide about aftercare interventions together. We added a plan talk to the model, in which the decisions are noted and concrete appointments are made. To support the plan talk, we incorporated a database developed by the Netherlands Comprehensive Cancer Organization (IKNL), with an overview of available cancer care professionals ("http://www.verwijsgidskanker.nl/") (Figure 5.3). In addition we developed the format of a care plan based on an aftercare plan example in an evidence-based guideline ${ }^{7}$ and on the opinion of health care professionals. 

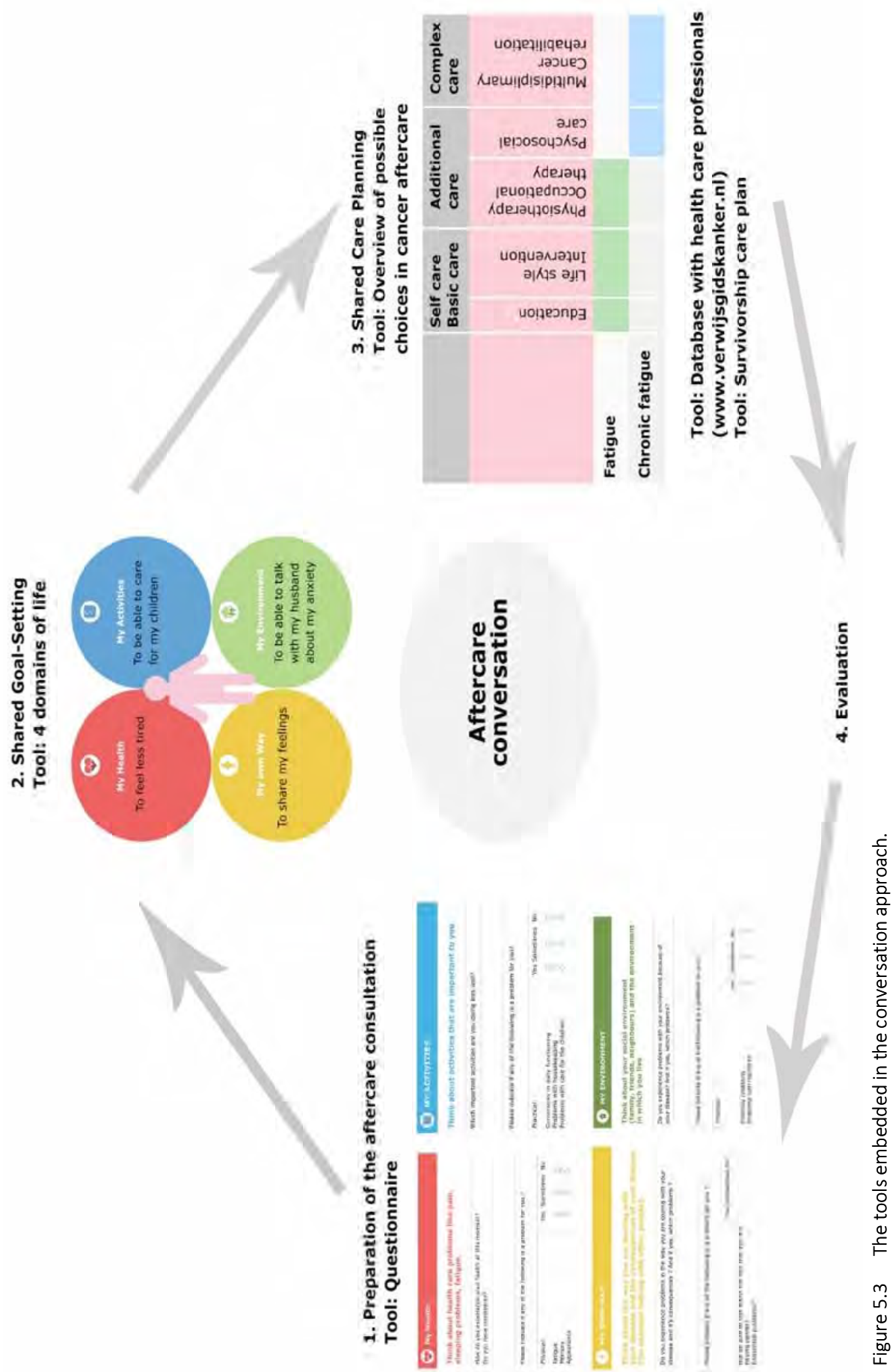


\section{Step 4: Pretesting and adapting the conversation approach}

The three oncology nurses and the nurse practitioner pretested the conversation approach with 11 patients. Nine patients were interviewed. We interviewed four patients with malignant lymphoma and five with breast cancer. The interviewed patients were between 44 and 88 years old, with an average age of 63 years. For one of these patients the short checklist was not completed by the nurse.

We structured the results of the pre-test according to the elements of feasibility, satisfaction, and implementation operationalized in levels of adherence and reported barriers and facilitators. To obtain insights into the barriers and facilitators of all phases of the conversation approach, we described general barriers and facilitators, and barriers and facilitators at each phase of the approach. We described adaptations made based on the results of satisfaction, and barriers and facilitators (see Figure 5.2).

\section{Satisfaction}

All nurses liked the aftercare consultations based on the conversation approach, because it supported them in a patient-centered approach and allowed more in-depth consultations.

The patients rated the consultation with a mean score of: 8.7 ( $\min 7.5$, max 10) with 0 for very unsatisfied and 10 for excellent satisfaction. The nurses rated the consultations with a mean score of: 7.6 ( $\min 7.0$ and $\max 8.0$ ).

Nurse 3: "What I like about this conversation model is that it creates an in-depth conversation. It doesn't become one of those conversations in which everything is just hunky dory."

Nurse 4: "Especially if you do that once a treatment has been completed. When you get them to figure out what to aim for, to set goals and a way of getting there and how we can support them. That, to me, appears to be the pinnacle of such a conversation."

Nurse 4: "What we mostly say is something along the lines of, what's important is how you feel now and what would you want to improve yourself, what's bothering you. Ahm. that's also the first time a patient is really asked how they are doing. The central topic is always the treatment and we are the ones suggesting things and then all of a sudden it turns around. You get a completely different atmosphere." 
Patient (female, age 55, diagnosed with breast cancer): "And then Uh.. right, then the conversation turns to, uhm, towards uhm, what's supposed to happen now and this and that and uhm.. It goes more like, uhm, what can we do for you and what's next."

\section{Levels of adherence}

See Table 5.1 for the results of the document review and the short checklist.

The conversation approach and tools were used in a flexible manner. In most cases goals were set, intervention options were discussed, and care was planned; however, the format of the patient care plan was not filled out for all patients.

Table 5.1 Document review and short checklist results.

\begin{tabular}{|c|c|c|}
\hline Phases of the conversation approach & $\begin{array}{l}\text { Document review } \\
\text { Frequency of yes } \mathrm{N} \\
\text { (total } n=11 \text { ) }\end{array}$ & $\begin{array}{c}\text { Checklist by nurses } \\
\text { Frequency of yes N } \\
\text { (total } n=10^{*} \text { ) }\end{array}$ \\
\hline \multicolumn{3}{|l|}{ 1. Preparation of aftercare consultation } \\
\hline Patient filled out questionnaire & 11 & 10 \\
\hline \multicolumn{3}{|l|}{ 2. Shared goal-setting } \\
\hline Explored current and desired situation & & 10 \\
\hline Set goals with patient & & $7^{\#}$ \\
\hline Used visual tool: "four domains of life" & 4 & \\
\hline \multicolumn{3}{|l|}{ 3. Shared care planning } \\
\hline Discussed different intervention options & & 9 \\
\hline Used the tool with possible post-treatment choices & & 6 \\
\hline Planned care with patient & & 9 \\
\hline Recorded care within patient care plan & 7 & 8 \\
\hline
\end{tabular}

* One checklist missing " Two missing values.

\section{General barriers and facilitators}

The completion time for the questionnaire was on average 12 minutes (SD 2 minutes, minimum 10, maximum 15 minutes).

The mean time needed to complete the aftercare consultation based on the checklists was 48.5 minutes (SD 21 minutes, minimum 15, maximum 90 minutes), including the time needed to complete the questionnaire.

Two nurses stated that the conversation approach took more time then they regularly have planned for an aftercare consultation. One nurse, however, said that if patients fill out the questionnaire at home and the nurses have more routine in the conversation approach, she thinks they might not need more than 30 minutes. Another nurse said she thinks they should structurally plan 45 minutes for an aftercare consultation to allow for 
a more in-depth consultation. All nurses said that the conversation approach needs to be practiced.

The timing of the aftercare consultation after the treatment differs between the malignant lymphoma and breast carcinoma pathways. In the breast carcinoma care pathway, aftercare consultations were regularly scheduled approximately three Months after treatment. The lymphoma care pathway recently reorganized aftercare and did not have a regularly scheduled time for aftercare consultations. During the pre-test in the lymphoma care pathway, all aftercare consultations bar one were conducted approximately three-weeks after treatment. One aftercare consultation was conducted three months after treatment, because the consultation had to be rescheduled several times. The "optimal" timing after treatment is not obvious.

To further implement the conversation approach, it needs to be embedded in care processes.

\section{Barriers and facilitators of different phases}

1. Preparation of the aftercare consultation

All patients and nurses perceived the questionnaire as strengthening the consultation. None of the patients interviewed found the questionnaire too extensive. Most patients thought it was easy to read with clear questions. Some patients needed help from a nurse or spouse because of age or cognitive problems. Some patients felt that not all questions were relevant to their personal situation, but they realized that this was inherent to questionnaires.

The patients had various opinions on the additional value of the open questions. Some patients found it difficult to respond, which was also confirmed by one nurse. The nurses thought the open questions were of additional value.

Patient (male, age 70, diagnosed with malignant lymphoma): "Well sometimes, but I don't mind, I can imagine uhm... such forms are drawn uhm.. not drawn up exclusively for me but for loads of people.

And obviously there are certain questions that don't apply to me personally."

\section{Shared Goal-Setting}

One nurse said that patients need help formulating goals. Some patients find it difficult to reflect on their situation and are sometimes very talkative, which makes exploring the current and desired situation a time-consuming process. Another nurse found it difficult to set goals. One nurse found it difficult to time the interruption needed to support the patient in determining problems and goals. 
The nurses liked using the visual tool "four domains of life" to facilitate the conversation, especially because the questionnaire is divided into the same domains. Two nurses mentioned that using the tool was not yet routine practice. Two patients explicitly stated that they found the visual tool supportive.

Patient (female, age 44, diagnosed with breast cancer): "Yes that's exactly how it uhm... well, it sure illustrates it rather nicely, my attitude towards it. It's spot on. The four main issues obviously, uhm.. Right, I quite like the look or rather the graphical illustration of the way that, well, what I think about all of this."

\section{Shared Care Planning}

All nurses said that they did not explicitly follow all steps of shared care planning. The nurses said that although they did not follow all steps of shared care planning, the separate steps were helpful in making the shared care planning more explicit. One nurse said that she maybe did not use the in-between steps because of her own preferences for aftercare.

Two nurses said that they found the aftercare plan on paper redundant, because they also have to report care in the electronic patient file.

Nurse 5: "Yes, however that is mostly my personal preference, I'm mostly in favor of rehabilitation, when you see someone fits a in group... is a team player. So when I come across someone more autonomous, working fulltime, than I think they should perhaps consider exercising with someone else at a local physical therapist. Find out if there is a program going on.

So that's it, that's what I think, talking about physical rehabilitation specifically that is. Or for example when someone has a knee injury and they should be swimming, then I'm mostly looking at the patient, but most days it's between rehabilitation and the physical therapist."

\section{Adaptations}

The analysis of the interviews revealed several important adaptations. The conversation approach needs to be flexible and tailored to the patient and practice setting.

Suggested alternatives to fill out the questionnaire are to send a questionnaire home before the consultation or to digitize the questionnaire and send a link to the patient. In addition, information about the goal of the consultation may be added in the invitation to fill out the questionnaire. Open questions in the questionnaire are sometimes difficult to fill out and could be simplified. 
To facilitate the use of the conversation approach and the tools, it seems important to digitize not only the questionnaire, but also other forms such as integrating the aftercare plan within the electronic patient files.

Conducting training sessions on the conversation approach and underlying theory seems necessary before further implementation.

We implemented the suggested adaptations accordingly: 1 . We simplified the open questions by integrating two open questions and by relating one question more to the disease. 2. We digitized the questionnaire and the visual tool. We added information about the goal of the consultation and the background of the questionnaire. Patients can fill out the questionnaire at home via a link and get an overview of the results depicted in the "four domains of life". Patients can print the results and send them via e-mail to the nurse. 3. We developed training on the conversation approach consisting of two meetings, with a month in-between where nurses can practice the approach in daily care. In addition, we made a short movie with an example of how to conduct the consultation based on the conversation approach.

\section{Discussion}

A conversation approach was created for aftercare in cancer survivorship. The approach developed is based on principles of shared goal-setting and shared decision-making and consists of four phases with supporting tools: 1. Preparation of the aftercare consultation using a questionnaire. 2 . Shared goal-setting by means of a tool visualizing domains of life, 3 . Shared care planning with an overview of possible choices in aftercare treatment related to particular issues, a database with available health care professionals, and an aftercare plan. 4. Evaluation. The conversation approach was systematically developed, pretested and adapted.

The pretest revealed that the conversation approach needs to be flexible, tailored to the patient and practice setting, and embedded in care processes. The conversation approach was perceived as enhancing patient centeredness and leading to more indepth consultations. However, an important challenge for the nurses was to elicit patient preferences and set goals. Another challenge was the time-consuming nature of the approach. 


\section{Results in context}

The developed conversation approach contains the main recommendations of evidencebased clinical practice guidelines regarding aftercare. ${ }^{7,12,13,45}$ Personalized care planning based on goal-setting and shared decision-making are strong elements of our approach, which has been shown to have positive effects on certain indicators of health status and capability for self-management. ${ }^{18}$

We believe that our study, which is focused on care decisions, adds to the current shared decision-making models in cancer that usually concern medical cure decisions, for example the choice between breast-conserving surgery or mastectomy as breast cancer treatments when both options have competing benefits and harms. ${ }^{50}$ Although other studies ${ }^{18,31,42,51,52}$ also use shared goal-setting and SDM in chronic care, our conversation approach is, to the best of our knowledge, the first approach that integrates goal-setting and SDM in cancer survivor aftercare.

Another important element of our conversation approach is the strong integration of supporting tools derived from currently used and existing tools from evidence-based clinical practice guidelines and the Netherlands Comprehensive Cancer Organization (IKNL). The graphic tool "four domains of life" is based on the framework of the ICF. Other studies ${ }^{31 / 42,53}$ also used a graphic tool based on the framework of the ICF. The holistic conversation approach and the four domains of life emphasize the recently developed concept of "positive health", in which health is not merely the absence of disease or disorders, but is defined as the ability to adapt to and to self-manage disorders or disease. ${ }^{54}$ Our four domains of life are rooted in this concept. ${ }^{55}$

Although the nurses and patients were positive about the approach, the pre-test revealed that eliciting patient preferences, applying patient-centered goal-setting and SDM is challenging. The difficulty and complexity of setting goals has been confirmed in other studies. The complexity of goal-setting is also recognized in primary care, ${ }^{56}$, in stroke rehabilitation, ${ }^{57}$ and in the work of allied health professions in general. ${ }^{26}$ In addition, studies confirm the complexity of eliciting patient preferences ${ }^{58}$ and implementing SDM in daily practice. ${ }^{16,59,60}$

The pre-test also revealed that the approach needs to be embedded within existing care processes. In a review of personalized care planning in chronic care, the effects seemed to be larger when the intervention was appropriately integrated within routine care. ${ }^{18}$ Another challenge revealed in our pretest is the time-consuming nature of our approach. Time is an important barrier known from implementation research. ${ }^{61}$ In daily practice and in research, there is growing attention for solely web-based interventions in aftercare without face-to-face contact with a health care professional. ${ }^{62-65}$ These 
interventions seem less time-consuming. However, the actual reach of web-based interventions is undiversified and predominantly reaches participants who are female, highly educated, white, and living in high-income countries. ${ }^{64,66}$ Although solely webbased interventions might provide valuable support in cancer aftercare, they will probably not reach all cancer survivors. In addition, web-based interventions might lack individualized care ${ }^{64}$ and our pretest showed that without the interpersonal support of a nurse or other health care professional, goal-setting and choosing aftercare might be complex tasks for patients.

From our study, the "optimal" timing of the approach in the cancer trajectory does not seem to be obvious. During the problem identification, health care professionals stated that these conversations should not be planned too early, as patients receiving information about aftercare during the diagnostic and intake phase tend to forget this information. However, care for cancer survivors should take place along the entire cancer care continuum, from diagnosis throughout the life span. ${ }^{11,68}$ For example, attention to psychosocial problems is needed during the treatment phase.

\section{Strengths and limitations}

The strength of this study is the use of the iterative developmental process for complex interventions in nursing, which involves intended users in the process. We started by identifying problems in current practice. Subsequently, we decided on the objectives for improvement and the conversation approach with oncology experts and two patient experts. We tailored the development of the conversation approach to the setting.

During the various phases of the study, we used purposive sampling to select health care professionals and patients. We strived for multi-stakeholder involvement and for data saturation. We may not have succeeded in all phases. For example, at the beginning of the practice analyses, the nurses selected mostly highly educated patients; so we asked them to purposeful also include patients with less education levels. Highly educated patients might be more competent in obtaining access to aftercare than patients with low education levels. In addition, during the problem identification we may not have reached data saturation for all stakeholder groups. For example, we only interviewed eight patients from three different cancer care pathways.

During the development process, two patients were invited to the expert meeting. We purposefully chose patients we thought would have a helicopter view. Both of these patients were highly educated, male and treated for malignant lymphoma. We might have chosen more patients with different education levels, sex and diagnoses.

In order to incorporate sufficient perspectives, we applied triangulation by searching the literature ${ }^{19-22,68}$ and we used an iterative developmental process. 
To improve trustworthiness, we also carried out peer debriefing of the main results of the problem identification and pretest within the development team. We conducted a member check for the results from the problem identification at an expert meeting (consisting of some of the interviewed health care professionals and two interviewed patients) and during individual conversations with health care professionals that did not attend the expert meeting, wherein the results were confirmed. The results of the pretest were discussed with the nurse practitioner and nurses that participated in the pretest.

A limitation of the pretest may be that it was conducted within two cancer pathways that already had embedded aftercare consultations as part of their care chain and not in the other cancer pathways. This may have positively influenced the results of the pretest, because implementation within these care pathways may be more feasible than in other care pathways.

\section{Recommendations for practice and further research}

One of our future challenges is to provide nurses and nurse practitioners sufficient support to elicit patient preferences, apply shared goal-setting and decision-making, and to further tailor the approach to make it more flexible and facilitate integration within routine care. Giving patients the opportunity to fill out the questionnaire at home, either digitally or on paper, might support the implementation of the approach, as it is less time-consuming for the nurse. Providing nurses with training and on-the-job coaching might support the nurses in implementing the approach.

Within the care organization, having oncology nurses as case managers engaged in aftercare and having a network of health care professionals occupied with cancer care within the region, would facilitate further implementation. In addition, developing models in which hospitals and general practitioners share aftercare seems important for further development of aftercare. ${ }^{69,70}$ The developed aftercare conversation approach could also be tailored for use in primary care.

In the pre-test, we evaluated the feasibility of the conversation approach. Patients seemed satisfied with the approach. However, further research is needed to obtain deeper insight into what is actually on the agenda during the conversation approach and investigate patient's experiences of the approach. These insights might help to further tailor and embed the approach. Furthermore, after additional tailoring and embedding of the approach within care processes, conducting a process evaluation and an effectiveness study is the next step to study the implementation and its' effects. Outcomes may be determined by measuring the degree of SDM using SDMQ- $9^{71}$ and the CollaboRATE, ${ }^{72}$ for instance. 


\section{Conclusion}

In this study, we describe the development and pre-test of a conversation approach based on shared goal-setting and shared decision-making for nurses in cancer aftercare. Our model was co-created with important aftercare stakeholders, incorporates evidence-based cancer care guidelines and supports patient-centered care. The results of the pre-test revealed important implications and suggestions for implementation, such as tailoring and embedding the approach within care processes and providing nurses with training and on-the-job coaching. In future, further evaluation of the conversation approach in practice is needed.

\section{Implications for cancer care}

The aftercare conversation approach can be used by nurses and other health care professionals to provide tailored patient-centered evidence-based aftercare. Tailored aftercare should support oncology patient's goals, unmet needs and wishes. Further tailoring aftercare in future is needed.

\section{Acknowledgments}

We would like to thank all participating patients and health care professionals and especially the participating nurses from the breast cancer and malignant lymphoma care pathways of the Zuyderland Medical Center (Former Atrium Medical Center Heerlen) for their contributions to this study. Furthermore, we would like to thank senior-advisor Suzanne Verboort-Bogers (MMI) of the Netherlands Comprehensive Cancer Organization (IKNL) for her support with our study and Guido Scholl for his support with translating the quotes of the respondents. 


\section{References}

1. American Cancer Society, 2015. Global Cancer Facts \& Figures., 3rd ed. American Cancer Society, Atlanta. European Society for Medical Oncology (ESMO), 2014. Rehabilitation issues during cancer treatment and follow-up. ESMO Press, Viganello-Lugano.

2. European Society for Medical Oncology (ESMO), 2014. Rehabilitation issues during cancer treatment and follow-up. ESMO Press, Viganello-Lugano.

3. Torre LA, Siegel RL, Ward EM, Jemal A. Global Cancer Incidence and Mortality Rates and Trends-An Update. Cancer Epidemiol Biomarkers Prev. 2016;25:16-27.

4. Corsini N, Fish J, Ramsey I, Sharplin G, Flight I, Damarell R, Wiggins B, Wilson C, Roder D, Eckert M. Cancer survivorship monitoring systems for the collection of patient-reported outcomes: a systematic narrative review of international approaches. J Cancer Surviv. 2017;11:486-497.

5. Ferlay J, Soerjomataram I, Dikshit R, Eser S, Mathers C, Rebelo M, Parkin DM, Forman D, Bray F. Cancer incidence and mortality worldwide: sources, methods and major patterns in GLOBOCAN 2012. Int. J. Cancer. 2015;136:E359-386.

6. Aaronson NK, Mattioli V, Minton O, Weis J, Johansen C, Dalton SO, Verdonck-de Leeuw IM, Stein KD, Alfano CM, Mehnert A, de Boer A, van de Poll-Franse LV. 2014. Beyond treatment - Psychosocial and behavioural issues in cancer survivorship research and practice. EJC Suppl. 2014;12:54-64.

7. Integraal Kankercentrum Nederland [Comprehensive Cancer Centre the Netherlands ] (IKNL), 2011. Richtlijn Herstel na Kanker [Guideline Cancer survivorship care]. Retrieved from: http://www.oncoline.nl/herstel-na-kanker. (Accessed 5 January 2013).

8. Simard S, Thewes B, Humphris G, Dixon M, Hayden C, Mireskandari S, Ozakinci G. Fear of cancer recurrence in adult cancer survivors: a systematic review of quantitative studies. J. Cancer Surviv. 2013; 7:300-322.

9. The Health Council of the Netherlands, 2007 Follow-up in oncology. Identify objectives, substantiate actions. The Hague: Health Council of the Netherlands; 2007. Retrieved from: https://www.gezondheidsraad.nl/en/task-and-procedure/areas-of-activity/optimum-healthcare/followup-in-oncology-identify-objectives. (Accessed 5 January 2013).

10. Hewitt M, Greenfield S, Stovall EL. 2005. From Cancer Patient to Cancer Survivors: Lost in Transition. Institute of Medicine, Washington DC.

11. Klemp JR. Survivorship care planning: one size does not fit all. Semin Oncol Nurs. 2015;31:67-72.

12. Visserman EA, Gijsen BCM, Blaauwbroek HG. 2014. Zorgstandaard Kanker [Care Standard for Cancer]. Leven met Kanker [Living with cancer], IKNL, KWF Kankerbestrijding, Utrecht.

13. Integraal Kankercentrum Nederland [Comprehensive Cancer Centre the Netherlands] (IKNL), 2011 b.

Richtlijn Oncologische revalidatie [Guideline Oncological Rehabilitation]. Retrieved from:

http://www.oncoline.nl/oncologische-revalidatie. (Accessed 5 January 2013).

14. Charles C, Gafni A, Whelan T. Shared decision-making in the medical encounter: What does it mean? (or it takes at least two to tango). Soc Sci Med. 1997;44:681-692.

15. Friesen-Storms JH, Bours GJ, Weijden T vd, Beurskens AJ. 2015. Shared decision making in chronic care in the context of evidence based practice in nursing. Int J Nurs Stud. 2015;52:393-402.

16. Légaré F, Stacey D, Turcotte S, Cossi MJ, Kryworuchko J, Graham ID, Lyddiatt A, Politi MC, Thomson R, Elwyn G. Interventions for improving the adoption of shared decision making by healthcare professionals. Cochrane Database Syst Rev. 2014;9:1-45.

17. Weston WW. 2001. Informed and shared decision-making: the crux of patient-centered care. CMAJ 2001;165:438-439.

18. Coulter A, Entwistle VA, Eccles A, Ryan S, Shepperd S, Perera R. Personalised care planning for adults with chronic or long-term health conditions. Cochrane Database Syst Rev. 2015;3:1-131.

19. Kotronoulas G, Papadopoulou C, Burns-Cunningham K, Simpson M, Maguire R. A systematic review of the supportive care needs of people living with and beyond cancer of the colon and/or rectum. Europ J Oncol Nurs. 2017;29:60-70.

20. Paterson C, Robertson A, Smith A, Nabi G. Identifying the unmet supportive care needs of men living with and beyond prostate cancer: A systematic review. Eur J Oncol Nurs. 2015;19:405-418. 
21. Rowlands IJ, Janda M, McKinnon L, Webb PM, Beesley VL. 2015. Prevalence, predictors, and correlates of supportive care needs among women 3-5 years after a diagnosis of endometrial cancer. Support Care Cancer. 2015;23:1205-1214.

22. Willems RA, Bolman CA, Mesters I, Kanera IM, Beaulen AA, Lechner L. 2016. Cancer survivors in the first year after treatment: the prevalence and correlates of unmet needs in different domains. Psychooncology 2016;25:51-57.

23. Corcoran S, Dunne M, McCabe MS. The Role of Advanced Practice Nurses in Cancer Survivorship Care. Semin Oncol Nurs. 2015;31:338-347.

24. Schulman LN. Efficient and Effective Models for Integrating Advanced Practice Professionals into Oncology Practice, ASCO EDUCATIONAL BOOK: PROFESSIONAL DEVELOPMENT Optimizing the Role of Advanced Practice Professionals in Oncology Practice. ASCO. 2013; e377-e379.

25. Bekelman DB, Johnson-Koenke R, Ahluwalia SC, Walling AM, Peterson J, Sudore RL. Development and Feasibility of a Structured Goals of Care Communication Guide. J Palliat Med. 2017;20:1004-1012

26. Marsland E, Bowman J. An interactive education session and follow-up support as a strategy to improve clinicians' goal-writing skills: a randomized controlled trial. J Eval Clin Pract. 2010;16:3-13.

27. Revello K, Fields W. An Educational Intervention to Increase Nurse Adherence in Eliciting Patient Daily Goals. Rehabil Nurs. 2015;40(5):320-326.

28. Elwyn G, Frosch D, Thomson R, Joseph-Williams N, Lloyd A, Kinnersley P, Cording E, Tomson D, Dodd C, Rollnick S, Edwards A, Barry M. Shared Decision Making: A Model for Clinical Practice. J Gen Intern Med. 2012;27:1361-1367.

29. Makoul G, Clayman ML. An integrative model of shared decision making in medical encounters. Patient Educ Couns. 2006;60:301-312.

30. O'Connor A, Stacey D, Jacobsen MJ. 2007. Ottowa Decision Support Tutorial. Retrieved from: https://decisionaid.ohri.ca/ODST/pdfs/ODST.pdf. (Accessed 4 May 2014).

31. Lenzen SA, Daniels R, van Bokhoven MA, van der Weijden T, Beurskens A. Setting goals in chronic care: Shared decision making as self-management support by the family physician. Eur J Gen Pract. 2015;21: 138-144.

32. Scobbie L, Dixon D, Wyke S. Goal setting and action planning in the rehabilitation setting: development of a theoretically informed practice framework. Clin Rehabil. 2011;25:468-482.

33. Corry $\mathrm{M}$, Clarke $\mathrm{M}$, While $\mathrm{AE}$, Lalor J. Developing complex interventions for nursing: a critical review of key guidelines. J Clin Nurs. 2013;22:2366-2386.

34. Craig P, Dieppe P, Macintyre S, Michie S, Nazareth I, Petticrew M. Developing and evaluating complex interventions: the new Medical Research Council guidance. BMJ. 2008;337:a1655.

35. Lau R, Stevenson F, Ong BN, Dziedzic K, Treweek S, Eldridge S, Everitt H, Kennedy A, Qureshi N, Rogers A, Peacock R, Murray E. Achieving change in primary care--effectiveness of strategies for improving implementation of complex interventions: systematic review of reviews. BMJ Open. 2015;5:e009993.

36. Hsieh HF, Shannon SE. Three approaches to qualitative content analysis. Qual Health Res. 2005;15: 1277-1288.

37. Bowen DJ, Kreuter M, Spring B, Cofta-Woerpel L, Linnan L, Weiner D, Bakken S, Kaplan CP, Squiers L, Fabrizio C, Fernandez M. How We Design Feasibility Studies. Am J Prev Med. 2009;36:452-457.

38. Kothe EJ, Mullan BA. Acceptability of a theory of planned behaviour email-based nutrition intervention. Health Promot Int. 2014;29:81-90.

39. Stevens A, Beurskens A, Koke A, van der Weijden T. The use of patient-specific measurement instruments in the process of goal-setting: a systematic review of available instruments and their feasibility. Clin Rehabil. 2013;27:1005-1019.

40. Vandelanotte C, De Bourdeaudhuij I. Acceptability and feasibility of a computer-tailored physical activity intervention using stages of change: project FAITH. Health Educ Res. 2003;18:304-317.

41. Braeken AP, Lechner L, van Gils FC, Houben RM, Eekers D, Ambergen T, Kempen G. The effectiveness of the Screening Inventory of Psychosocial Problems (SIPP) in cancer patients treated with radiotherapy: design of a cluster randomised controlled trial. BMC Cancer. 2009;9:1471-2407. 
42. Lenzen SA. 2015. Het vier bollen model [The four circle model]. Retrieved from: https://www.zuyd.nl/ onderzoek/lectoraten/autonomie-en-

participatie/ /media/Files/Onderzoek/Kenniskring\%20Autonomie\%20en\%20participatie\%20van\%20chro nisch\%20zieken/Het\%204-\%20bollen\%20model\%20voor\%20website\%20121015.pdf（Accessed 12 October 2015).

43. National Health Care Institute, 2015. Gezamenlijke besluitvorming over doelen en zorgafspraken. [Shared Decision Making about Goals and Care Interventions]. Retrieved from:

https://www.zorginstituutnederland.nl/publicaties/publicatie/2015/10/15/gezamenlijke-besluitvormingover-doelen-en-zorgafspraken-handreiking (Accessed 15 October 2015).

44. Tuinman MA, Gazendam-Donofrio SM, Hoekstra-Weebers JE. Screening and referral for psychosocial distress in oncologic practice: use of the Distress Thermometer. Cancer. 2008;113:870-878.

45. Integraal Kankercentrum Nederland [Comprehensive Cancer Centre the Netherlands] (IKNL), 2017. Richtlijn: detecteren behoefte psychosociale zorg [Guideline: Detecting the need for psychosocial care]. Retrieved from: http://www.oncoline.nl/detecteren-behoefte-psychosociale-zorg. (Accessed 24 April 2018).

46. Braeken AP, Lechner L, Houben RM, Van Gils FC, Kempen GI. Psychometric properties of the Screening Inventory of Psychosocial Problems (SIPP) in Dutch cancer patients treated with radiotherapy. Eur J Cancer Care. 2011;20:305-314.

47. Corbin J, Strauss A. Managing Chronic Illness at Home: Three Lines of Work. Qualitative Sociology 1985;8:224-47.

48. Elwyn G, Lloyd A, May C, van der Weijden T, Stiggelbout A, Edwards A, Frosch DL, Rapley T, Barr P, Walsh T, Grande SW, Montori V, Epstein R. Collaborative deliberation: A model for patient care. Patient Educ Couns. 2014;97:158-164.

49. Integraal Kankercentrum Nederland [Comprehensive Cancer Centre the Netherlands ] (IKNL), 2015. Gepaste nazorg in de oncologie: werkafspraken in de keten: Nazorgprogramma voor patiënten met melanoom. [Aftercare in Oncology: Appropriate aftercare in oncology: working arrangements in the chain: Post-treatment program for patients with melanoma].

50. Stacey D, Legare F, Col NF, Bennett CL, Barry MJ, Eden KB, Holmes-Rovner M, Llewellyn-Thomas H, Lyddiatt A, Thomson R, Trevena L, Wu JH. Decision aids for people facing health treatment or screening decisions. . Cochrane Database Syst Rev. 2014;1-331.

51. van de Pol MH, Fluit CR, Lagro J, Slaats YH, Olde Rikkert MG, Lagro-Janssen AL. Expert and patient consensus on a dynamic model for shared decision-making in frail older patients. Patient Educ Couns. 2016;99:1069-1077.

52. van de Pol MH, Fluit CR, Lagro J, Slaats Y, Olde Rikkert MG, Lagro-Janssen AL. Shared decision making with frail older patients: Proposed teaching framework and practice recommendations. Gerontol Geriatr Educ. 2016;1-14.

53. Murphy J, Boa S. Using the WHO-ICF with talking mats to enable adults with long-term communication difficulties to participate in goal setting. Augment Altern Commun. 2012;28(1):52-60.

54. Huber M, Knottnerus JA, Green L, van der Horst H, Jadad AR, Kromhout D, Leonard B, Lorig K, Loureiro MI, van der Meer JW, Schnabel P, Smith R, van Weel C, Smid H. How should we define health? BMJ. 2011;343:d4163.

55. Institute for Positive Health. Het spinnenweb model [The Spider Web Model]. Retrieved from: http://www.ipositivehealth.com/. (Accessed 8 July 2017).

56. Lenzen SA, van Dongen JJ, Daniëls R, van Bokhoven MA, van der Weijden T, Beurskens A. What does it take to set goals for self-management in primary care? A qualitative study. Fam Pract. 2016;33:698-703.

57. Rosewilliam S, Roskell CA, Pandyan AD. A systematic review and synthesis of the quantitative and qualitative evidence behind patient-centred goal setting in stroke rehabilitation. Clin Rehabil. 2011;25: 501-514.

58. Klaassen L, Dirksen C, Boersma L, Hoving C. Developing an aftercare decision aid; assessing health professionals' and patients' preferences. Eur J Cancer Care. 2017;e12730.

59. Blair L, Legare F. Is Shared Decision Making a Utopian Dream or an Achievable Goal? Patient. 2015;8: 471-476. 
60. Elwyn G, Frosch DL, Kobrin S. Implementing shared decision-making: consider all the consequences. Implement Sci. 2016;11:114.

61. Grol R, Grimshaw J. From best evidence to best practice: effective implementation of change in patients' care. Lancet. 2003;362:1225-1230.

62. Duman-Lubberding S, van Uden-Kraan CF, Jansen F, Witte BI, van der Velden LA, Lacko M, Cuijpers P, Leemans CR, Verdonck-de Leeuw IM. Feasibility of an eHealth application "OncoKompas" to improve personalized survivorship cancer care. Support Care Cancer. 2016;24:2163-2171.

63. Kanera IM, Bolman CA, Willems RA, Mesters I, Lechner L. Lifestyle-related effects of the web-based Kanker Nazorg Wijzer (Cancer Aftercare Guide) intervention for cancer survivors: a randomized controlled trial. J Cancer Surviv. 2016;10:883-897.

64. Post KE, Flanagan J. Web based survivorship interventions for women with breast cancer: An integrative review. Europ J Oncol Nurs. 2016;25:90-99.

65. Willems RA, Bolman CA, Mesters I, Kanera IM, Beaulen AA, Lechner L. The Kanker Nazorg Wijzer (Cancer Aftercare Guide) protocol: the systematic development of a web-based computer tailored intervention providing psychosocial and lifestyle support for cancer survivors. BMC Cancer. 2015;15:580.

66. Kohl LF, Crutzen R, de Vries NK. Online prevention aimed at lifestyle behaviors: a systematic review of reviews. J Med Internet Res. 2013;15:e146.

67. National Coalition for Cancer Survivorship. Our mission. Retrieved from: http://www.canceradvocacy.org/about-us/our-mission/. (Accessed 11 September 2017).

68. Pratt-Chapman M, Simon MA, Patterson AK, Risendal BC, Patierno S. Survivorship navigation outcome measures: a report from the ACS patient navigation working group on survivorship navigation. Cancer. 2011;117:3575-3584.

69. Hall SJ, Samuel LM, Murchie P. Toward shared care for people with cancer: developing the model with patients and GPs. Fam Pract. 2011;28:554-564.

70. Jefford M, Rowland J, Grunfeld E, Richards M, Maher J, Glaser A. Implementing improved post-treatment care for cancer survivors in England, with reflections from Australia, Canada and the USA. Br. J. Cancer 2013;108:14-20.

71. Kriston L, Scholl I, Holzel L, Simon D, Loh A, Harter M. The 9-item Shared Decision Making Questionnaire (SDM-Q-9). Development and psychometric properties in a primary care sample. Patient Educ Couns. 2010;80:94-99.

72. Elwyn G, Barr PJ, Grande SW, Thompson R, Walsh T, Ozanne EM. Developing CollaboRATE: A fast and frugal patient-reported measure of shared decision making in clinical encounters. Patient Educ Couns. 2013;93:102-107. 



\section{Chapter}

Perceived facilitating and restricting factors for

Healthcare Professionals to Adopting a

\section{Patient Decision Aid for Breast Cancer}

Aftercare: a cross-sectional study

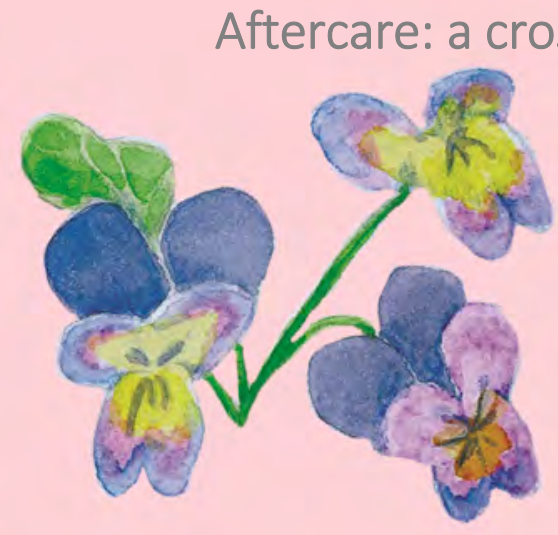

Linda A. Klaassen

Jolanda H.H.M. Friesen- Storms

Gerrie J.J.W. Bours

Carmen D. Dirksen

Liesbeth J. Boersma

Ciska Hoving

Under review: minor revisions. Patient Education and Counseling 


\section{Abstract}

\section{Objective}

Effective healthcare innovations are often not adopted and implemented. An implementation strategy based on facilitators and barriers for use as perceived by healthcare professionals could increase adoption rates. This study therefore aimed to identify the most relevant facilitators and barriers for use of an innovative breast cancer aftercare decision aid (PtDA) in healthcare practice.

\section{Methods}

Facilitators and barriers (related to the PtDA, adopter and healthcare organisation) were assessed among breast cancer aftercare health professionals $(n=81)$, using the MIDI questionnaire. For each category, a backward regression analysis was performed (dependent $=$ intention to adopt). All significant factors were then added to a final regression analysis to identify to most relevant determinants of PtDA adoption.

\section{Results}

Expecting higher compatibility with daily practice and clinical guidelines, more positive outcomes of use, higher relevance for the patient and increased application self-efficacy were significantly associated with a higher intention to adopt. Self-efficacy and patient relevance remained significant in the final model.

\section{Conclusions}

Low perceived application self-efficacy and patient relevance are the most important barriers for health professions to adopt a breast cancer aftercare PtDA.

\section{Practice implications}

To target application self-efficacy and PtDA patient relevance, the implementation strategy could apply health professional peer champions. 


\section{Introduction}

In the Netherlands the number of women eligible for aftercare after completing curative treatment for breast cancer has increased in the last decade. ${ }^{1}$ Within the national breast cancer guidelines it is recommended to personalize aftercare based on individual patients' problems, preferences and goals in an individual cancer survivorship plan. ${ }^{2-4}$ To ensure patient involvement in decision making processes such as these, Shared Decision Making (SDM) is often used. SDM is a form of patient- caregiver communication in which the patient and the caregiver reach decisions concerning healthcare or treatment on an equal basis. It has been shown that patient decision aids improve the process of SDM and reduce decisional conflict in treatment choices. ${ }^{5}$

To support SDM in breast cancer patients choosing their aftercare ${ }^{6-8}$, an aftercare Patient Decision Aid (PtDA) has been developed by our group. ${ }^{9}$ The PtDA was tested in a pilot study and the results showed a small improvement in perceived SDM, with more patients choosing less intensive aftercare options after PtDA-usage. ${ }^{9}$ However, using the PtDA during the consultation increased consultation time significantly. ${ }^{9}$ Based on the results, the PtDA was developed further to make it more attractive for health professionals to implement into clinical practice. The PtDA was adjusted so it could be used before the consultation in an effort to save actual consultation time.

It is known from the literature that many health interventions and innovations like the above-mentioned aftercare PtDA are being developed but are hardly ever used in clinical practice, not even if their relevance to the healthcare field has been shown. ${ }^{10}$ To help implement the PtDA, an effective implementation strategy is necessary, taking into account any facilitators and barriers for adoption in the healthcare setting ${ }^{11,12}$ by healthcare professionals, such as surgeons, radiation oncologists, medical oncologists, breast care nurses, nurse practitioners and physician assistants.

Fleuren, Wiefferink, and Paulussen ${ }^{11}$ developed a framework to provide an overview of determinants (facilitators and barriers) of general innovation processes in health care organizations. The framework combines determinants derived from several implementation theories and orders them into different categories. The first category contains determinants related to the innovation. Most of these determinants are derived from Roger's diffusion of innovation theory. ${ }^{13}$ Among others the determinants in this category are complexity of the innovation, and the relevance of the innovation to the implementers and users.

The second category contains determinants related to the adopter. These determinants are based for the largest part on social cognitive theories and describe the characteristics and behavior of the adopter. Determinants derived from these theories are for instance self-efficacy and attitude. ${ }^{14}$ 
The third category of the theoretical framework ${ }^{11}$ contains determinants related to the organization and socio-political context. Determinants related to the organization are related to the decision making processes in the organization. With the socio-political context, rules and regulations related to the innovation are meant. A determinant related to the organization can be for instance the necessary monetary funds to implement the innovation. A determinant related to the socio-political context can be the extent to which the innovation adheres to the national clinical guidelines. Based on this framework the Measurement Instrument for Determinants of Innovations (MIDI) ${ }^{15}$ has been developed to measure the determinants in all categories of the framework. The MIDI has been used in several implementation studies ${ }^{16-18}$ to identify determinants for implementation of health innovations in healthcare settings.

O'Donnell, Cranney ${ }^{19}$ performed research in the cancer care setting on the determinants for specific implementation of Decision Aids. The relevant determinants that O'Donnell et al. $^{19}$ and other studies ${ }^{20-22}$ identified were: relevance of the innovation (in the determinants associated with the innovation category), self-efficacy (in the determinants associated with the adopter category) and the extent to which the innovation can be incorporated into the existing care processes in the hospital especially regarding time aspects (in the determinants associated with the organization and socio-political context category). Furthermore, research ${ }^{23,24}$ has shown cosmopolitanism as an important adopter-related factor; cosmopolitan individuals are more willing to embrace changes in work methods (e.g. adopting an innovation) and looking outside of the own organization and country for ways to improve work methods. ${ }^{25}$

In summary, to facilitate implementation of the PtDA for aftercare, an implementation strategy that focusses on the most important facilitators and barriers for the adoption of the PtDA in clinical practice is necessary. These facilitators and barriers (determinants) for the intention to adopt need to be identified, since intention is the best predictor for behavior $^{16}$ and thus considered a plausible proxy measure. ${ }^{26}$ Therefore, the aim of the current study is to identify the determinants for the intention to adopt the PtDA by healthcare providers in the hospital setting, using the framework of Fleuren et al. ${ }^{11}$.

\section{Methods}

\section{Procedure and design}

A cross sectional survey study was performed across the Netherlands, except for the region in which previously the PtDA pilot study was conducted. Surgeons, medical oncologists, radiation oncologists, nurses and nurse practitioners that are involved in 
breast cancer aftercare, and who are possible adopters of the PtDA, were approached via e-mail through the research association for breast cancer professionals in the Netherlands (BOOG).

The e-mail consisted of a short explanation about the purpose and aim of this adoption study. Also, two links were included; one link to the online PtDA and one link to the online questionnaire. All adopters were asked to view the PtDA online before filling out the questionnaire as many questions were about the PtDA and its content.

Criteria for study inclusion (i.e. reviewing the PtDA using the included hyperlink and having a good understanding of the Dutch language) and exclusion (not being employed in a Dutch hospital, not conducting aftercare consultations) were explained in the e-mail. Screening questions were inserted into the online questionnaire to make sure these criteria were met. Furthermore, to reach as many relevant adopters as possible, each adopter was asked to forward the e-mail to other professionals meeting the inclusion criteria (i.e. snowball recruitment).

\section{The PtDA}

The current PtDA consists of five consecutive steps (Figure 6.1). Step one contains information about the primary treatment and potential late side-effects of these treatments as potential side-effects may influence patient's choices for an aftercare trajectory. Step two contains questions, from two validated screening inventories ${ }^{27,28}$, about problems that patients might experience after primary treatment in four domains of their life. ${ }^{29,30}$ Step three contains questions to assess the patient's preferences for characteristics related to the available aftercare options. Step four contains a brief explanation of each of the options followed by an assessment of the patient's first impression of each option. Step five provides the patients with two overviews. The first combines the patient's preferences regarding option characteristics and patient's first impression. The overview shows the extent to which each option matches the patient's preferences and first impressions. The second generates a visual discussion template ${ }^{29,30}$ with the problems experienced by the patient on the four domains of life.

Both overviews can be used during an aftercare consultation to decide patient goals and the content of the aftercare trajectory together. For example, if a patient is experiencing loss of physical condition and wants to improve this condition to be able to walk to work again, then evidence-based aftercare options are that the patient exercises her/himself or with a physical therapist or in cancer rehabilitation. 


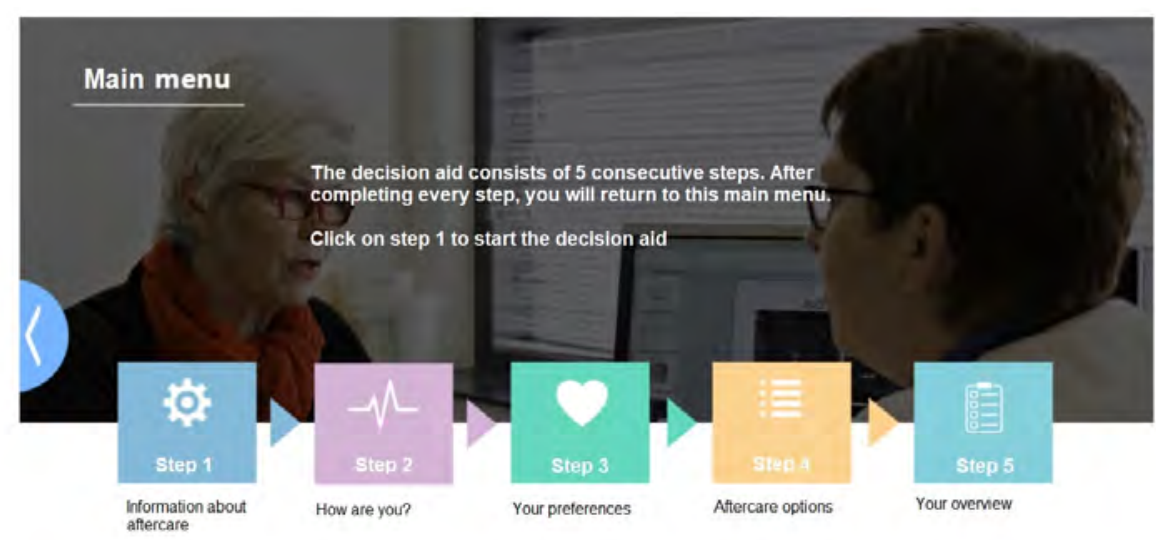

Figure 6.1 Screenshot of the main menu of the PtDA; depicting all five steps.

\section{The outcome variable}

The outcome variable in the current study was the intention to adopt the PtDA into clinical practice. A question for this study with five response categories was formulated to measure the extent to which the adopters have had the intention to adopt the PtDA: 'definitely will', 'likely will', 'neutral', 'likely will not' or 'definitely will not'. Based on their answer to this question the adopters were divided into two groups: the intenders (definitely will, likely will adopt) and the non-intenders (neutral, likely will not, definitely will not adopt).

\section{The independent variables}

Demographic characteristics were measured (age in years, employment in years, frequency of breast cancer aftercare consultations per month, profession (e.g. surgeon, nurse practitioner, radiation oncologist). The other independent variables were derived from the MIDI ${ }^{15}$ and are depicted in Table 6.1. 
Table 6.1 Determinants investigated in the current study, based on the MIDI ${ }^{15}$

\begin{tabular}{|c|c|}
\hline \multicolumn{2}{|l|}{ Determinants associated with the innovation } \\
\hline $\begin{array}{l}\text { Correctness (the extent to which the information in } \\
\text { the PtDA is correct) }\end{array}$ & $\begin{array}{l}\text { Completeness (the extent to which the information in } \\
\text { the PtDA is complete) }\end{array}$ \\
\hline $\begin{array}{l}1 \text { question with Likert-scale answer: } 1 \text { (completely } \\
\text { disagree)- } 5 \text { (completely agree) }\end{array}$ & $\begin{array}{l}1 \text { question with Likert-scale answer: } 1 \text { (completely } \\
\text { disagree)- } 5 \text { (completely agree) }\end{array}$ \\
\hline $\begin{array}{l}\text { Complexity (the extent to which the PtDA is complex } \\
\text { to use) }\end{array}$ & $\begin{array}{l}\text { Compatibility (the extent to which the PtDA is } \\
\text { compatible with the work methods) }\end{array}$ \\
\hline $\begin{array}{l}\text { Combined score from } 2 \text { questions with Likert-scale } \\
\text { answers: } 1 \text { (completely disagree)- } 5 \text { (completely agree) }\end{array}$ & $\begin{array}{l}1 \text { question with Likert-scale answer: } 1 \text { (completely } \\
\text { disagree)- } 5 \text { (completely agree) }\end{array}$ \\
\hline $\begin{array}{l}\text { Observability (the extent to which the effects of the } \\
\text { PtDA are visible) }\end{array}$ & $\begin{array}{l}\text { Relevance for the client (the extent to which the PtDA } \\
\text { is relevant for the patient) }\end{array}$ \\
\hline $\begin{array}{l}1 \text { question with Likert-scale answer: } 1 \text { (completely } \\
\text { disagree)- } 5 \text { (completely agree) }\end{array}$ & $\begin{array}{l}1 \text { question with Likert-scale answer: } 1 \text { (completely } \\
\text { disagree)- } 5 \text { (completely agree) }\end{array}$ \\
\hline
\end{tabular}

\section{Determinants associated with the adopter}

Personal benefits/drawbacks (benefits/drawbacks from PtDA-usage)

Combined score from 2 questions with Likert-scale

Outcome expectations (the outcome expectations of the adopter from working with the PtDA) answers: 1 (completely disagree)- 5 (completely agree)

Patient satisfaction (the extent to which the adopter feels the patient will be satisfied)

1 question with Likert-scale answer: 1 (completely disagree)- 5 (completely agree)

Combined score from 6 questions with Likert-scale

answers: 1 (completely disagree)- 5 (completely

agree)

Patient cooperation (the extent to which the adopter feels the patient will cooperate in working with the PtDA)

1 question with Likert-scale answer: 1 (completely disagree)- 5 (completely agree)

Social support (the extent to which the adopter expects support from co-workers)

Combined score from 2 questions with Likert-scale Descriptive norm (the extent to which the adopter expects working with the PtDA will be the norm in his/her hospital)

answers: 1 (completely disagree)- 5 (completely agree) Combined score from 2 questions with Likert-scale answers: 1 (completely disagree)- 5 (completely agree)

Subjective norm ( the extent to which the adopter Self-efficacy (the extent to which the adopter feels capable to work with the PtDA)

expects co-workers to use the PtDA)

Combined score from 5 questions with Likert-scale

Combined score from 2 questions with Likert-scale answers: 1 (completely disagree)- 5 (completely agree)

Knowledge (the extent to which the adopter has working-with-the-PtDA-knowledge)

1 question with Likert-scale answer: 1 (completely disagree)- 5 (completely agree)

answers: 1 (completely disagree)- 5 (completely agree)

Cosmopolitanism (added question) (the extent to which the adopter is open to changes in work methods)

Combined score from 4 questions with Likert-scale answers: 1 (completely disagree)- 5 (completely agree)

\begin{tabular}{|c|c|}
\hline \multicolumn{2}{|c|}{ Determinants associated with the organization/ socio- political context } \\
\hline $\begin{array}{l}\text { Financial resources (the extent to which monetary } \\
\text { funds are available for the implementation of the } \\
\text { PtDA) } \\
1 \text { question with Likert-scale answer: } 1 \text { (completely } \\
\text { disagree)- } 5 \text { (completely agree) } \\
\text { Adherence to Dutch clinical guidelines (the extent to } \\
\text { which the PtDA adheres to Dutch clinical guidelines) } \\
1 \text { question with Likert-scale answer: } 1 \text { (completely } \\
\text { disagree)- } 5 \text { (completely agree) }\end{array}$ & $\begin{array}{l}\text { Time available (the extent to which time is available } \\
\text { for the implementation of the PtDA) } \\
1 \text { question with Likert-scale answer: } 1 \text { (completely } \\
\text { disagree)- } 5 \text { (completely agree) }\end{array}$ \\
\hline
\end{tabular}

For all determinants with combined scores from multiple questions a reliability analysis was performed. For all determinants Cronbach's $\alpha>.60$. 


\section{Analyses}

For an adopter to be included in the analyses $90 \%$ or more of the questions on the questionnaire had to be completed and the adopter had to meet the inclusion criteria. Then, the background characteristics of intenders and non-intenders were compared using t-tests and chi-square tests. Results were deemed significant if $p<.05$. The background characteristics on which the two groups differed significantly were included in the logistic regressions as confounders.

To assess which specific determinants were important with regard to the adoption of the PtDA within each determinant category (i.e. innovation, adopter and organization/socialpolitical context), three logistic regressions were performed using the backward likelihood ratio procedure ( $\mathrm{Pin}=.05$; Pout=.10). The determinants that were left in the last step of each regression were then included together in one logistic regression using the one-step ENTER procedure, to identify which determinants were most important overall.

\section{Results}

\section{Sample}

In total 122 adopters participated in the study; 89 adopters had completed $90 \%$ or more of the questions, and of these 89,81 adopters met the inclusion and exclusion criteria. In total, 81 adopters were included in the analyses: 44 intenders and 37 non-intenders. Intenders and non-intenders did not differ on personal characteristics, such as age or years of employment (Table 6.2).

Table 6.2 Characteristics of the respondents in the intender and non-intender group.

\begin{tabular}{lcccc}
\hline Characteristic & $\begin{array}{c}\text { Intender group } \\
(\mathrm{N}=44)\end{array}$ & $\begin{array}{c}\text { Non-intender group } \\
(\mathrm{N}=37)\end{array}$ & F-value & p-value \\
\hline Age (years), mean (SD) & $47.34(7.56)$ & $48.46(7.99)$ & .19 & .52 \\
Employment (years), mean (SD) & $10.74(6.31)$ & $13.43(8.20)$ & 3.61 & .11 \\
Breast cancer aftercare consultations & & & & \\
per month, mean (SD) & $47.81(54.16)$ & $37.31(45.91)$ & 1.44 & .35 \\
\hline Position & \multicolumn{4}{c}{ Chi-square } \\
\hline Surgeon & 9 & 10 & & .94 \\
Medical Oncologist & 3 & 2 & & \\
Radiation oncologist & 7 & 8 & & \\
Nurse & 3 & 2 & & \\
Nurse practitioner & 15 & 10 & & \\
Other (e.g. physician assistant) & 7 & 5 & & \\
\hline
\end{tabular}




\section{Determinants of adoption}

The first backward logistic regression performed to analyse determinants associated with the PtDA resulted in two significant determinants in the fifth step (Table 6.3). Both compatibility and relevance for the patient were significantly positively associated with the intention to adopt.

The second backward logistic regression analysis on determinants associated with the adopting person resulted in two determinants in step six that were significantly positively associated with the intention to adopt. These determinants were self-efficacy and outcome expectations (Table 6.3).

The third and final backward logistic regression analysis focussed on determinants related to the organization and socio-political context and resulted in one remaining significant determinant positively related to the intention to adopt in step four. This determinant was adherence to the guidelines (Table 6.3).

All the determinants that remained in the final step across all three categories were analysed together in a logistic regression analyses with the ENTER procedure. This resulted in two significantly and positively associated determinants with the intention to adopt, which were: relevance for the patient and self-efficacy (Table 6.4).

Table 6.3 Results on the backward logistic regression analysis for determinants associated with the PtDA.

\begin{tabular}{|c|c|c|c|c|c|}
\hline & \multirow[t]{2}{*}{ OR (Odds Ratio) } & \multicolumn{2}{|c|}{ 95\% Confidence Interval } & \multirow[t]{2}{*}{$p$-value } & \multirow[t]{2}{*}{$\mathrm{R}^{2}$} \\
\hline & & Lower & Upper & & \\
\hline \multicolumn{6}{|l|}{$\begin{array}{l}\text { Step } 5 \text { of analysis of } \\
\text { determinants associated } \\
\text { with the PtDA: }\end{array}$} \\
\hline Compatibility & 2.08 & 1.14 & 3.80 & .02 & \\
\hline Relevance for the patient & 16.73 & 2.54 & 17.86 & .00 & .43 \\
\hline \multicolumn{6}{|l|}{$\begin{array}{l}\text { Step } 6 \text { of analysis of } \\
\text { determinants related to } \\
\text { the adopter: }\end{array}$} \\
\hline Outcome expectations & 48.22 & 3.18 & 731.39 & .01 & \\
\hline Social Support & 2.92 & .78 & 10.99 & .11 & \\
\hline Descriptive norm & 1.73 & .99 & 3.04 & .06 & .60 \\
\hline Subjective norm & .25 & .06 & 1.07 & .06 & \\
\hline Self-efficacy & 10.34 & 1.25 & 85.82 & .03 & \\
\hline \multicolumn{6}{|l|}{$\begin{array}{l}\text { Step } 4 \text { of analysis of } \\
\text { determinants related to } \\
\text { the organisation/ the } \\
\text { socio-political context: }\end{array}$} \\
\hline Adherence to guidelines & 3.37 & 1.42 & 8.01 & .01 & .15 \\
\hline
\end{tabular}


Table 6.4 Results on the logistic regression analysis with ENTER procedure with the most important determinants from all three determinant categories.

\begin{tabular}{|c|c|c|c|c|c|}
\hline & \multirow[t]{2}{*}{ OR (Odds Ratio) } & \multicolumn{2}{|c|}{ 95\% Confidence Interval } & \multirow[t]{2}{*}{$p$-value } & \multirow[t]{2}{*}{$\mathrm{R}^{2}$} \\
\hline & & Lower & Upper & & \\
\hline \multicolumn{6}{|l|}{ Determinants in the analysis } \\
\hline Compatibility & 1.33 & .57 & 3.12 & .51 & \\
\hline Relevance for the patient & 7.40 & 1.85 & 29.58 & .01 & \\
\hline Outcome expectations & 14.77 & .76 & 285.23 & .08 & \\
\hline Social support & 3.67 & .69 & 19.47 & .13 & .70 \\
\hline Subjective norm & .39 & .08 & 1.91 & .25 & \\
\hline Self-efficacy & 14.56 & 1.09 & 194.09 & .04 & \\
\hline Descriptive norm & 1.78 & .85 & 3.69 & .13 & \\
\hline Adherence to the guidelines & .71 & .21 & 2.37 & .57 & \\
\hline
\end{tabular}

\section{Discussion}

The current study aimed to identify determinants for the intention to adopt the PtDA by healthcare providers in the hospital setting. For determinants associated with the PtDA, compatibility and relevance for the patient had a significant positive association with the intention to adopt. For determinants associated with the adopter self-efficacy and outcome expectations were significantly related. For determinants associated with the organization and the socio-political context, only adherence to the guideline had a significant positive association with the intention to adopt. When all determinants were analyzed together only self-efficacy and relevance for the patient remained significantly associated with the intention to adopt.

A previous study ${ }^{22}$ showed that a barrier is the concern that an innovation might not have added value to the patient as the healthcare provider is more able to provide the necessary information than the PtDA. ${ }^{22}$ However, the aftercare PtDA proved relevant to personalize aftercare for curatively treated breast cancer patients in the pilot study. ${ }^{9}$ This point may have to be accentuated in the implementation strategy to increase the likelihood of adoption of the aftercare PtDA into clinical practice. Self-efficacy was also significantly associated with the intention to adopt, which is consistent with findings in previous studies examining the implementation of PtDAs into clinical practice. Therefore one of the focus points in the implementation strategy has to be increasing the selfefficacy of the adopters, e.g. by developing e-learing models.

The second significantly positively related variable with the intention to adopt in the category of determinants associated with the PtDA was compatibility. Earlier research has shown that the ability to insert the innovation into the current workflow increases the chances of implementation into clinical practice. ${ }^{22}$ This result is replicated for the 
aftercare PtDA. Furthermore, previous research indicated that usability or complexity for the patient is important. ${ }^{20-22}$ In this research this was not one of the main determinants for intention to adopt. The IPDAS checklist was developed with guidelines to take into account during the development of a PtDA. ${ }^{31}$ These guidelines recommend strategies to make information in the PtDA accessible and comprehensible, resulting in a less complex program. Both health professionals that did and did not intend to adopt the PtDA rated program complexity as low and the PtDA as comprehensible for patients. During the development of the current PtDA these IPDAS guidelines were followed possibly resulting in complexity of the PtDA not to be a possible barrier for the intention to adopt.

In the category of determinants related to the adopter, two determinants had a significant positive relationship with the intention to adopt. These determinants were outcome expectations and self-efficacy. The more positive the outcome expectations were, the higher the chance of implementation of the PtDA. It has been known from behavioural change studies since many years that outcome expectations influence intention. Researchers found the same result in a study for the implementation of a computerized lifestyle change innovation. ${ }^{32}$ In the pilot study testing the effects of the aftercare PtDA positive results were found. The aftercare PtDA increases Shared Decision Making slightly and the PtDA seems to have a positive effect on patient choice for aftercare with more patients choosing less intensive aftercare after PtDA-usage. ${ }^{9}$ Presenting these positive outcomes to adopters may influence their outcome expectations and consequently increase the chance of implementation of the aftercare PtDA.

Adherence to the guidelines was the only determinant, from the category of determinants associated with the organization and socio-political context, significantly positively associated with the intention to adopt. Therefore, in creating the implementation strategy it may be effective to stress that PtDA-usage adheres to the Dutch clinical guidelines currently in place for mamma carcinoma aftercare. A determinant in the same category is time. This determinant did not have a significant association with the intention to adopt although this was expected seen as previous research did identify time as a barrier. ${ }^{19-22,24}$ It was made clear in the current study that the PtDA was meant to be used before instead of during the consult, hence time was possibly no longer a determinant for the adoption of the PtDA. Experience with PtDAs was no explanation for the fact that time was no significant determinant in the current study, as this did not influence the intention to adopt. 


\section{Strengths and limitations}

Several strengths can be identified related to this study. Firstly, with the current research, representatives from a variety of disciplines were included (e.g. nurses, radiation oncologists, medical oncologists, surgeons), although, some of the disciplines only had a few representatives. Even though, we did not have the statistical power to assess determinants for adoption for each discipline individually, this study does provide insight into barriers and facilitators of a breast cancer aftercare PtDA as reported by a broad spectrum of health professionals breast cancer patients might encounter during their treatment and aftercare decision making.

Secondly, the analyses were performed with an intender group that consisted of adopters that had the intention to adopt the PtDA (i.e. that answered either "will probably adopt" or "will definitely adopt)"; all adopters that answered neutrally were included in the non-intender group, applying a more conservative approach to identifying determinants of adoption. However, it can potentially be a limitation as well, while it is unclear if adopters that answered neutral were more inclined to adopt or to not adopt the PtDA. If they were more inclined to adopt, the results found may not be representative. To form two groups with a comparable number of respondents, it was necessary to include the neutral answering adopters in the non-intenders group as only 9 respondents answered with "will probably not" adopt or "will definitely not adopt"; which we take as a positive sign for the adoption of the PtDA into clinical practice.

However, limitations of the study should also be noted. The study had a cross-sectional design, so no inferences about causality or predictability can be made based on these results which can be viewed as a limitation. Nevertheless, it provides an overview of barriers and limitations that are of influence at this time. Since the PtDA is meant to be implemented at this time it is not a very big limitation. To study if the found determinants in the current study were really involved in the implementation of the PtDA, further research is necessary. For instance a process evaluation study on the implementation of the PtDA could be useful to determine if the same determinants are found and at the same time provide an insight of the implementation strategy used.

\section{Conclusions}

The most important determinants to intention to adopt the PtDA were self-efficacy and relevance for the patient. Therefore, this is where the emphasis of the implementation strategy should be on. Other determinants that were positively related to the intention to adopt were compatibility, outcome expectations and adherence to the clinical guidelines. 


\section{Practice implications}

The implementation strategy should focus on increasing self-efficacy of the adopter and stressing the relevance of the PtDA for the patient. One method to increase self-efficacy is vicarious learning. It entails seeing peers using the PtDA so potential adopters feel more able to work with the PtDA themselves. In healthcare settings "champions", which are healthcare providers that promote usage of health innovations to improve work methods ${ }^{33,34}$ are already used. Seeing this "champions" work with and promote working with the PtDA, thereby modelling PtDA-usage might increase self-efficacy of the adopters and increase the likelihood of implementation. Vicarious learning can potentially also influence compatibility. ${ }^{13,35}$ The champions can teach by using the PtDA that it can be compatible or even improve current work methods.

The other focus point of the implementation strategy should be to stress the relevance of the PtDA for the patient. This might be achieved by presenting the positive results of the pilot study of the effects of the PtDA on patient choice. ${ }^{9}$ It shows that patients that used the PtDA were more likely to choose another form of aftercare than the standard option, thereby personalizing their aftercare. These results might be presented in training for the PtDA for adopters. Furthermore, a training can inform the adopters that the PtDA adheres to the current clinical guidelines which can improve implementation likelihood. Furthermore, the online training can use the results of the pilot study ${ }^{9}$ also to increase the outcome expectations of the adopters.

\section{Acknowledgements}

We would like to thank Prof. dr. C.A.J. Klaassen, emeritus at the University of Amsterdam for providing statistical advice and support. 


\section{References}

1. Klaassen L, Dirksen C, Boersma L, Hoving C, of the Bbg. Developing an aftercare decision aid: assessing health professionals' and patients' preferences. Eur J Cancer Care. 2017; e12730.

2. Integraal kankercentrum Nederland [Comprehensive Cancer Centre the Netherlands ] (IKNL). NABON Richtlijn Mammacarcinoom [NABON Guideline Mammacarcinoma]. Revised 2017. Retrieved from: https://www.oncoline.nl/index.php

3. Integraal Kankercentrum Nederland [Comprehensive Cancer Centre the Netherlands ] (IKNL). 2011. Richtlijn Herstel na Kanker [Guideline Cancer survivorship care]. Retrieved from: http://www.oncoline.nl/ herstel-na-kanker.

4. Integraal Kankercentrum Nederland [Comprehensive Cancer Centre the Netherlands] (IKNL). 2017. Richtlijn Medische Specialistische Revalidatie bij Oncologie. [Guideline Medical Specialist OncologicalRehabilitation] Retrieved from: https://richtlijnendatabase.nl/uploaded/docs/Oncologie/ RL_Medisch_Specialistische_Revalidatie_bij_Oncologie_voor_autorisatie_def.pdf

5. Stacey D, Legare F, Lewis K, Barry MJ, Bennett CL, Eden KB, et al. Decision aids for people facing health treatment or screening decisions. Cochrane Database Syst Rev. 2017;4:Cd001431.

6. Belkora J, Volz S, Loth M, Teng A, Zarin-Pass M, Moore D, et al. Coaching patients in the use of decision and communication aids: RE-AIM evaluation of a patient support program. BMC Health Serv Res. 2015;15:209.

7. Woodard TL, Hoffman AS, Crocker LC, Holman DA, Hoffman DB, Ma J, et al. Pathways: patient-centred decision counselling for women at risk of cancer-related infertility: a protocol for a comparative effectiveness cluster randomised trial. BMJ Open. 2018;8(2):e019994.

8. Quigley M, Dillon MP, Fatone S. Development of shared decision-making resources to help inform difficult healthcare decisions: An example focused on dysvascular partial foot and transtibial amputations. Prosthet Orthot Int. 2018:309364617752984.

9. Klaassen LA, Dirksen CD, Boersma LJ, Hoving C. A novel patient decision aid for aftercare in breast cancer patients: A promising tool to reduce costs by individualizing aftercare. Breast. 2018;41:144-50.

10. Greenhalgh T, Wherton J, Papoutsi C, Lynch J, Hughes G, A'Court C, et al. Beyond Adoption: A New Framework for Theorizing and Evaluating Nonadoption, Abandonment, and Challenges to the Scale-Up, Spread, and Sustainability of Health and Care Technologies. J Med Internet Res. 2017;19(11):e367.

11. Fleuren $M$, Wiefferink $K$, Paulussen $T$. Determinants of innovation within health care organizations: literature review and Delphi study. Int J Qual Health Care. 2004;16(2):107-23.

12. Baker R, Camosso-Stefinovic J, Gillies C, Shaw EJ, Cheater F, Flottorp S, et al. Tailored interventions to address determinants of practice. Cochrane Database Syst Rev. 2015(4):Cd005470.

13. Rogers EM. Diffusion of preventive innovations. Addictive Behaviors. 2002;27:989-93.

14. Ajzen I. From Intentions to Actions: A Theory of Planned Behavior. In: Kuhl J, Beckmann J, editors. Action Control: From Cognition to Behavior. Berlin, Heidelberg: Springer Berlin Heidelberg; 1985. p. 11-39.

15. Fleuren MAH, Paulussen TGWM, Van Dommelen $P$, Van Buuren S. Towards a measurement instrument for determinants of innovations. Int J Qual Health Care. 2014;26(5):501-10.

16. Kolkman DGE, Fleuren MAH, Wouters M, de Groot CJM, Rijnders MEB. Barriers and facilitators related to the uptake of four strategies to prevent neonatal early-onset group B haemolytic streptococcus disease: a qualitative study. BMC Pregnancy Childbirth. 2017;17(1):139.

17. Verberne LM, Kars MC, Schepers SA, Schouten-van Meeteren AYN, Grootenhuis MA, van Delden JJM. Barriers and facilitators to the implementation of a paediatric palliative care team. BMC Palliat Care. 2018;17(1):23.

18. Schepers SA, Sint Nicolaas SM, Haverman L, Wensing M, Schouten van Meeteren AYN, Veening MA, et al. Real-world implementation of electronic patient-reported outcomes in outpatient pediatric cancer care. Psychooncology. 2017;26(7):951-9.

19. O'Donnell S, Cranney A, Jacobsen MJ, Graham ID, O'Connor AM, Tugwell P. Understanding and overcoming the barriers of implementing patient decision aids in clinical practice. J Eval Clin Pract. 2006;12(2):174-81. 
20. Wang EH, Gross CP, Tilburt JC, Yu JB, Nguyen PL, Smaldone MC, et al. Shared decision making and use of decision AIDS for localized prostate cancer : perceptions from radiation oncologists and urologists. JAMA Intern Med. 2015;175(5):792-9.

21. Caldon LJ, Collins KA, Reed MW, Sivell S, Austoker J, Clements AM, et al. Clinicians' concerns about decision support interventions for patients facing breast cancer surgery options: understanding the challenge of implementing shared decision-making. Health Expect. 2011;14(2):133-46.

22. O'Brien MA, Charles C, Lovrics P, Wright FC, Whelan T, Simunovic M, et al. Enablers and barriers to using patient decision aids in early stage breast cancer consultations: a qualitative study of surgeons' views. Implement Sci. 2014;9:174.

23. Veldwijk J, Hoving C, van Gelder BM, Feenstra TL. Potential reach of effective smoking prevention programmes in vocational schools: determinants of school directors' intention to adopt these programmes. Public health. 2012;126(4):338-42.

24. Ellen ME, Leon G, Bouchard G, Ouimet M, Grimshaw JM, Lavis JN. Barriers, facilitators and views about next steps to implementing supports for evidence-informed decision-making in health systems: a qualitative study. Implement Sci. 2014;9:179.

25. Becker $\mathrm{MH}$. Factors affecting diffusion of innovations among health professionals. Am J Public Health. 1970;60(2):294-304.

26. Eccles MP, Hrisos S, Francis J, Kaner EF, Dickinson HO, Beyer F, et al. Do self- reported intentions predict clinicians' behaviour: a systematic review. Implement. Sci. 2006;1:28.

27. Lynch J, Goodhart F, Saunders Y, O'Connor SJ. Screening for psychological distress in patients with lung cancer: results of a clinical audit evaluating the use of the patient Distress Thermometer. Support Care Cancer. 2011;19(2):193-202.

28. Braeken AP, Lechner L, van Gils FC, Houben RM, Eekers D, Ambergen T, et al. The effectiveness of the Screening Inventory of Psychosocial Problems (SIPP) in cancer patients treated with radiotherapy: design of a cluster randomised controlled trial. BMC Cancer. 2009;9:177.

29. Lenzen SA. 2015. Het vier bollen model [The four circle model]. Retrieved from: . http://www.zuyd.nl/onderzoek/lectoraten/autonomie-en-participatie/ /media/Files/Onderzoek/ Kenniskring\%20Autonomie\%20en\%20participatie\%20van\%20chronisch\%20zieken/Het\%204\%20bollen\%20model\%20voor\%20website\%20121015.pdf

30. Friesen-Storms JHHM, Bours GJJW, Snijders ICG, van der Weijden T, Jie K-SG, Beurskens AJHM. A conversation approach based on shared goal-setting and shared decision-making for nurses in cancer aftercare: A developmental study. Eur J Oncol Nurs. 2018;35:107-16.

31. Volk RJ, Coulter A. Advancing the science of patient decision aids through reporting guidelines. BMJ Qual Saf. 2018;27(5):337-9.

32. Carlfjord S, Lindberg M, Bendtsen $\mathrm{P}$, Nilsen $\mathrm{P}$, Andersson A. Key factors influencing adoption of an innovation in primary health care: a qualitative study based on implementation theory. BMC Fam Pract. 2010;11:60.

33. Dopson S, FitzGerald L, Ferlie E, Gabbay J, Locock L. No magic targets! Changing clinical practice to become more evidence based. Health Care Manag Rev. 2010;35(1):2-12.

34. Hendy J, Barlow J. The role of the organizational champion in achieving health system change. So Sci Med. 2012;74(3):348-55.

35. Soni A, Amin A, Patel DV, Fahey N, Shah N, Phatak AG, et al. The presence of physician champions improved Kangaroo Mother Care in rural western India. Acta Paediatr. 2016;105(9):e390-5. 



\section{Chapter}

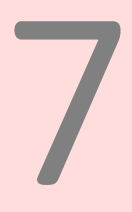

There is more to learn from stories of lymphoma survivors to further improve aftercare:

a narrative inquiry

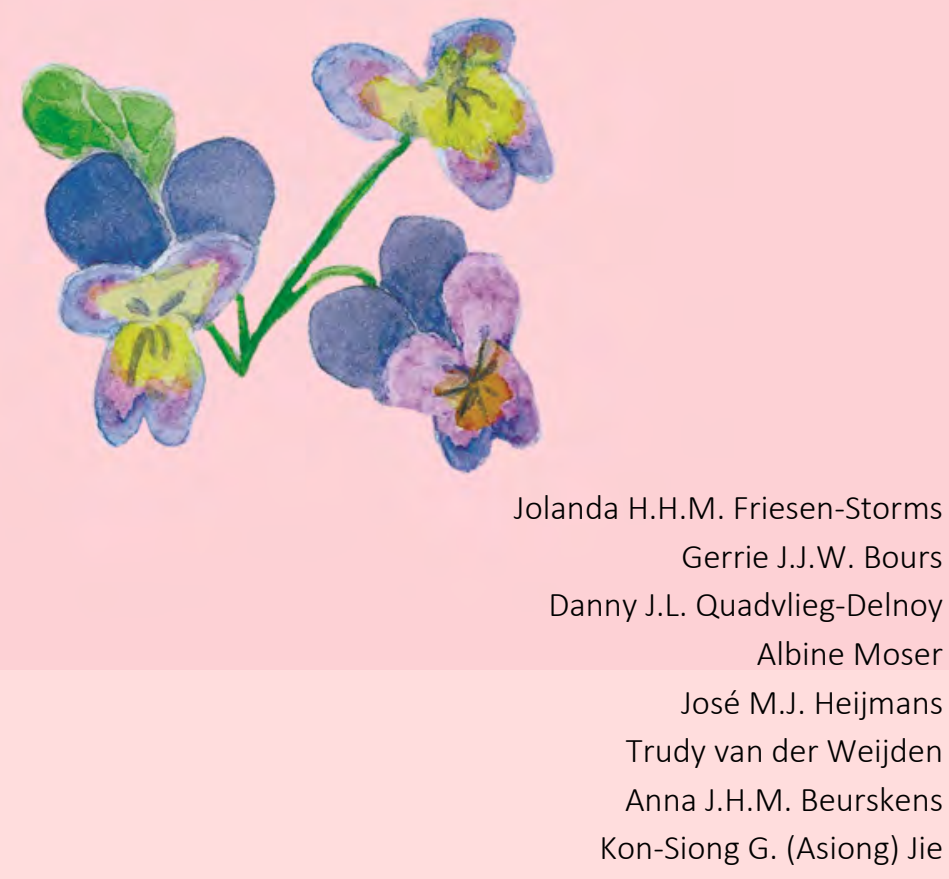




\section{Abstract}

\section{Purpose}

This study aimed to gain in-depth insight into the personal stories of lymphoma survivors in early aftercare who have been exposed to a best-practice aftercare consultation. The research question was: What are the experiences of lymphoma survivors in early lymphoma aftercare?

\section{Methods}

A narrative inquiry was conducted with lymphoma survivors in a large teaching hospital in the south of the Netherlands. They were recruited after a best-practice aftercare consultation with an advanced practice nurse.

\section{Results}

Twenty-two patients were interviewed. When analyzing the narratives, six themes emerged: living and dealing with health consequences, coping with work and financial challenges, having a positive outlook and dealing with uncertainty deriving strength from and experiencing tensions in relationships, getting through tough times in life, and receiving support from health care professionals.. Participants had to deal with health consequences like fatigue and lengthy absence from work. Their experiences and strategies for dealing with the consequences of the lymphoma varied. The social support they received was greatly valued, although some social relationships had deteriorated. The added value of the aftercare consultation and the need for further consultations was experienced differently.

\section{Conclusions}

The stories of lymphoma survivors in early aftercare revealed their experiences of how they coped with various issues in their personal lives. Addressing survivors' social support and selfmanagement capabilities, and knowing patient as a person might help to better tailor their aftercare.

Implications for practice: An aftercare trajectory based on a systematic approach might help to provide personalized aftercare. 


\section{Introduction}

Lymphomas are a heterogeneous group of hematological malignancies that arise from lymphocytes. Lymphomas are divided into two main subtypes: non-Hodgkin lymphoma (NHL) and Hodgkin lymphoma $(\mathrm{HL}){ }^{1}$ Treatment options for lymphomas include chemotherapy, radiation therapy, immunotherapy, targeted therapy and bone marrow transplantation. ${ }^{1}$ Worldwide malignant lymphomas (NHL and $\mathrm{HL}$ ) account for 3.2 percent of all cancers. ${ }^{2}$ The age-standardized incidence of $\mathrm{NHL}$ in the Netherlands is 11 per 100,000 people each year, and the 5-years survival rate varies between 37 and 85 percent dependent on the type of $\mathrm{NHL}$. The age-standardized incidence of $\mathrm{HL}$ is 2.4 per 100,000 people each year and the 5 -years survival rate is 85 percent. $^{2,3}$

Treatment of lymphomas is often associated with long-term issues like fatigue, physical activity deficits, relationship concerns, emotional and cognitive consequences, and financial, employment, and insurance issues. ${ }^{4-11}$ These issues may reduce the quality of life of these hematological oncology patients. ${ }^{12}$ Advances in treatment efficacy have improved cure and remission rates, as a result of which more survivors are living with long-term issues of lymphoma treatment. ${ }^{13}$ In addition, the transition period, just after completion of therapy, might cause distress, and lymphoma survivors may have unmet needs. ${ }^{4,14-16}$ Lingering physical effects of treatment (e.g. fatigue), more time to reflect on fear of recurrence, and decreased contact and support from the health team after treatment may all contribute to distress. ${ }^{15}$

Few cancer survivors receive comprehensive aftercare, although potential issues and needs have been identified for which evidence-based guidelines exist. ${ }^{17,18}$ Studies of the burden of symptoms have traditionally focused primarily on examining the symptom burden among patients with solid tumors ${ }^{19}$ and the Dutch guideline on aftercare ${ }^{17}$ also focuses on curable cancer patients with solid tumors. Although the Dutch aftercare guideline can be used for hematological patients, their individualized aftercare in routine practice may be different. ${ }^{19}$ Addressing issues and needs by providing patient-centered evidence-based aftercare in an early stage after treatment might help patients reach a "new normal". 8,9,16,20,21 Guidelines recommend a systematic approach to aftercare, based on shared decision-making, in which patients' issues, goals, and wishes are systematically screened, shared, and integrated in an individualized cancer survivorship plan. ${ }^{17,22,23}$

Research into patients' experience of aftercare management may inform and enable health care professionals to deliver better, tailored, patient-centered, evidence-based aftercare. ${ }^{24}$ Furthermore, it is important to understand which matters are important to cancer survivors, and which care planning strategies are consistent with their issues and needs, in order to optimize aftercare planning. ${ }^{25}$ 
Although some research has addressed lymphoma survivors' aftercare needs, ${ }^{21,26,27}$ there have been few in-depth qualitative studies addressing their experience of aftercare. Some qualitative studies $8,27,28$ have conducted interviews (including focus group interviews) to explore post-treatment experiences and preferences for follow-up, or to investigate what the lived experiences of survivors of acute leukemia and highly malignant lymphoma mean to them. ${ }^{28}$ These studies were all conducted more than three months after treatment, with average intervals ranging from 14.6 to 29 months or even two years or more. Survivors included in the studies do not seem to have been provided with a systematic aftercare consultation soon after completion of treatment. There is a lack of evidence from studies using a narrative approach to evaluate in-depth the experiences of lymphoma survivors in early aftercare who are exposed to a bestpractice systematic aftercare consultation in which they are stimulated to think about their issues, goals, and possible aftercare options. The personal stories of patients are a powerful source for a rich description of experiences of patients and the meanings that the participants derive from their experiences. ${ }^{29}$ Narratives provide a "window" into the way individuals story their experiences within the context of their wider lives. ${ }^{30}$ Furthermore, narratives invite us to enter the perspective of the narrator and might induce health care professionals to take action. ${ }^{30}$

The aim of the current study was to examine in-depth the personal stories of lymphoma survivors in early aftercare who have been exposed to a best-practice aftercare consultation. The research question was: What are the experiences of lymphoma survivors in early lymphoma aftercare ? The results can be used to further improve early lymphoma aftercare and to provide patients with more tailored and coordinated aftercare. $^{25}$

\section{Methods}

The method used was that of a qualitative narrative inquiry. ${ }^{30}$ The rationale for using a qualitative narrative inquiry is to obtain an in-depth understanding of the experiences of lymphoma survivors, and the meaning that they give to these experiences of survivorship in the context of aftercare. Narrative inquiry was the appropriate design as we wanted to investigate "real-life problems." Narratives contain the, rich, detailed and often personal perspectives. Narrative inquiry emphasizes the story, typically including both what is narrated and how it is told. Open-ended questions that open up topics and allow respondents to construct answers in ways they find meaningful are more likely to provide narrative opportunities. ${ }^{30}$ The emotional attentiveness and engagement, and 
the degree of reciprocity in the conversation is more important than the exact formulation of the questions.

\section{Setting}

The setting was a malignant lymphoma care pathway in a large, urban 600-bed teaching hospital in the south of the Netherlands. About 48 patients are treated for malignant lymphoma within this care pathway each year. Three hematologists have the medical responsibility for the patients, and one advanced practice nurse (APN) supports these patient during their disease trajectory.

Starting from March 2017, all curatively treated lymphoma patients were invited by the APN for an aftercare consultation, based on a systematic conversation approach. This conversation approach integrates shared decision making with evidence-based practice $^{31}$, and addresses patient goals. The approach consists of four phases ${ }^{32}:$ (1) preparing the aftercare consultation; (2) shared goal setting; (3) shared care planning; and (4) evaluation. We incorporated practical tools within the different phases of the approach. $^{32}$

The APN had attended a training course on the conversation approach. The consultation was incorporated into routine care. From March till June 2017, the APN applied the model in routine practice and received in-service coaching.

\section{Sample}

The sample for this study was recruited between June 2017 and March 2018, and consisted of 33 people who were exposed to the aftercare consultation approach.

Inclusion criteria were: (1) having been diagnosed with NHL or HL; (2) having been treated with chemotherapy, whether or not combined with other therapy; (3) being in remission.

Patients were excluded if they had insufficient command of Dutch.

\section{Sampling procedures}

The APN invited patients to participate in our study after they had had their aftercare consultation, which was conducted between three and eight weeks after treatment. Patients who agreed to participate received written information and were contacted by telephone. All interviewed patients provided written informed consent. Patients were interviewed within 3 weeks after the consultation, so they were still able to remember the consultation. 
After having included the first 17 consecutive patients, the APN purposively sampled the next group of patients to maximize variation, selecting patients of younger age and/or with a more chronic type of lymphoma and/or with multiple problems. We defined data saturation within our study as the point at which no new themes emerged and where the themes across respondents showed a repetition of similar responses in the unique narratives. ${ }^{33}$ This data saturation was reached after 17 interviews. We nevertheless included some more interviews. This was done, firstly, to confirm saturation, ${ }^{33}$ and secondly to obtain additional narrative details within the themes.

\section{Data and data collection}

We collected data by narrative interviewing. We developed an interview guide with open questions, as is suggested in the literature. ${ }^{30}$ We first invited respondents to tell about their experiences of early aftercare in personal stories. Examples of questions asked were: How are you doing now ? How did you resume your everyday life after you received the diagnosis and treatment? We then asked questions about their experiences with the care they had received. Subsequently, we further discussed certain topics like the main consequences of the disease and treatment and what they did to recover from the consequences of the disease. The interviews created opportunities for extended narration, and we used the interview guide very flexibly by following the respondents' stories. The interviews mostly lasted between 45 and 90 minutes, although three interviews were shorter because the patients did not feel like spending much time.

\section{Data analysis}

The interviews with the patients were audiotaped and transcribed verbatim. The transcripts were read several times before and during the analysis, which was facilitated by using NVivo11 software. We conducted a thematic narrative analysis as described by Riessman. ${ }^{30}$ Two researchers (JF and JH) independently analyzed a total of five interviews and reflected on their content and the themes that emerged in two analytical sessions. In addition, the primary researcher (JF) planned two analytic sessions with a researcher (AM) who is an expert in qualitative research, to discuss the themes. The primary researcher then coded all the interviews according to the themes the researchers had agreed upon. In the next phase, she planned an analytic session with the expert (AM) to reflect on ways to present the themes in such a way as to do justice to the "wholeness" of the narratives provided by participants. The primary researcher then further fine-tuned the themes within the results section and discussed the themes within the results section in the research team (JF, GB, AB, KJ, TW). The researcher (JH) who had independently coded the first five interviews read all remaining interviews, and 
in a final session she and the primary researcher (JF) agreed on the how the themes were presented within the results section.

\section{Trustworthiness}

We used several strategies to ensure trustworthiness, in terms of credibility, transferability, dependability, and confirmability. ${ }^{34,35}$ Investigator triangulation was used by involving several researchers in a research team in the data collection and analysis (see above), as well as involving them in addressing the organizational aspects of the study. Some of the researchers were clinical practitioners and were familiar with the study setting. We promoted transferability of the knowledge gained in this study to other contexts by providing a "thick description" of the participants and the research process. To ensure dependability, we used the theory for narrative thematic analysis ${ }^{30}$ and made sure our study conformed to the quality standards for Reporting Qualitative Research. ${ }^{36}$ To ensure confirmability, the primary researcher (JF) discussed and reflected on the study design and analysis with a researcher who is an expert in qualitative research (AM). In narrative research, utility is often mentioned as a quality criterion. ${ }^{30}$ We have formulated recommendations ensuing from our findings, which can be used to further improve early aftercare.

\section{Results}

Twenty-two cancer survivors with NHL or HL participated in this study. Of the 31 eligible patients, 9 patients declined to participate. Reasons for not participating included patients wanting close the illness period $(n=4)$ and patients being too busy, for example because they were starting rehabilitation $(n=3)$. Two did not provide a reason $(n=2)$. Respondents were aged between 27 and 74 years, with a median of 65.5 years and an Interquartile Range (IQR) of 11 years. Twenty-one patients had been diagnosed with non-Hodgkin lymphoma and one patient with Hodgkin lymphoma. Fourteen patients were male and eight female. Five patients had a low educational level, eight patients an intermediate level and nine a high educational level. All patient lived in the Netherlands, 19 were of Dutch descent, one patient was of German, one of Turkish and one of Moroccan origin. All patients had sufficient command of Dutch. Four patients had had two or more recurrences of their disease. 


\section{Themes}

After analysis of the narratives, the following six relevant themes in the patients' narratives were identified: living and dealing with health consequences, coping with work and financial challenges, having a positive outlook and dealing with uncertainty, deriving strength from and experiencing tensions in relationships, getting through tough times in life, and receiving support from health care professionals. Each theme was subdivided into subthemes. (See Figure 7.1).

\section{Living and dealing with health consequences}

Fatigue, loss of physical condition and strength

All respondents still suffered from fatigue and/or loss of physical condition and strength. These were major issues. The respondents reported that they were gradually expanding their daily activities. Some had had setbacks such as inability to walk, probably due to polyneuropathy of the feet and painful muscle cramps or having weak legs. They were improving their physical condition by exercising, often resuming types of exercise they had engaged in before their illness. They had not yet regained the exercise capacity they used to have before.

The respondents' stories showed that some activities were important to them. They felt joy when they were able to resume them or longed to be able to do so. One respondent, was able to resume what was for her a meaningful activity, babysitting her grandchild. Another woman found it important that she was able to have a small birthday party again. Respondents also indicated that it was very important to them to be able to go walking or cycling in the countryside again, or to work in their garden again. These were things that they derived strength from. Or they wanted to resume a hobby like playing golf or singing in a choir.

73-year-old man diagnosed with NHL, who felt well during treatment. He is not a very talkative person. His partner was present during the interview.

'[Wife] Alone. He prefers to cycle on his own, and then it's ...

[Participant] Into the woods and then I can hear the birds singing. That's the best medicine for me.

[Wife] Cos if he has to sit inside, it drives him crazy.'

Cognitive consequences, memory loss and inability to concentrate

Some patients still had cognitive problems like memory loss and inability to concentrate. Some were not able to read a book or follow a television program. To ameliorate this, they often played memory training games or they trained their memory by working. 
Hair loss, weight loss, nerve sensations, mucosal and voice problems Physical problems the respondents were suffering from, albeit to a decreasing degree, included hair loss, weight loss, and tingling sensations and numbness in their fingers or toes, changes in defecation patterns, dry mouth or fungal infections or blisters in their mouth, dry eyes or a weak voice. Most respondents' hair had started to grow back, but they were still often wearing a wig or a headscarf. Most of the female respondents reported that the hair loss had been an emotional burden to them, and sometimes also to their families. But some of the male respondents also said that losing their hair, pubic hair and beard had been difficult for them.

\section{Coping with work and financial challenges}

\section{Coping with work}

Some of the respondents were retired, so this theme was less important to them. The stories told by those in employment varied considerably. Some had not yet resumed work, while others were working part-time and one respondent was working at the same level as before he became ill. He kept working during his treatment, though making fewer hours and at a slower rate. The respondents had made agreements with their employers about the way they intended to resume their job. Some indicated that their employer and/or the colleagues at work were very considerate about their illness.

58-year-old man diagnosed with a chronic form of NHL, who felt fairly well during treatment.

'Yeah, I have a nice job and I'm also very pleased with the way my employer is currently dealing with my illness. I'm being given all the space, all the freedom I need to arrange things my way. When I started the chemotherapy I immediately got an email from the person who took over my work and takes care of my work for me, so that was perfectly arranged. Yeah, right, right, yeah, exactly, so yeah, nice job still. I'm hoping to soon resume the work fully, as it involves quite a bit of traveling.'

Respondents often mentioned that resuming their job was important to them. One selfemployed respondent had started working as much as possible again, as this was also a form of therapy for him. In some cases the illness led to problems with the respondent's employment contract, for instance, a temporary contract not being renewed due to the illness, or being dismissed after the illness. This led to worries about finding a new job, and finding out their legal rights. 


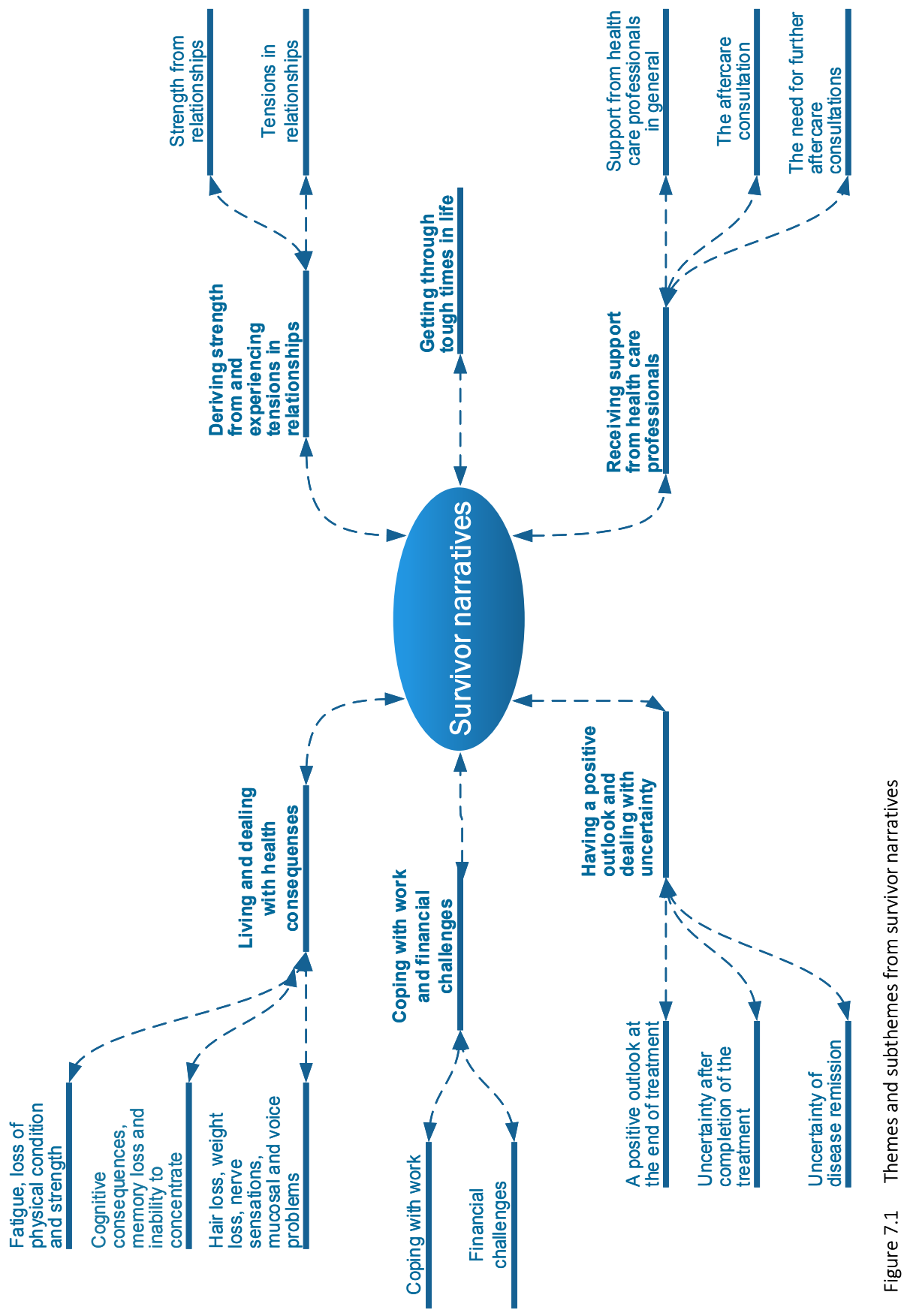




\section{Financial challenges}

Loss of work might negatively affect the respondents' financial situation. Financial considerations regarding aftercare included whether the intervention would be reimbursed by the insurance. In some cases, patients had to pay the care provider up front and then reclaim the expenses afterwards, while in other situations the provider was paid directly by the insurer. Some respondents reported that additional costs like taxi fares had made it harder for them to make ends meet, while others had sufficient financial means and less worries.

\section{Having a positive outlook and dealing with uncertainty}

\section{A positive outlook at the end of treatment}

Those respondents who were diagnosed with lymphoma for the first time and had received favorable test results after the treatment were hopeful that the disease would not return. They had often gone through a period of uncertainty before the diagnosis and had courageously undergone the treatment. They were often relieved to be still alive, especially if they had known people who had died shortly after being diagnosed with cancer. Respondents had faith in their doctor when she or he said that their cancer was well treatable. They also saw the end of the treatment as the conclusion of a period and the start of a new period, in which they would work on their further recovery. Some respondents made new plans for the future, for instance having children in the future or starting to live together with a girlfriend. Some respondents also wanted to leave the period of treatment behind, and no longer wanted to dwell on it too much.

60-year-old man diagnosed with NHL, who had been admitted to hospital with a pulmonary

embolism. He was fond of sports and used to be in very good physical condition.

'Whereas, er, when I meet people I can tell them briefly how I'm doing and then say let's do something enjoyable and fun, and let's talk about things that have nothing to do with it, right? Cos I don't intend to die yet. Yeah, not thinking about it, that's, that's the main thing. Yeah, not thinking about it. At least that's my view. Anything that stops you from thinking about it increases your quality of life.'

Respondents had learned how to cope with certain issues more easily, and to set different priorities, or they tried to live by the day and take things as they come.

66-year-old woman diagnosed with NHL, who had been very weak during treatment and had been admitted to hospital with infections several times. 
'Cos he explained to me what the benefit was. That was just, should it come back, it will come back later. And I just, I just have a very positive attitude. With me the glass is always half full, and not half empty. In my case it just won't come back. Period. So, like, that's just my assumption. And, and, and well if it should come back, okay then so be it. If it happens I'll just face it as something new that happens to you. Cos to be honest I've not shed any tears over it. I just felt like well, it happens to you and there are so many people who develop cancer. Well, it's not the end of the world is it?'

\section{Uncertainty after completion of the treatment}

Some respondents had to get used to the fact that there was now 'a void', especially after the intense period of treatment with chemotherapy and check-ups at the hospital. Some of them also needed to regain trust in their own body after the intense treatment.

27- year-old woman who had been diagnosed with HL for the second time in two years.

'Well, it's, yeah, I think that's difficult, as I always had a, something to look forward to, l'd be looking forward eagerly to now, the time l'd have it all behind me, and that everything would be okay, etc. And now I get this feeling like now, there's now nothing to look forward to, and that's kind of a strange feeling. It also seems as if it's only now, well, as if I'm only now realizing all that's happened.'

\section{Uncertainty of disease remission}

Sometimes the scan did not decisively show that the disease had resolved, and patients had to return for a new scan. Sometimes patients were still on maintenance treatment, involving a new course of chemotherapy every three months over a two-year period. This could cause uncertainty. The respondents dealt with the uncertainty after treatment or the uncertainty of disease remission as part of their live. Some respondents received support from a health care professional.

The respondents who had been treated after a recurrence, and who had a more chronic form of lymphoma, were glad about favorable test results, but did still worry about the disease having recurred, and that this might happen again. One patient said that, in view of his diagnosis, he felt that each new chemotherapy 'bought him some more time'.

Patients had reacted in different ways to the possibility that the disease might recur. Reactions ranged from wanting to grasp any option, any treatment available, to not knowing what to choose in that situation because of quality of life issues, and to not wanting any further treatment or planning euthanasia with their family doctor. 
58-year-old man diagnosed with a more chronic form of NHL, who felt fairly well during treatment.

'I don't hope so. I don't assume it will, cos I actually enjoy life very much. I don't want to keel over tomorrow, but if that should be the case in the end, then, well, I didn't like this chemotherapy, so suppose I was told next, in two years' time, another chemotherapy, but six times as tough as what you've had, and that might perhaps add 6 more months to your life, then yes, I don't know what the answer will be, maybe I'll then say, like, well don't bother, and then I'll keel over tomorrow, so to speak, cos I think quality of life is also important, and well, just doing the things you like in life, that keep you on your feet.'

Some respondents reported not to be afraid of death, because of their religious beliefs, or because they had signed a euthanasia declaration or felt they had led a 'rich life'.

\section{Deriving strength from and experiencing tensions in relationships}

\section{Strength from relationships}

Respondents derived much strength and support from the assistance they received from others. This assistance took the form of people being willing to help in all kinds of ways, e.g. taking over tasks, but also providing emotional support, and being willing to listen to their story. For some, support was provided also by relatives, friends, neighbors or fellow patients. The most important support was by their partners, who were always prepared to help. The disease made some couples realize they could never live without each other, and how good it was to have a partner who could help them get through the process of illness. Some respondents talked about how good a team they were. Although the partner might sometimes have a different way of coping with the disease, e.g. by wanting to know about the details, they would remain attentive to the other's wishes.

72-year-old man diagnosed with a chronic form of NHL and who had been treated for the third time in six years.

'Yeah, that's what I liked very much too. That at a certain moment, while I was at the physical therapist's, they'd called and my wife had taken the call. And yeah, a partner can of course explain much better how the patient is doing than the patient himself, cos he tends to make things sound better than they really are.' 


\section{Tensions in relationships}

In some cases, the disease and its treatment had led to tensions in the relationship, between partners as well as with the children. They can have a different way of coping, which can result in tensions between them or can have a negative effect on the respondent's disease process, such as having more feelings of uncertainty. Some respondents had been irritable during the treatment, and had often taken it out on their partners. Some had sought counseling from a social worker or psychologist because of the tensions in their relationship. Some had solved the problems themselves, or had come to realize that they needed to understand their partner's views, or just continued their relationship as it had always been. One couple reported that they had had problems regarding intimacy and sexuality during the treatment, and that they were now in the process of rediscovering each other.

56-year-old man diagnosed with NHL, who reported that the diagnoses and disease had an impact on the well-being of the whole family. His partner was present during the interview.

'[Talking to partner] You've also had a tough time, not just me.

[JF] The whole family is involved...

Absolutely. Even after you've had your first chemo, you're not allowed to, so to speak, just kiss each other, you have to sleep in separate beds, sheets and clothes have to be kept separate, you have to go to separate toilets, and all of that is also difficult for them, right, and then if I'm troublesome as well.

They would always use the upstairs toilet, and I the downstairs one, those kind of things, right?

But fortunately it's all over now and we can start to rebuild.'

Some respondents had lost contact with friends, people who no longer visited them. The explanation they gave for this was that people did not know how to deal with the situation, 'They think cancer is contagious', and that people often had other things to do during the daytime. But some had gained new friends, or had heard from people they had not heard from for a long time, and had not expected to get in touch.

They also reported that it was easier to tell their story to some friends than to others, as some people's responses were not helpful at the time. Some respondents felt overburdened by their social contacts. They sometimes felt they could not say no or felt that they had to do something in return as the other person was also in a difficult situation. Or the people around them failed to understand why they were able to do something one day and not the next. One respondent regularly took his wife out to a camping site, to enjoy themselves and find some peace and quiet. 


\section{Getting through tough times in life}

Respondents said that apart from the treatment, life events relating to the cancer treatment or their life in general had caused them tough times and were part of their life stories. The meanings respondents assigned to this were diverse. Some respondents had faced several issues simultaneously, which was perceived as a heavy burden. Some respondents suffered from severe multimorbidity, or their partner or relatives suffered from various diseases and had even died, which in some cases had occurred just before or during the diagnosis and treatment.

60-year-old woman diagnosed with $\mathrm{NHL}$, who had been very ill during treatment and had been admitted to hospital with pneumonia, and had received a blood transfusion. Her partner was present during the interview.

'No, not at all, as my brother had just died, three weeks before. All I did was cry. They (the nurses) used to ask me why I was crying so much. And I would say I'm sad.

[JF] So you had that as well.

[Husband] Yeah, that as well. We went to the funeral. All the way from here to there. It was all too much for her. But well, there was no cure for him.'

Others said that some life events had influenced the way they dealt with their disease, for example regarding it as their duty to be active all the time or facing up to the challenges of life. Some respondents said it was important that health care professionals were aware of their issues, and not only of their cancer, and that they knew them more personally. This influenced the way they dealt with the cancer and the help they needed from health care professionals.

\section{Receiving support from health care professionals}

Support from health care professionals in general

The patients had been supported by multiple care professionals throughout the different stages. In the early aftercare phase, some of the respondents had been treated by a physical therapist, had started, or planned to start, an intake procedure for cancer rehabilitation, or had seen a psychologist or a social health worker. They mentioned a number of important components of support from health care professionals. One aspect was the continuity of care by various doctors. The respondents often placed great trust in their oncologist. They also appreciated it when their family doctor visited them proactively. Some respondents mentioned the importance of sufficient information by 
doctors. One patient felt he had had insufficient information about the nature of his disease and had looked it up on the Internet. Respondents greatly valued personal attention and empathy, like the APN who would contact them by phone after each course of chemotherapy. Some patients had had problems that they had kept to themselves which had been picked up by the APN during the phone calls. However, two patients did not appreciate being phoned. For one patient counseling by the family doctor was sufficient and the other patient mentioned that the phone calls confronted him with his disease while he was going about his usual business at home. He would have preferred appointments at the hospital.

\section{The aftercare consultation}

All of the respondents had had an aftercare consultation, based on the systematic aftercare approach, with the APN. They had mostly appreciated this consultation, and some said that it had provided added value.

65-year-old woman diagnosed with NHL, who had felt very ill and had been hospitalized for infections during chemotherapy.

'Yeah so that (the aftercare consultation) made me, er, decide to start working on it to, er, become a bit stronger. Yeah. So that's when it all started, with the massages and all. I decided I need to work on it.'

In some cases the consultation had led to additional interventions, while in other cases they had perceived it as rounding off the treatment process. Those who had had few problems or whose problems had already been addressed, attached less value to the consultation. One patient mentioned that he would have liked to have had more information about the consultation beforehand, as he had not known whether to expect a medical or a psychosocial consultation. But none of the respondents regarded the aftercare consultation as useless. They reported that the nurse had informed them about possible consequences of their disease and the various options for aftercare, like psychological help or peer support. Even if they did not feel the need for it at that time, at least they knew what was available should they feel the need for it later. Some said they were glad to have had the aftercare consultation, as it would otherwise have been too abrupt an end to the treatment process.

56-year-old man diagnosed with non-Hodgkin lymphoma, who was fond of sports, but whose physical condition had deteriorated greatly. His partner was present during the interview.

'What I must say is that the last time we saw the doctor, so you come back and you get the test results, you talk with the doctor a bit, and then he asks you would you like to do this or that, I said I just want to conclude it, it's 
over now, right? Yeah, so you get that, and then you go home, and he says I'll see you in three months, and then you come home and you think so this was it, and the way it ends. So you think he does not care about it, but then after a couple of days XXXX phoned me, and she said we're going to have and aftercare consultation, cos I first thought when the doctor said it's over, and then they more or less leave you on your own, that's what it feels like, right, and then when she phoned I thought well, I'll get a talk about what happens now.'

During the aftercare consultation, the patients set a wide variety of personal goals. One goal which was often mentioned was to regain their strength and to improve their physical condition.

According to the respondents, the aftercare consultation frequently resulted in initiatives for interventions, like oncological rehabilitation or oncological physical therapy, social work or psychological therapy. Some respondents opted to join a fitness center or to work on their physical condition on their own. Sometimes interventions discussed during the treatment phase were reviewed again during the aftercare consultation.

The arguments people used to decide whether to opt for oncological rehabilitation, a fitness center or training on their own included financial aspects, the distance to the facility, preferences regarding training in a group or alone, the freedom to attend the intervention or not, and the multidisciplinary approach of rehabilitation, as the patient had multiple problems which would be addressed in the rehabilitation program, like relationships and resuming their job.

67-year-old woman diagnosed with NHL who had been treated for the second time. She had been ill during the first three months of the most recent treatment

'Well, er, I actually decided to go for oncological physical therapy. And the address was in the southern part of town. So that was small-scale, and that's what I wanted at first. But then I thought, just at the end of the street here is the hospital, where they do oncological rehabilitation. So I then opted for the hospital, as that is closest. And I think the care there will be a bit more comprehensive. I got all the forms and so it's been applied for, and, I have to inquire at the hospital, or er, at the health insurance, to see if it's covered by my insurance or not.' 
The need for further aftercare consultations

The respondents differed in terms of whether they still felt the need for more consultations in the year after their treatment. Some wanted to end the hospital process, indicating that they had other people to fall back on, like their family doctor. Their need for a further consultation would also depend on whether they would experience any symptoms later. It would be fine if they could just have consultations over the phone with the nurse when needed. However, participants admitted that they might be more inclined to talk about certain problems in a face-to-face consultation. Other respondents preferred to have further consultations at the hospital if their family doctor was not very pro-active and might not have the necessary expertise. Another respondents would appreciate it if problems and goals were followed up in further consultations.

\section{Discussion}

\section{Summary of main findings}

This study explored the post treatment experiences of lymphoma survivors in early aftercare who were exposed to a best-practice aftercare consultation. During our study we identified six themes regarding the way the lymphoma survivors coped with health consequences, work and financial challenges, optimism and uncertainty, strengths and tensions in relationships, and additional difficult experiences in life on top of the illness experience, as well as the way they experienced support from health care professionals.

We identified the same issues that have been described in the literature, ${ }^{4,5,7-11}$ but have gained additional insights into the way lymphoma survivors deal with these issues, as part of their daily lives in early aftercare, and which care planning strategies might support them. These insights might help to further develop strategies for aftercare.

\section{Results in context}

The experiences that survivors described in the context of the various themes identified in our study are partly covered by existing frameworks like the International Classification of Functioning, Disability and $\mathrm{Health}^{37}$ or the Framework of Positive Health. ${ }^{38}$ For example, the 'My Health' domain of life, representing the patient's experiences of their health and health care problems, might be said to correspond to the theme of 'living and dealing with health consequences'. By contrast, the theme of 'receiving support from health care professionals' seems to be one that is difficult to fit within existing frameworks. 
Survivors in our study made efforts to self-manage their lives, for example by resuming activities they used to do before the disease, like walking, cycling or occupational activities. They often dealt with other life events in addition to the disease, which are also part of their life story. Most survivors had a positive outlook, though sometimes experiencing uncertainty about the disease remission. Other studies have also described how cancer survivors make the best of their lives but with an underlying fear of recurrence..$^{8,39}$ In addition, a literature review ${ }^{21}$ described fear of recurrence as a major issue, which is important for health care professionals to address.

Some survivors in our study wanted to leave the period of treatment behind them, and no longer wanted to dwell on it too much. Other studies ${ }^{8}$ confirmed that moving away from the cancer experience might help in the transition to the survivorship phase.

Social support from other people was greatly valued by our respondents, although some social relationships had deteriorated during the disease process, and some relationships were not always supportive to the respondents. Our study, like other studies, 8,40 confirms the importance of health care professionals addressing relationships, e.g. by involving the partner or other people who can provide the survivor with social support in the dialogue with them.

It is important to look at survivors from a holistic viewpoint and address their social support and self-management capabilities, in order to provide them with tailored personalized aftercare. This is confirmed by the findings of other studies ${ }^{38,41-44}$ and by evidence-based clinical aftercare guidelines. ${ }^{17,23}$ Personalized care planning based on goal-setting and shared decision-making, which is integrated in our conversation-based aftercare approach, has been shown to have positive effects on self-management capability. ${ }^{45}$ The aftercare consultation gave the survivors the opportunity to talk about their transition from active treatment to the survivorship phase, and about their issues, goals and the various options for aftercare, which sometimes led to the initiation of aftercare interventions. Some survivors in our study reported that feelings of abandonment had been prevented by the early aftercare consultation. In other studies, ${ }^{8,27}$ in which patients were not exposed to aftercare consultations, patients did indeed express feelings of being abandoned or not being understood holistically, as well as reporting a lack of information about aftercare. These studies confirmed that it might be valuable to implement aftercare consultations in routine cancer care. In addition, several studies ${ }^{15,46,47}$ suggested the need for increased structure and improved communication in survivorship-care consultations.

Our respondents varied as regards their need for further aftercare consultations. Some would prefer to receive further aftercare from their family doctor. Setting up aftercare models shared between oncological hospital care and primary care might be valuable, as has been suggested in other research. ${ }^{17,22,48} \mathrm{~A}$ web-based patient decision aid for 
choosing an aftercare trajectory ${ }^{49}$ might facilitate further tailoring of the aftercare. Some studies $^{50,51}$ showed that survivors who were allowed to choose a personalized aftercare trajectory that best suited their preferences were more satisfied with their aftercare than those who were not given a choice. In addition, decision aids improve people's knowledge about options, and reduce their decisional conflict due to feeling uninformed and unclear about personal values. ${ }^{52}$

\section{Strengths and limitations}

The narrative design of our study enabled us to obtain an in-depth description of the experiences of lymphoma survivors and the meaning they give to it in the context of their lives in early aftercare, as it was their story which guided the interview, and not a pre-structured interview guide. We deliberately included a group of patients who were exposed to a best-practice aftercare consultation approach. The narrative study gave us more in-depth insights into patients' experiences within this aftercare trajectory, especially regarding the various approaches to dealing with consequences of their lymphoma in daily life.

A limitation of our study might be selection bias, as we mainly included older patients, and younger patients might have different experiences. In a study by Persson and

Halberg, ${ }^{28}$ younger survivors of acute leukemia or highly malignant lymphoma evaluated their situation more favorably and felt that they had their whole life ahead of them.

Another limitation might be that our patients were recruited from one lymphoma care pathway, provided by only one APN who conducted the aftercare consultations. Thus, the views of the survivors in our sample represent a specific group. Although our study has revealed the experiences of survivors who were exposed to a best-practice aftercare consultation, the wider credibility of our findings needs to be established in other settings.

\section{Conclusions}

The stories of lymphoma survivors in early aftercare provided us with more in-depth insights into their experiences of coping with various issues in their personal lives. Exploring and addressing their social support and self-management capabilities, and knowing the patients as a person might help to better tailor the aftercare to them. Choosing an aftercare trajectory based on a systematic conversation based approach in which patients are stimulated to think about their issues, goals and possible aftercare options might be useful in helping patients with the transition from the treatment phase to the survivorship phase. 


\section{Implications for practice and further research}

Our study has several implications for practice and research. The first implication is that survivors' social support and self-management capabilities are important aspects to be addressed in aftercare, in order to tailor it to their individual needs, preferences and context. A systematic conversation approach involving shared goal-setting and shared care planning might help nurses and other health care professionals to provide patients with personalized aftercare. Tailoring in aftercare could be supported by decision aids that help in choosing an aftercare trajectory fitting the survivors' needs and preferences. The second implication is that it seems important to offer survivors more comprehensive aftercare support than just one aftercare consultation at the hospital, during which they can choose further aftercare options tailored to their needs and preferences. In addition, the aftercare approach we studied could also be implemented in primary care. Models for further aftercare planning involving both hospital care and primary care should be developed.

Further research in other cancer aftercare settings in which survivors are provided with an aftercare trajectory, based on the conversation approach, supported by patient decision aids, is needed to study the effectiveness of the approach. 


\section{References}

1. In: Kaushansky K, Lichtman MA, Prchal JT, Levi MM, Press OW, Burns LJ, et al., editors. Williams Hematology. Part XI: Malignant Lymphoid Diseases. 9th ed. New York: McGraw Hill Education. 2016.

2 International Agency for Research on Cancer. GLOBOCAN. 2012v1.1 Cancer Incidence and Mortality Worldwide: IARC CancerBase No. 11. Lyon, France: 2014. http://globocan.iarc.fr/Pages/fact_sheets_ population.aspx Accessed 4 May 2018.

3. Intergraal Kankercentrum Nederland [Comprehensive Cancer Centre the Netherlands] (IKNL) Cijfers over kanker 2016 [Statistics about cancer] 2016. https://www.cijfersoverkanker.nl/ Accessed 4 May 2018.

4. Arden-Close E, Absolom K, Greenfield DM, Hancock BW, Coleman RE, Eiser C,. Gender differences in selfreported late effects, quality of life and satisfaction with clinic in survivors of lymphoma. Psycho Oncol. 2011;20:1202-1210.

5. Daniels LA, Oerlemans S, Krol AD, van de Poll-Franse LV, Creutzberg CL. Persisting fatigue in Hodgkin lymphoma survivors: a systematic review. Ann Hematol. 2013;92:1023-1032.

6. Kangas M, Bovbjerg DH, Montgomery GH. Cancer-related fatigue: a systematic and meta-analytic review of non-pharmacological therapies for cancer patients. Psychological bulletin. 2008;134(5):700-41.

7. Kreissl S, Mueller H, Goergen H, Mayer A, Brillant C, Behringer K, Halbsguth TV, Hitz F, Soekler M, Shonukan O, Rueffer JU, Flechtner HH, Fuchs M, Diehl V, Engert A, Borchmann P. Cancer-related fatigue in patients with and survivors of Hodgkin's lymphoma: a longitudinal study of the German Hodgkin Study Group. Lancet Oncol. 2016;17:1453-1462.

8. Monterosso L, Taylor K, Platt V, Lobb E, Krishnasamy M, Musiello T, Bulsara C, Stratton K, Joske D. A qualitative study of the post-treatment experiences and support needs of survivors of lymphoma. Eur J Oncol Nurs. 2017;28:62-68.

9. O'Gara G, Tuddenham S, Pattison N. Haemato-oncology patients' perceptions of health-related quality of life after critical illness: A qualitative phenomenological study. Intensive Crit Care Nurs. 2018;44:76-84.

10. Oerlemans S, Mols F, Issa DE, Pruijt JH, Peters WG, Lybeert M, Zijlstra W, Coebergh JW, van de PollFranse LV. A high level of fatigue among long-term survivors of non-Hodgkin's lymphoma: results from the longitudinal population-based PROFILES registry in the south of the Netherlands. Haematologica. 2013;98:479-486.

11. van der Poel MW, Oerlemans S, Schouten HC, Mols F, Pruijt JF, Maas H, van de Poll-Franse LV. Quality of life more impaired in younger than in older diffuse large B cell lymphoma survivors compared to a normative population: a study from the population-based PROFILES registry. Ann Hematol. 2014;93:811819.

12. Allart $P$, Soubeyran $P$, Cousson-Gelie F. Are psychosocial factors associated with quality of life in patients with haematological cancer? A critical review of the literature. Psycho Oncol. 2013;22:241-249.

13. Sant M, Minicozzi P, Mounier M, Anderson LA, Brenner H, Holleczek B, Marcos-Gragera R, Maynadie M, Monnereau A, Osca-Gelis G, Visser O, De Angelis R. Survival for haematological malignancies in Europe between 1997 and 2008 by region and age: results of EUROCARE-5, a population-based study. Lancet Oncol. 2014;15:931-942.

14. Oberoi D, White V, Seymour J, Prince HM, Harrison S, Jefford M, Winship I, Hill D, Bolton D, Kay A, Millar J, Doo NW, Giles G. The course of anxiety, depression and unmet needs in survivors of diffuse large B cell lymphoma and multiple myeloma in the early survivorship period. J Cancer Surviv. 2017;11:329-338.

15. Parker PA, Banerjee SC, Matasar MJ, Bylund CL, Franco K, Li Y, Levin TT, Jacobsen PB, Astrow AB, Leventhal H, Horwitz S, Kissane DW. Protocol for a cluster randomised trial of a communication skills intervention for physicians to facilitate survivorship transition in patients with lymphoma. BMJ Open. 2016;6:e011581.

16. Parry C, Lomax JB, Morningstar EA, Fairclough DL. Identification and correlates of unmet service needs in adult leukemia and lymphoma survivors after treatment. J Oncol Pract. 2012; 8:e135-141.

17. Integraal Kankercentrum Nederland [Comprehensive Cancer Centre the Netherlands ] (IKNL), 2011. Guideline Cancer survivorship care. https://www.oncoline.nl/cancer-survivorship-care Accessed 4 May 2018. 
18. Integraal Kankercentrum Nederland [Comprehensive Cancer Centre the Netherlands] (IKNL), 2017. . Richtlijn Medische Specialistische Revalidatie bij Oncologie. [Guideline Medical Specialist Oncological . Rehabilitation]https://richtlijnendatabase.nl/uploaded/docs/Oncologie/RL_Medisch_Specialistische_Rev alidatie_bij_Oncologie_voor_autorisatie_def.pdf. Accessed 4 May 2018.

19. Newcomb R, Nipp RD, Chan AT, Hochberg EP, Jackson VA, Cashavelly BJ, Wong RL, Greer J, Ryan DP, Temel JS, El-Jawahri A. 2018. Symptom burden in hospitalized patients with curable and incurable cancers. J Clin Oncol. 2018;36 (suppl; abstr 10004).

20. Findley PA, Sambamoorthi U. 2009. Preventive health services and lifestyle practices in cancer survivors: a population health investigation. J Cancer Surviv. 2009;3:43-58.

21. Hall A, Lynagh M, Bryant J, Sanson-Fisher R. Supportive care needs of hematological cancer survivors: a critical review of the literature. Crit Rev Oncol Hematol. 2013;88:102-116.

22. Institute of Medicine. Delivering High-Quality Cancer Care: Charting a New Course for a System in Crisis. Levit LA, Balogh EP, Nass SJ, Ganz PA, editors. Washington, DC: The National Academies Press. 2013:412.

23. Visserman EA, Gijsen BCM, Blaauwbroek HG. Zorgstandaard Kanker [Care Standard Cancer]. Utrecht: Leven met Kanker [Living with Cancer], IKNL, KWF Kankerbestrijding, 2014.

24. Greenhalgh T, Howick J, Maskrey N. Evidence based medicine: a movement in crisis? BMJ. 2014;348:g3725.

25. Parry C, Kent EE, Forsythe LP, et al. Can't see the forest for the care plan: A call to revisit the context of care planning. J Clin Oncol. 2013;31(21): 2651-2653.

26. Lobb EA, Joske D, Butow P, Kristjanson LJ, Cannell P, Cull G, Augustson B. When the safety net of treatment has been removed: patients' unmet needs at the completion of treatment for haematological malignancies. Patient Educ Couns. 2009;77:103-108.

27. Parry C, Morningstar E, Kendall J, Coleman EA. Working without a net: leukemia and lymphoma survivors' perspectives on care delivery at end-of-treatment and beyond. J Psychosoc Oncol. 2011;29: 175-198.

28. Persson L, Hallberg IR. Lived experience of survivors of leukemia or malignant lymphoma. Cancer Nurs. 2004; 27:303-313.

29. Wang CC, Geale SK. The power of story: Narrative inquiry as a methodology in nursing research. Intl J Nurs Sci. 2015;2:195-198.

30. Riessman CK. Narrative Methods for the Human Sciences. Los Angeles, London, New Delhi, Singapore: Sage Publications; 2008.

31. Friesen-Storms JHHM, Bours GJJW, Weijden Tvd, Beurskens AJHM. Shared decision making in chronic care in the context of evidence based practice in nursing. Int J Nurs Stud. 2015;52(1):393-402.

32. Friesen-Storms JH, Bours GJ, Snijders IC, van der Weijden T, Jie KS, Beurskens AJ. A conversation approach based on shared goal-setting and shared decision-making for nurses in cancer aftercare: $A$ developmental study. Eur J Oncol Nurs. 2018;35:107-116.

33. Saunders B, Sim J, Kingstone T, Baker S, Waterfield J, Bartlam B, Burroughs H, Jinks C. Saturation in qualitative research: exploring its conceptualization and operationalization. Quality \& Quantity.2017.

34. Korstjens I, Moser A. Series: Practical guidance to qualitative research. Part 4: Trustworthiness and publishing. Eur J Gen Pract. 2018;24:120-124.

35. Lincoln YS, Guba EG. Naturalistic Inquiry. Newburry Park: Sage; $1985 .$.

36. O'Brien BC, Harris IB, Beckman TJ, Reed DA, Cook DA. Standards for reporting qualitative research: a synthesis of recommendations. Acad Med. 2014;89:1245-1251.

37. World Health Organization (WHO. International Classification of Functioning, Disability and Health (ICF). 2001. http://www.who.int/classifications/icf/en/ Accessed 4 May 2018.

38. Huber M, Knottnerus JA, Green L, Horst H vd, Jadad AR, Kromhout D, Leonard B, Lorig K, Loureiro MI, Meer JW vd, Schnabel P, Smith R, Weel C v, Smid H. How should we define health? BMJ. 2011;343.

39. Sheilds L, Molzahn A, Bruce A, Schick Makaroff K, Stajduhar K, Beuthin R, Shermak S. 2015. Contrasting stories of life-threatening illness: a narrative inquiry. Int J Nurs Stud. 2015;52:207-215.

40. Manne S, Badr H. Intimacy and relationship processes in couples' psychosocial adaptation to cancer. Cancer. 2008;112:2541-2555.

41. Bos-Touwen I. Tailoring Self-Management in Chronic Care. Utrecht: Utrecht University; 2016. 
42. Howell D, Harth T, Brown J, Bennett C, Boyko S. Self-management education interventions for patients with cancer: a systematic review. Support Care Cancer. 2017;25:1323-1355.

43. Howell DD. Supported self-management for cancer survivors to address long-term biopsychosocial consequences of cancer and treatment to optimize living well. Curr Opin Support Palliat Care. 2018.

44. Kim SH, Kim K, Mayer DK. Self-Management Intervention for Adult Cancer Survivors After Treatment: A Systematic Review and Meta-Analysis. Oncol Nurs Forum. 2017;44:719-728.

45. Coulter A, Entwistle VA, Eccles A, Ryan S, Shepperd S, Perera R. Personalised care planning for adults with chronic or long-term health conditions. Cochrane Database Syst Rev. 2015;Cd010523.

46. Banerjee SC, Matasar MJ, Bylund CL, Horwitz S, McLarney K, Levin T, Jacobsen PB, Parker P, Astrow A, Kissane DW. Survivorship care planning after participation in communication skills training intervention for a consultation about lymphoma survivorship. Transl Behav Med. 2015;5:393-400.

47. Franco K, Shuk E, Philip E, Blanch-Hartigan D, Parker PA, Matasar M, Horwitz S, Kissane D, Banerjee SC, Bylund $\mathrm{CL}$. Communication between oncologists and lymphoma survivors during follow-up consultations: A qualitative analysis. J Psychosoc Oncol. 2017;35:513-530.

48. Hudson SV, Ohman-Strickland PA, Bator A, O'Malley D, Gundersen D, Lee HS, Crabtree BF, Miller SM. Breast and prostate cancer survivors' experiences of patient-centered cancer follow-up care from primary care physicians and oncologists. J Cancer Surviv. 2016;10:906-914.

49. Klaassen L, Dirksen C, Boersma L, Hoving C. Developing an aftercare decision aid; assessing health professionals' and patients' preferences. Eur J Cancer Care. 2018;27(2): e12730.

50. Benning TM, Kimman ML, Dirksen CD, Boersma LJ, Dellaert BG. Combining individual-level discrete choice experiment estimates and costs to inform health care management decisions about customized care: the case of follow-up strategies after breast cancer treatment. Value Health. 2012;15:680-689.

51. Kimman ML, Dellaert BG, Boersma LJ, Lambin P, Dirksen CD. Follow-up after treatment for breast cancer: one strategy fits all? An investigation of patient preferences using a discrete choice experiment. Acta Oncol. 2010;49:328-337.

52. Stacey D, Legare F, Lewis K, Barry MJ, Bennett CL, Eden KB, Holmes-Rovner M, Llewellyn-Thomas $H$, Lyddiatt A, Thomson R, Trevena L. Decision aids for people facing health treatment or screening decisions. Cochrane Database Syst Rev. 2017;4, Cd001431. 


\section{Chapter

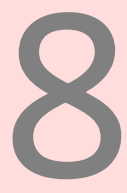

General discussion

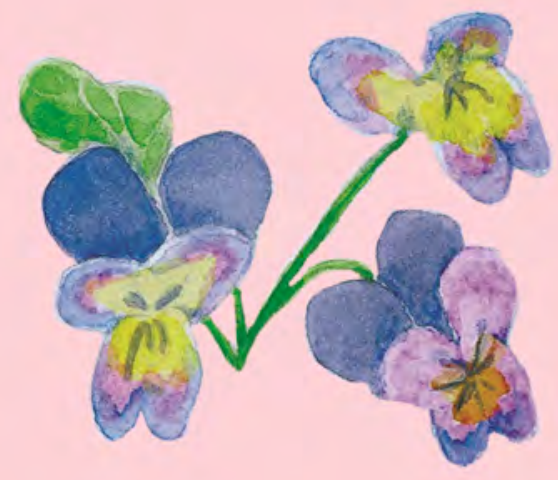





\section{General discussion}

This chapter presents an overview of the main findings of the studies belonging to this research project. It also addresses general methodological considerations, theoretical considerations and comparison with current literature. Finally, the chapter provides conclusions and implications of the research for nursing practice, education and research.

The aim of the research project was to gain knowledge about the implementation of evidence-based practice (EBP) in nursing, to find a way to integrate shared decision making (SDM) with EBP in a chronic care environment in nursing, and to develop a strategy for an integrated approach of EBP and SDM in daily nursing practice in the individual aftercare for cancer survivors.

We applied various research methods to answer the following main research questions:

1. What is the feasibility of implementing EBP in daily nursing practice by interactive and clinically integrated teaching methods?

2. How can shared decision making be integrated with evidence-based practice in chronic nursing care?

3. Which strategy, tailored to the needs of patients and proxies, nurses and other health care professionals, seems worthwhile and feasible to implement an integrated approach of evidence-based practice and shared decision making in cancer aftercare?

\section{Main findings}

This section addresses the main findings of this research project. The main findings of each research question were used as input for the next research questions.

\section{Feasibility of implementing evidence-based practice in daily nursing practice}

In order to gain optimal insight into the implementation of EBP we conducted two studies (described in Chapter 2 and 3 ) in which EBP was being implemented in nursing. In the first study we used a participatory action research design and in the second study we used a systematic implementation model with elements of action research. In both studies we used interactive and clinically integrated teaching methods. 
The first study (described in Chapter 2) led to the development and implementation of an evidence-based discharge protocol. The second study (described in Chapter 3) led to several 'patient case discussion meetings'. Nurses searched and discussed research literature for information about how to measure pain, which subsequently led to an implementation proposal for a pain protocol. Both studies showed that, although a systematic approach was used to implement EBP and the interventions were based on barriers and facilitators, implementation of EBP was still difficult in the nursing settings. Although nurses were able to search for English literature and guidelines, all nurses, even those with a bachelor's degree, found it difficult to search for and appraise literature in a systematic way. To improve the feasibility of implementing EBP in nursing, we learned that it is important to simplify the EBP steps to suit the level of education of the nurses, for example focusing on major care topics and on pre-appraised evidence such as (Dutch) clinical practice guidelines, critically appraised topics or systematic reviews. In addition, we found that for a sustainable implementation of EBP organisational changes are needed that foster a culture and knowledge of EBP, like appointing nurse practitioners or other nurses with a master's degree to help the nurses on the ward with the EBP process steps.

\section{The integration of shared decision making with evidence-based practice in chronic nursing care}

In a discussion paper (Chapter 4) we discussed the relevance of SDM making in chronic care care as well as how SDM can be integrated with EBP in nursing.

Our implementation studies and other research related to EBP, merely emphasise the use of research evidence in clinical practice, with little focus on the individual patient's values and clinical expertise in the decision-making process. We acknowledge the importance of integrating SDM with EBP because SDM includes the individual patient's values into the decision-making process. In nursing, SDM seems particularly relevant in chronic care because it may enhance self-management, self-efficacy, and patient empowerment. In chronic care, self-management is inescapable because patients are faced with decisions that influence the course of their disease on a daily basis.

We integrated SDM with the decision-making step of EBP in chronic care. Although we believe that in chronic care it is important to always invite the patient to participate in the decision-making process to include the individual patient's values, in our opinion, the degree of SDM depends on different attributes of health care interventions: the level of research evidence, the presence of intervention options, the burden of side effects, the impact on life style, patient group values and the impact on resources. Patient values become even more important in cases where the level of research evidence is low, there 
are multiple intervention options, the burden of side effects of the interventions options is high, the impact of the intervention options on life style is high, patient group values are heterogeneous, and there is a high impact on resources.

Next to the attributes of health care interventions the patient's willingness to participate in SDM, the clinical expertise, knowledge, skills and attitude of the nurse towards SDM, and the context in which the decision making takes place affect the affect the SDM process. Providing nurses with proper training, clinical guidelines and decision support tools for patients linked to these guidelines are essential to support implementation of SDM by nurses.

\section{Development and feasibility of an integrated approach of evidence-based practice and shared decision making in cancer aftercare}

The knowledge gained from answering research questions 1 and 2 is used to answer the third research question by developing and using EBP and SDM as an integrated approach (See Figure 8.1) in a conversation approach for a cancer aftercare consultation.

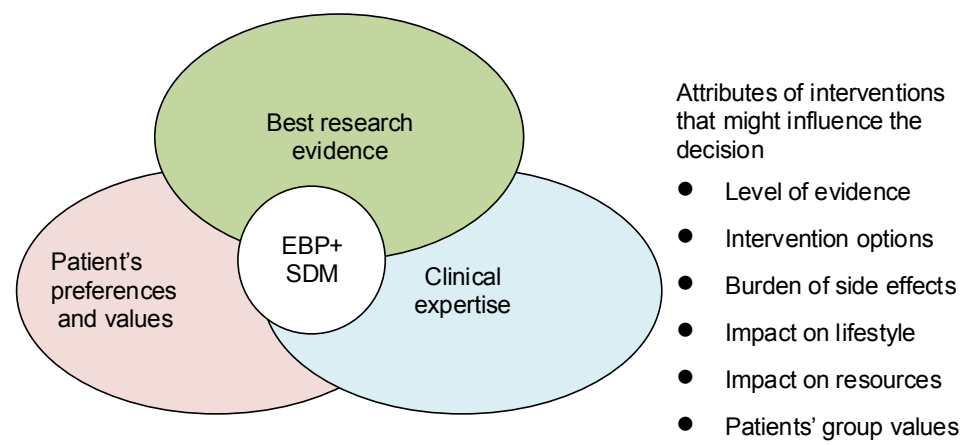

Figure 8.1 An integrated approach of EBP and SDM.

To develop this conversation approach to improve cancer aftercare, we cooperated with a large teaching hospital in the South of the Netherlands and used an iterative usercentred development process for complex interventions in nursing (described in Chapter 5). Next, we studied, facilitators of and barriers to an aftercare decision aid (PtDA) for patients with breast cancer developed by Klaassen et al. ${ }^{1-2}$ in which we had integrated tools based on our conversation approach (described in Chapter 6). Lastly, we studied the experiences of lymphoma survivors in early aftercare after having been exposed to the conversation approach (described in Chapter 7). 
The conversation approach for cancer aftercare consists of four phases: 1 . Preparation of the consultation including patient reported outcome measures (PROMs) (The Distress Thermometer with the Problem List $^{3}$ and the Screening Inventory Psychosocial Problems ${ }^{4}$ ), 2.Shared goal setting by means of a tool visualizing domains of life $e^{5}, 3$. Shared care planning by means of an overview of possible choices in aftercare, a database with health care professionals and a cancer survivorship care plan, and 4 . Evaluation. The conversation approach leads to an individualised care plan.

Our conversation approach was based on a conversation approach for shared goalsetting and decision making for chronic patients in primary care ${ }^{6-7}$, in which the prevailing SDM model ${ }^{8-9}$ was expanded with goal setting. ${ }^{10}$

Although the development and use of a conversation approach appears to be worthwhile, in nursing a standard nursing process exists. Despite the fact that nurses often have been educated to work according to this nursing process, it seems not well implemented in daily practice. ${ }^{11}$

The developed conversation approach and the standard nursing process consist of comparable processes. However, the conversation approach more explicitly includes SDM integrating patient goals and values with clinical expertise and EBP, and therefore enriches the standard nursing process. An overview of the way the conversation approach and the systematic nursing approach compare to each other, is presented in Figure 8.2.

A pretest of the conversation approach with nurses $(n=4)$ and patients $(n=11)$ revealed that the approach needs to be flexible, tailored to the patient and practice setting, and embedded in care processes. During the pretest, the conversation approach was perceived as enhancing patient-centredness and leading to more in-depth consultations. However, nurses experienced it as a substantial challenge to elicit patients' preferences and set goals together with patients. Another challenge experienced during the pre-test, was the time-consuming nature of the approach. The nurses suggested to send a questionnaire home to the patient before the consultation or to digitise the questionnaire and send a link to the patient.

Subsequently, we digitised and integrated some tools (including the questionnaire) based on our conversation approach in a PtDA for aftercare in breast cancer ${ }^{1-2}$, as suggested during the pretest of the conversation approach (See Appendix 8.1 for print screens of the PtDA) (Link to PtDA: https://beslissamen.nl/pda_launch.html?pda=tools/ pda_borstnazorg_nl/story_html5.html).

An adoption study (described in Chapter 6 ) was conducted to assess facilitators of and barriers to implementation of the PtDA for aftercare in breast cancer to tailor the implementation strategy to. When looking at determinants associated with the PtDA, the determinants compatibility and relevance had a significant positive association with 
the intention of health care professionals to adopt the PtDA. When looking at determinants associated with the adopter, the determinants self-efficacy and outcome expectations were significantly related with the intention to adopt the PtDA. Finally, when looking at determinants associated with the organisation and the socio-political context only adherence to the guideline had a significant association with the intention to adopt. When all determinants were analysed together only self-efficacy and relevance for the patient remained significantly associated with the intention of health care professionals to adopt the PtDA.
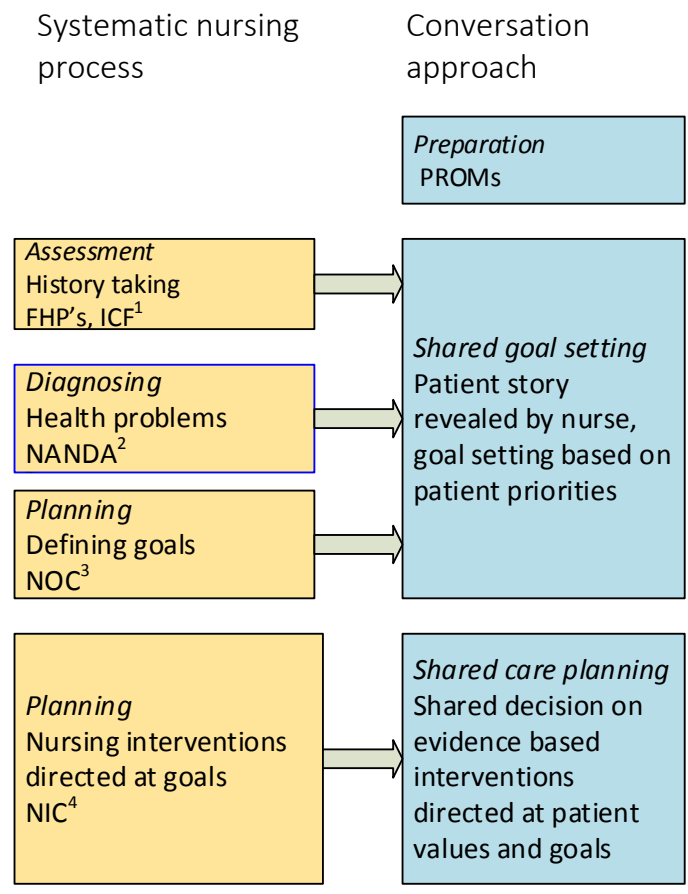

\section{Implementation \\ Carrying out care plan}

\begin{tabular}{|l|l|}
\hline $\begin{array}{l}\text { Evaluation } \\
\text { Evaluating and } \\
\text { modifying care plan }\end{array}$ & \begin{tabular}{|l|} 
Evaluation \\
Evaluating and \\
modifying care plan
\end{tabular} \\
\hline
\end{tabular}

Figure 8.2 The conversation approach compared to the systematic nursing process

${ }^{1}$ Functional Health Patterns (FHP) proposed by Marjorie Gordon ${ }^{12}$ for history taking. International classification system of functioning, disability and health. ${ }^{13}$

2 The North American Nursing Diagnosis Association (NANDA) provides a classification system of nursing diagnoses. ${ }^{14}$

${ }^{3}$ Nursing Outcomes Classification (NOC) $)^{15}$ and Nursing Intervention Classification (NIC) ${ }^{16}$, both classification systems for standardized nursing outcomes and interventions. 
In the final study (described in Chapter 7) lymphoma survivors were exposed to the conversation approach (which was based on an integration of EBP and SDM) in the setting of an aftercare consultation. We found that participants had to deal with health consequences like fatigue and lengthy absence from work. Their experiences and strategies for dealing with the consequences of the lymphoma varied. The respondents reacted differently in coping with the disease and its related uncertainty. The social support they received from relatives and friends was greatly valued, although some social relationships had deteriorated. Respondents not only experienced tough times from their treatment. Life events relating to the cancer treatment, as well as their lives in general, caused tough times to become part of their life stories. There were also differences in the extent to which respondents experienced an added value of the aftercare solution as well as felt the need for further consultations. The aftercare consultation frequently resulted in initiatives for interventions, like oncological rehabilitation or oncological physical therapy, social work or psychological therapy.

\section{Methodological considerations}

This section reflects on some overarching methodological considerations in the research project, in addition to discussing methodological issues that were already brought up earlier on in the thesis. These overarching methodological considerations are related to: building bridges across the paradigm shift of evidence-based practice, action research, trustworthiness of qualitative studies, and implementation in a dynamic context.

\section{Building bridges across the paradigm shift of evidence-based practice}

While this research project was being undertaken $a(n)$ (inter)national paradigm shift occurred with regard to EBP. More specifically, the singular focus on research evidence in EBP gave way to a more multifaceted focus, in which not only research evidence, but also the values of the individual patient and the patient's context were taken into account. ${ }^{17-19}$ To actually practically take the values and contexts of individual patients into account the integration of EBP and SDM seemed necessary. ${ }^{20-21}$ In a recent Dutch national report the term context-based practice was suggested to replace the term evidence-based practice. ${ }^{17}$ This societal paradigm shift is echoed in this thesis, as we tried to build bridges between the classical EBP interpretation (Chapter 2 and 3 ) and the proposed context-based practice. To do so, we used a variety of study designs, suggesting how to integrate EBP and SDM in chronic care, especially in cancer aftercare, to include the values of the patient in the decision-making process. Through building 
bridges and thereby focusing on several topics within this thesis, the cohesion of the studies in the different parts of this thesis might not be obvious to every reader. We, however, regard this illustration of bridging paradigms, as a strength of this thesis.

\section{Action research}

During the research project, we used elements of action research in the two implementation studies (Chapter 2 and 3) and for the development of the conversation approach (Chapter 5). Action research is an umbrella term that includes several traditions of theories and research practice that aim to develop a research process that addresses issues of concern. Action research brings together research and action, theory and practice, academic and local knowledge and participation of people in research. ${ }^{22-25}$ The strength of action research is that it acknowledges the nature of a research study as a complex social process that yields knowledge, based on studies conducted within practical contexts. ${ }^{24}$ Furthermore, if an action research approach is systematic and iterative and involves the target group of an innovation (that is tailored to a practice setting) in the development and implementation of that innovation, it might promote implementation. $^{26-27}$

A strength of the research project is that we chose action research designs that not only fit the aim but also the setting of the studies. In the first study we used a participatory action research design, in the second study, we chose a systematic implementation model with elements of action research and in the fourth (developmental) study, we used an iterative user-centred developmental process for complex interventions in nursing that was derived from the well-known Medical Research Council (MRC) framework. ${ }^{28-29}$ This developmental process highlighted a number of important activities required for intervention delivery, for example the need to identify the goal of the intervention, the need to build the intervention and the need to take cognizance of the scope of nursing practice, all of which are implicit in the MRC framework guidelines.

A further strength of our research project is the involvement of stakeholders. In the developmental study, we explicitly invited patients as equal partners ${ }^{30}$ in an expert meeting in the process of setting up the conversation approach for cancer aftercare. Involving patients a phase in the process of setting up the conversation approach is part of an increasing attention on Public and Patient Involvement (PPI), defined as 'research being carried out "with" or "by" members of the public rather than "to", "about" or "for" them'. 


\section{Trustworthiness of qualitative studies}

In this research project we mainly conducted relatively small qualitative studies within a specific context. This may limit the extent to which the findings of the current research project can be extrapolated to wider populations. ${ }^{33}$ An additional limitation of this research project specifically concerns the implementation studies that were conducted. For practical reasons, these studies were both conducted among staff from pulmonary wards. It might have led to additional insights if we would have been able to include staff from a variety of wards.

We enhanced transferability of the results of our qualitative studies to other contexts by providing the readers with a 'thick description' of the study setting, the participants and the research process of the different studies. ${ }^{34}$ Subsequently, the reader may decide which results fit best to their own setting and are worthwhile transferring and translating. We tried to enhance the trustworthiness of the qualitative studies in terms of credibility, dependability, and confirmability in several ways. ${ }^{34-36}$ Throughout all studies extensive peer debriefing took place with both members of the research team (for credibility purposes) and other (expert) researchers (for dependability purposes), about the study design, data collection, analysis, and the interpretation of findings. Investigator triangulation was used by involving several researchers in the data collection and/or analysis of the data, as well as involving them in addressing the organisational aspects of the studies (for credibility purposes). Researchers in all study settings were involved/engaged for prolonged periods of time, and this resulted in them being familiar with the study setting (which aids credibility of the studies). We also conducted informant triangulation by involving multiple stakeholders in our studies (for credibility purposes). During the iterative design of several studies the data was continuously tested by stakeholders (for credibility and dependability purposes). Furthermore, the researcher continuously reflected on the studies conducted within this research project, on interactions with and between people within settings, and on interviews and analyses with other researchers and in reflexive notes (for confirmability purposes).

\section{Implementation in a dynamic context}

As is the case in many studies, unforeseen circumstances made it necessary to adjust the implementation of the intended set-up and methodology of this research project At the start of our research project in cancer aftercare we had planned to conduct a larger process evaluation ${ }^{28-29}$ with quantitative and qualitative measures in the malignant lymphoma and breast cancer care pathway. A quantitative measure was the degree to which SDM took place as assessed with both the CollaboRATE ${ }^{37}$ and the 9-item Shared 
Decision Making Questionnaire (SDMQ-9) ${ }^{38}$ in a pre-posttest design. However, during the pretest period of the quantitative measure, the hospital that cooperated in our study merged with another hospital in the South of the Netherlands. Due to barriers that will be discussed in the theoretical considerations, it was not possible to implement the conversation approach in the aftercare of breast cancer patients. As a result, we were not able to conduct the process evaluation, which in turn, meant it was not possible to study implementation of our conversation approach, let alone to study its process and effects.

This unforeseen circumstance, however, did bring about a positive result as well, in the form of an opportunity to cooperate with Prof. Dr. Liesbeth Boersma and Linda Klaassen MSc that developed a PtDA for aftercare in breast cancer ${ }^{1-2}$ to improve this PtDA and digitise and integrate tools based on our conversation approach in the PtDA. Subsequently, an adoption study concerning this PtDA was conducted. Another opportunity that occurred as a result of not being able to conduct the process evaluation, was that it enabled us to conduct a narrative inquiry among lymphoma survivors to reach in-depth insight in their experiences in early aftercare. The evidence acquired from the narrative inquiry is considered to be important in informing and enabling health care professionals so that they can deliver patient-centred evidencebased aftercare in a better way. ${ }^{39}$ However, the limitation of not being able to conduct a larger process evaluation and the resulting choice to move forward in the field of breast cancer and malignant lymphoma aftercare with different studies, has led to less cohesion between the studies concerning cancer aftercare, in part three of this thesis.

\section{Theoretical considerations and comparison with current literature}

This research project provides new insights in how to build bridges across the paradigm shift of EBP, how evidence-based aftercare in cancer can be personalised by using a conversation approach that integrates and EBP and SDM, and in barriers experienced during implementation in a dynamic context. In this section our results are put into the context of the existing literature.

\section{Building bridges across the paradigm shift of evidence-based practice}

As already mentioned in the Methodological Considerations section in this chapter, we tried to build bridges across the paradigm shift that occurred with regard to EBP. The singular focus on research evidence in EBP gave way to a more multifaceted focus, in 
which not only research evidence, but also the values of the individual patient and the patient's context were taken into account. ${ }^{17-19}$ The paradigm shift was visible in two perspectives.

First, there was a shift in the perspective of which sources of research evidence are used. While EBP scholars used to focus on the search, critical appraisal and use of the research results from a single primary epidemiological study, they increasingly adopted the view that it is important to integrate summarised, critically appraised and translated best evidence from several well-defined studies on clinical practice. ${ }^{40-43}$

Internal factors (individual readiness) and external factors (organisational readiness) complicate the implementation of EBP in nursing. ${ }^{40,44-45}$ Moreover, the process steps of EBP are difficult for nurses, especially the critical appraisal of studies. ${ }^{40,44-46}$ The lessons learned from our implementation studies ${ }^{44-45}$ were in line with the growing opinion of EBP scholars that the focus in the integration of different kind of sources of research evidence should lie on the integration of summarised, critically appraised and translated best evidence, such as recommendations from high quality ${ }^{47-48}$ evidence-based clinical practice guidelines, into clinical practice.

The use of different kinds of sources of research evidence may vary according to the educational level of nurses. More specifically speaking: secondary vocationally or medium level trained nurses have to be able to follow clinical practice guidelines that have been translated into protocols (making them EBP followers). Bachelor nurses are trained to be 'EBP users', and have to be able to formulate a PICO question, search summarised literature and evaluate the quality and practical usability of the evidence and assist EBP followers. Master nurses are trained to conduct all process steps of EBP (making them EBP experts) and carry out complex literature searches and critical appraisal of single research studies to develop critically appraised topics. Furthermore, they assist the bachelor and secondary vocationally trained nurses (EBP followers and users) with EBP. ${ }^{49-51}$

An example of an initiative that was recently launched in the Netherlands, that may support nurses to implement evidence-based nursing care from clinical practice guidelines, assessed low-value care practices in Dutch clinical practice guidelines. ${ }^{52}$ This low value care is defined 'do-not-do' recommendations in clinical practice guidelines. The aim of this initiative was to stimulate and support nurses to reduce the prevalence of this type of care. ${ }^{52}$

Next to the lessons we learned about the difficulty of conducting the process steps of $E B P$, we also learned from our implementation studies that it is difficult for nurses to prioritise which nursing care would be most worthwhile and feasible to improve by implementing EBP. ${ }^{44-45}$ Our advice to focus implementation of EBP around major care 
topics like pain and nutrition ${ }^{44-45}$, is in line with other studies in which the focus lies on pain management interventions ${ }^{53}$ or on major basic care themes (bathing and dressing, communication, mobility and nutrition). ${ }^{54}$

Second, aside from the shift that occurred in the perspective of which sources of research evidence are used, a shift occurred in the perspective of patient values in EBP. In the most common definition of EBP, patient values are clearly described as being an important part of the decision-making process. ${ }^{55}$ However, education and research about EBP has so far mainly focussed on the use of research evidence in clinical practice 56-57, leaving patient values untouched. As a result, there was a growing opinion that the patient's values and the patient's context should receive more attention in EBP. ${ }^{17-19,41,57-58}$ SDM seemed necessary to incorporate patients' values in the decisionmaking process of EBP. ${ }^{20-21,59-66}$

Since the early 1990s, SDM had gained considerable interest in option-sensitive or preference-sensitive decisions regarding medical diagnostic or treatment options. Decision aids $^{67}$ directed at medical screening and treatment decision making were developed to facilitate the process of SDM. Some authors acknowledged that SDM was also relevant in nursing in chronic care. ${ }^{59-60}$ In this thesis, we advocate that in chronic care it is important to always invite the patient to participate in the decision-making process. We furthermore propose attributes from the GRADE system ${ }^{68}$ that can be incorporated in a SDM conversation approach in chronic care. These attributes can be used like the personalised risk communication for medical decisions ${ }^{69-70}$ to carefully consider the different intervention options with the patient, while, for example, taking into account the impact these different options might have on lifestyle and resources. In addition, we facilitated the use of SDM in cancer aftercare by digitising and integrating tools of our conversation approach in an aftercare decision aid for breast cancer patients. $^{1-2}$

Insights in both perspectives of the paradigm shift are useful for clinical nurse leaders who might facilitate implementation of EBP and SDM within an organisation. ${ }^{71-75}$ The new education profile of the bachelor of nursing $2020^{76}$, indicates that bachelor nurses are educated to become clinical nurse leaders. The educational profile describes the competencies of these nurses according to the 'Canadian Medical Education Directives for Specialists' (CAN meds) roles. The CAN meds role 'reflective EBP professional' outlines the EBP competencies for future nurses and in the CAN meds role 'Collaborator', SDM competencies are outlined. 


\section{Personalised evidence-based aftercare in cancer}

Our studies in the field of cancer aftercare ${ }^{77-79}$ contribute to the literature in that field, because we offer a conversation approach that supports SDM in evidence-based cancer aftercare. In addition, the narrative inquiry provided in-depth insight in experiences of lymphoma survivors in early aftercare.

The studies addressed several important topics that take up a prominent place in the literature, such as using patient-reported outcome measures (PROMs) to guide clinical decision making, using shared goal setting and SDM in cancer aftercare, and the support of patients in the transition from the patient phase to the survivorship phase. In this section these topics are discussed.

\section{Using patient-reported outcome measures}

We integrated existing PROMs in or conversation approach to detect issues that patients experienced after cancer treatment, in order to facilitate a discussion of the needs and goals of patients in the decision-making process conducted in aftercare. A PROM is defined as 'any report coming directly from the patient about a health condition and its treatment' using a self-reported measure. ${ }^{80}$ PROMs are advocated for use in routine cancer clinical practice. ${ }^{80-82}$ The use of PROMs may be supportive in clinical decision making. ${ }^{80,83-85}$ It may support a better detection of symptoms ${ }^{86}$, facilitate the identification of need for patient referral ${ }^{80,87}$, and more actions taken by clinicians to address patients' needs. ${ }^{80,88}$ In daily practice, however, PROMs are often not integrated well in routine care to actually use the results of the PROMs immediately to guide actions of health care professionals. ${ }^{80,89-90}$ In our studies, the integration of PROMs in a visual tool, called 'the Four Domains of Life', that was set up as part of the conversation approach, enabled the immediate discussion of the results of the PROMs during consultations. Other initiatives exist that may support consultations, in similar ways, like the 'spider web model' from the concept of 'Positive Health' ${ }^{91}$

\section{Shared goal setting and shared decision making}

The conversation approach that we propose, aims at shared goal setting and shared decision making in daily practice for cancer aftercare. Both of these practices are considered important in cancer care. ${ }^{92-95}$ The Dutch Guideline on aftercare ${ }^{96}$ and the Dutch Care Standard Cancer ${ }^{97}$ recommend that goal setting and SDM are practised in aftercare. The 2017 ASCO communication guideline ${ }^{98}$ recommends that clinicians establish care goals with patients. Care goals and the treatment decisions that are made 
based on these goals should be aligned with patient values and priorities as much as possible.

Goal setting was not an explicit element of the integrative definition of shared decisionmaking put forth by Makoul et al. ${ }^{99}$ In early decision making models ${ }^{8}$, no goal setting phase was integrated. The models focused only on medical and pre-defined rather fixed treatment decisions for example choosing between breast conserving surgery and a mastectomy. In our discussion paper $^{56}$ we discussed using the in medicine originated 'three- talk model' of Elwyn et al. ${ }^{8}$ for decisions in chronic care. We, however, did not yet integrate an explicit goal-setting phase in the model of Elwyn et al. ${ }^{8}$

As we moved further with our research project in cancer aftercare, the need for supporting patients' self-management ${ }^{91,100-102}$ and the potential of goal setting to aid in the support of self-management, increasingly became a part of SDM in chronic care practice. 6,93-94,102-104 However, in literature, the mutual relation of 'shared decisionmaking' and 'goal setting' is not yet clearly defined. ${ }^{103}$ Some authors focused on shared decision-making within goal setting. ${ }^{105}$ In medical decision making, goal setting received attention in an updated model of the 'three-talk model' of Elwyn et al.. ${ }^{8}$ The 'choice talk' of this model was replaced by a 'team talk', that incorporates the elicitation of patient goals. ${ }^{106}$ However, aside from elicitation of patient goals in a 'team talk' a 'goal talk' could also be viewed as a separate phase of a shared decision-making process. ${ }^{7,104,107}$

We cooperated with Lenzen et al. ${ }^{6}$ and opted for adding 'a goal talk, as a separate phase to the framework of the conversation approach. We think, this fits best with the setting in which the conversation approach is used during an aftercare consultation as a distinct moment in time to help people transit from the treatment phase to the survivorship phase, and to personalise care based on the goals, values and preferences of the patient. $^{78}$ After setting goals, during the aftercare consultation, the patient and the nurse together decide about possible aftercare options which might support patients to self-manage their goals or receive support from other health care professionals to manage their goals. ${ }^{78}$

A similar framework was developed for SDM with frail elderly persons. ${ }^{104}$

\section{Transition from the patient to the survivorship phase}

Our studies on cancer aftercare focus on conducting a structured aftercare consultation after completion of active treatment. ${ }^{96-97}$ EBP guidelines and other literature ${ }^{108-110}$ recommend aftercare consultations to assist patients in the transition to the survivorship phase. However, the initiation of aftercare or survivorship care, as it is often called in literature, can already start at the moment of cancer diagnosis, instead of at the moment of completion of active treatment. ${ }^{110}$ Starting this process early allows the 
health care professional and patient to plan for the (post-)treatment period, laying the groundwork for a better continuity of care for the patient. ${ }^{111}$ For example, there is evidence that being physically active from pre-diagnosis to post-treatment in breast cancer is beneficial for psychological wellbeing of survivors. ${ }^{112}$ Patients in our narrative inquiry $^{78}$ reported that they were provided with information about evidence-based aftercare during the treatment phase. Patients being provided with information about evidence-based aftercare during the treatment phase, is, however, not structurally embedded within the developed aftercare approach and integrated within work processes.

In the broader context of health care there is an ongoing discussion about how and where aftercare for patients treated for cancer should be organised. Within the Dutch Health Care System the care of people suffering from chronic diseases has been shifting from secondary to primary care. ${ }^{113}$ Primary care physicians and nurses are positive about mobbing cancer aftercare from hospital care to primary care. Part of the aftercare is already conducted in primary care, but it is often not structurally organised. ${ }^{114}$ However, to shift aftercare from hospital to primary care in a structural and organised manner, inter-professional collaboration as well as the knowledge primary care providers have about cancer has to be optimised. ${ }^{114-115}$ Aftercare models that are shared between oncological hospital care and primary care might be valuable. ${ }^{96,116-117}$

\section{Implementation in a dynamic context}

As already stated in the methodological considerations, it was not possible to implement the conversation approach in the aftercare of breast cancer patients in the dynamic context of the merge of two hospitals.

Multiple frameworks ${ }^{118-119}$ reflect on factors influencing implementation of health interventions. In this section we focus on the main implementation barriers and we compare the main barriers to implementation that we came across in this study to barriers described in the existing literature.

The first implementation barrier was that we could not create a sense of urgency about the necessity to implement the conversation approach among the nurses of the new, merged hospital. In addition, the nurse practitioner of the breast cancer pathway of the first hospital that we engaged in the development of our conversation approach, changed jobs. This nurse had a positive attitude towards the conversation approach and was a driving force behind the implementation of the approach. The nurses of the breast cancer pathway in the second hospital were not involved in the developmental process, and these nurses did not feel a sense of urgency to implement the conversation 
approach. Literature indicates that in complex interventions, where a change of behaviour of health care professionals is important, strong stakeholder involvement and user engagement as well as being able to create a sense of urgency is necessary. ${ }^{26,120}$ This was also highlighted in our adoption study of the decision aid. ${ }^{79}$

The second implementation barrier was an ongoing major change in the organisation of the breast cancer care pathway after the merge of the hospitals. The context of the merged hospital differed from the context of the primary hospital in which we conducted our study, which complicated transferability of the conversation approach. ${ }^{121}$ In addition, nurses and nurse practitioners were pressed for time during the merge and they felt too overburdened to participate in a study of the conversation approach. Such a lack of time, as well as experiencing difficulties in integrating interventions in organisational processes are often brought up in literature as important barriers to (successful) implementation. ${ }^{26,77,79}$

\section{Conclusions}

In the common definition of EBP, patient values are integrated with research evidence and clinical expertise in the decision-making process for an individual patient. The use of research evidence seems to receive major attention in the education of EBP, while patient values are neglected. Conducting the process steps of EBP is difficult for nurses, especially for secondary vocationally trained nurses. We advise, as does other literature, to integrate summarised, critically appraised, and translated research, like evidencebased clinical practice guidelines, into evidence-based decision making. In addition, during the research project, the opinion that more focus should be put on the patient's values and the patient's context was growing on an (inter)national level. We suggest to always invite chronic patients in the decision-making process and propose how to integrate EBP and SDM. We developed a conversation approach (in which both EBP and SDM are integrated) for use in cancer aftercare, which incorporates PROMs and visual tools, and supports goal setting. Using the conversation approach might lead to more indepth conversations and as such lead to support for patients in receiving personalised aftercare, specifically with relation to a variety of problems they experience in early aftercare. The approach needs to be embedded within care processes and nurses should be trained to use the approach. Implementation of health care interventions, such as the developed conversation approach, in the dynamic context of a merge is challenging. 


\section{Implications}

In this last section the implications of our research project for nursing practice, education and research are discussed.

\section{Nursing practice}

For nurses, especially secondary vocationally or medium level trained nurses, it is difficult to conduct the process steps of EBP. We suggest adapting the EBP process steps to suit the level of education of the nurses. This can be done by formulating a PI question or PICO questions around major care topics like pain and nutrition, and advising a search strategy focussing on (Dutch) clinical practice guidelines and other summarised evidence, such as systematic reviews and critically appraised topics. To teach and implement EBP, it seems important to link EBP with to the context in which it is implemented for example by choosing authentic patient cases.

In addition, we advise the hospital management to appoint nurse practitioners or other nurses with a master's degree to help the nurses on the ward with the EBP process steps, especially regarding efficiently searching the research literature and critically appraising the summarised evidence or guidelines.

It is important to always invite the chronic care patient to participate in the decisionmaking process. For consultations in chronic care, we suggest using a systematic conversation approach that integrates EBP and SDM, incorporates PROMs and visual tools, and supports a separate 'goal talk'. The level of the quality of research evidence, the side effects of intervention options, the impact of intervention options on the patient's life style, the patient's values, and the impact of intervention options on resources can be discussed to help patients in weighing the different intervention options. In addition, we suggest using the conversation approach to guide the implementation of EBP and SDM in daily practice and dialogue conversations.

It is important that patients receive personalised aftercare. We suggest that oncology nurses conduct an aftercare consultation with cancer survivors within the hospital and subsequently sharing further aftercare tailored to the wishes of the individual cancer survivor between hospital and primary care. In order to do this, it is important that networks, knowledge and inter-professional collaboration are optimised.

\section{Education}

We recommend that nurse educators integrate EBP and SDM and use a conversation approach, like the one we developed, in the curriculum. We, further, recommend that 
secondary vocationally or medium level trained nurses are taught the competencies to use EBP guidelines and protocols and to support self-management of patients. In the education of bachelor nurses we advise to follow the new education profile of the bachelor of nursing $2020 .^{76}$ We propose that nurses are taught the EBP and SDM competencies and that education starts with critical clinical reasoning competencies. Subsequently, to teach nursing students the competencies to conduct the process steps of EBP focused on major care topics and to integrate (Dutch) evidence-based clinical guidelines in the SDM process for authentic patient cases. Subsequently, students can be taught to search summarised, critically appraised evidence, like systematic reviews and other research studies in databases like PubMed. To understand the level of the quality of research evidence they need knowledge about different research designs.

\section{Research}

Due to barriers that will be discussed in the theoretical considerations, it was not possible to implement the conversation approach in the aftercare of breast cancer patients. As a result, we were not able to conduct the process evaluation, which in turn, meant it was not possible to study implementation of our conversation approach, let alone to study its process and effects. We advise to further study factors that influence effective implementation of the conversation approach in a process evaluation according to the MRC framework ${ }^{28}$ and to study the effect of the conversation approach in a broader setting. In addition, we advise to carefully reconsider conducting research on or implementing complex interventions in a dynamic context, such as might exist during a merge.

It is important to start with the initiation of survivorship care early on in the cancer care trajectory. We suggest exploring how the underlying concept and tools of the conversation approach can be used earlier on in the care trajectory. 


\section{References}

1. Klaassen L, Dirksen C, Boersma L, Hoving C. Developing an aftercare decision aid; assessing health professionals' and patients' preferences. Eur J Cancer Care. 2017; e12730.

2. Klaassen LA, Dirksen CD, Boersma LJ, Hoving C, Portz MJG, Mertens PMG, et al. A novel patient decision aid for aftercare in breast cancer patients: A promising tool to reduce costs by individualizing aftercare. The Breast. 2018;41:144-50.

3. Tuinman MA, Gazendam-Donofrio SM, Hoekstra-Weebers JE. Screening and referral for psychosocial distress in oncologic practice: use of the Distress Thermometer. Cancer. 2008;113(4):870-8.

4. Braeken AP, Lechner L, van Gils FC, Houben RM, Eekers D, Ambergen T, et al. The effectiveness of the Screening Inventory of Psychosocial Problems (SIPP) in cancer patients treated with radiotherapy: design of a cluster randomised controlled trial. BMC Cancer. 2009;9(177):1471-2407.

5. Lenzen SA. 2015. Het vier bollen model [The four circle model]. Retrieved from: https://www.zuyd.nl/onderzoek/lectoraten/autonomie-en participatie/ /media/Files/Onderzoek/ Kenniskring\%20Autonomie\%20en\%20participatie\%20van\%20chronisch\%20zieken/Het\%204\%20bollen\%20model\%20voor\%20website\%20121015.pdf

6. Lenzen SA, Daniels R, van Bokhoven MA, van der Weijden T, Beurskens A. What makes it so difficult for nurses to coach patients in shared decision making? A process evaluation. Int J Nurs Stud. 2017;80:1-11.

7. Lenzen SA, Daniels R, van Bokhoven MA, van der Weijden T, Beurskens A. Development of a conversation approach for practice nurses aimed at making shared decisions on goals and action plans with primary care patients. BMC Health Services Research. 2018; 18 [891]. https://doi.org/10.1186/s12913-018-3734-1

8. Elwyn G, Frosch D, Thomson R, Joseph-Williams N, Lloyd A, Kinnersley P, et al. Shared Decision Making: A Model for Clinical Practice. J Gen Intern Med. 2012;27(10):1361-7.

9. Elwyn G, Lloyd A, May C, van der Weijden T, Stiggelbout A, Edwards A, et al. Collaborative deliberation: A model for patient care. Patient Educ Couns. 2014;97(2):158-64.

10. Scobbie L, Dixon D, Wyke S. Goal setting and action planning in the rehabilitation setting: development of a theoretically informed practice framework. Clin Rehabil. 2011;25(5):468-82.

11. Zamanzadeh V, Valizadeh L, Tabrizi FJ, Behshid M, Lotfi M. Challenges associated with the implementation of the nursing process: A systematic review. Iran J Nurs Midwifery Res. 2015;20(4):4119.

12. Gordon M. Nursing diagnosis: process and application. St. Louis, US: Mosby; 1993.

13. Organization WH. International Classification of Functioning, Disability and Health (ICF) 2001.

14. Herdman HT, Kamitsuru Se. NANDA International Nursing Diagnoses: Definitions and Classification 20182020. Thieme, editor. New York, Stuttgart, Delhi 2017.

15. Moorhead S, Swanson E, Johnson M, Maas ML. Nursing Outcome Classification: Elsevier; 2018.

16. Butcher HK, Bulechek GM, Dochterman JM, Wagner CM. Nursing Interventions Classification (NIC). St. Louis, Missouri, US: Elsevier; 2018.

17. Council for Health and Society of the Netherlands. Zonder context geen bewijs: over de illusie van evidence-based practice in de zorg [No evidence without context. About the illusion of evidence-based practice in healthcare]. The Hague; 2017.

18. Felder M, Meerding WJ. Een toekomst voor Evidence-Based Medicine? [A future for Evidence-Based Medicine?] Achtergrondstudie bij het advies "Zonder context geen bewijs. Over de illusie van evidencebased practice in de zorg." [Background study for the report "No evidence without context. About the illustion of evidence-based practice in healthcare]. The Hague: Council for Health and Society of the Netherlands; 2017.

19. Brives C, Le Marcis F, Sanabria E. What's in a Context? Tenses and Tensions in Evidence-Based Medicine. Med Anthropol. 2016;35(5):369-76.

20. Hargraves I, Kunneman M, Brito JP, Montori VM. Caring with evidence based medicine. Bmj. 2016;353:i3530. 
21. van der Weele G, Burgers J. Hulp van NHG-Standaarden bij gedeelde besluitvorming. [How NHGstandards can help with shared decision making] Huisarts en Wetenschap. 2018. Retrieved from: https://www.henw.org/artikelen/hulp-van-nhg-standaarden-bij-gedeelde-besluitvorming

22. Hunter L, Emerald E, Martin G. Pasts, Presents and Futures: An Overview of the Action Research Family. Participatory Activist Research in the Globalised World: Social Change Through the Cultural Professions. Dordrecht: Springer Netherlands; 2013. p. 15-32.

23. Dickens L, Watkins K. Action Research: Rethinking Lewin. 1999; https://doi.org/10.1177/ 1350507699302002.

24. Koshy E, Koshy V, waterman H. Action Research in Healthcare. London, California, New Delhi, Singapore: Sage Publications; 2011.

25. Bodorkós B. Innovation in rural development, networking skills for professional master students, module; action research-techniques Gödöllő: SZENT ISTVÁN UNIVERSITY; 2010-2015.

26. Grol R, Wensing M, Eccles M, Davis D. Improving Patient Care: The Implementation of Change in Health Care. West Sussex: John Wiley \& Sons, Ltd; 2013.

27. Munten G, van den Bogaard J, Cox K, Garretsen H, Bongers I. Implementation of evidence-based practice in nursing using action research: a review. Worldviews Evid Based Nurs. 2010;7(3):135-57.

28. Moore GF, Audrey S, Barker M, Bond L, Bonell C, Hardeman W, et al. Process evaluation of complex interventions: Medical Research Council guidance. BMJ. 2015;350:h1258.

29. Craig P, Dieppe P, Macintyre S, Michie S, Nazareth I, Petticrew M. Developing and evaluating complex interventions: the new Medical Research Council guidance. BMJ. 2008;337.

30. Abma TA, Nierse CJ, Widdershoven GA. Patients as partners in responsive research: methodological notions for collaborations in mixed research teams. Qual Health Res. 2009;19(3):401-15.

31. Hunter DJ, Kieslich K, Littlejohns P, Staniszewska S, Tumilty E, Weale A, et al. Public involvement in health priority setting: future challenges for policy, research and society. J Health Organ Manag. 2016;30(5):796-808.

32. National Institute for Health Research. Involve. Retrieved from: http://www.invo.org.uk/

33. Atieno OP. An analysis of the strengths and limitation of qualitative and quatitative research paradigms problems of education in the 21th century. 2009; 13. Retrieved from: http://www.scientiasocialis.lt/pec/files/pdf/Atieno_Vol.13.pdf

34. Korstjens I, Moser A. Series: Practical guidance to qualitative research. Part 4: Trustworthiness and publishing. Eur J Gen Pract. 2018;24(1):120-4.

35. Lincoln YS, Guba EG. Naturalistic Inquiry. Newburry Park: Sage; 1985.

36. Anney VN. Ensuring the Quality of the Findings of Qualitative Research: Looking at Trustworthiness Criteria JETERAPS. 2014;5(2):272-81.

37. Elwyn G, Barr PJ, Grande SW, Thompson R, Walsh T, Ozanne EM. Developing CollaboRATE: A fast and frugal patient-reported measure of shared decision making in clinical encounters. Patient Educ Couns. 2013;93(1):102-7.

38. Kriston L, Scholl I, Holzel L, Simon D, Loh A, Harter M. The 9-item Shared Decision Making Questionnaire (SDM-Q-9). Development and psychometric properties in a primary care sample. Patient Educ Couns. 2010;80(1):94-9.

39. Greenhalgh T, Howick J, Maskrey N. Evidence based medicine: a movement in crisis? Bmj. 2014;348:g3725.

40. Saunders $\mathrm{H}$, Vehvilainen-Julkunen $\mathrm{K}$. The state of readiness for evidence-based practice among nurses: An integrative review. Int J Nurs Stud. 2016;56:128-40.

41. Melnyk BM, Fineout-Overholt E. Evidence-Based Practice in Nursing \& Healthcare. Philadelphia: Wolters Kluwer Lippincott Williams \& Wilkins; 2014.

42. Stevens KR, Puga F, Low V. The ACE-ERI: an instrument to measure EBP readiness in student and clinical populations. 2012.

43. Stevens K. The Impact of Evidence-Based Practice in Nursing and the Next Big Ideas. The Online Journal of Issues in Nursing 2013;18(2).

44. Friesen-Storms J, Beurskens A, Bours G. Teaching and Implementing Evidence-Based Practice in a Hospital Unit With Secondary Vocational Trained Nurses: Lessons Learned. J Contin Educ Nurs. 2017;48(9):407-12. 
45. Friesen-Storms JH, Moser A, van der Loo S, Beurskens AJ, Bours GJ. Systematic implementation of evidence-based practice in a clinical nursing setting: a participatory action research project. J Clin Nurs. 2015;24(1-2):57-68.

46. van der Goot WE, Keers JC, Kuipers R, Nieweg RMB, de Groot M. The effect of a multifaceted evidencebased practice programme for nurses on knowledge, skills, attitudes, and perceived barriers: A cohort study. Nurse Educ Today. 2018;63:6-11.

47. Regieraad kwaliteit van zorg [Quality of Care Council]. Richtlijn voor Richtlijnen: 20 criteria voor het ontwikkelen en implementeren van een klinische richtlijn [Guideline for guidelines: 20 criteria for the development and implementation of a clinical guideline]. Den Haag; 2012.

48. Institute of Medicine. Clinical Practice Guidelines We Can Trust. Washington DC: The National Academies Press; 2011.

49. Verpleegkundigen en verzorgenden 2020 [Nurses and carers]. Beroepsprofiel verpleegkundige [Professional profile nurses]. Utrecht: V\&V20202; 2012.

50. Beurskens S, Baarends E, Driessen I, Schoot T, Geerbex A, Geraets J, et al. Evidence based practice in het buitenschools leren: enkele uitgangspunten [Evidence-based practice in learning outside school]. Onderwijs en gezondheidszorg. 2008;32(3):23-6.

51. Baarends E, Beurskens S, Van Der Klink M. Evidence Based Practice bij gezondheidszorgprofessionals in het HBO: Inleiding Operationalisatie van het eindniveau van de bachelor [Evidence-based practice for health care professionals in the bachelor education: Introduction to the operationalisation of the end level in the bachelor]. Onderwijs en gezondheidszorg. 2013(4):17-21.

52. Verkerk EW, Huisman-de Waal G, Vermeulen H, Westert GP, Kool RB, van Dulmen SA. Low-value care in nursing: A systematic assessment of clinical practice guidelines. Int J Nurs Stud. 2018;87:34-9.

53. Saunders H. Translating knowledge into best practice care bundles: a pragmatic strategy for EBP implementation via moving postprocedural pain management nursing guidelines into clinical practice. J Clin Nurs. 2015;24(13-14):2035-51.

54. Zwakhalen SMG, Hamers JPH, Metzelthin SF, Ettema R, Heinen M, de Man-Van Ginkel JM, et al. Basic nursing care: The most provided, the least evidence based - A discussion paper. J Clin Nurs. 2018;27(1112):2496-505.

55. Sackett DL, et al. Introduction. 2nd ed. Edinburgh: Churchill Livingstone; 2000. 1-12 p.

56. Friesen-Storms JHHM, Bours GJJW, Weijden Tvd, Beurskens AJHM. Shared decision making in chronic care in the context of evidence based practice in nursing. Int J Nurs Stud. 2015;52(1):393-402.

57. Melnyk BM, Fineout-Overholt E. Consumer preferences and values as an integral key to evidence-based practice. Nurs Adm Q. 2006;30(2):123-7.

58. Satterfield JM, Spring B, Brownson RC, Mullen EJ, Newhouse RP, Walker BB, et al. Toward a transdisciplinary model of evidence-based practice. Milbank Q. 2009;87(2):368-90.

59. Clark NM, Nelson BW, Valerio MA, Gong ZM, Taylor-Fishwick JC, Fletcher M. Consideration of Shared Decision Making in Nursing: A Review of Clinicians' Perceptions and Interventions. Open Nurs J. 2009;3:65-75.

60. Zoffmann V, Harder I, Kirkevold M. A Person-Centered Communication and Reflection Model: Sharing Decision-Making in Chronic Care. Qual Health Res. 2008;18(5):670-85.

61. Coulter A, Entwistle VA, Eccles A, Ryan S, Shepperd S, Perera R. Personalised care planning for adults with chronic or long-term health conditions. Cochrane Database Syst Rev. 2015(3):Cd010523.

62. Vandvik PO, Brandt L, Alonso-Coello P, Treweek S, Akl EA, Kristiansen A, et al. Creating clinical practice guidelines we can trust, use, and share: a new era is imminent. Chest. 2013;144(2):381-9.

63. McCartney M, Treadwell J, Maskrey N, Lehman R. Making evidence based medicine work for individual patients. Bmj. 2016;353:i2452.

64. Hargraves I, LeBlanc A, Shah ND, Montori VM. Shared Decision Making: The Need For Patient-Clinician Conversation, Not Just Information. Health affairs (Project Hope). 2016;35(4):627-9.

65. van Dulmen SA, Lukersmith S, Muxlow J, Santa Mina E, Nijhuis-van der Sanden MW, van der Wees PJ. Supporting a person-centred approach in clinical guidelines. A position paper of the Allied Health Community - Guidelines International Network (G-I-N). Health Expect. 2015;18(5):1543-58. 
66. Kennisbank richtlijnontwikkeling [Knowledge base guideline development]. Tool: Richtlijnen en shared decision making in de praktijk [Tool: Guidelines and shared decision making in clinical practice]: IQ Healthcare; 2013. Retrieved from: https://www.zorginzicht.nl/kennisbank/Documents/ Tool\%20Richtlijnen\%20en\%20shared\%20decision\%20making\%20in\%20de\%20praktijk.pdf

67. Stacey D, Légaré F, Lewis K, Barry MJ, Bennett CL, Eden KB, et al. Decision aids for people facing health treatment or screening decisions. Cochrane Database Syst Rev. 2017(4).

68. Guyatt G, Oxman AD, Akl EA, Kunz R, Vist G, Brozek J, et al. GRADE guidelines: 1. Introduction-GRADE evidence profiles and summary of findings tables. J Clin Epidemiol. 2011;64(4):383-94.

69. Edwards A, Hood K, Matthews E, Russell D, Russell I, Barker J, et al. The Effectiveness of One-to-one Riskcommunication Interventions in Health Care:A Systematic Review. Med Decis Making. 2000;20(3):290-7.

70. Edwards AG, Naik G, Ahmed H, Elwyn GJ, Pickles T, Hood K, et al. Personalised risk communication for informed decision making about taking screening tests. Cochrane Database Syst Rev. 2013(2):Cd001865.

71. Ott KM, Haddock KS, Fox SE, Shinn JK, Walters SE, Hardin JW, et al. The Clinical Nurse Leader: impact on practice outcomes in the Veterans Health Administration. Nursing economic\$. 2009;27(6):363-70, 83; quiz 71.

72. Jukkala A, Greenwood R, Motes T, Block V. Creating innovative clinical nurse leader practicum experiences through academic and practice partnerships. Nursing Educ Perspect. 2013;34(3):186-91.

73. Garner BL, Metcalfe SE, Hallyburton A. International collaboration: A concept model to engage nursing leaders and promote global nursing education partnerships. Nurse Educ Pract. 2009;9(2):102-8.

74. Saunders H, Vehvilainen-Julkunen K. Nurses' Evidence-Based Practice Beliefs and the Role of EvidenceBased Practice Mentors at University Hospitals in Finland. Worldviews Evid Based Nurs. 2017;14(1):3545.

75. Stetler CB, Ritchie JA, Rycroft-Malone J, Charns MP. Leadership for evidence-based practice: strategic and functional behaviors for institutionalizing EBP. Worldviews Evid Based Nurs. 2014;11(4):219-26.

76. Stuurgroep bachelor of nursing 2020 [Steering group bachelor of nursing 2020]. Bachelor Nursing 2020: Een toekomstbestendig opleidingsprofiel 4.0 [Bachelor of nursing 2020: A future proof educational profile]. Landellijk Overleg Opleiding Verpleegkundigen, 2015.

77. Friesen-Storms JHHM, Bours GJJW, Snijders ICG, van der Weijden T, Jie K-SG, Beurskens AJHM. A conversation approach based on shared goal-setting and shared decision-making for nurses in cancer aftercare: A developmental study. Eur J Oncol Nurs. 2018;35:107-16.

78. Friesen-Storms, JHHM, Bours G, Quadvlieg-Delnoy D, Moser A, Heijmans J, van der Weijden T, Beurskens AJHM, Jie KSG. There is more to learn from stories of lymphoma survivor's to further improve aftercare planning: A narrative inquiry. Submitted August 2018.

79. Klaassen L, Friesen S, J.H.H.M., Bours GJJW, Dirksen C, Boersma L, Hoving C. Perceived Facilitators and Barriers for Healthcare Professionals to Adopting a Patient Decision Aid for Choosing Breast Cancer Aftercare: a cross-sectional study. Submitted November 2018.

80. Howell D, Molloy S, Wilkinson K, Green E, Orchard K, Wang K, et al. Patient-reported outcomes in routine cancer clinical practice: a scoping review of use, impact on health outcomes, and implementation factors. Ann Oncol. 2015;26(9):1846-58.

81. McGrail K, Bryan S, Davis J. Let's all go to the PROM: the case for routine patient-reported outcome measurement in Canadian healthcare. Healthcare Papers. 2011;11(4):8-18; discussion 55-8.

82. Snyder CF, Aaronson NK. Use of patient-reported outcomes in clinical practice. Lancet. 2009;374(9687):369-70.

83. Basch E, Artz D, lasonos A, Speakman J, Shannon K, Lin K, et al. Evaluation of an online platform for cancer patient self-reporting of chemotherapy toxicities. J Am Med Inform Assoc. 2007;14(3):264-8.

84. Nicklasson M, Elfstrom ML, Olofson J, Bergman B. The impact of individual quality of life assessment on psychosocial attention in patients with chest malignancies: a randomized study. Support Care Cancer. 2013;21(1):87-95.

85. Lynch J, Goodhart F, Saunders Y, O'Connor SJ. Screening for psychological distress in patients with lung cancer: results of a clinical audit evaluating the use of the patient Distress Thermometer. Support Care Cancer. 2010;19(2):193-202.

86. Chen J. The impact of routine collection of Patient Reported Outcome Measures on patients, providers and health organizations in an oncologic setting: a rapid review. Sax Institute; 2011. 
87. Berry DL, Blumenstein BA, Halpenny B, Wolpin S, Fann JR, Austin-Seymour M, et al. Enhancing patientprovider communication with the electronic self-report assessment for cancer: a randomized trial. J Clin Oncol. 2011;29(8):1029-35.

88. Snyder CF, Blackford AL, Wolff AC, Carducci MA, Herman JM, Wu AW. Feasibility and value of PatientViewpoint: a web system for patient-reported outcomes assessment in clinical practice. Psych Oncol. 2013;22(4):895-901.

89. Howell D, Hack TF, Green E, Fitch M. Cancer distress screening data: Translating knowledge into clinical action for a quality response. Palliat Support Care. 2013;12(1):39-51.

90. Basch E, Snyder C. Overcoming barriers to integrating patient-reported outcomes in clinical practice and electronic health records. Ann Oncol. 2017;28(10):2332-3.

91. Huber M, van Vliet M, Giezenberg M, Winkens B, Heerkens Y, Dagnelie PC, et al. Towards a 'patientcentred' operationalisation of the new dynamic concept of health: a mixed methods study. BMJ Open. 2016;6(1).

92. Silver JK, Raj VS, Fu JB, Wisotzky EM, Smith SR, Kirch RA. Cancer rehabilitation and palliative care: critical components in the delivery of high-quality oncology services. Support Care Cancer. 2015;23(12):363343.

93. Holt KA, Mogensen O, Jensen PT, Hansen DG. Goal setting in cancer rehabilitation and relation to quality of life among women with gynaecological cancer. Acta Oncol. 2015;54(10):1814-23.

94. Ha C, Reddy A, Tavel L, Bruera E. Discussing goals of care for a delirious advanced cancer patient in the hospice setting. J Palliat Med. 2013;16(10):1313-6.

95. Douglas SL, Daly BJ, Meropol NJ, Lipson AR. Patient (Pt) and oncologist (MD) discordance in goals of care in end of life (EOL) decision making. J Clin Oncol 36 2018;36 (suppl; abstr 10007).

96. Integraal Kankercentrum Nederland [Comprehensive Cancer Centre the Netherlands ] (IKNL). 2011. Richtlijn Herstel na Kanker [Guideline Cancer survivorship care]. Retrieved from: http://www.oncoline.nl/herstel-na-kanker.

97. Visserman EA, Gijsen BCM, Blaauwbroek HG. 2014. Zorgstandaard Kanker [Care Standard for Cancer]. Leven met Kanker [Living with cancer], IKNL, KWF Kankerbestrijding, Utrecht.

98. Gilligan T, Coyle N, Frankel RM, Berry DL, Bohlke K, Epstein RM, Finlay E, Jackson VA, Lathan CS, Loprinzi CL, Nguyen LN, Seige Cl. Baile WF. Patient-Clinician Communication: American Societv of Clinical Oncology Consensus Guideline. J Clin Oncol. 2017; 35 (31): 3618-3632. DOI: 10.1200/JCO.2017.75.2311 Retrieved from: https://ascopubs.org/doi/pdf/10.1200/JCO.2017.75.2311

99. Makoul G, Clayman ML. An integrative model of shared decision making in medical encounters. Patient Educ Couns. 2006;60(3):301-12.

100. Howell DD. Supported self-management for cancer survivors to address long-term biopsychosocial consequences of cancer and treatment to optimize living well. Curr Opin Support Palliat Care. 2018.

101 Howell D, Harth T, Brown J, Bennett C, Boyko S. Self-management education interventions for patients with cancer: a systematic review. Support Care Cancer. 2017;25(4):1323-55.

102. Wagner EH, Bennett SM, Austin BT, Greene SM, Schaefer JK, Vonkorff M. Finding common ground: patient-centeredness and evidence based chronic illness care J Altern Complement Med. 2005;11(1):S715.

103. Vermunt N, Harmsen M, Westert GP, Olde Rikkert MGM, Faber MJ. Collaborative goal setting with elderly patients with chronic disease or multimorbidity: a systematic review. BMC geriatrics. 2017;17(1):167.

104. van de Pol MH, Fluit CR, Lagro J, Slaats YH, Olde Rikkert MG, Lagro-Janssen AL. Expert and patient consensus on a dynamic model for shared decision-making in frail older patients. Patient Educ Couns. 2016;99(6):1069-77.

105. Rose A, Rosewilliam S, Soundy A. Shared decision making within goal setting in rehabilitation settings: A systematic review. Patient Educ Couns. 2017;100(1):65-75.

106. Elwyn G, Durand MA, Song J, Aarts J, Barr PJ, Berger Z, et al. A three-talk model for shared decision making: multistage consultation process. Bmj. 2017;359:j4891.

107. Groen van de Ven L, Smits C, Elwyn G, Span M, Jukema J, Eefsting J, et al. Recognizing decision needs: first step for collaborative deliberation in dementia care networks. Patient Educ Couns. 2017;100(7):1329-37. 
108. Monterosso L, Taylor K, Platt V, Lobb E, Krishnasamy M, Musiello T, et al. A qualitative study of the posttreatment experiences and support needs of survivors of lymphoma. Eur J Oncol Nurs. 2017;28:62-8.

109. Burton J. History of lymphoma. JAMA Oncol. 2018;4(3):292.

110. Nekhlyudov L. Survivorship Care Plan: We've Got the Tool-It's Time to Fix the Process. J Natl Cancer Inst. 2018; 110(12) doi: 10.1093/jnci/djy077

111. ASCO American Society of Clinical Oncology. Providing survivorship care in practice. Retrieved from: https://www.asco.org/practice-guidelines/cancer-care-initiatives/preventionsurvivorship/survivorship/survivorship-5

112. Aguinaga S, Ehlers DK, Cosman J, Severson J, Kramer AF, McAuley E. Effects of physical activity on psychological well-being outcomes in breast cancer survivors from pre-diagnosis to post-treatment survivorship. Psych Oncol. 2018.

113. Jansen D, Spreeuwenberg P, Heijmans M. Ontwikkelingen in de zorg voor chronisch zieken [Developments in the care for patients with chronic diseases] Rapportage 2012. Utrecht: Nivel, 2012.

114. Heins M, Kloek C, Francke A, Sinnige J, I. S, Korevaar J, et al. Naar een toekomstbestendige nazorg bij kanker: is er ruimte voor een grotere rol van de eerste lijn? [Towards future proof aftercare in oncology: is there space for a larger role of primary care?]Utrecht: Nivel, 2016.

115. van Dongen JJJ. Interprofessional collaboration in primary care teams : development and evaluation of a multifaceted programme to enhance patient-centredness and efficiency. Maastricht: Maastricht University; 2017.

116. Institute of Medicine. Delivering High-Quality Cancer Care: Charting a New Course for a System in Crisis. Levit LA, Balogh EP, Nass SJ, Ganz PA, editors. Washington, DC: The National Academies Press; 2013. 412 p.

117. Hudson SV, Ohman-Strickland PA, Bator A, O'Malley D, Gundersen D, Lee HS, et al. Breast and prostate cancer survivors' experiences of patient-centered cancer follow-up care from primary care physicians and oncologists. J Cancer Surviv. 2016;10(5):906-14.

118. Damschroder LJ, Aron DC, Keith RE, Kirsh SR, Alexander JA, Lowery JC. Fostering implementation of health services research findings into practice: a consolidated framework for advancing implementation science. Implement Sci. 2009;4:50.

119. Tabak RG, Khoong EC, Chambers D, Brownson RC. Bridging Research and Practice: Models for Dissemination and Implementation Research. Am J Prev med. 2012;43(3):337-50.

120. Atun R, de Jongh T, Secci F, Ohiri K, Adeyi O. Integration of targeted health interventions into health systems: a conceptual framework for analysis. Health Policy Plan. 2010;25(2):104-11.

121. Schloemer T, Schroder-Back P. Criteria for evaluating transferability of health interventions: a systematic review and thematic synthesis. Implement Sci. 2018;13(1):88. 


\section{Appendix 8.1}
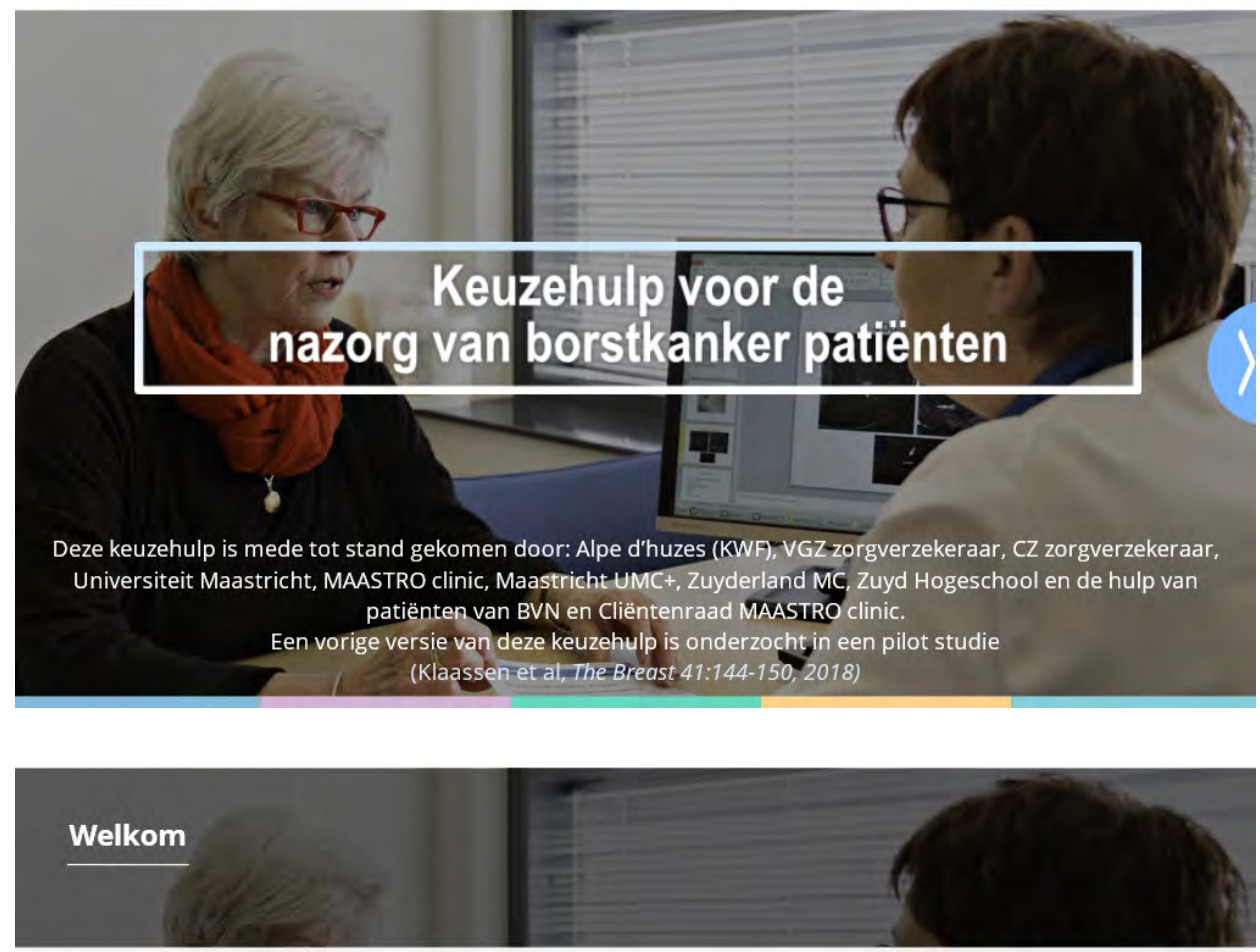

Deze keuzehulp is bedoeld om u te ondersteunen bij het kiezen van een nazorgtraject. Zowel de vorm van de nazorg als de inhoud komen hierbij aan bod. Tijdens het doorlopen van de keuzehulp vult u een aantal vragen in. Als u op de blauwe pijl klikt komt u bij een uitgebreidere instructie over de keuzehulp. 

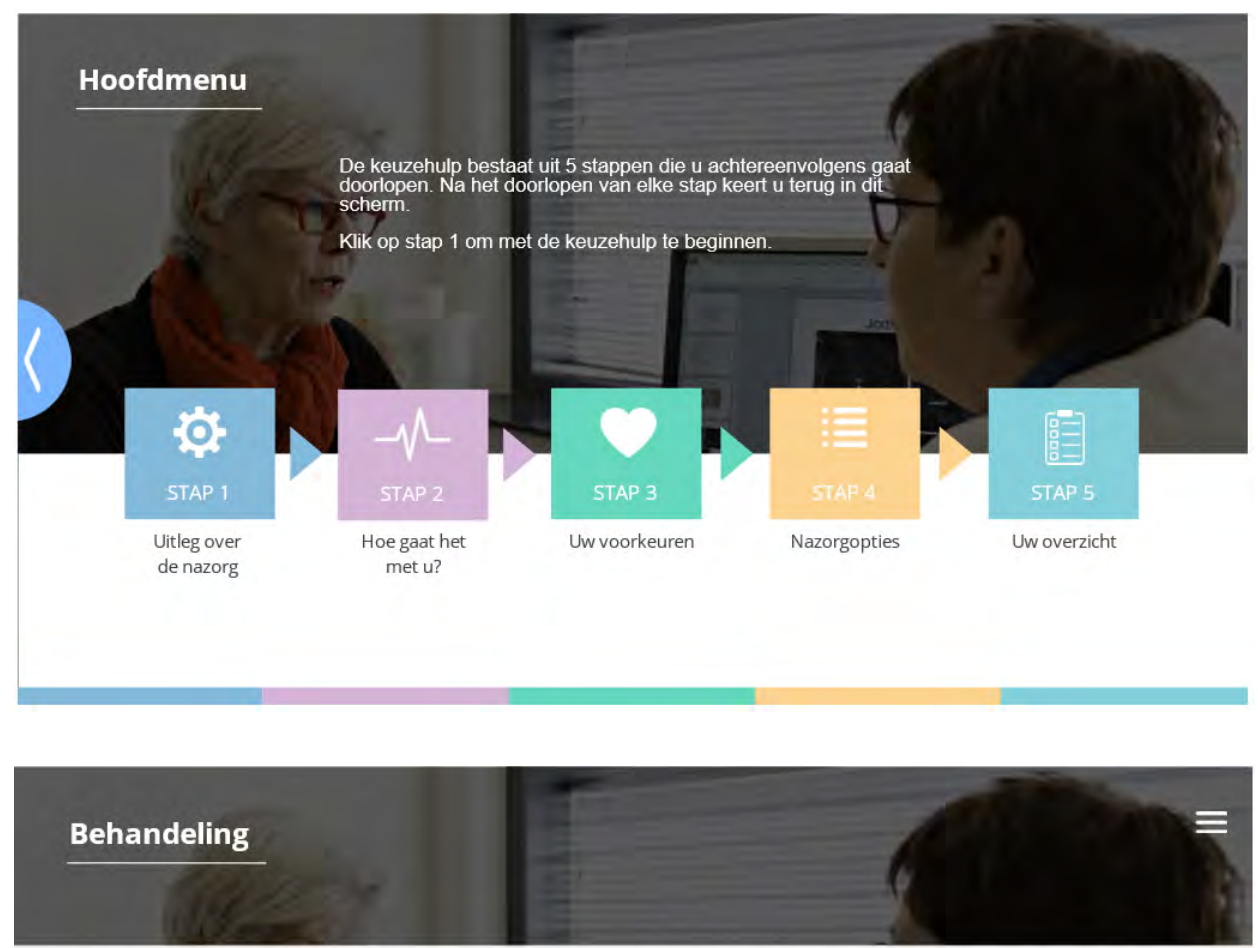

U heeft in de afgelopen periode een behandeling voor borstkanker gehad. Dit kan een operatie, bestraling, chemotherapie, hormonale therapie, doelgerichte therapie of een combinatie hiervan zijn geweest. Misschien wordt u nu nog behandeld.

1

Elke behandeling kan bijwerkingen hebben, hiervoor krijgt u nazorg. Er is altijd een klein risico dat de ziekte terugkomt, hiervoor krijgt u controles.

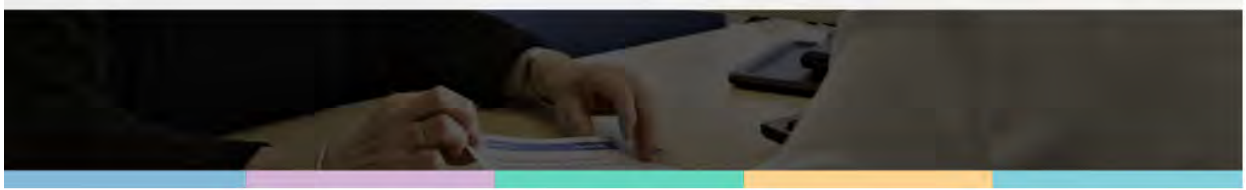




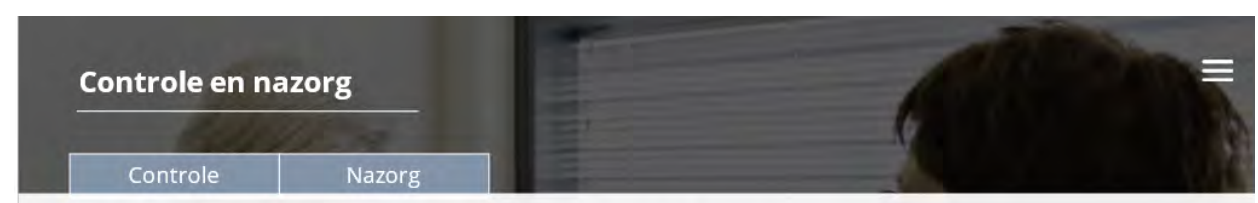

Met de twee tabbladen hierboven (controles en nazorg) wordt het verschil tussen beiden duidelijk.

Lees de informatie rustig door en klik vervolgens op de pijl rechts in het scherm om door te gaan.

Bron: landelijke richtlijn borstkanker, 2018
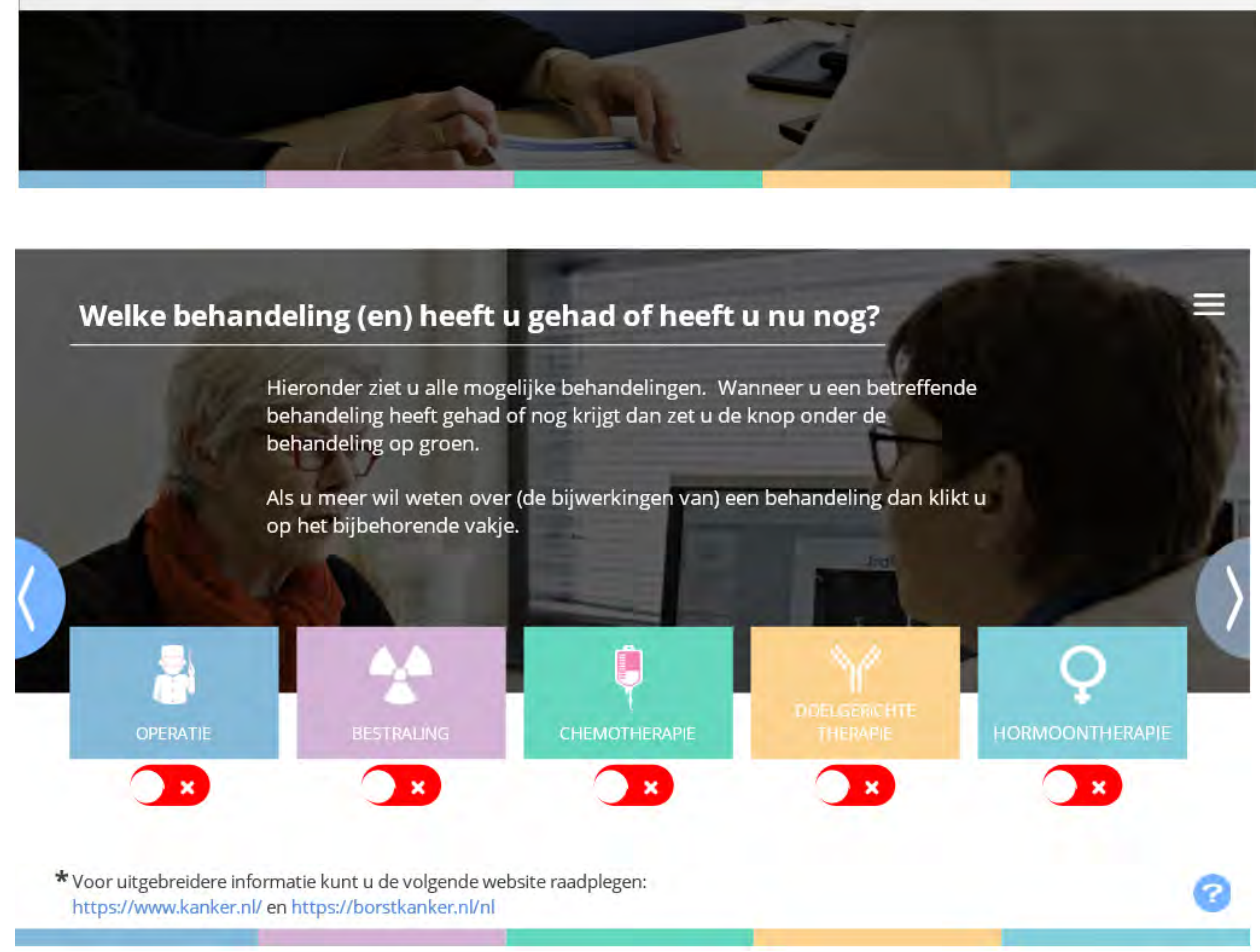

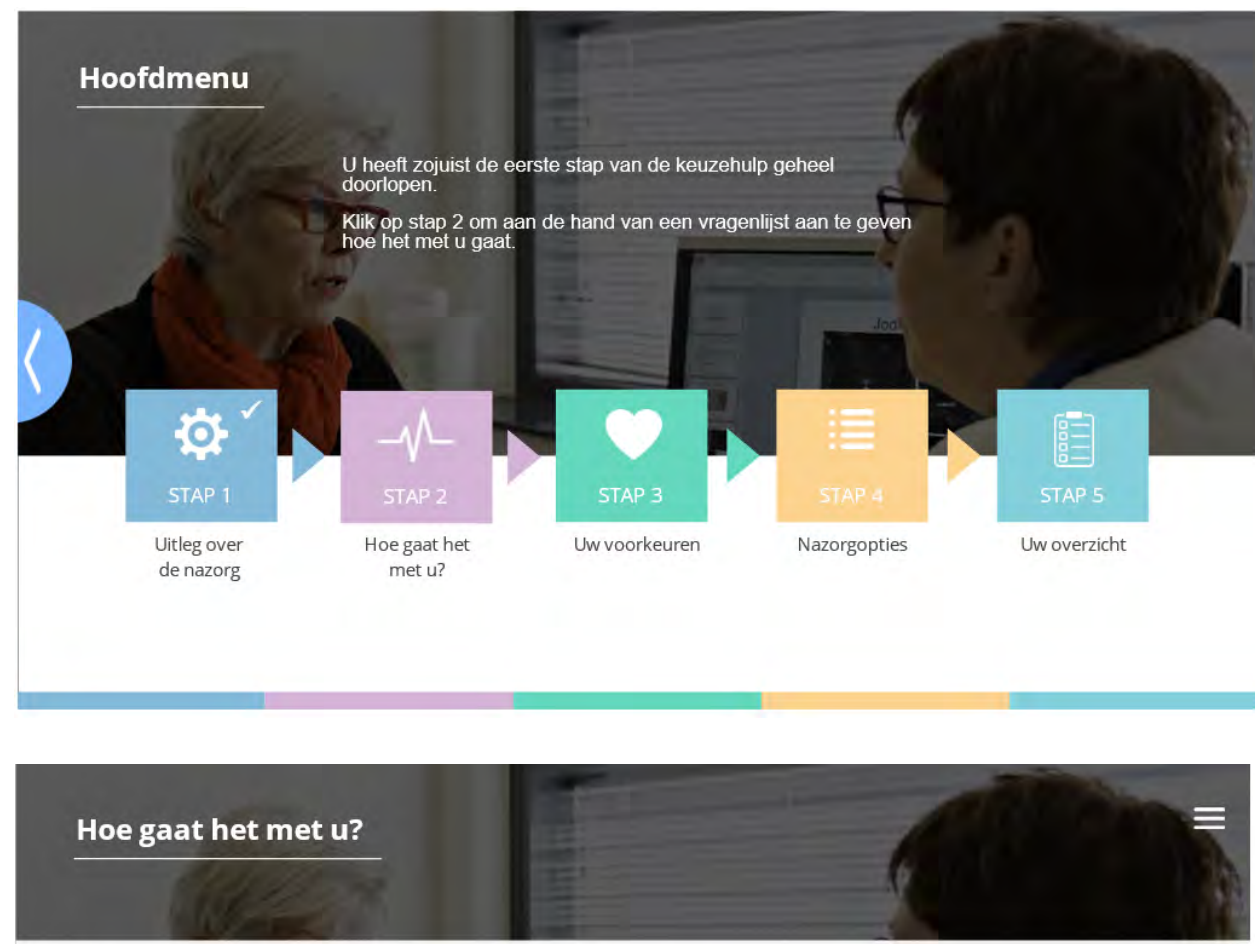

Er wordt nu nagegaan of u nog bijwerkingen heeft van de

behandelingen. $U$ kunt steeds aangeven welke klachten op $u$ van toepassing zijn.

Klachten kunnen op 4 levensgebieden voorkomen:

- mijn gezondheid

- mijn activiteiten

- mijn omgeving

- mijn eigen manier

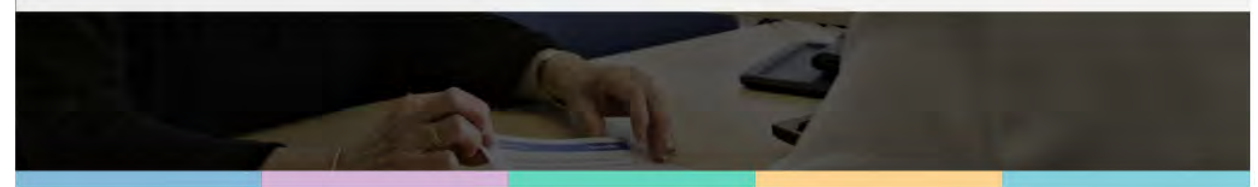




\section{De lastmeter}

Hoeveel last hebt u van problemen, klachten, zorgen?

Vul eerst onderstaande thermometer in.

Klik op de hiernaast afgebeelde thermometer op het cijfer $(0-10)$ dat het best samenvat hoeveel last $u$ de afgelopen week (inclusief vandaag) hebt gehad op lichamelijk,

emotioneel, sociaal en praktisch gebied.

Klik vervolgens op de pijl rechts om door te gaan naar de volgende vragen.

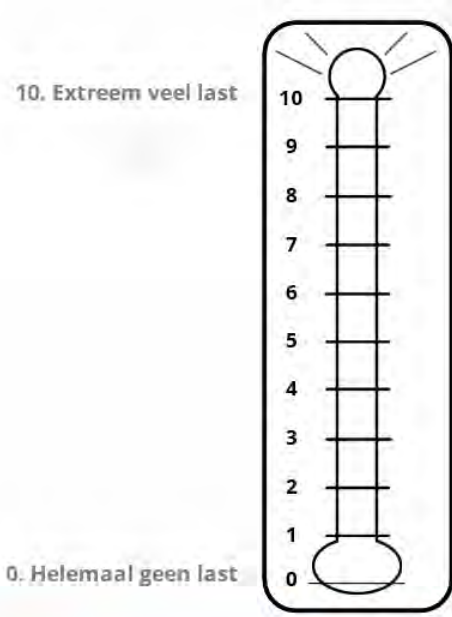

\section{Mijn gezondheid}

Denk aan gezondheidsklachten zoals pijn-, slaapproblemen, vermoeidheid en verminderde conditie. 


\section{Mijn gezondheid}

Wilt $\mathrm{u}$ in deze lijst aangeven of deze klachten/problemen op u van toepassing zijn?

Lichamelijk

Moeheidsklachten

Slaapproblemen

Gewichtsverlies

Slechte eetlust

Duizeligheid

Pijnklachten

Conditieverlies

Verminderde spierkracht

Problemen met uiterlijk

Veranderde urine uitscheiding

Verstopping/obstipatie

Diarree

\section{Ja Soms Nee Lichamelijk}

Problemen met eten

Opgezwollen gevoel

Koorts

Problemen met mondslijmvlies

Misselijkheid

Droge, jeukerige huid

Benauwdheid

Problemen met praten

Veranderd smaakvermogen

Tintelingen in handen/voeten

Oedeem arm

Functieverlies arm
Ja Soms Nee

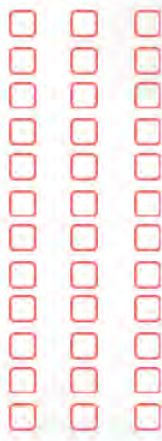

Mijn gezondheid

Wilt $u$ in deze lijst aangeven of deze klachten/problemen op $u$ van toepassing zijn?

\section{Emotioneel}

Piekeren

Rusteloosheid

Gevoelens van eenzaamheid

Gevoelens van verdriet

Gevoelens van verlies

Minder grip op eigen emoties

Verminderd zelfvertrouwen

Angst voor ziekte/behandeling

Gevoelens van neerslachtigheid

Gevoelens van wanhoop

$$
\text { Ja Soms Nee }
$$

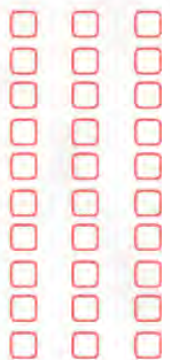

Cognitief

Ja Soms Nee

$\equiv$

Problemen met herinneren van dingen

Concentratie problemen 
Denk aan voor u belangrijke bezigheden.

Welke voor $u$ belangrijke bezigheden gaan er minder goed?

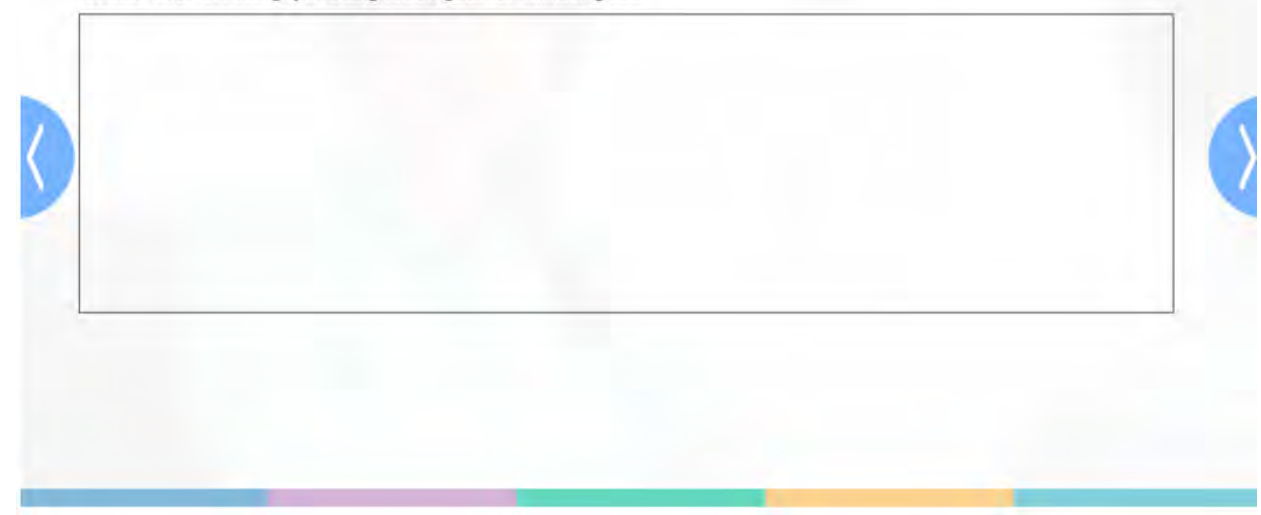

Mijn activiteiten

Wilt $\mathrm{u}$ in deze lijst aangeven of deze klachten/problemen op u van toepassing zijn?

\section{Praktisch}

Beperkingen in dagelijks functioneren Problemen met zorg voor kinderen Problemen met huishouden

Problemen met vervoer Problemen met werk/school/studie Problemen met wassen/aankleden

\section{Ja Soms Nee}

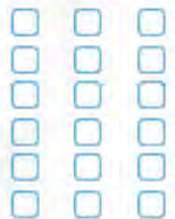


Denk aan de mensen om u heen (familie/ vrienden/ buren) en de omgeving waarin u woont (uw huis, de buurt).

Ervaart u problemen met uw omgeving door uw ziekte en zo ja, welke?

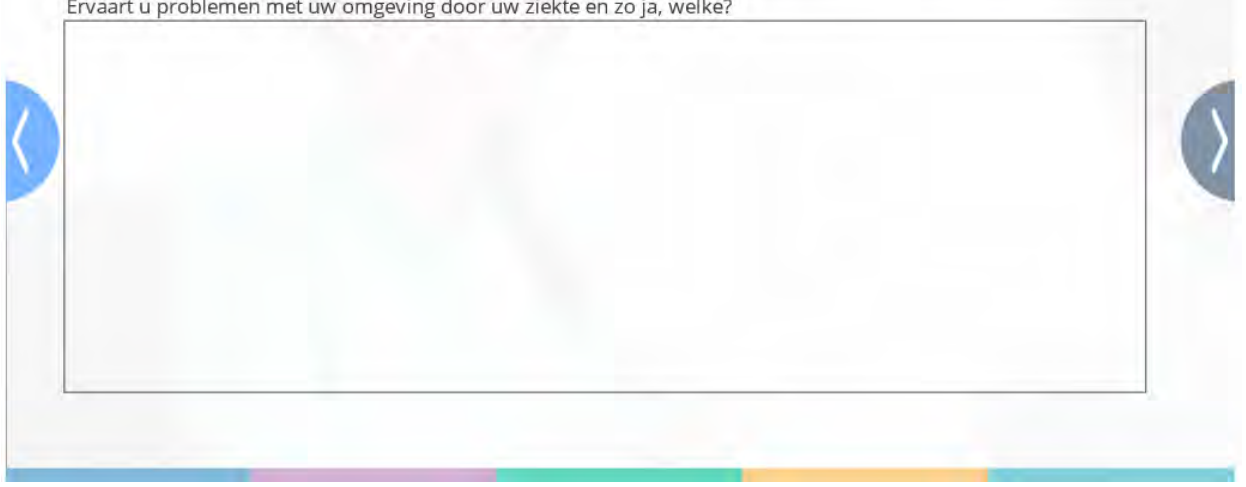

H Mijn omgeving

Wilt $\mathrm{u}$ in deze lijst aangeven of deze klachten/problemen op u van toepassing zijn?

\section{Gezins/sociaal}

Onvoldoende steun door de mensen in uw omgeving Problemen in omgang met uw familie/vrienden

Problemen in omgang met uw kinderen

Problemen in omgang met uw partner

Problemen met intimiteit met uw partner

Minder zin in vrijen

Wenst u voor dit probleem

begeleiding door een

hulpverlener?

\section{Ja Soms Nee NVT Praktisch}

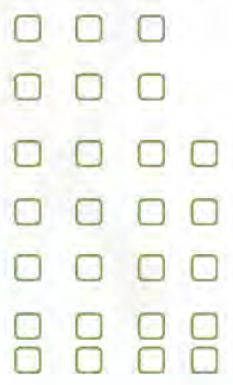

Financiële problemen Problemen met verzekering

Problemen met

wonen/huisvesting
Ja Soms Nee

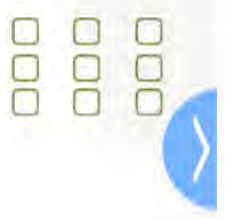




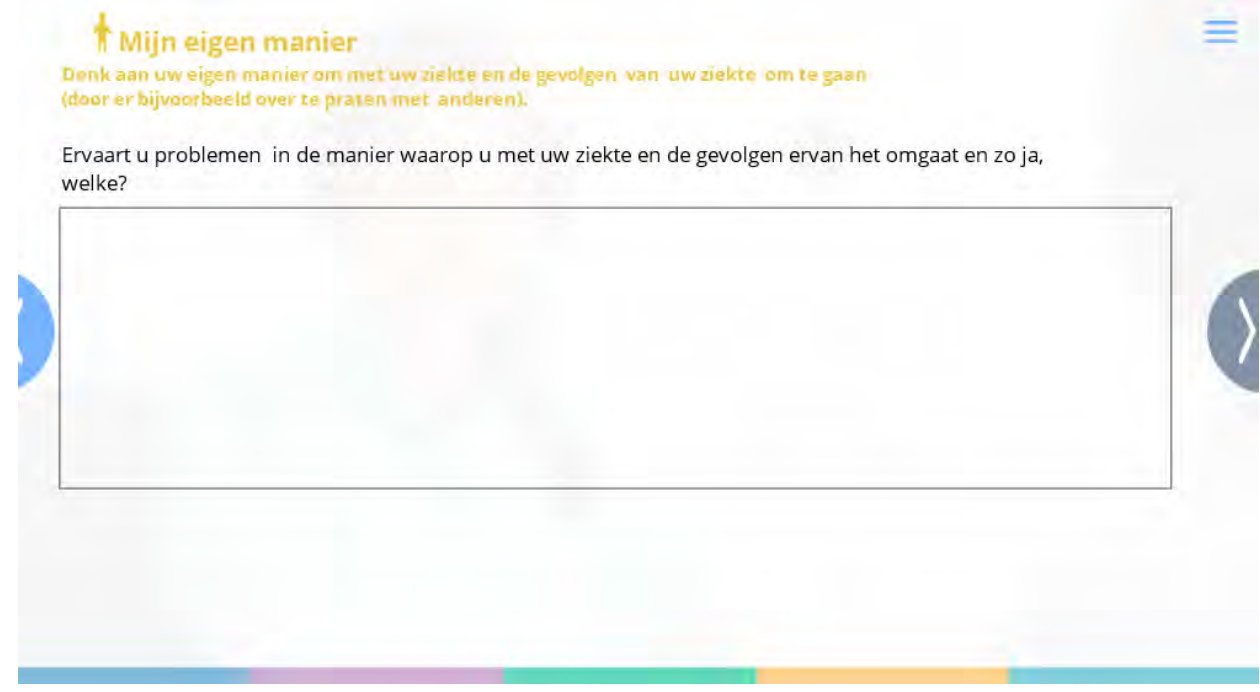

* Mijn aigen manier

$\equiv$

Wilt $u$ in deze lijst aangeven of deze klachten/problemen op u van toepassing zijn?

\section{Ja Soms Nee}

Onvoldoende kunnen praten over het feit dat u kanker heeft Zou u graag met een hulpverlener willen praten?

Vragen over zin van het leven/levensbeschouwing

Veranderingen in vertrouwen in God/geloof

\section{$D$ Overige opmerkingen}

Wat wilt u zelf graag met de zorgverlener bespreken tijdens het nazorggesprek? Dit kunt u hieronder invullen. 


\section{Overzicht vragenlijst}

Onderstaand vindt $u$ een overzicht van de beantwoorde vragen. $\cup$ kunt de verschillende levensgebieden aan de rechterkant aanklikken om de betreffende vragenlijst nogmaals in te zien.

1.

Bij de 'Lastmeter' gaf u een score van $\mathbf{4}$ aan

2.

In de lijst 'Gezondheid' beantwoordde $\mathrm{u}$

11 van de 36 vragen met JA of SOMS

3.

In de lijst 'Activiteiten' beantwoordde $u$

3 van de 6 vragen met JA of SOMS

4.

In de lijst 'Omgeving' beantwoordde u

0 van de 10 vragen met JA of SOMS.

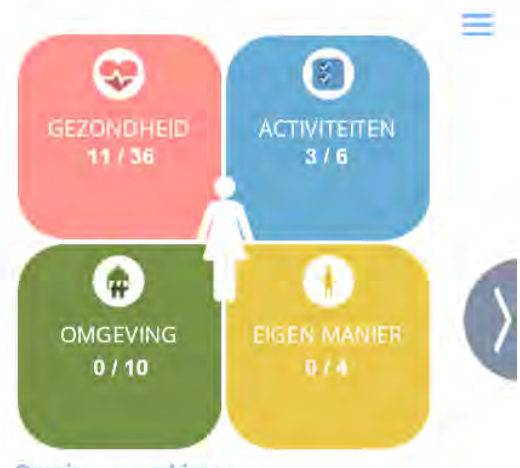

5 .

In de lijst 'Eigen manier' beantwoordde u

0 van de 4 vragen met JA of SOMS

\section{Mijn gezondheid}

Wilt $u$ in deze lijst aangeven of deze klachten/problemen op u van toepassing zijn?

\section{Lichamelijk}

Moeheidsklachten

Slaapproblemen

Gewichtsverlies

Slechte eetlust

Duizeligheid

Pijnklachten

Conditieverlies

Verminderde spierkracht

Problemen met uiterlijk

Veranderde urine uitscheiding

Verstopping/obstipatie

Diarree

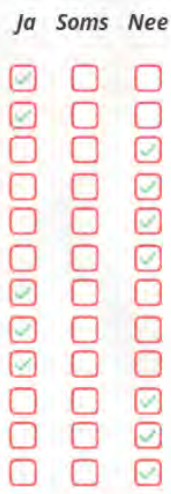

Lichamelijk

Problemen met eten

opgezwollen gevoel

Koorts

Problemen met mondslijmvlies

Misselijkheid

Droge, jeukerige huid

Benauwdheid

Problemen met praten

Veranderd smaakvermogen

Tintelingen in handen/voeten

Oedeem arm

Functieverlies arm
Ja Soms Nee

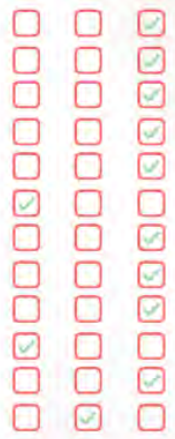




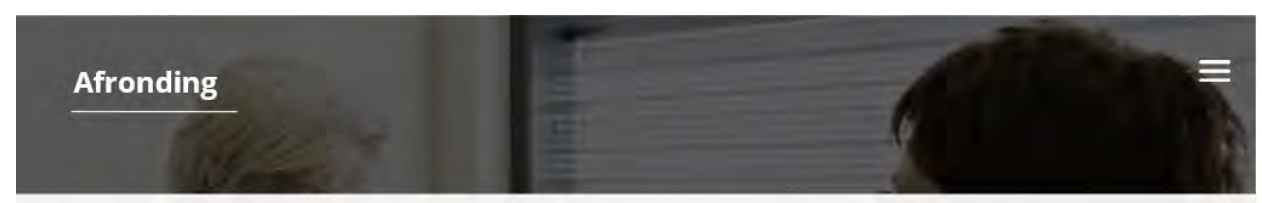

U heeft nu een overzicht gekregen van uw voorkeuren met betrekking tot de vorm van de nazorg, en welke nazorg optie het beste aansluit bij uw voorkeuren. Ook hebt $\mathrm{u}$ een overzicht gekregen van uw antwoorden op vragen over hoe het met u gaat.

1. Deze overzichten kan u met uw zorgverlener bespreken, zodat de zorgverlener samen met u, die vorm van nazorg kan kiezen die het beste bij u past. Samen met u kan nagegaan worden of er nog aanvullende nazorg nodig is, zoals bijvoorbeeld verwijzing naar fysiotherapie of maatschappelijk werk.
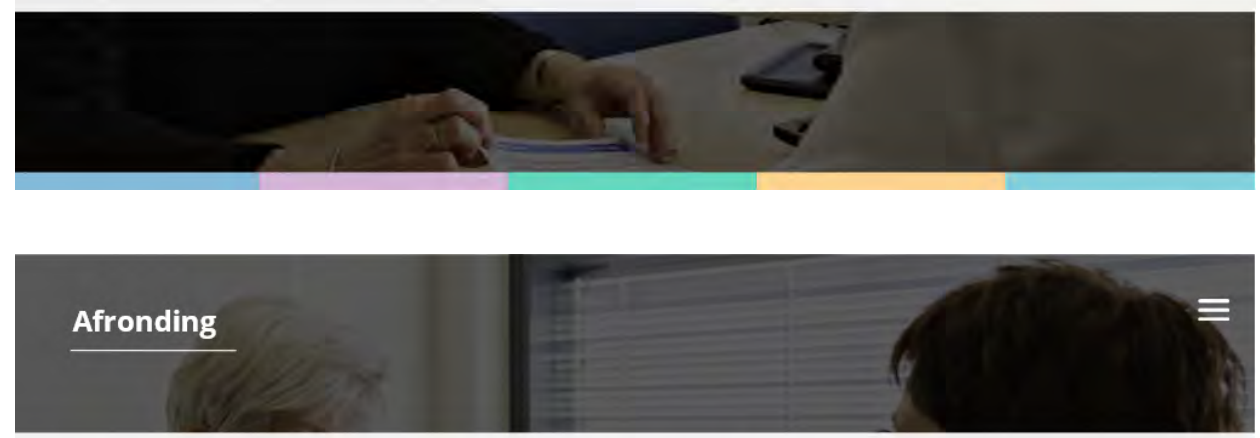

Klik op de knop PRINT UW OVERZICHTEN om uw resultaten te printen of als PDF op te slaan.

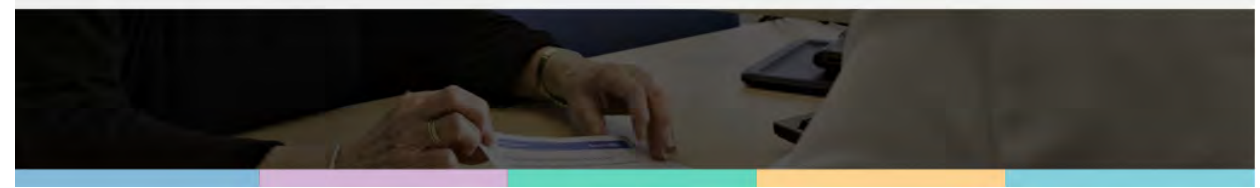


Summary

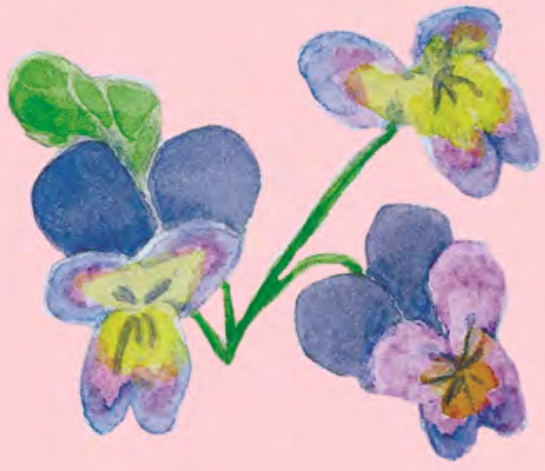





\section{Summary}

The aim of the research reported in this thesis was to gain knowledge about the implementation of evidence-based practice (EBP) in nursing, to find a way to integrate shared decision making (SDM) with EBP in a chronic care environment in nursing, and to develop a strategy for an integrated approach of EBP and SDM in daily nursing practice in the individual aftercare for cancer survivors.

Chapter 1 begins with an introduction to the subject of this research: How to move forward with evidence-based practice in nursing: towards a personalised approach in cancer aftercare. Although EBP is defined as: "the integration of best research evidence with clinical expertise and the patient's values in making decisions about individual patient care", EBP has (so far) mainly focussed on research evidence, while focussing less on the patient's values and clinical expertise. Next to EBP, the communication model SDM has gained interest in health care and nursing as the gold standard for integrating patient values in decision-making process for individual patient care. There is growing insight that suggests SDM should be integrated with EBP. However, how this can be accomplished in nursing is still unclear.

SDM seems particularly relevant in chronic nursing care because, in chronic care involving patients in decision making might support patients in managing their condition in their daily lives. For many people cancer survival, regardless of their disease status, increasingly means living with a chronic condition for which a lot of decisions have to be made about evidence based aftercare. Registered oncology nurses and advanced practice nurses can significantly contribute to the quality of aftercare. Although the relevance of an integrated approach of EBP and SDM in cancer aftercare in nursing is acknowledged, there is no systematic approach developed for daily nursing practice.

To address the different aspects of the aim of this research, this thesis was divided into three parts. For each part of the thesis a research question was formulated:

1. What is the feasibility of implementing evidence-based practice in daily nursing care by interactive and clinically integrated teaching methods?

2. How can shared decision making be integrated with evidence-based practice in chronic nursing care?

3. Which strategy, tailored to the needs of patients and proxies, nurses and other health care professionals, seems worthwhile and feasible to implement an integrated approach of evidence-based practice and shared decision making in cancer aftercare? 
In part 1 we conducted two studies (Chapter 2 and 3) in which EBP was being implemented in nursing. In part 2 (Chapter 4) we discussed the relevance of shared decision making in chronic care and how it can be integrated with evidence-based practice in nursing.

In part 3 (Chapter 5, 6 and 7) we used the knowledge gained from answering research questions 1 and 2 to develop and use EBP and SDM as an integrated approach in a conversation approach for a cancer aftercare consultation.

In Chapter 2 the process and results of implementing EBP using participatory action research on a pulmonary unit of a rural hospital are described. The professionals involved in this study were mainly (secondary) vocationally or medium level trained nurses. Strategies used to implement EBP included a tailored interactive outreach training, and the development and implementation of an evidence-based discharge protocol. The process steps of EBP were adapted to suit the educational level of the participating nurses by, for example, using national evidence-based clinical practice guidelines. A discharge protocol was developed based on the nurses' best-practice mode of working, as well as on evidence from the literature and aspects that emerged in interviews with stakeholders (nurses $n=14$, manager $n=1$, information technology specialists $n=2$, nurses of external care partners $n=2$, patients $n=2$, informal caregivers $\mathrm{n}=2$ ). The summative evaluation showed that nurses did work according to the EBP discharge protocol, but barely recorded their activities in the electronic patient record. Overall, the project stimulated nurses' critical thinking, and nurses positively valued the action research approach, but several nurses remained reluctant about EBP.

This study showed that action research provides an opportunity to empower nurses and to tailor EBP to the practice context. It also showed that applying and implementing EBP is difficult for front-line nurses with limited EBP competencies. It seems necessary to adapt the academic model of EBP to a more pragmatic approach to facilitate introducing EBP into clinical practice for example by using a 'Problem-Intervention'(PI)-question instead of a 'Problem-Intervention-Control-Outcome'(PICO)-question. The use of scientific evidence can be made possible by using pre-appraised evidence.

In Chapter 3 the systematic approach used to teach and implement EBP and the lessons learned are described. The setting of this study was a pulmonary unit of a large teaching hospital with mainly (secondary) vocationally or medium level trained nurses. EBP was systematically implemented using a systematic implementation model. Educational, motivational and organisational strategies were operationalised by developing two main implementation interventions: (1) a tailored interactive outreach training course about EBP and (2) a systems approach, described as "the patient case discussion meeting 
based on EBP principles," was used as a vehicle to implement and embed EBP in daily practice.

We first trained the preceptor nurses of the hospital $(n=10)$ in conducting EBP, subsequently we trained eight nurses of the pulmonary unit.

After the nurses had been trained using the outreach training, patient case discussion meetings were organised. The first patient case discussion meetings led to searching and discussing literature about assessing pain in an elderly patient with impaired communication and subsequently to an implementation proposal for pain measurement.

During the evaluation of the study, nurses mentioned several positive consequences of the training and patient case discussions. Specifically, they reported being able to (1) reflect critically on care (2) formulate a PICO question based on a patient-care problem they had experienced, (3) find English literature and Dutch guidelines related to the problem, and (4) translate the guideline recommendations into routine practice.

Although nurses were able to search for English literature and guidelines, conducting an efficient literature search remained difficult. Critically appraising the literature was considered too difficult for all nurses, even for those with a Bachelor's degree. In addition, nurses formulated large numbers of very specific questions on details of care.

We learned that it is important to simplify the five EBP steps to suit the level of education of nurses. This can be done by formulating PICO questions around major patient care topics, such as pain, and by having a search strategy focusing on Dutch evidence-based clinical practice guidelines and other summarised evidence, such as systematic reviews and critically appraised topics. We advised the hospital management to appoint nurse practitioners or other nurses with a Master's degree to assist the nurses on the ward with the EBP process steps, especially regarding efficiently searching the research literature, and critically appraising evidence.

Chapter 4 discusses the relevance of SDM in chronic care as well as how SDM can be integrated with EBP in nursing. The CINAHL and PubMed digital databases were searched, and a network of experts on evidence-based practice and shared decision making was consulted to include the most relevant papers that underpin our discussion based on previous literature and to ensure that our key points had not already been published elsewhere. The literature search strategy used key words such as '(shared) decision making', 'evidence-based', and 'nurs*' in various combinations.

EBP usually focusses on research evidence (which may be translated into clinical practice guidelines) without considering the individual patient's values. The SDM model seems to be helpful for the integration of individual patients' values in evidence-based practice. Chapter 4 highlights that it is important to always invite the patient to participate in the decision-making process in chronic care and it provides an illustration of how to 
integrate SDM with EBP in chronic care. In addition, the chapter presents 6 different attributes of health care interventions that influence the degree of SDM: the level of research evidence, the presence of intervention options, the burden of side effects, the impact on life style, patient group values and the impact on resources. Patient values become even more important in cases where the level of research evidence is low, there are multiple intervention options, the burden of side effects of the interventions options is high, the impact of the intervention options on life style is high, patient group values are heterogeneous, and there is a high impact on resources.

Next to the attributes of health care interventions, the patient's willingness to participate in SDM, the clinical expertise, knowledge, skills and attitude of the nurse towards SDM, and the context in which the decision making takes place affect the SDM process.

Based on the study described in chapter 4, we concluded that nurses as well as other health care professionals in chronic care should integrate SDM with EBP to deliver patient-centred care.

Chapter 5 describes the development and pre-test of a conversation approach integrating EBP and SDM that can be used by nurses in cancer aftercare to tailor aftercare to the goals, wishes and unmet needs of oncology patients. The setting of the study included several oncology care pathways of a large urban, teaching hospital. We used an iterative developmental process for complex interventions consisting of the following consecutive phases: identifying problems, identifying overall objectives, designing the intervention and pre-testing and adapting the intervention. Problems were first identified during a brainstorm meeting (health care professionals $n=11$, researchers $n=3$ ) and subsequently interviews were conducted (health care professionals $n=9$, patients $n=8$ ). During an expert meeting (health care professionals $n=11$, researchers $n=3$, patients $n=2$ ) overall objectives were formulated based on the results of the problem identification phase. A conversation approach was developed in cooperation with five health care professionals and one patients. Subsequently, the conversation approach was pre-tested (patients $n=11$, nurses $n=3$, nurse practitioner $n=1$ ). All interviews were analysed by directed content analysis.

The main results of the problem identification were: patients were non-systematic and incomplete screened on potential issues and there were caveats in the information patients received and in shared decision-making. The overall objective formulated was: To develop a model for aftercare conversations based on shared goal-setting and decision-making. The developed conversation approach consists of four phases: preparation of the consultation including a questionnaire that included patient reported outcomes, shared goal-setting by means of a tool visualising domains of life, shared care 
planning by using an overview of possible choices in aftercare, a database with health care professionals and a cancer survivorship care plan, and evaluation.

The analysis of the interviews of the pre-test (patients $n=9$, nurses $n=3$, nurse practitioner $n=1$ ) revealed that the conversation approach needs to be flexible and tailored to the patient and practice setting. Using the conversation approach led to more in-depth aftercare consultations. Furthermore, the results of the pre-test revealed important implications and suggestions for implementation, such as tailoring, embedding and digitising the approach within care processes and providing nurses with training and on-the-job coaching. The aftercare conversation approach can be used by nurses and other health care professionals to provide tailored patient-centred evidencebased aftercare. Further evaluation of the process and effect of the conversation approach in practice is needed.

Chapter 6 describes an adoption study that aimed to assess facilitators of and barriers (determinants) to a patient decision aid (PtDA) for aftercare in breast cancer, in which we integrated tools of our conversation approach, to tailor the implementation strategy to. A tailored implementation strategy may improve the likelihood of implementation of the PtDA. Possible important determinants for implementation were divided into three categories: those concerning the PtDA, those concerning the adopter, and those concerning the organisational/socio-political context. The determinants were assessed with the measurement instrument for determinants of innovations (MIDI) questionnaire among breast cancer aftercare health professionals $(n=81)$. For each of the three categories, a backward binominal regression analysis with the intention to adopt the PtDA, as the dependent variable was conducted, to identify significant within-category determinants. Additionally, one binominal regression including all within-category determinants left in the last step of the analyses was performed with intention to adopt the PtDA as the dependent variable.

Overall, relevance of the PtDA for the patient and self-efficacy of the adopter to use the PtDA were significantly positively associated with intention to adopt the PtDA. Significant within-category determinants were: compatibility of the PtDA, outcome expectations of using the PtDA and adherence to the clinical guidelines. We concluded that a tailored implementation strategy should focus on self-efficacy of the adopter and relevance for the patient.

The aim of the study described in chapter 7 was to gain in-depth insight into the personal stories of lymphoma survivors in early aftercare. More specifically, we looked at lymphoma survivors who had been exposed to a best-practice aftercare consultation using the conversation approach. A narrative inquiry was conducted with 22 lymphoma 
survivors in a large teaching hospital in the south of the Netherlands. The participants were recruited after a best-practice aftercare consultation with an advanced practice nurse. The in-depth interviews were analysed by narrative analysis.

Six themes emerged from the narratives: living and dealing with health consequences, coping with work and financial challenges, having a positive outlook and dealing with uncertainty, deriving strength from and experiencing tensions in relationships, getting through tough times in life, and receiving support from health care professionals. Participants had to deal with health consequences like fatigue and lengthy absence(s) from work. Their experiences and strategies for dealing with the consequences of the lymphoma varied. They greatly valued the social support they received, although some social relationships had deteriorated. Apart from the treatment, life events relating to the cancer treatment or their life in general had caused respondents tough times and were part of their life stories. The added value of the aftercare consultation and the need for further consultations was experienced differently. In some cases the consultation had led to additional interventions, while in other cases they had perceived it as rounding off the treatment process.

The stories of lymphoma survivors in early aftercare revealed their experiences of how they coped with various issues in their personal lives. Addressing survivors' social support and self-management capabilities, and building a personal relation(ship) with a patient (as a person rather than just a patient) might help nurses and other health care professionals to better tailor their aftercare.

Chapter 8 presents the thesis general discussion. This final chapter begins with an overview of the main findings, and then discusses several methodological and theoretical considerations and ends with an insight into (possible) implications of this research project for practice, education and research.

We concluded that it is important for nurses to integrate summarised, critically appraised, and translated research, like evidence-based clinical practice guidelines, into evidence-based decision making, and to always invite the patient to get involved in decision making in chronic care. We proposed how to integrate EBP and SDM and we developed a conversation approach (in which both EBP and SDM are integrated) for use in cancer aftercare, which incorporates PROMs and visual tools, and supports goal setting. Using the conversation approach might support providing patients with tailored aftercare.

Looking towards the future, for nursing practice, we recommend adapting the process steps of EBP to suit the level of education of nurses and we suggest using a systematic approach to tailor care to chronic patients. For education, we recommend that nurse educators integrate EBP and SDM and use a conversation approach, like the one we 
developed, in the curriculum. As we experienced major barriers during our research due to a merge of two hospitals, we advise for research to carefully reconsider conducting research on or implementing complex interventions in a dynamic context, such as might exist during a merge. 

Samenvatting

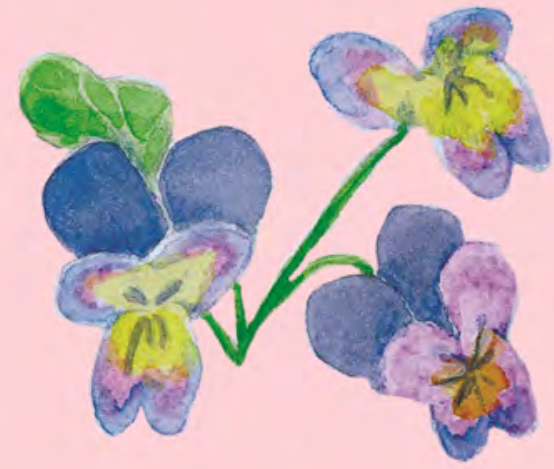





\section{Samenvatting}

De doelstelling van het onderzoek is drieledig: 1 . het verwerven van kennis over de implementatie van evidence-based practice (EBP) binnen de verpleegkunde; 2 . het vinden van een manier om shared decision making (SDM), ook wel gezamenlijke besluitvorming genoemd, te integreren met EBP in de verpleegkundige zorg voor chronisch zieken en 3 . het ontwikkelen van een strategie voor een benadering die EBP en SDM integreert in de verpleegkundige nazorg voor mensen die behandeld zijn voor kanker.

Hoofdstuk 1 beschrijft de inleiding op het onderzoek met als titel: Hoe verder te gaan met evidence-based practice binnen de verpleegkunde: naar een gepersonaliseerde aanpak in de nazorg voor kanker. EBP integreert klinische expertise met het beste externe bewijsmateriaal dat vanuit systematisch onderzoek beschikbaar is, in combinatie met de waarden van de patiënt. EBP heeft zich echter (tot nu toe) voornamelijk gericht op het best beschikbare bewijs en minder op de waarden van de patiënt en klinische expertise. Naast EBP krijgt het model voor gezamenlijke besluitvorming meer belangstelling binnen de gezondheidszorg en binnen de verpleegkunde. Bij dit model ligt de nadruk op het integreren van de waarden van de patiënt in het besluitvormingsproces voor de individuele patiënt. Er is in toenemende mate inzicht dat gezamenlijke besluitvorming geïntegreerd zou moeten worden met EBP. Het is echter nog niet duidelijk hoe dit bereikt kan worden.

Een patiënt met een chronische ziekte staat voor de uitdaging een balans te vinden tussen de eisen van de ziekte en die van het leven, waarbij zelfmanagement centraal staat. Zelfmanagement betekent dat mensen zelf kunnen kiezen in hoeverre zij de regie over het leven in eigen hand willen houden en hoe zij mede richting willen geven aan de manier waarop de beschikbare zorg wordt ingezet. Gezamenlijke besluitvorming door de patiënt en zorgverlener(s) kan bijdragen aan het versterken hiervan. Kanker ontwikkelt zich meer en meer tot een chronische ziekte. Steeds meer mensen die behandeld worden voor kanker ervaren langdurig chronische klachten, zoals vermoeidheid, conditieverlies en angst. Voor deze klachten zijn diverse nazorgmogelijkheden beschikbaar zoals fysiotherapie en oncologische revalidatie. Bij elke persoon moet worden nagegaan welke nazorg het beste past en de voorkeur heeft van de patiënt. Verpleegkundigen kunnen een belangrijke rol vervullen in deze nazorg. Ofschoon het belang van een geïntegreerde benadering van EBP en gezamenlijke besluitvorming in de verpleegkundige nazorg voor kanker wordt erkend, is er geen systematische aanpak ontwikkeld om deze nazorg vorm te geven. 
In deze thesis staan drie onderzoeksvragen centraal, die gerelateerd zijn aan de doelstelling.

1. Wat is de hanteerbaarheid van het implementeren van evidence-based practice in de dagelijkse verpleegkundige zorg door middel van interactieve en in de praktijk geïntegreerde leermethoden?

2. Hoe kan gezamenlijke besluitvorming binnen de verpleegkundige zorg voor mensen met een chronische ziekte geïntegreerd worden met evidence-based practice?

3. Welke strategie, afgestemd op de behoeften van patiënten en hun naasten, verpleegkundigen en andere zorgverleners, lijkt waardevol en hanteerbaar om een geïntegreerde aanpak van evidence-based practice en gezamenlijke besluitvorming in de nazorg voor kanker te implementeren?

In deel 1 zijn twee studies uitgevoerd (Hoofdstuk 2 en 3) waarbij EBP is geïmplementeerd binnen de verpleegkundige zorg. In deel 2 (Hoofdstuk 4) bediscussiëren we het belang van gezamenlijke besluitvorming en hoe deze geïntegreerd kan worden met EBP binnen de verpleegkundige zorg voor mensen met een chronische ziekte. In deel 3 (Hoofdstukken 5, 6 en 7) ontwikkelen we, met behulp van de kennis die is opgedaan bij het beantwoorden van onderzoeksvraag 1 en 2, een gespreksmodel voor een nazorggesprek bij kanker. In dit gespreksmodel zijn EBP en gezamenlijke besluitvorming geïntegreerd.

Hoofdstuk 2 geeft het proces en de resultaten weer van een actieonderzoek dat is uitgevoerd om EBP te implementeren op een longafdeling van een perifeer ziekenhuis. Alle verpleegkundigen op deze afdeling waren middelbaar opgeleide (MBO) verpleegkundigen. Tijdens het onderzoek volgden de verpleegkundigen een interactieve training met betrekking tot EBP en samen met hen ontwikkelden en implementeerden we een evidence-based ontslagprotocol. We vereenvoudigden de veel gebruikte processtappen van EBP om tegemoet te komen aan het opleidingsniveau van de verpleegkundigen. Zo maakten de verpleegkundigen voor het verzamelen van wetenschappelijk bewijs gebruik van nationale evidence-based richtlijnen. Het ontwikkelde ontslagprotocol is gebaseerd op de best-practice manier van werken van de verpleegkundigen, op wetenschappelijk bewijs uit de literatuur en op onderdelen die naar voren kwamen in interviews met belanghebbenden (14 verpleegkundigen, één afdelingsmanager, twee ICT deskundigen, twee externe verpleegkundigen, twee patiënten en twee mantelzorgers). Tijdens de evaluatie van de studie bleek dat de verpleegkundigen wel werkten volgens het ontwikkelde ontslagprotocol, maar ze noteerden de acties die ze ondernomen hadden voor het ontslag van de patiënt zelden in het elektronische patiëntendossier. Door het project gingen de verpleegkundigen 
kritischer denken. Ook waren zij positief over de actiegerichte aanpak van het project. Sommige verpleegkundigen bleven echter terughoudend met betrekking tot EBP.

Deze studie laat zien dat het uitvoeren van actieonderzoek verpleegkundigen kan helpen om EBP te implementeren. Ook laat de studie zien dat het toepassen en implementeren van EBP moeilijk is voor verpleegkundigen met beperkte EBP-competenties. Het lijkt noodzakelijk om het veel gebruikte model van EBP te vertalen naar een meer pragmatische aanpak die het gebruik van EBP in de dagelijkse praktijk vergemakkelijkt. In plaats van het opstellen van 'Probleem-Interventie-Controle-Outcome' (PICO)-vragen, kunnen 'Probleem-Interventie' (PI)-vragen opgesteld worden. Verder kan er gebruik gemaakt worden van wetenschappelijke literatuur die al kritisch beoordeeld en samengevat is in bijvoorbeeld evidence-based richtlijnen of systematische reviews.

Hoofdstuk 3 beschrijft de lessen die naar voren kwamen tijdens een systematische aanpak om EBP te trainen en te implementeren op een longafdeling van een groot opleidingsziekenhuis. Op deze afdeling werkten voornamelijk MBO opgeleide verpleegkundigen. Voor de implementatie van EBP hebben we gebruik gemaakt van een systematisch implementatiemodel, waarbij eerst in kaart werd gebracht in hoeverre de verpleegkundigen EBP toepassen en wat bevorderende en belemmerende factoren voor het gebruik van EBP zijn. Op basis van de resultaten hiervan, hebben we twee belangrijke interventies toegepast om EBP te implementeren: 1. een op maat gemaakte interactieve EBP-training en 2. een patiëntendossier-bespreking waarbij de processtappen van EBP systematisch gevolgd worden, om problemen die men op de afdeling ervaart met de zorg voor een patiënt op te lossen.

Allereerst hebben we de praktijkopleiders van het ziekenhuis $(n=10)$ in EBP getraind en daaropvolgend acht verpleegkundigen van de longafdeling. Na de training hebben we patiëntendossier-besprekingen georganiseerd. In de eerste bespreking stond het meten van pijn bij een geriatrische patiënt met beperkte communicatievaardigheden centraal. De verpleegkundigen hebben een PICO hierover opgesteld en gezocht naar wetenschappelijke literatuur. In een volgend overleg hebben ze de gevonden literatuur teruggekoppeld en uiteindelijk heeft dit op de afdeling geleid tot een voorstel voor pijnassessment.

Tijdens de evaluatie van de studie hebben de verpleegkundigen $(n=8)$ verscheidene positieve gevolgen van de training en de dossierbesprekingen benoemd, zoals: 1 . in staat zijn om kritisch te reflecteren op de zorg; 2. een PICO-vraag op basis van een patiëntprobleem te kunnen opstellen; 3. Engelse literatuur en Nederlandse richtlijnen te kunnen vinden en 4 . de aanbevelingen uit de richtlijnen te kunnen vertalen naar de praktijk. 
Ofschoon de verpleegkundigen in staat waren om Engelse literatuur en richtlijnen te zoeken, was het efficiënt zoeken van literatuur lastig. De verpleegkundigen formuleerden veel zeer specifieke vragen over details in de zorg, waardoor ze lastig literatuur konden zoeken. Ook het kritisch beoordelen van de literatuur was te moeilijk voor de verpleegkundigen, zelfs voor de HBO opgeleide verpleegkundigen. We hebben hiervan geleerd dat het belangrijk is om de vijf stappen van EBP te vereenvoudigen om tegemoet te komen aan het opleidingsniveau van de verpleegkundigen. Dit kan bereikt worden door PICO-vragen rond belangrijke patiëntenproblemen te formuleren, zoals pijn. Of door de zoekstrategie te richten op Nederlandse evidence-based richtlijnen en ander wetenschappelijk bewijs dat al samengevat en kritisch beoordeeld is, zoals systematische reviews en 'Critically Appraised Topics'. Ook hebben we het ziekenhuismanagement geadviseerd te faciliteren dat verpleegkundig specialisten en andere master opgeleide verpleegkundigen de afdelingsverpleegkundigen ondersteunen bij het uitvoeren van de EBP-stappen. Dit is zeker van belang bij het efficiënt zoeken van onderzoeksliteratuur en het kritisch beoordelen ervan.

Hoofdstuk 4 presenteert een discussie van zowel de relevantie van gezamenlijke besluitvorming in de zorg voor mensen met een chronische ziekte als ook hoe het geïntegreerd kan worden met EBP binnen de verpleegkunde. Om de belangrijkste artikelen op dit gebied mee te nemen in de discussie, hebben we de databanken CINAHL en PubMed doorzocht met zoektermen zoals '(shared) decision making', 'evidencebased', and 'nurs*' in diverse combinaties. Ook hebben we experts met betrekking tot EBP en gezamenlijke besluitvorming geraadpleegd.

EBP focust meestal op wetenschappelijk bewijs (dat opgenomen kan zijn in richtlijnen) zonder rekening te houden met individuele patiëntwaarden en klinische expertise. Het model van gezamenlijke besluitvorming kan ondersteunen bij de integratie van individuele patiëntwaarden in evidence-based practice. Verder lichten we in de discussie toe dat het in de chronische zorg altijd van belang is om de patiënt uit te nodigen om deel te nemen aan het besluitvormingsproces. Ook illustreren we hoe gezamenlijke besluitvorming geïntegreerd kan worden met EBP binnen de verpleegkundige zorg voor mensen met een chronische ziekte. We beschrijven zes verschillende kenmerken van interventies met betrekking tot gezondheid die gezamenlijke besluitvorming beïnvloeden: 1 . het niveau van wetenschappelijk bewijs; 2 . de aanwezigheid van interventieopties; 3. de mate van bijwerkingen; 4. de impact op de levensstijl; 5. patiëntwaarden binnen een groep en 6 . de impact op (financiële) middelen. De waarden van de patiënt worden belangrijker wanneer het wetenschappelijk bewijs laag is, er meerdere interventieopties zijn, de mate van bijwerkingen van de interventies 
hoog is, de impact van de interventies op de levensstijl hoog is, patiëntwaarden heterogeen zijn en er een grote impact op (financiële) middelen is.

Naast de kenmerken van de interventies, zijn bij gezamenlijke besluitvorming de bereidheid van de patiënt om deel te nemen aan het besluitvormingsproces en de klinische expertise van de verpleegkundige belangrijk. Ook speelt de context waarbinnen de beslissing plaats vindt een rol.

De conclusie is dat verpleegkundigen en andere zorgverleners in de chronische zorg, gezamenlijke besluitvorming en EBP zouden moeten integreren om patiëntgecentreerde zorg te leveren.

Hoofdstuk 5 beschrijft de ontwikkeling en pre-test van een gespreksmodel, dat EBP en gezamenlijke besluitvorming integreert. De studie heeft plaatsgevonden bij verscheidene oncologische zorgpaden van een groot opleidingsziekenhuis. We hebben een iteratief ontwikkelingsproces voor complexe interventies toegepast om het gespreksmodel te ontwikkelen. Dit ontwikkelingsproces bestond uit de volgende fasen: het vaststellen van problemen, het formuleren van algemene doelen, het ontwerpen van de interventie en het pre-testen en aanpassen van de interventie. Voor het vaststellen van problemen in de nazorg hebben we een brainstormmeeting met belanghebbenden georganiseerd (elf zorgverleners en drie onderzoekers). Vervolgens hebben we belanghebbenden geïnterviewd (negen zorgverleners en acht patiënten behandeld voor kanker). De resultaten van de brainstormmeeting en de interviews zijn vervolgens gebruikt om tijdens een expertmeeting (elf zorgverleners, twee patiënten en drie onderzoekers) doelen voor nazorg te formuleren. Daarna hebben we het nazorggesprek ontworpen met behulp van vijf zorgverleners en één patiënt. Tenslotte hebben we een pre-test van het gespreksmodel uitgevoerd (elf patiënten, drie verpleegkundigen en één verpleegkundig specialist). Alle interviews zijn geanalyseerd met behulp van 'directed' content analyse.

Uit de fase van probleemverheldering, kwam naar voren dat patiënten niet altijd systematisch gescreend werden op de gevolgen van kanker en de kankerbehandeling. Ook kregen ze niet altijd de nodige voorlichting en vond er niet altijd gezamenlijke besluitvorming plaats. Tijdens de expertmeeting werd het volgende doel geformuleerd: het ontwikkelen van een gespreksmodel voor nazorggesprekken gebaseerd op samen doelen opstellen en gezamenlijke besluitvorming. Het ontwikkelde gespreksmodel bestaat uit vier fasen: 1 . voorbereiding van het consult met behulp van een vragenlijst waarin patiënten zelf de gevolgen die ze ervaren van de kanker en de behandeling rapporteren, 2 . het gezamenlijk doelen stellen met behulp van een tool die verschillende levensgebieden visualiseert, 3 . het gezamenlijk opstellen van een zorgplan met behulp 
van een overzicht van mogelijke keuzen in de nazorg, een databank met zorgverleners en een nazorgplan en 4. evaluatie.

Uit de interviews (negen patiënten, drie verpleegkundigen en één verpleegkundig specialist), uitgevoerd tijdens de pre-test van het gespreksmodel, kwam naar voren dat het gespreksmodel flexibel gebruikt moet kunnen worden. Ook moet het aangepast worden aan de patiënt en de praktijksetting. Volgens de deelnemers leidt het gebruik van het gespreksmodel tot meer diepgaande gesprekken met patiënten.

Verder kwamen uit de pre-test belangrijke aanbevelingen voor implementatie naar voren. Zo is het van belang dat het gespreksmodel gedigitaliseerd en ingebouwd wordt in bestaande zorgprocessen. Ook is het belangrijk dat verpleegkundigen getraind worden in het gebruik van het gespreksmodel en dat zij een coaching op de werkplek ontvangen. Het gespreksmodel voor nazorg kan gebruikt worden door verpleegkundigen en andere zorgverleners om op maat, patiëntgerichte nazorg aan te bieden. Het uitvoeren van een proces- en effectevaluatie wordt aanbevolen.

Hoofdstuk 6 beschrijft een adoptie-studie die tot doel heeft het vaststellen van bevorderende en belemmerende factoren (determinanten) bij zorgverleners om een nazorgkeuzehulp te gebruiken bij de nazorg van borstkankerpatiënten. In deze nazorgkeuzehulp hebben we tools van het gespreksmodel verwerkt.

Door de strategieën bij de implementatie te richten op deze determinanten, kan het gebruik van deze nazorgkeuzehulp in de praktijk bevorderd worden. Mogelijke belangrijke determinanten hebben we onderverdeeld in drie categorieën: determinanten van de nazorgkeuzehulp, determinanten van degene die de keuzehulp adopteert en determinanten die behoren bij de organisatie en de sociaal-politieke context. Vervolgens hebben we de determinanten die bij de zorgverleners een rol spelen, vastgesteld met behulp van het meetinstrument voor determinanten van innovaties (MIDI) ( $n=81$ ). Met behulp van regressie analyse zijn de determinanten bepaald die van invloed zijn op de adoptie van de nazorgkeuzehulp door zorgverleners.

Zorgverleners zijn eerder geneigd om de nazorgkeuzehulp te adopteren indien ze denken dat de keuzehulp relevant is voor de patiënt en indien ze verwachten dat ze zelf in staat zijn om deze te kunnen toepassen. Verder zijn belangrijke determinanten: de inpasbaarheid van de keuzehulp in zorgprocessen, de uitkomstverwachtingen ten aanzien van de keuzehulp en het opvolgen van richtlijnen door het gebruik van de keuzehulp.

De conclusie is dat een strategie op maat zich moet richten op verwachting van de zorgverlener om de keuzehulp te kunnen toepassen en op de relevantie voor de patiënt. 
Hoofdstuk 7 presenteert een narratieve studie die tot doel heeft om diepgaand inzicht te verkrijgen in de persoonlijke ervaringen van overlevenden van een maligne lymfoom in de vroege nazorgfase na een best-practice nazorggesprek met een verpleegkundig specialist in opleiding (VSio). Bij dit nazorggesprek is het in hoofdstuk 5 ontwikkelde gespreksmodel gebruikt. We hebben diepte-interviews gehouden met 22 overlevenden van een maligne lymfoom in een groot opleidingsziekenhuis in het zuiden van Nederland. De deelnemers zijn geselecteerd na een best-practice nazorggesprek door de VSio. De interviews zijn geanalyseerd met behulp van een narratieve analyse.

Uit de verhalen kwamen zes thema's naar voren: leven en omgaan met de gevolgen voor de gezondheid; omgaan met werk en met financiële gevolgen; een positief vooruitzicht hebben en omgaan met onzekerheid; kracht halen uit en spanningen ervaren binnen relaties; door moeilijke tijden in het leven heenkomen; en steun ontvangen van zorgverleners. Overlevenden van maligne lymfoom moesten omgaan met de gevolgen van de kanker en de behandeling zoals vermoeidheid en langdurige afwezigheid van werk. Hun ervaringen en strategieën om met de gevolgen van lymfoom om te gaan, varieerden. Ze waardeerden de sociale steun die ze ontvingen erg, ofschoon relaties soms verslechterden. Overlevenden maakten naast de behandeling, geregeld ander zware levensgebeurtenissen mee waarvoor verpleegkundigen en andere zorgverleners oog zouden moeten hebben. Overlevenden ervoeren de toegevoegde waarde van het nazorggesprek verschillend. Soms leidde het nazorggesprek tot aanvullende interventies voor nazorg, soms zagen deelnemers het nazorggesprek als een afronding van de behandeling. Ook de behoefte aan verdere nazorggesprekken verschilde. Sommige overlevenden zouden meer nazorggesprekken op prijs stellen, afhankelijk van de klachten die ze hebben. Anderen gaven aan dat ze een proactieve huisarts hebben waar ze terecht kunnen.

De verhalen van de overlevenden van lymfoom laten zien hoe ze omgaan met diverse gevolgen van de kanker en de kankerbehandeling in hun dagelijkse leven. Oog hebben voor steun vanuit het sociale netwerk, de zelfmanagement-vaardigheden en de patiënt als persoon (en niet slechts als patiënt) kan verpleegkundigen en andere zorgverleners ondersteunen bij het aanbieden van nazorg op maat.

Hoofdstuk 8 presenteert de algemene discussie. In dit laatste hoofdstuk wordt allereerst een overzicht gegeven van de belangrijkste resultaten, vervolgens worden diverse methodologische en theoretische overwegingen besproken en tenslotte komen mogelijke implicaties van het onderzoeksproject voor de praktijk, voor onderwijs en voor onderzoek, aan de orde.

We concludeerden dat het belangrijk is voor verpleegkundigen om onderzoek dat samengevat, kritisch beoordeeld en vertaald is in bijvoorbeeld evidence-based 
richtlijnen, te gebruiken binnen evidence-based besluitvorming. Ook is het belangrijk om de patiënt altijd uit te nodigen in de besluitvorming binnen de chronische zorg. We gaven een suggestie hoe EBP en gezamenlijke besluitvorming geïntegreerd kunnen worden en we ontwikkelden een gespreksmodel (waarin EBP en gezamenlijke besluitvorming geïntegreerd zijn) voor nazorg bij kanker. In het gespreksmodel zijn 'Patient-reported outcome measures' (PROMs) en visuele tools, opgenomen. Ook ondersteunt het gespreksmodel het stellen van doelen. Het gebruik van het gespreksmodel ondersteunt mogelijk het aanbieden van nazorg op maat.

Voor de toekomst adviseren we dat, binnen de verpleegkundige beroepspraktijk, de processtappen van EBP worden aangepast aan het opleidingsniveau van verpleegkundigen. Verder adviseren we dat een systematische aanpak wordt gebruikt om de zorg af te stemmen op chronische patiënten. We adviseren voor onderwijs, dat docenten verpleegkunde EBP en gezamenlijke besluitvorming integreren en een gespreksmodel gebruiken, zoals het model dat wij ontwikkeld hebben.

Tijdens ons onderzoek liepen we tegen diverse belemmerende factoren aan ten gevolge van een fusie van twee ziekenhuizen. Daarom adviseren we om zorgvuldig af te wegen of je een onderzoek of implementatie van complexe interventies gaat uitvoeren in een dynamische context, zoals kan ontstaan tijdens een fusie. 
Valorisation

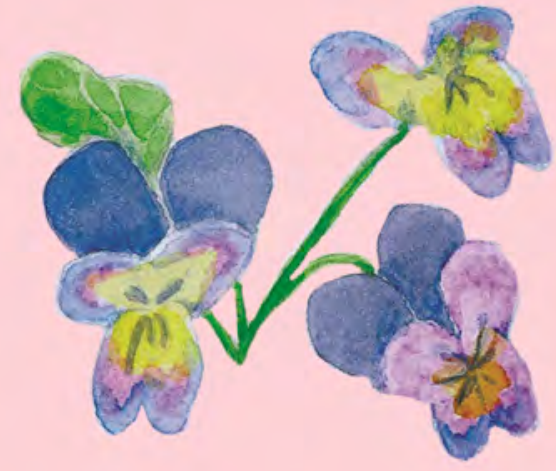





\section{Valorisation}

The aim of this thesis was to gain knowledge about the implementation of evidencebased practice (EBP) in nursing, to find a way to integrate shared decision making (SDM) with EBP in a chronic care environment in nursing, and to develop a strategy for an integrated approach of EBP and SDM in daily nursing practice in the individual aftercare for cancer survivors. The research provided knowledge of teaching and implementation of EBP adapted to the level of education of nurses. Furthermore, the findings provided insights in how to integrate EBP and SDM in chronic care, which resulted in a conversation approach (in which both EBP and SDM are integrated) for use in cancer aftercare. Some tools based on the conversation approach were digitised and integrated in a patient decision aid (PtDA) for breast cancer aftercare and in a general website for patients to prepare for an aftercare consultation. Experiences of lymphoma survivors in early aftercare, after having been exposed to the conversation approach, and perceived barriers and facilitators for health care professionals to adopting the PtDA for breast cancer aftercare have been studied.

Besides the scientific value of the findings of the research project, the findings also have societal value. The aims and findings of this research are relevant for chronic care patients especially cancer survivors, for health care professionals and health care and governmental organizations, and for lecturers and student in health care education. To become valuable, the findings have to be communicated to these stakeholders: to the scientific community, to health care professionals working in cancer care settings or other chronic care settings, to chronic care patients especially cancer survivors, and in educational settings. The process of value creation from knowledge by making it applicable and available for economic and societal utilization, by translating it into products, services and processes, is called 'valorisation'. In this valorisation chapter, the findings of the research project presented in this thesis are discussed with regard to the following aspects: relevance and innovativeness of the findings, translation of the findings to various target groups, and further activities and dissemination.

\section{Relevance and innovativeness}

In this section, the relevance and innovativeness of the research project is described in relation to the integration of EBP and SDM in nursing and to the conversation approach for cancer aftercare. 


\section{The integration of evidence-based practice and shared decision making in nursing}

In the course of this research project, there was a growing opinion in literature that the singular focus on research evidence in EBP should gave way to a more multifaceted focus, in which summarised, critically appraised research evidence is integrated and the values of the individual patient and the patient's context are taken into account. ${ }^{1-3}$ Our research findings contributed to this growing opinion. We pleaded to always invite chronic care patients in decision making, and we suggested and visualized how to integrate EBP and SDM in chronic care in nursing. Suffering from a chronic condition affects a person's life. Chronically ill people may encounter consequences like fatigue, insecurity, depression and restrictions in daily functioning. They are challenged to deal with these consequences and often have to adapt their lives to cope with these consequences. ${ }^{4,5}$ To support these patients in managing their condition in their daily lives, it is important to involve them in decision making and provide them with a personalised approach that addresses their individual experiences, preferences and values. $^{6-10}$

\section{A conversation approach for cancer aftercare}

For many people cancer survival, regardless of their disease status, increasingly means living with a chronic condition ${ }^{11}$ for which a lot of decisions have to be made with regard to evidence based aftercare. ${ }^{12,13}$ In the light of cancer aftercare, it is especially relevant to support nurses with a systematic feasible conversation approach to facilitate shared goal-setting and shared decision-making in daily practice to improve cancer aftercare in line with recommendations of evidence-based practice guidelines. ${ }^{13-16}$

We developed a conversation approach of which the innovativeness lies in an explicit focus on SDM about evidence-based cancer aftercare interventions directed at patient values and goals. Furthermore, the approach for cancer aftercare integrates already used patient-reported outcome measures (PROMs) to support the implementation of PROMs in routine care, which is a topic for improvement in daily health care practice. ${ }^{17,18}$ Another important aspect of the conversation approach is the strong integration of supporting tools derived from currently used and existing tools from evidence-based clinical practice guidelines and the Netherlands Comprehensive Cancer Organization (IKNL). The graphic tool 'four domains of life ${ }^{19-21}$ is based on the framework of the International Classification of Functioning Disability and Health (ICF). ${ }^{22}$ The holistic conversation approach and the four domains of life emphasize the concept of 'positive health", ${ }^{23,24}$ in which health is not merely the absence of disease or disorders, but is defined as the ability to adapt to and to self-manage disorders or disease. The concept of 'positive health' is being increasingly adopted in Dutch health care. 
By digitising and integrating some tools (including the PROMs questionnaire) based on our conversation approach in a PtDA for aftercare in breast cancer ${ }^{25}$ (https://beslissamen.nl/pda_launch.html?pda=tools/pda_borstnazorg_nl/story_html5.ht $\mathrm{ml}$ ) and in a general website for patients to prepare an aftercare consultation (https://eyespirations.com/wpcontent/uploads/ADRZ/Voorbereiding\%20nazorggesprek \%20-\%20Storyline\%20output/story_html5.html) we facilitated the use of these tools for SDM and the embedding of these tools in care processes.

\section{Target groups}

The results reported in this thesis may be relevant for several target groups: chronic patients, especially cancer survivors; nurses and other health care professionals, students and lecturers, health care and governmental organisations, and researchers.

\section{Chronic patients, especially cancer survivors}

To support chronic patients with managing their own condition, it is important to involve them in decision making and to provide them with a personalised approach that addresses their individual values and goals. ${ }^{13-16}$

Moreover, informed patients increasingly have a desire to be involved in decision making. ${ }^{26}$ The developed conversation approach might facilitate cancer survivors to receive tailored aftercare. ${ }^{20,27}$ The PROMs that are incorporated in the conversation approach and the tool that visualizes domains of life, may facilitate patients discussing their actual problems and setting goals after cancer treatment. In the research provided within the thesis we explicitly focused on in depth experiences and stories of patients in cancer aftercare to actually give them a voice.

\section{Nurses and other health care professionals}

The research in this thesis creates awareness of the relevance of integrating EBP and SDM in chronic care and provides nurses and other health care professionals with tools how to accomplish this.

Although research and evidence-based practice guidelines acknowledge that patients should be provided with tailored evidence based cancer aftercare, we learned from analysing practice that nurses and other health care professionals do not always provide patients with this. Our studies revealed that the conversation approach might support nurses to reach more in-depth patient centred conversations and provide patients with more tailored aftercare. ${ }^{20}$ We developed a training and coaching on the job to support nurses with conducting the conversation approach.

The studies revealed important challenges to implement the conversation approach 
such as the experienced difficulties of nurses to elicit patients' preferences and set goals. To actually reach implementation of the approach it is important that the approach is compatible with and embedded in care processes. Moreover, the nurses need to be confident and able to conduct the approach. ${ }^{20,28}$

\section{Students and lecturers}

The results of the research reported in this thesis are relevant to implement in the curricula of nursing science and other health care faculties. The new educational bachelor profile of nurses describes the competencies of nurses according to the 'Canadian Medical Education Directives for Specialists' (CAN meds) roles. ${ }^{29}$ These CAN meds roles are broadly adopted in faculties for health care students. The CAN meds role 'reflective EBP professional' outlines the EBP competencies for future health care professionals and in the CAN meds role 'Collaborator', SDM competencies are outlined. Based on the results of our research, we recommend to start with teaching students to think critically and apply the nursing process steps. Subsequently, to teach nursing students the competencies to conduct the process steps of EBP focused on major care topics and to integrate (Dutch) evidence-based clinical guidelines in the SDM process for authentic patient cases. Then, students can be taught to search summarised, critically appraised evidence, like systematic reviews and other research studies in databases like PubMed. To understand the level of the quality of research evidence they need knowledge about different research designs. We, further, recommend that secondary vocationally or medium level trained nurses are taught the competencies to use EBP guidelines and protocols and to support self-management of patients.

The developed conversation approach with the tools, can be used to actually teach students how to conduct an integrated approach of EBP and SDM into daily practice. We learned that it is important to teach students the theoretical concepts of the approach and to train them in using the conversation approach with authentic patient cases throughout the curriculum.

\section{Health care and governmental organisations}

Our studies were conducted mainly with nurses within the hospital setting. Within hospitals there is growing attention for implementing EBP and SDM on the work floor. Training nurses to become clinical nurse leaders might facilitate implementation of EBP and SDM within an organisation. ${ }^{30-34}$

The results of our research might provide hospitals with insights in how to teach and implement EBP and SDM on the work floor. For nurses, especially secondary vocationally or medium level trained nurses, it is difficult to conduct the process steps of EBP. We 
suggest adapting the EBP process steps to suit the level of education of the nurses. This can be done by formulating Problem - Intervention (PI) questions or Problem Intervention -Control-(PICO) Outcome questions around major care topics like pain and nutrition, and advising a search strategy focussing on (Dutch) clinical practice guidelines and other summarised evidence, such as systematic reviews and critically appraised topics. To teach and implement EBP it seems important to link EBP to the context in which it is implemented for example by choosing authentic patient cases.

In addition, we advise the hospital management to appoint nurse practitioners or other nurses with a master's degree to support the nurses on the ward with the EBP process steps, especially regarding efficiently searching the research literature and critically appraising the evidence.

The basic framework of the conversation approach can be used to teach nurses how to conduct an integrated approach of EBP and SDM in daily practice. The conversation approach is not only relevant for the hospital setting, but for different chronic care settings as well.

Furthermore our research contributes to building bridges across the societal paradigm shift that is reflected in a recent Dutch national report. ${ }^{1}$ It also may help to improve cancer aftercare as advised in a report from the Dutch Health Council. ${ }^{35}$

\section{Researchers}

The results of the studies presented in this thesis contribute to the body of knowledge about implementation of EBP and its integration with SDM in a chronic care environment in nursing, especially in the individual aftercare for cancer survivors.

To build bridges across the paradigm shift of EBP, we demonstrated how to use a variety of study designs with elements of action research that not only fit the aim but also the setting of the studies. Gaining an in-depth insight in experiences of patients, as we did in experiences of lymphoma survivors in early aftercare, also fits within the paradigm shift of EBP that research should focus more on patient experiences. ${ }^{36}$

Researchers might learn from our challenges with practice based research in a dynamic context, as we experienced during the merge of hospitals. Practice based research in a dynamic context requires flexibility of the researchers. It is neccesarry to follow the dynamics of the context to make sure that the main stakeholders are involved and that products are jointly developed and finally implemented in daily practice.

Conducting the research reported on in this thesis contributed to the collaboration between different researchers not only at Zuyd University of Applied Sciences, but also at Maastricht University. We even combined part of the research of this PhD trajectory with two other PhD trajectories, the first in which a similar conversation approach was implemented in family medicine practice and the second in which tools based on our 
conversation approach were digitised and integrated in a patient decision aid for breast cancer aftercare.

Finally, we suggested several topics for further research, like exploring the use of the underlying concept and tools of the conversation approach earlier on in the care trajectory and conducting a large process evaluation.

\section{Activities and further dissemination}

Already early in the project we started to disseminate our results in different ways and on different occasions: in publications and conferences, to health care organisations, to health care faculties, and to lifelong learning. We furthermore started several activities to disseminate the results in near future.

\section{Publications and conferences}

We published several papers in international, peer reviewed journals and in national journals.

The results of the studies have been presented on several national and international conferences by means of posters and oral presentations.

\section{Health care organisations}

Since, 2016, Zuyd University of applied sciences cooperates with Zuyderland Medical Center to teach nurses EBP and SDM competencies, to conduct projects to improve nursing care and to conduct nursing research. Patient discussion meetings based on EBP principles are implemented in daily nursing practice on a growing number of care units. The results of our research are used to successfully teach EBP and SDM competencies and to implement patient case discussion meetings and improve nursing care.

Although, implementation of EBP still is not easy, progress has been made since the start in 2016. On four wards nurses now structurally conduct patient case discussion meetings. On each ward two to three nurses take the lead in these meeting. These nurses learn from each other. To make clinical leadership happen, it is important to professionalise the nursing profession.

Although the conversation approach and PtDA are not broadly implemented in cancer aftercare yet, the NABON guideline for breast cancer refers to the PtDA. ${ }^{37}$

A further important aspect of the conversation approach is that the basic framework can be used for other chronic care settings than cancer aftercare. Lenzen et al. ${ }^{19}$ implemented the approach in Dutch primary care organisations and the approach is recommended for use within Dutch family medicine. ${ }^{38}$ 


\section{Health care faculties}

The results of the studies, have already been partly implemented in the health care faculties of Zuyd University of Applied Sciences. EBP training in the first years of the curricula focus on finding and applying clinical practical guidelines for authentic patient cases. In the final years of training, students learn to use literature found in PubMed and other English databases.

The developed basic conversation approach is embedded within the new curriculum of the bachelor of nursing, within the bachelor occupational therapy and in an interprofessional minor about rehabilitation for nursing-, occupational therapy- and physical therapy students

Students are taught to conduct the approach and practice it with real patients during practical learning.

The conversation approach was studied in the context of several bachelor theses. A group of nursing students further explored the feasibility of the visual tool within family medicine practice, one medical student explored the feasibility and acceptability of the conversation approach for cancer aftercare in family practice, and a group of occupational therapy students used the visual tool to train occupational therapists in goal setting.

\section{Lifelong learning}

SDM and the content of the conversation approach are integrated in a teaching program for lifelong learning for health care professionals working in oncology and in a programme for empowering leadership competencies in community nurses.

\section{Activities to further disseminate the results}

To further implement the conversation approach in oncology care pathways in Zuyderland Medical Center a research proposal to receive research funds, has been submitted. In the near future further activities will be undertaken to implement the results of the studies in health care settings and in curricula of universities.

Recently, the conversation approach and the results of the pretest and the narrative inquiry have been presented by the PhD student of this thesis (JF), the advanced practice nurse of the malignant lymphoma care pathway of Zuyderland Medical Center (DQ) and a patient (JP) at a conference of the Quality of Care consortium of Netherlands Federation of University Medical Centers about Value Based Health Care (https://www.qruxx.com/samen-beslissen-verbetert-zorg-in-voor-en-natraject/). 


\section{Literature}

1. Council for Health and Society of the Netherlands. Zonder context geen bewijs: over de illusie van evidence-based practice in de zorg [No evidence without context. About the illusion of evidence-based practice in healthcare]. The Hague: 2017.

2. Felder M, Meerding WJ. Een toekomst voor Evidence-Based Medicine? [A future for Evidence-Based Medicine?] Achtergrondstudie bij het advies "Zonder context geen bewijs. Over de illusie van evidencebased practice in de zorg." [Background study for the report "No evidence without context. About the illusion of evidence-based practice in healthcare]. The Hague: Council for Health and Society of the Netherlands. 2017.

3. Brives C, Le Marcis F, Sanabria E. What's in a Context? Tenses and Tensions in Evidence-Based Medicine. Med Anthropol. 2016;35(5):369-76.

4. Lawn S, Schoo A. Supporting self-management of chronic health conditions: common approaches. Patient Educ Couns. 2010;80(2):205-11.

5. World Health Organization 2008-20013: action plan for the global strategy for the prevention and control of non-communicable diseases. World Health Organization, 2009. Retrieved from: http://apps.who.int/iris/bitstream/handle/10665/44009/9789241597418_eng.pdf?sequence=1

6. Clark NM, Nelson BW, Valerio MA, Gong ZM, Taylor-Fishwick JC, Fletcher M. Consideration of Shared Decision Making in Nursing: A Review of Clinicians' Perceptions and Interventions. The Open Nursing Journal. 2009;3:65-75.

7. Zoffmann V, Harder I, Kirkevold M. A Person-Centered Communication and Reflection Model: Sharing Decision-Making in Chronic Care. Qual Health Res. 2008;18(5):670-85.

8. Coulter A, Entwistle VA, Eccles A, Ryan S, Shepperd S, Perera R. Personalised care planning for adults with chronic or long-term health conditions. Cochrane Database Syst Rev. 2015(3):Cd010523.

9. Bodenheimer T, Handley MA. Goal-setting for behavior change in primary care: an exploration and status report. Patient Educ Couns. 2009;76(2):174-80.

10. Bodenheimer T, Lorig K, Holman H, Grumbach K. Patient self-management of chronic disease in primary care. JAMA. 2002;288(19):2469-75.

11. Phillips JL, Currow DC. Cancer as a chronic disease. Collegian (Royal College of Nursing, Australia). 2010;17(2):47-50.

12. Cancer research Uk. 2012. Retrieved from: http://www.cancerresearchuk.org/health-professional/ cancer-statistics/survival\#heading-Zero

13. Integraal Kankercentrum Nederland [Comprehensive Cancer Centre the Netherlands ] (IKNL). 2011. Richtlijn Herstel na Kanker [Guideline Cancer survivorship care]. Retrieved from: http://www.oncoline.nl/herstel-na-kanker.

14. Visserman EA, Gijsen BCM, Blaauwbroek HG. 2014. Zorgstandaard Kanker [Care Standard for Cancer]. Leven met Kanker [Living with cancer], IKNL, KWF Kankerbestrijding, Utrecht.

15. Integraal Kankercentrum Nederland [Comprehensive Cancer Centre the Netherlands] (IKNL), 2017. Richtlijn: detecteren behoefte psychosociale zorg [Guideline: Detecting the need for psychosocial care]. Retrieved from: http://www.oncoline.nl/detecteren-behoefte-psychosociale-zorg. (Accessed 24 April 2018).

16. Integraal Kankercentrum Nederland [Comprehensive Cancer Centre the Netherlands] (IKNL), 2017. Richtlijn Medische Specialistische Revalidatie bij Oncologie. [Guideline Medical Specialist Oncological. Rehabilitation] Retrieved from: https://richtlijnendatabase.nl/uploaded/docs/Oncologie/RL_Medisch_ Specialistische_Revalidatie_bij_Oncologie_voor_autorisatie_def.pdf.

17. Howell D, Molloy S, Wilkinson K, Green E, Orchard K, Wang K, et al. Patient-reported outcomes in routine cancer clinical practice: a scoping review of use, impact on health outcomes, and implementation factors. Ann Oncol. 2015;26(9):1846-58.

18. Basch E, Snyder C. Overcoming barriers to integrating patient-reported outcomes in clinical practice and electronic health records. Ann Oncol. 2017;28(10):2332-3.

19. Lenzen SA, Daniels R, van Bokhoven MA, van der Weijden T, Beurskens A. What makes it so difficult for nurses to coach patients in shared decision making? A process evaluation. Int J Nurs Stud. 2017;80:1-11. 
20. Friesen-Storms JHHM, Bours GJJW, Snijders ICG, van der Weijden T, Jie K-SG, Beurskens AJHM. A conversation approach based on shared goal-setting and shared decision-making for nurses in cancer aftercare: A developmental study. Eur J Oncol Nurs. 2018;35:107-16.

21. Lenzen SA., Daniels R, van Bokhoven MA, van der Weijden T, Beurskens A. Development of a conversation approach for practice nurses aimed at making shared decisions on goals and action plans with primary care patients. BMC Health Services Research. 2018; 18 (891). https://doi.org/10.1186/s12913-018-3734-1

22. World Health Organisation. International Classification of Functioning, Disability and Health (ICF). 2001

23. Huber M, van Vliet M, Giezenberg M, Winkens B, Heerkens $Y$, Dagnelie PC, et al. Towards a 'patientcentred' operationalisation of the new dynamic concept of health: a mixed methods study. BMJ Open. 2016;6(1):e010091.

24. Huber M, Knottnerus JA, Green L, Horst Hvd, Jadad AR, Kromhout D, et al. How should we define health? BMJ. 2011;343:d4163.

25. Klaassen LA, Dirksen CD, Boersma LJ, Hoving C, Portz MJG, Mertens PMG, et al. A novel patient decision aid for aftercare in breast cancer patients: A promising tool to reduce costs by individualizing aftercare. The Breast. 2018;41:144-50.

26. Politi MC, Dizon DS, Frosch DL, Kuzemchak MD, Stiggelbout AM. Importance of clarifying patients' desired role in shared decision making to match their level of engagement with their preferences. Bmj. 2013;347:f7066.

27. Friesen -Storms, JHHM, Bours G, Quadvlieg-Delnoy D, Moser A, Heijmans J, van der Weijden T, Beurskens AJHM, Jie KSG. There is more to learn from stories of lymphoma survivor's to further improve aftercare planning: A narrative inquiry. Submitted August 2018.

28. Klaassen L, Friesen S, J.H.H.M., Bours GJJW, Dirksen C, Boersma L, Hoving C. Perceived Facilitators and Barriers for Healthcare Professionals to Adopting a Patient Decision Aid for Choosing Breast Cancer Aftercare: a cross-sectional study. Submitted November 2018.

29. Stuurgroep bachelor of nursing 2020 [Steering group bachelor of nursing 2020]. Bachelor Nursing 2020: Een toekomstbestendig opleidingsprofiel 4.0 [Bachelor of nursing 2020: A future proof educational profile]. Landellijk Overleg Opleiding Verpleegkundigen, 2015.

30. Ott KM, Haddock KS, Fox SE, Shinn JK, Walters SE, Hardin JW, et al. The Clinical Nurse Leader: impact on practice outcomes in the Veterans Health Administration. Nursing economic\$. 2009;27(6):363-70, 83; quiz 71.

31. Jukkala A, Greenwood R, Motes T, Block V. Creating innovative clinical nurse leader practicum experiences through academic and practice partnerships. Nursing Educ Perspect. 2013;34(3):186-91.

32. Garner BL, Metcalfe SE, Hallyburton A. International collaboration: A concept model to engage nursing leaders and promote global nursing education partnerships. Nurse Educ Pract. 2009;9(2):102-8.

33. Saunders H, Vehvilainen-Julkunen K. Nurses' Evidence-Based Practice Beliefs and the Role of EvidenceBased Practice Mentors at University Hospitals in Finland. Worldviews Evid Based Nurs. 2017;14(1):3545.

34. Stetler CB, Ritchie JA, Rycroft-Malone J, Charns MP. Leadership for evidence-based practice: strategic and functional behaviors for institutionalizing EBP. Worldviews Evid Based Nurs. 2014;11(4):219-26.

35. The Health Council of the Netherlands, 2007 Follow-up in oncology. Identify objectives, substantiate actions. The Hague: Health Council of the Netherlands; 2007. Retrieved from: https://www.gezondheidsraad.nl/en/task-and-procedure/areas-of-activity/optimum-healthcare/followup-in-oncology-identify-objectives.

36. Greenhalgh T, Howick J, Maskrey N. Evidence based medicine: a movement in crisis? Bmj. 2014;348:g3725.

37. Integraal kankercentrum Nederland [Comprehensive Cancer Centre the Netherlands] (IKNL). NABON Richtlijn Mammacarcinoom [NABON Guideline Mammacarcinoma]. Revised 2017. Retrieved from: https://www.oncoline.nl/index.php

38. Nederlands Huisartsen Genootschap (NHG) [The Dutch College of General Practitioners ] Gezamenlijke besluitvorming [Shared Decision Making] 2017. Retrieved from: https://www.nhg.org/sites/default/ files/content/nhg_org/uploads/gezamenlijke_besluitvorming_a4_kaart_sk_2017_Ir.pdf 
Dankwoord

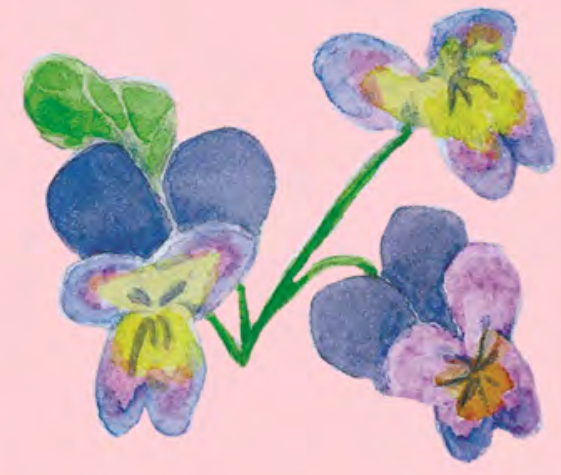





\section{Dankwoord}

Hier ligt dan mijn proefschrift. Het werken aan dit proefschrift vormde jarenlang een rode draad in mijn leven. Tijdens die jaren heb ik veel steun en hulp ervaren van mijn promotieteam, maar ook van vele andere mensen. In mijn dankwoord wil ik een speciaal woord richten tot al die mensen die geholpen hebben om dit proefschrift tot een goed einde te brengen.

Allereerst wil ik mijn promotieteam bedanken, Gerrie Bours, Sandra Beurskens, Trudy van der Weijden en Asiong Jie. Promoveren doe je niet in je eentje en zonder jullie begeleiding als team was het me niet gelukt om dit proefschrift tot een goed einde te brengen. Er was eenheid in verscheidenheid en dat maakte ons als team juist sterk. Toen mijn promotieonderzoek door de fusie van Zuyderland Medisch Centrum in moeilijker vaarwater kwam, bleven jullie mij stimuleren om andere wegen te zoeken, ondanks dat jullie volgens mij zelf ook zorgen hadden over de voortgang van het traject. Uiteindelijk heeft dat volhouden zich geloond!

Gerrie, dankzij een lunch met jou ben ik ooit betrokken geraakt bij het lectoraat Autonomie en Participatie van Chronisch Zieken. We raakten aan de praat over onze achtergrond en de week erna belde je thuis bij ons op of ik mee wilde doen aan een evaluatieonderzoek naar de implementatie van de richtlijn: 'Revalidatie na een beroerte'. Na enige aarzeling heb ik dit met beide handen aangegrepen en hiervan heb ik geen spijt gehad. Na dit onderzoek vroeg je of ik interesse had in een promotieonderzoek, maar hieraan was ik nog niet toe. Ik was de gelukkige moeder van drie prachtige kinderen en had het verlangen naar nog een vierde kind. Dit leek me op dat moment niet verenigbaar met promoveren. Toen onze vierde richting de leeftijd kwam dat hij naar de basisschool ging, wilde ik het promoveren heroverwegen. Door diverse onderzoeksprojecten waarin ik geparticipeerd had in die tijd, was mijn passie voor onderzoek gegroeid. Toen kreeg ik, mede dankzij jou, de kans om projectleider te worden van een onderzoek binnen het voormalige AtriumMC met als doel het implementeren van EBP binnen de verpleegkundige beroepsgroep. Dit was de eerste opstap naar mijn promotieonderzoek. Gerrie, dank voor de potentie die jij in die tijd in mij zag en het vertrouwen dat je me in de afgelopen jaren hebt geschonken. Je liet me mijn gang gaan en was er als ik je nodig had. Je reageerde altijd snel op mijn mails en ik mocht je storen, ook als dat misschien minder goed uitkwam voor jou. Ik kon altijd met vragen bij jou terecht. Binnen het promotieteam had jij vaak net een suggestie of een idee dat ikzelf of het team nog niet gezien had en dat mij weer verder hielp. Je gaf altijd goede kritische feedback op mijn artikelen, waardoor de kwaliteit ervan toenam. 
Ook konden we privé omstandigheden met elkaar delen en dat is in een dergelijk intensief traject belangrijk.

$\mathrm{Nu}$ ben ik betrokken bij een scholingstraject binnen Envida, waarvan jij de projectleider bent. Ik hoop dat we in de toekomst nog vaker samen kunnen werken. Gerrie, bedankt voor alles in de afgelopen jaren.

Sandra, heel erg bedankt dat jij in mij de potentie zag om aan een promotieonderzoek te beginnen. Je gaf mij het gevoel dat jij mij in jouw team wilde. Toen een andere lector mij na de bevalling van onze vierde, Timo, belde of ik wilde gaan promoveren op een promotieonderzoek binnen diens lectoraat, liet jij mij duidelijk blijken dat jij mij niet kwijt wilde en dat ik naar jou toe kon komen als ik eraan toe was. Dit was een aantal jaren later het geval en toen gaf jij mij die kans. Je dacht ook mee hoe dit te realiseren was met een gezin met vier kinderen. Sandra, ik heb veel gehad aan onze brainstormsessies, zowel binnen het teamoverleg als buiten het teamoverleg. Dat hielp mij weer om verder de lijn door te zetten en te structureren. Jij hebt een tomeloze energie, die aanstekelijk werkt. Ook jij gaf altijd goede kritische feedback op artikelen. Daarnaast heb ik ook door jou geleerd om in figuren, schema's en beelden te denken en deze ook op te nemen in mijn artikelen om de lezers van het artikel te ondersteunen. Jij hebt me verder gestimuleerd om assertiever te zijn en te staan voor mijn mening. Ik ben daarin door mijn promotieonderzoek heel erg gegroeid. Sandra, ook heel erg bedankt dat je me de ruimte gaf toen het niet goed ging met mijn moeder om er voor haar te zijn. Jij zei toen: "Neem de tijd. Dit kun je nooit meer overdoen". Daar ben ik jou en ook de rest van het team heel dankbaar voor. Sandra, bedankt voor alles in de afgelopen jaren.

Trudy, ik weet nog dat jij op onze eerste afspraak zei: "Promoveren met vier kinderen, realiseer je dat het geen 9.00-17.00 baan is". En dat is het inderdaad ook niet. Toen we er samen voor gingen, gaf jij mij steeds het vertrouwen dat het wel zou lukken. Jij straalt altijd rust en wijsheid uit en je had in team overleggen vaak een helikopter view. Bij jou denk ik aan eenvoud in deskundigheid. Je weet heel erg veel en hebt op onderzoeksgebied al veel bereikt, maar je blijft gewoon wie je bent. Je hebt een heel persoonlijke 'touch'. Ook heb je heel veel oog voor de mens achter de promovendus. Trudy, bedankt voor alles in de afgelopen jaren.

Asiong, fijn om ook een man in het team erbij te hebben. Jij verrijkte het promotieteam vanaf 2013. Je bent een arts met een warm hart voor zijn patiënten. Jij maakt je sterk voor geïntegreerde zorg, waarbij zowel aandacht is voor cure als voor care, die afgestemd is op de individuele patiënt. Wij konden jouw kennis over de oncologie heel goed gebruiken in ons team. Ook gaf je mij het vertrouwen om een voorstel voor een 
Zuyderland subsidie samen met jou te schrijven. Ik hoop dat we in de toekomst nog veel subsidieaanvragen samen mogen schrijven en dan natuurlijk ook de projecten mogen uitvoeren. Asiong, bedankt voor alles in de afgelopen jaren.

Naast mijn promotieteam wil ik ook andere mensen bedanken die een bijdrage hebben geleverd aan het proefschrift. In de eerste plaats wil ik alle patiënten en zorgverleners bedanken die deel hebben genomen aan de studies in mijn proefschrift. Een aantal mensen hebben deelgenomen als ervaringsdeskundige experts in dit proefschrift: meneer R. en meneer B., bedankt voor het meedenken over het vorm geven aan het nazorggesprek. Mevrouw B., dank voor uw medewerking aan de film over het nazorggesprek. Meneer P., dank voor het meedoen met een van de symposia en de bereidheid om deel te nemen aan de projectgroep van het nieuwe onderzoeksvoorstel voor Zuyderland.

Ook gaat veel dank uit naar een aantal kartrekkers van mijn project binnen Zuyderland: Danny Quadvlieg-Delnoy, Ingrid Snijders, Astrid Robroek-Schaeken, Edith Claessens en Christel Dacier-Herfs. Jullie hebben mee vorm gegeven aan het nazorggesprek en zijn er enthousiast in de praktijk mee aan de slag gegaan. Mede door jullie heb ik het onderzoek naar het nazorggesprek vorm kunnen geven. Mijn dank hiervoor! Danny, fijn dat jij in het kader van je eigen opleiding tot verpleegkundig specialist verder onderzoek bent gaan doen naar het nazorggesprek.

Susanne Verboort-Bogers, dank voor jouw adviserende rol ten aanzien van nazorg bij kanker vanuit het Integraal Kankercentrum Nederland.

Ook veel dank aan de mensen die bijgedragen hebben aan het implementeren van EBP binnen het voormalige AtriumMC, de verpleegkundigen van de longafdeling Atrium met name Henriëtte Erkens en Imane Daher. De verpleegkundigen van de afdeling neurologie met name George Penders, Indra Renkens en Sanne van Haandel. De verpleegkundigen van de Verpleegkundige Adviesraad met name Annemiek Klaassen, Ada Roozeboom, Annamaria Hazen-Verlaan, Marieta Brand en Charlotte Boermans. Paul van der Putten en de praktijkbegeleiders met name Jean Schuivens en Nick Olfen van het leerhuis van het voormalige AtriumMC nu Zorgacademie Zuyderland. En alle mensen die hebben bijgedragen en die ik niet met naam genoemd heb.

Een speciaal woord van dank gaat ook zeker uit naar Linda Klaassen en Liesbeth Boersma.

Jullie vertrouwen om met mij samen te werken en de krachten van onze beider promotietrajecten te bundelen, heeft uiteindelijk geresulteerd in een mooi resultaat. Jullie waren niet alleen hele fijne samenwerkingspartners, maar we konden ook 
persoonlijke dingen met elkaar delen. Dank voor de hele fijne samenwerking en ik hoop dat we in de toekomst nog vaker onze krachten kunnen bundelen.

Ciska Hoving en Carmen Dirksen, jullie zijn onderdeel van het promotieteam van Linda en ook aan jullie mijn dank voor de samenwerking in het project.

Dan rest me nu nog een woord van dank aan al mijn collega's van zowel het lectoraat Autonomie en Participatie van Chronisch Zieken als van de HBO-V, van leven lang leren, van de master ANP en van andere opleidingen van de faculteit gezondheidszorg van Zuyd Hogeschool. Daarnaast natuurlijk ook dank aan alle collega's van de vakgroep huisartsgeneeskunde, de vakgroep Health Services Research en het Care and Public Health Research Institute (CAPHRI) van de Universiteit Maastricht. Ik kan ze niet allemaal opnoemen, want dan wordt dit dankwoord veel te omvangrijk en loop ik het risico iemand te vergeten.

Maar door jullie continue interesse voor mijn proefschrift, mijn vorderingen en bemoedigende woorden hebben jullie mij gesterkt om door te gaan.

Een aantal collega's wil ik toch met naam noemen.

Stephie, jij bent mijn paranimf en daarmee ben ik heel blij. We hebben samen een vergelijkbaar gespreksmodel gebruikt, jij binnen de eerste lijn en ik binnen de nazorg voor kanker. Jij was al eerder bezig met de ontwikkeling van het model dan ik en ik heb dankbaar gebruik gemaakt van het vier bollen model dat binnen jouw promotieonderzoek ontwikkeld is. Maar naast de samenwerking in de projecten, ben je ook een hele fijne, warme en lieve collega. We hebben veel lief en leed samen kunnen delen. Stephie, dank voor alles en heel fijn dat je mijn paranimf wilt zijn. Ik hoop dat we in de toekomst veel met elkaar kunnen blijven delen.

Steffy en Linda, we delen samen de promovendikamer vanaf het begin. Er was altijd belangstelling voor elkaars projecten en ook voor bijzonderheden in onze levens. Vaak staken we elkaar een hart onder de riem. Fijn dat we dit met elkaar konden delen! Dank hiervoor!

Jerome, Ruth, Darcy, Li-Juan, Iris, Hester, Marcel, Renée, Kyra, Kim en Ezra, we hebben voor kortere of langere tijd de promovendikamer gedeeld. Ook mijn dank aan jullie dat we ervaringen, inhoud en gezelligheid konden delen. Dank allemaal voor alles!

Petra, ooit was ik jouw docent op de HBO-V en ik had er veel vertrouwen in dat jij het goed zou gaan doen. Daarin had ik me niet vergist! Jij ging door met de dossierbesprekingen in het Zuyderland en dat doe je zorgvuldig, goed en met veel verve. Jij 
hebt me geraakt toen je zei dat ik ooit een voorbeeldfunctie voor jou vervuld heb op de HBO-V, dat zie ik als een van de belangrijke onderdelen van het docent zijn. Super fijn dat we nu geregeld samenwerken en ik hoop dat we dat mogen blijven doen.

Albine, jij bent de onderzoeker die het project van het eerste artikel van dit proefschrift uitgevoerd hebt en jij hebt een hele grote bijdrage geleverd als medeauteur van dit artikel. Het siert jou enorm dat jij mij de kans gunde om eerste auteur van dit artikel te worden. We zijn samen gaat uit eten om te vieren dat dit artikel zou worden gepubliceerd. Ook steunde je mij als ik even vast zat met het proefschrift. Jij stak me dan een hard onder de riem. Ik was heel blij dat ik jou als expert kon raadplegen met betrekking tot kwalitatief onderzoek. Want daarover weet jij heel veel. Dank voor alles Albine.

Jessie, heel erg bedankt voor de samenwerking tijdens het project op maat implementeren van evidence in de praktijk, waarvan het implementeren van EBP binnen het AtriumMC deel uitmaakte.

Tineke, heel erg bedankt voor de projecten die we samen voor het lectoraat Autonomie en Participatie voor chronisch zieken hebben uitgevoerd en natuurlijk ook voor alle andere werkzaamheden.

Stephanie, Prisca, Marja en Jacqueline dank ook voor al jullie ondersteuning met mijn promotieonderzoek.

Verder wil ik ook een aantal andere mensen bedanken die bijgedragen hebben aan dit proefschrift.

Alle werkstudenten bedankt voor het transcriberen van interviews of mee vorm geven aan de layout van vragenlijsten en figuren.

José Heijmans, dank dat jij samen met mij de interviews voor de narrative inquiry hebt geanalyseerd en mede-auteur bent van het artikel. Jij was hierin heel zorgvuldig.

Tiny Wouters, dank voor de mooie lay-out van mijn proefschrift.

Evelien Jagtman, bedankt voor de prachtige kaft.

Kristine van Asselt en Marieke Hageman, dank dat jullie bereid waren om een afstudeerproject uit te voeren met het gespreksmodel in de eerste lijn. 
De beoordelingscommissie en leden van de corona, bedankt voor het beoordelen van het proefschrift en jullie bereidheid om te opponeren.

De collega's die opponeren bij mijn proefpromotie en waarbij sommigen zelfs het teamuitje van verpleegkunde hebben laten schieten. Bedankt.

Ook hebben mijn familie en vrienden een grote bijdrage geleverd aan dit proefschrift.

Ron heel veel dank dat jij me als echtgenoot de ruimte gaf om te gaan promoveren met een gezin met vier opgroeiende kinderen. Jij vond promoveren wel echt iets voor mij, omdat ik iemand ben die graag diepgaand onderwerpen wil onderzoeken. Ook ben ik altijd wel aan het leren of met een grotere of kleinere studie bezig. Je hebt me altijd onvoorwaardelijk gesteund en als ik het even niet meer zag zitten dan relativeerde jij alles weer even en gaf me de moed om door te gaan. Naast de emotionele steun was jij naast je drukke fulltime baan gewoon ook vaak de huisman. Je nam taken van mij over als ik door mijn concentratie op een artikel of stuk dat ik moest schrijven de tijd vergat. We delen al bijna 23 jaar lief en leed en ik hoop dat we nog heel veel mooie jaren samen hebben.

Robin, Milan, Levi en Timo, als mensen mij vroegen wat ik wilde worden dan zei ik al van jongs af aan: moeder. En dat is gelukt. Oh wat ben ik blij met jullie en wat ben ik trots op de mooie personen die jullie zijn geworden. Ook jullie bedankt voor jullie begrip als mama het eten liet aanbranden omdat ze de tijd vergat als ze geconcentreerd bezig was. Ook kon ik door jullie altijd relativeren dat het leven uit belangrijkere dingen bestaat dan promoveren. Ik hoop dat we altijd zo'n fijne en warme band blijven houden, als we nu hebben en dat we als gezin nog veel samen mogen delen.

Robin ook heel fijn dat jij mijn paranimf bent.

Mam en pap, helaas zijn jullie er allebei niet meer. Papa jij bent al op 11 september 2009 geveld door 'Parki', zoals je dat zelf altijd zei. Ik herinner me jou altijd als een hele lieve en goedlachse man, die kattenkwaad met mij uithaalde als mama er niet was. De laatste jaren werd jouw kwaliteit van leven steeds negatiever beïnvloed door 'Parki'. Je haalde echter steeds veel vreugde uit jouw kleinkinderen. Mama, jij hebt nog meegekregen dat ik begon aan het promotieonderzoek. Je zei niet zo vaak dat je trots op ons was, maar dat hoorden we dan wel terug van andere mensen. Je werd ziek toen ik nog geen jaar bezig was met mijn promotieonderzoek. Ik heb je nog de kleren laten zien die ik aandeed naar de uitreiking van de promotiebeurs voor leraren van de Nederlandse Raad voor Wetenschappelijk Onderzoek (NWO). Ook ben ik heel dankbaar dat ik de laatste zes weken bij jou thuis heb mogen doorbrengen en voor jou heb mogen zorgen samen met 
Chantal, tot je overleed op 10 mei 2013. We waren in die laatste weken echt een eenheid en heel erg op elkaar afgestemd. Zo kon ik ook iets terug doen voor alles dat je voor ons en voor papa hebt gedaan. Mam en pap, dankzij jullie warme en liefdevolle opvoeding ben ik geworden wie ik ben. Ook pasten jullie altijd met veel plezier op de kleinkinderen, jullie waren een geweldige oma en opa.

Aan mijn tantes, de zussen van mam en Theo en Els. Bedankt voor jullie warme inzet tijdens de ziekte van mam.

Chantal en Michel, Chantal samen groeiden wij op als zusjes binnen ons hechte gezin en deelden wij veel lief en leed. Ik was als vierjarige heel blij dat ik een zusje kreeg. Dat wilde ik echt heel graag. Ik had inmiddels met Annie, onze tante en buurvrouw, die negen maanden eerder Imca had gekregen, afgesproken dat mam en Annie zouden ruilen als jij een jongen zou zijn. Gelukkig was dat niet nodig! Chantal, jij ook dank voor je liefde, steun en begrip voor mijn promotieonderzoek. Michel, dank voor de liefde die je met mijn zusje en ons gezin deelt.

Mam en pap Friesen (opa poes), dank ervoor dat jullie door jullie opvoeding Ron mede hebben gevormd tot de persoon die hij is. Mam Friesen, ik heb jou maar kort (circa 1 jaar) maar wel intensief mogen leren kennen. Daarvoor ben ik heel dankbaar. Ron zorgde toen ik jou leerde kennen al vier jaar voor jou omdat je bedlegerig was geworden door uitzaaiingen van kanker. Ik ben heel blij dat ik ook dit deel van het leven van Ron heb mogen ervaren. Opa poes, jij overleed net voordat ik begon met mijn promotieonderzoek. Jij was een hele evenwichtige, rustige man die bijna altijd zijn kalmte behield. Daaraan heb ik vaak gedacht als ik mijn kalmte even niet kon bewaren.

Roel, Astrid, Jamie, Kevin, Davy, Finn en Lloyd, dank voor de afleiding en gezelligheid die jullie boden tijdens mijn promotietijd. Bedankt dat jullie, ondanks jullie eigen zorgen het afgelopen jaar, toch ook aandacht hadden voor mijn promotieonderzoek. We hopen nog veel samen te kunnen delen.

Carine en Megan. Carine, we zijn al 'best friends forever' sinds ons twaalfde jaar en hebben lief en leed met elkaar gedeeld. We hadden elkaar meteen gevonden toen we op onze middelbare school op introductie gingen en we hebben elkaar daarna nooit meer los gelaten. Jij hebt me gesteund om aan dit promotietraject te beginnen, omdat je weet hoe belangrijk goede (na)zorg is. Je ging enthousiast mee naar de uitreiking van de promotiebeurs voor leraren in Den Haag. We plannen geregeld uitstapjes met of zonder kinderen die zorgen voor leuke en gezellige afleiding. Ook jij zorgde ervoor dat ik het 
belang van en de zorgen rondom promoveren geregeld kon relativeren. Carine, je bent heel goed in het schrijven van gedichten. Dat is echt een groot talent van jou. Dank voor je prachtige gedicht voor dit proefschrift. Megan, wat ben ik blij dat je mijn petekind bent. Je bent een prachtige meid. Blijf vooral wie je bent. Carine en Megan, ik hoop dat we nog vele jaren samen lief en leed mogen delen. Bedankt voor de afgelopen jaren.

Paulijn, Guido, Oliver en Ruby, dank voor alles dat wij in onze levens en in de vriendschappen tussen onze kinderen en ons hebben mogen delen. We kwamen in enerverende tijden in elkaar levens en dat heeft onze vriendschap sterk gemaakt. Paulijn, dank voor de gezellige uitstapjes en je luisterend oor. Paulijn, ook dank voor de eerste ontwerpen van het design van mijn proefschriftkaft en de vele mooie foto's die je daarvoor hebt gemaakt. Guido, dank voor het vertalen van de quotes van een van mijn artikelen. Ik hoop dat we nog lang onze levens mogen delen.

Angelique en Jacqueline, de 'Sjilvendse Maedjes', dank dat jullie altijd in mij geloofd hebben. Ook dank voor de gezellige uitstapjes die we als vriendinnen hebben. Ook al is het promoveren voor jullie iets abstracts, jullie zijn er altijd in geïnteresseerd. Ik hoop dat we nog lang 'Sjilvendse Maedjes' mogen blijven.

Lindy, dank voor jouw vriendschap en vaak wijze woorden op het schoolplein en tijdens uitstapjes. Ook heel erg dank dat je kritisch naar de layout en de Nederlandse samenvatting van mijn proefschrift hebt gekeken. Ik hoop dat jij met Bas, Darja en Paulina in ons leven blijft.

Andrea, dank voor je vriendschap en warmte en voor je aanhoudende interesse in waarmee ik bezig ben. Je bent samen sterk met Mark en Milo en Mickey. Ik hoop dat onze jongens net als wij bevriend mogen blijven.

Iris en Yvette, onze vriendschap gaat al terug tot onze studententijd. We hebben veel lief en leed gedeeld. Ik ben altijd blij als we elkaar weer een keer kunnen zien. Ook jullie dank dat jullie altijd in mij zijn blijven geloven en ik hoop dat we nog lang vriendinnen blijven.

Mattie, dank voor de belangrijke mijlpalen die je tijdens ons leven met ons hebt gedeeld en nog deelt. Zeker in de enerverende tijd met mam, was je er altijd als we je nodig hadden. Ik kan met jou goed reflecteren op de zin van het leven. Dank ook voor jouw interesse en steun tijdens mijn promotieonderzoek. 
Elsi, Toos en Mia, ook jullie dank voor jullie vriendschap en interesse in mijn leven en mijn promotieonderzoek.

Ome Jo, ik vind de bezoekjes die je geregeld op vrijdag brengt altijd heel gezellig. Jij en tante Diena hebben als buren van mijn ouders altijd een belangrijke rol in mijn leven gespeeld. Dank voor al jullie warmte, aandacht en interesse.

Tenslotte wil ik iedereen bedanken die ik hier misschien niet heb genoemd, maar die ook op een of andere manier een rol hebben gespeeld in het voltooien van dit proefschrift.

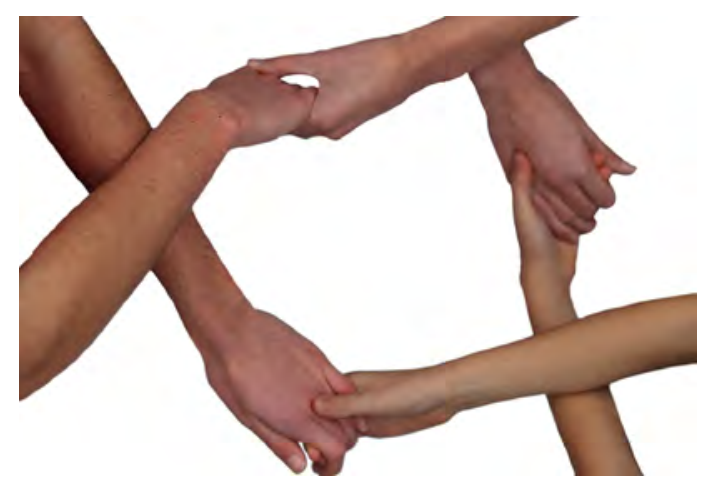

\section{Bergen verzetten}

Met de schoonheid die je ziet,

Met de geestdrift die je wekt

Met de ruimte die je laat,

Met de vrede die je brengt,

Met gevoel voor humor,

Met de zorg voor het geringe,

Met de troost van aandacht,

Met de kracht van eenvoud

Ben je in staat

om bergen te verzetten

(K. Gelaude) 

Curriculum vitae

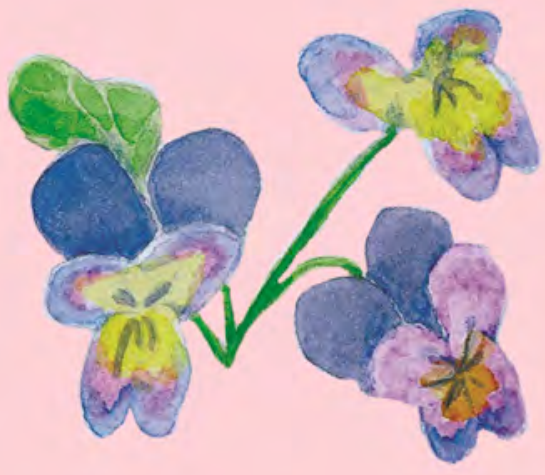





\section{Curriculum vitae}

Jolanda Friesen-Storms, born on 4 May 1970 in Heerlen, lived during her childhood in Schinveld in the Netherlands with her parents and her younger sister Chantal.

In 1988, she graduated from secondary school, Gymnasium Serviam, Sittard. She studied Health Sciences with specialization Biological Health Science at Maastricht University and she graduated as a master of science (doctorandus) in 1992. She then studied at the bachelor of nursing at Hogeschool Sittard (now Zuyd University of Applied Sciences) and graduated in 1994. She first worked as a nurse in a rehabilitation centre in Aachen,

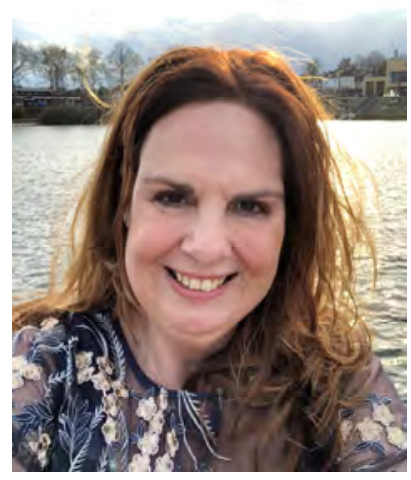
Germany and subsequently she worked as a care coordinator in a home care organization. In 1999 she started working as a lecturer at Zuyd University at the bachelor of nursing. At the same time she studied a second specialization of Health Sciences; Science of Care, from which she graduated in 2003. In 2005 she started working for the Research Centre for Autonomy and Participation of Persons with a Chronic Illness, where she was part of a research team that evaluated the implementation of the Dutch evidence-based guideline of rehabilitation after a stroke. After this study she participated in a study on the development of a measurement instrument for a 'new way of working' in a hospital in the South of the Netherlands and two studies on the implementation of patient centred care in rehabilitation for patients with chronic pain and chronic obstructive pulmonary disease. Jolanda started this PhD project in September 2011. The project was a close collaboration between the Research Centre Autonomy and Participation of Persons with a Chronic IIIness and the Care and Public Health Research Institute (CAPHRI) of Maastricht University. In June 2013 she received a doctoral grant for teachers from the Netherlands Organisation for Scientific Research (NWO). The PhD project resulted in this thesis. During her PhD project Jolanda continued being a lecturer. Currently, Jolanda's work involves a combination of lecturing at the bachelor of nursing, the master of advanced nursing practice and some programs of lifelong learning, and lecturing and research activities for the Research Centre for Autonomy and Participation of Persons with a Chronic Illness. Her activities for the research centre focus on evidence-based practice, shared decision making, implementation of patient reported outcome measures in daily nursing practice and cancer aftercare.

In her private life she married Ron in 1996 and gave birth to their four children: Robin (1999), Milan (2002), Levi (2004) and Timo (2007). 
List of publications

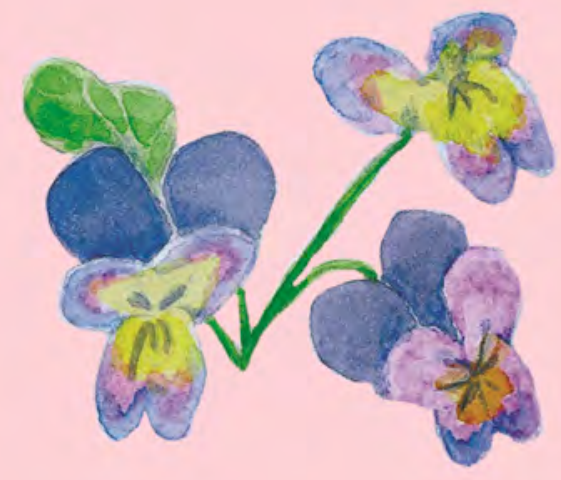





\section{List of publications}

\section{International peer-reviewed publications}

Klaassen LA, Friesen-Storms JHHM, Bours GJJW, Dirksen CD, Boersma LJ, Hoving C. Perceived facilitating and restricting factors for Healthcare Professionals to Adopting a Patient Decision Aid for Breast Cancer Aftercare: a cross-sectional study. Under review: minor revisions. Patient Education and Counseling.

Friesen-Storms JHHM, Bours GJJW, Quadvlieg-Delnoy DJL, Moser A, Heijmans JMJ, van der Weijden T, Beurskens AJHM, Jie KSG. There is more to learn from stories of lymphoma survivor's to further improve aftercare: a narrative inquiry. Submitted.

Friesen-Storms JHHM, Bours GJJW, van der Weijden T, Jie KSG, Snijders ICG, Beurskens AJHM. A conversation approach based on shared goal setting and shared decision making for nurses in cancer aftercare. Eur J Oncol Nurs. 2018;35:107-116.

Friesen-Storms JHHM, Bours GJJW, van der Weijden T, Jie KSG, Snijders ICG, Beurskens AJHM. A conversation approach based on shared goal-setting and shared decision-making for nurses in cancer aftercare: A developmental study. ASCO annual meeting, Chicago. June 2018. Published online: J Clin Oncol 36, 2018 (suppl; abstr e18878).

Friesen-Storms JHHM, Beurskens AJHM, Bours GJJW. How to implement EBP on a hospital unit with mainly medium level educated nurses? Lessons learned. J Cont Educ Nurs. 2017;48(9):407-412.

Friesen-Storms JHHM, Bours GJJW, van der Weijden T, Beurskens AJHM. Shared decision making in chronic care in the context of evidence based practice in nursing. Int J Nurs Stud. 2015;52:393-402.

Friesen-Storms JHHM, Moser A, van der Loo S, Beurskens AJHM, Bours GJJW. Systematic implementation of evidence-based practice (EBP) in a clinical nursing setting: a participatory action research project. J Clin Nurs. 2014;24:57-68. 


\section{National publications}

Friesen-Storms J, Bours G, van der Weijden T, Beurskens S. Het gebruik van shared decision making binnen evidence-based practice: Samen beslissen binnen de verpleegkunde. Nederlands Tijdschrift voor Evidence Based Practice. 2014;9(1):7-9.

Klaassen A, Hazen-Verlaan A, Roozeboom A, Boermans C, Bours G, Friesen-Storms J. Meetinstrument 'Verpleegkundige Standaard':Een afdelingsspecifieke zelfcheck voor verpleegkundige kwaliteit. Tijdschrift voor verpleegkundigen. 2012;122(6):56-60.

Moser A, Friesen-Storms J, Bours GJJW. Implementatie van evidence based practice: belemmerende en bevorderende factoren. Onderwijs en gezondheidszorg. 2012;36(1): 7-9.

Friesen JHHM, Schoot T, Balk S, Beurskens S, Köke A. Cliëntgecentreerde zorg binnen het multidisciplinaire pijnteam van Adelante: naar een permanente plaats op de agenda. Nederlands Tijdschrift voor Revalidatiegeneeskunde. 2012;1:12-16.

Schoot T, Friesen J, Beurskens S. Meten op maat: Ontwikkeling meetinstrument implementatie voor nieuw ziekenhuisbeleid. Onderwijs en gezondheidszorg. 2008;32(7): 19-22.

\section{Oral presentations}

Friesen-Storms JHHM, Quadvlieg-Delnoy DJL, Peters J. Patiëntuitkomsten verbeteren door samen beslissen in de nazorg bij kanker en specifiek bij maligne lymfoom. Symposium Nederlandse Federatie van UMC's consortium kwaliteit van zorg, Nieuwegein. 21 maart 2019.

Friesen-Storms JHHM, Bours GJJW, van der Weijden T, Jie KSG, Snijders ICG, Beurskens AJHM. A conversation approach based on shared goal setting and shared decision making for nurses in cancer aftercare. 1 st International Conference of the German Society of Nursing Science, Berlin, Germany. 4 May 2018.

Quadvlieg-Delnoy D, Friesen-Storms J, Bours G, van der Weijden T, Beurskens A, Jie A. Nazorggesprekken binnen het zorgpad lymfoom: een onderzoeksproject. $8 \mathrm{e}$ Wetenschappelijke Symposium ZuyderlandMC, Sittard. 29 Juni 2017. 
Friesen-Storms J, Bours G, van der Weijden T, Jie A, Beurskens A. Nazorg bij kanker. Onderdeel van een workshop over oncologische revalitatie: Revalidatie tijdens en na oncologische behandeling: zinvol en haalbaar ? $26^{\mathrm{e}}$ Wenckebach Symposium, Kerkrade. 4 november 2016.

Friesen-Storms J, Lenzen S, Daniels R, Bours G, van Bokhoven M, Jie A, van der Weijden $T$, Beurskens A. Shared decision making about goals and actions in complex care. 14th International Conference on Communication in Healthcare Each Conference, Heidelberg, Germany. 10 Sept 2016.

Friesen-Storms J, Bours G, van der Weijden T, Jie A, Beurskens A. To decide together with cancer patients about aftercare. STTI European Conference, Utrecht. 6 June 2016.

Friesen-Storms J, Bours G, van der Weijden T, Jie A, Beurskens S. Optimaliseren nazorg bij mensen die curatief behandeld zijn voor kanker. Verpleegkundig netwerk Consulenten Maastricht, Heerlen. 16 april 2015.

Friesen-Storms J, Bours G, van der Weijden T, Jie A, Beurskens S. Optimaliseren nazorg bij mensen die curatief behandeld zijn voor kanker. Oncologiecommissie AtriumMC, Heerlen. 10 december 2014.

Friesen-Storms J, Bours G, van der Weijden T, Beurskens S. Optimaliseren nazorg bij mensen die curatief behandeld zijn voor kanker. Benen op de Tafel Sessie. Nazorg bij Oncologie. VGZ, Arnhem. 14 november 2014.

Friesen JHHM, Bours GJJW, van der Weijden T, Beurskens AJHM.Shared Decision Making as a method to underpin the goals of Evidence Based Nursing. Zuyd Hogeschool, Kennis In Bedrijf (KIB), Heerlen. 29 november 2012.

Friesen JHHM,_Bours GJJW, Beurskens AJHM. Workshop met EBP aan de slag. Minisymposium VAR Atrium. 14 november 2012.

Friesen-Storms J, Bours G. Implementatie van EBP in het Atrium Medisch Centrum. Hogeschool Zuyd, Kennis In Bedrijf (KIB), Heerlen. 24 november 2011.

Friesen-Storms J, Bours G, Weijden T, Beurskens S. The implementation of evidence based practice in clinical decision making by nurses. NHG kaderopleiding, Maastricht, September 2011. 
Friesen-Storms J, Moser A, Bours G. Coachingsprogramma EBP afdeling longziekten en kinderafdeling, RAAK project AtriumMC, Heerlen. 2010-2011.

\section{Poster presentations}

Friesen-Storms JHHM, Bours GJJW, van der Weijden T, Beurskens AJHM. Shared decision making in chronic care in the context of evidence based practice in nursing. 1 st International Conference of the German Society of Nursing Science, Berlin, Germany. 5 May 2018.

Friesen-Storms J, Bours G, van der Weijden T, Beurskens A. Shared Decision Making to strengthen Evidence Based Practice in Chronic Care. 14th International Conference on Communication in Healthcare Each Conference, Heidelberg, Germany. 9 Sept 2016.

Friesen-Storms J, Bours G, van der Weijden T, Jie, A, Beurskens A. User-centered development of a conversation approach for shared goal setting and decision making in the aftercare for patients treated for cancer. 7e Wetenschappelijke Symposium. ZuyderlandMC, Heerlen. 23 June 2016.

Friesen-Storms J, Bours G, van der Weijden T, Beurskens A. Shared Decision Making to strengthen Evidence Based Practice in Chronic Care. STTI European Conference, Utrecht. 6-8 June 2016.

Friesen-Storms J,. Bours G, van der Weijden T, Jie A, Beurskens S. Developing and pretesting interventions for Shared Decision Making in the aftercare for patients curatively treated for cancer. Netwerkbijeenkomst Gedeelde Besluitvorming, CBO, Utrecht. 30 juni 2015.

Friesen-Storms JHHM, Bours GJJW, van der Weijden T, Beurskens AJHM. Presented by: Moser A. Shared Decision Making as a method to underpin the goals of Evidence Based Nursing in Chronic Care. ISDM, Peru. 16-19 June 2013.

Friesen J, Bours G, van der Weijden T, Moser A, Beurskens S. The implementation of a Nurse Case Review in a lung unit based on principles of Evidence Based Practice. Annual CAPHRI Research Meeting, Maastricht. 2012. 


\section{Publications in other media}

Verslag in Cruxx. Samen beslissen verbetert zorg in voor- en natraject. 28 maart 2019. https://www.qruxx.com/samen-beslissen-verbetert-zorg-in-voor-en-natraject/

The Breast Cancer Network News for 10 August 2018.

http://myemail.constantcontact.com/BCN-News--Friday--August-10--

2018.html?soid=1103935396778\&aid=XNy5UOSQpZ0

(item 8)

Nieuwsbericht L1 Friesen JHHM Beurs van een ton voor onderzoekster Zuyd. 7 februari 2013. http://www.I1.nl/nieuws/205223-beurs-van-ton-voor-onderzoekster-zuyd

Nieuwsbericht Zuyd Friesen JHHM Jolanda Friesen ontvangt NWO promotiebeurs voor leraren. 7 februari 2013. http://www.zuyd.nl/over-zuyd/nieuws/jolanda-friesenontvangt nwo-promotiebeurs-voor-leraren 


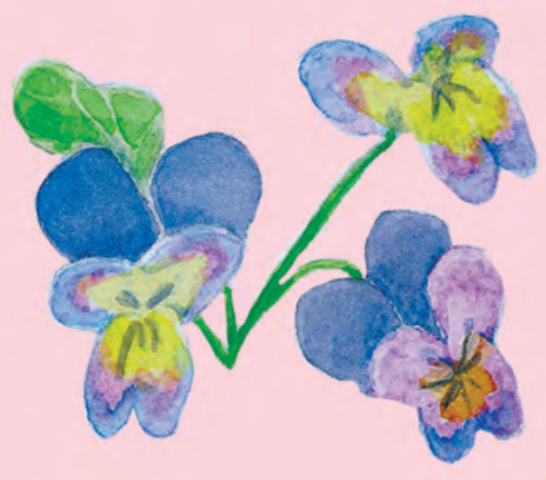

